\title{
Dynamically Stable Legged Locomotion
}

Progress Report: October 1982 - October 1983

\author{
Marc H. Raibert, H. Benjamin Brown, Jr., Michael Chepponis, \\ Eugene Hastings, Jeff Koechling, Karl N. Murphy, \\ Seshashayee S. Murthy, Anthony J. Stentz
}

\section{Leg Laboratory}

The Robotics Institute and Department of Computer Science Carnegie-Mellon University

Pitts burgh, PA. 15213

13 December 1983

This research was sponsored by a grant from the System Development Foundation. and by a contract from the Defense Advanced Research Projects Agency (DoD), Systems Sciences Office, ARPA Order No. 4148. 



\section{Abstract}

This report documents our recent progress in exploring active balance for dynamic legged systems. The purpose of this research is to establish a foundation of knowledge that can lead both to the construction of useful legged vehicles and to a better understanding of legged locomotion as it exists in nature. We have made progress in five areas:

- Balance in 3D can be achieved with a very simple control system. The control system has three separate parts, one that controls forward running velocity, one that controls body attitude, and one that controls hopping height. Experiments with a physical 3D machine that hops on just one leg show that it can hop in place, travel at a specified rate, follow simple paths, and maintain balance when disturbed. Top recorded running speed was $2.2 \mathrm{~m} / \mathrm{sec}(4.8 \mathrm{mph})$. The 3D control algorithms are direct generalizations of those used earlier in 2D, with surprisingly little additional complication.

- Computer simulations of a simple multi-legged system suggest that many of the concepts that are useful in understanding locomotion with one leg can be used to understand locomotion with several legs. A planar model with two legs trots and bounds with the same three part control decomposition used for the one-legged systems. Our most exciting result was to find a particular case for which the model bounds with each leg controlled independently, and without the need for active control of body attitude.

- We have designed a four-legged running machine in order to experiment with balance in systems with more than one leg. The machine is arranged like a large dog, with narrow hips, and a long body. While the leg design for this system is more complicated than our previous designs, the machine is very much like four 3D hopping machines connected by a common frame. Our intention is to study the trot the rack, the gallop and the bound, and a number of gaits that are not normally used by natural quadrupeds.

- We have begun to study gait in terms of coupled oscillations. The question is, "Are gait transitions the result of explicit changes in control, or are they changes in the modal behavior of an oscillating mechanical system?" We do not have an answer yet, but we have made progress in exploring this question with a planar model. We have found that changes in the ratio of leg stiffness to hip stiffness change the pattern of rocking and swaying motions.

- For legged systems to be maneuverable, they must be able to traverse arbitrary paths in the horizontal plane. A useful component in accomplishing this goal is the ability for a legged system to travel a path of arbitrary curvature. We describe simple methods that permit a simulated 3D one-legged system to travel along paths of varying curvature. They depend on jointly manipulating the placement of the foot on each step, and the speed of forward motion.

The report closes with a collection of partially formulated ideas that should stimulate more thinking and lead to further work. 


\section{Table of Contents}

Abstract $\ldots \ldots \ldots \ldots \ldots \ldots \ldots \ldots \ldots \ldots \ldots \ldots \ldots \ldots \ldots \ldots \ldots \ldots \ldots \ldots \ldots \ldots \ldots \ldots \ldots \ldots$

Introduction and summary $\ldots \ldots \ldots \ldots \ldots \ldots \ldots \ldots \ldots \ldots \ldots \ldots \ldots \ldots \ldots \ldots$

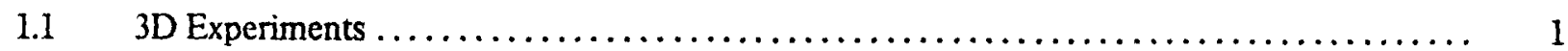

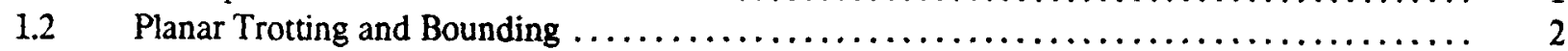

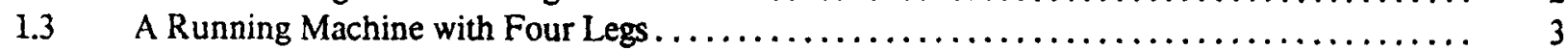

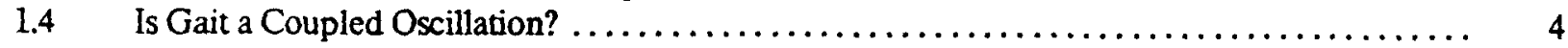

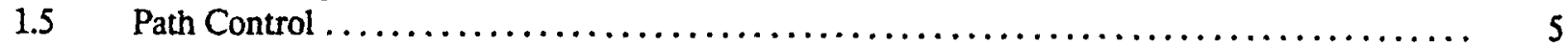

$1.6 \quad$ Legged Locomotion Vignettes $\ldots \ldots \ldots \ldots \ldots \ldots \ldots \ldots \ldots \ldots \ldots \ldots \ldots \ldots \ldots \ldots \ldots \ldots$

2 Experiments with a 3D One-Legged Hopping Machine $\ldots \ldots \ldots \ldots \ldots \ldots \ldots$ Marc H. Raibert, H. Benjamin Brown, Jr., and Michael Chepponis

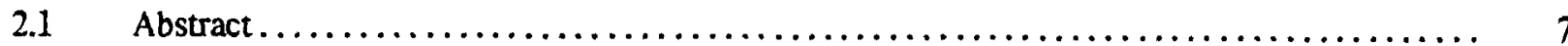

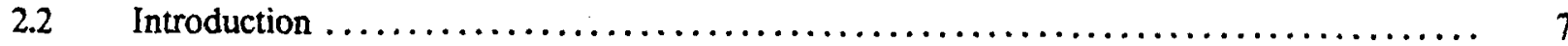

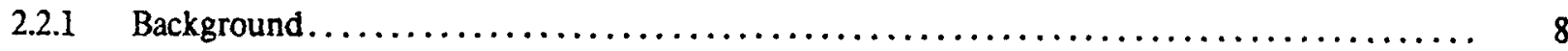

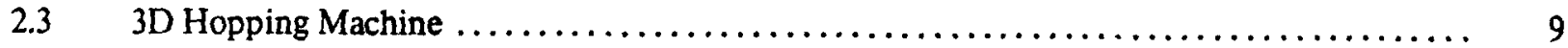

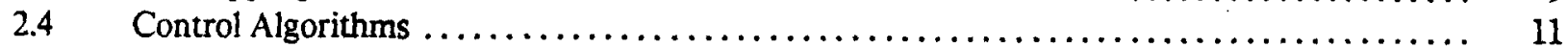

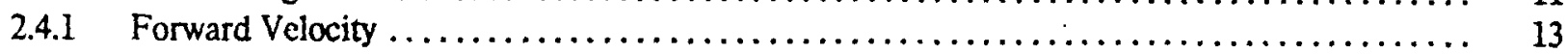

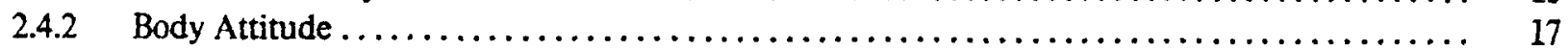

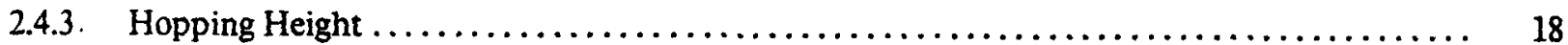

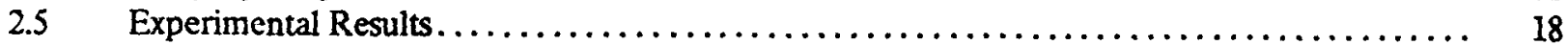

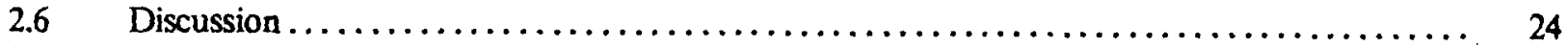

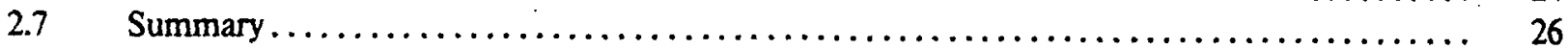

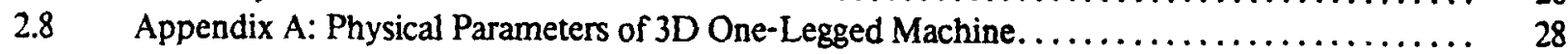

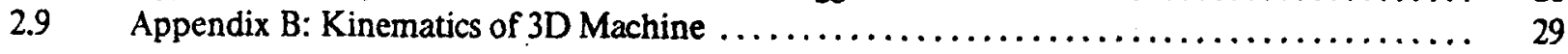

3 Control of Trotting and Bounding for a Simple Planar Model ............. 33 Karl N. Murphy

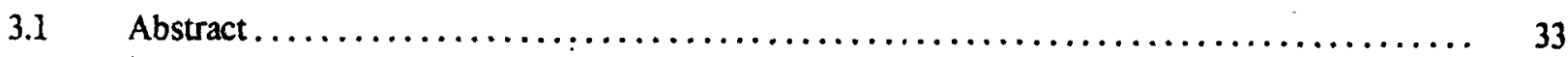

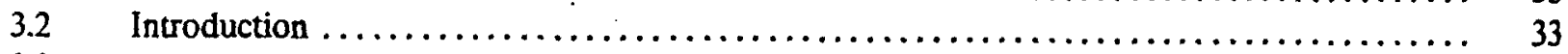

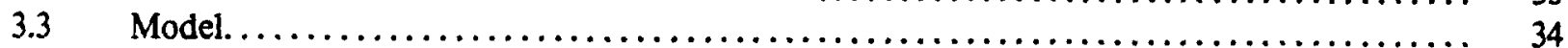

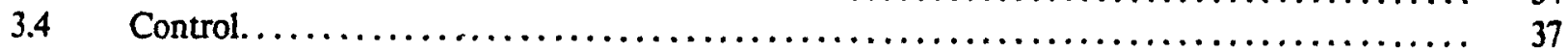

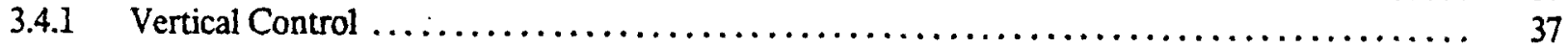

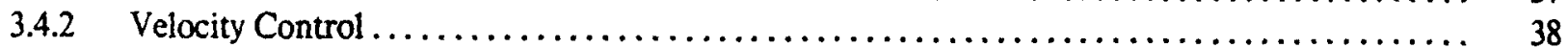

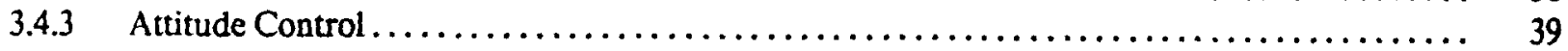

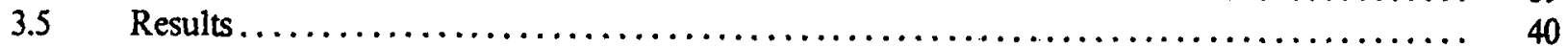

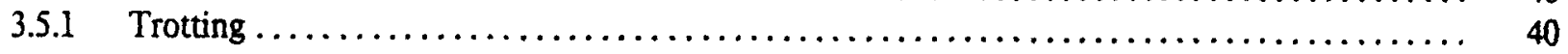

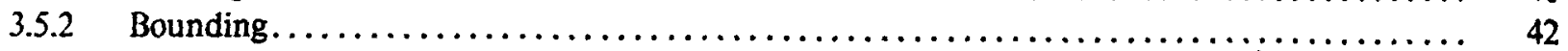

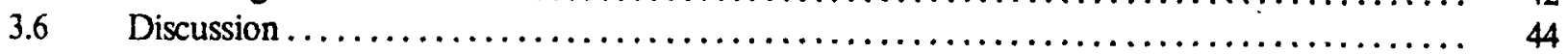

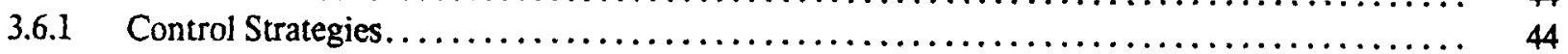

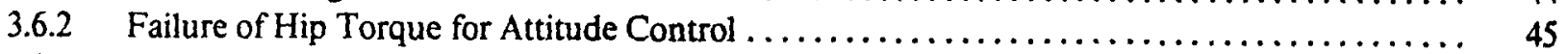

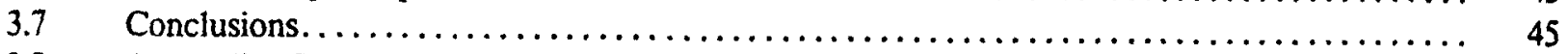

3.8 Appendix. Simulation Parameters. ............................... 47 
H. Benjamin Brown, Jr. and Marc H. Raibert

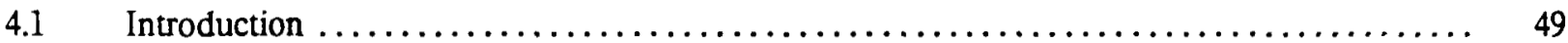

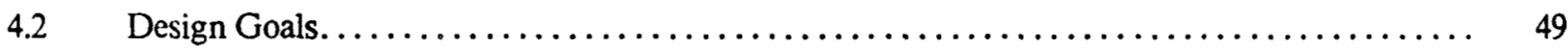

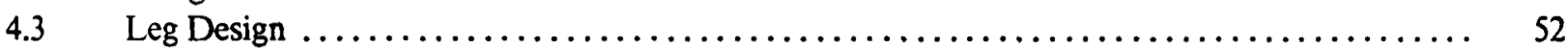

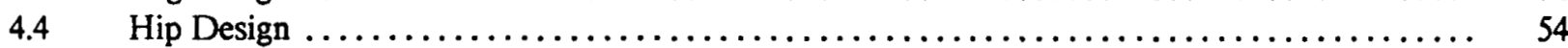

$5 \quad$ Is Gait a Coupled Oscillation?................................. 57

Jeffrey Koechling

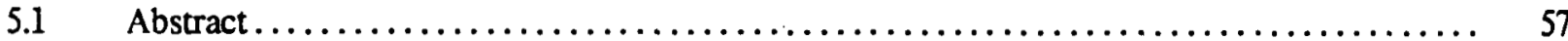

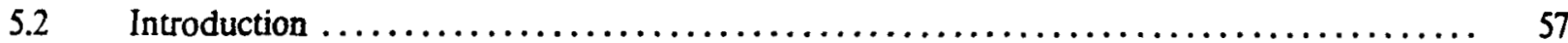

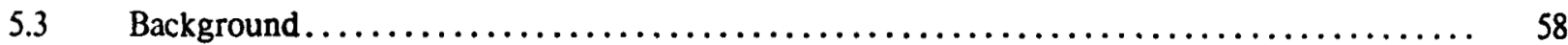

$5.4 \quad$ The Model. ...................................................... 58

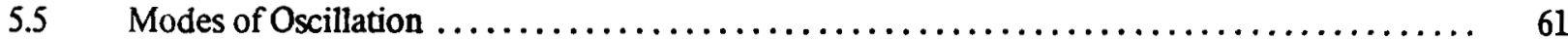

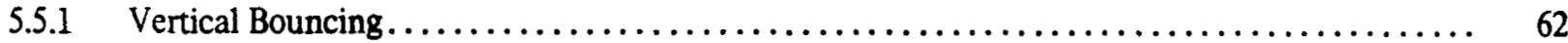

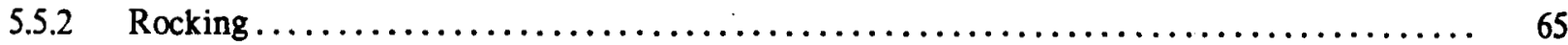

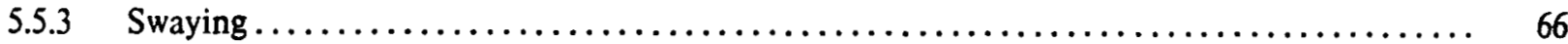

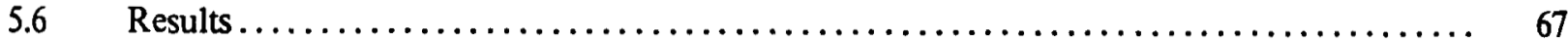

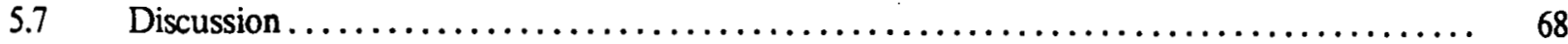

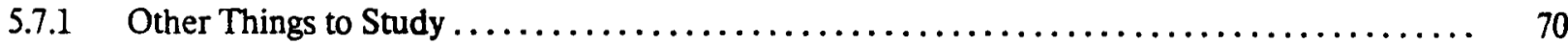

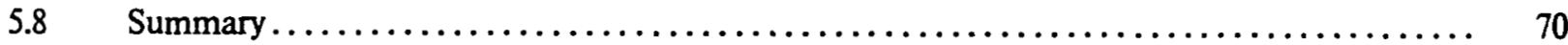

$5.9 \quad$ Appendix A: Equations of Motion for the Model $\ldots \ldots \ldots \ldots \ldots \ldots \ldots \ldots \ldots \ldots \ldots \ldots \ldots \ldots \ldots$

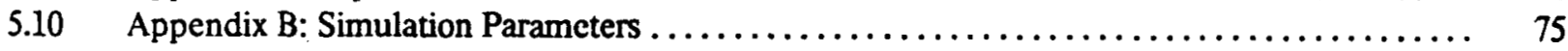

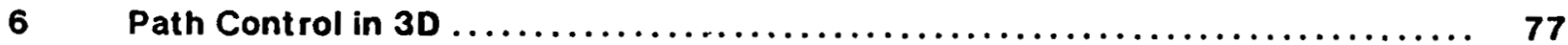

Seshashayee S. Murthy

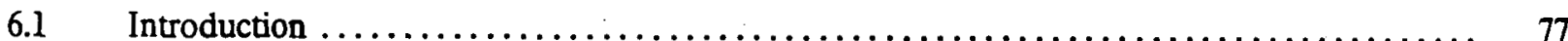

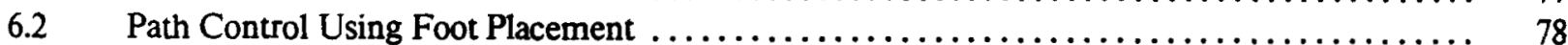

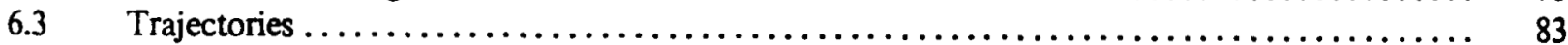

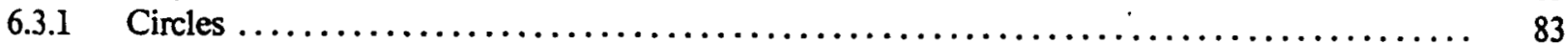

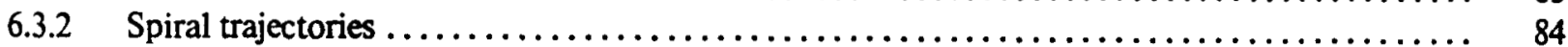

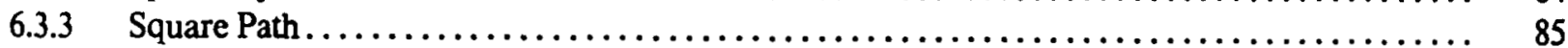

$6.4 \quad$ Appendix: Description of 3D Model ................................... 86

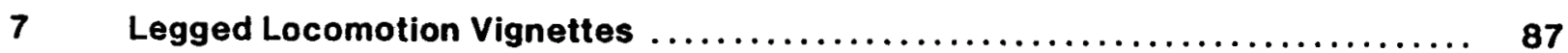

Marc H. Raibert and Anthony J. Stentz

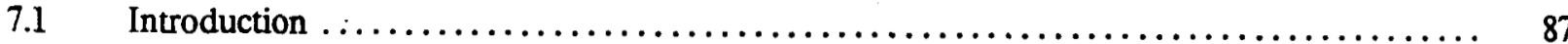

7.2 Locomotion Algorithms for $\mathrm{N}$ Legs. . . . . . . . . . . . . . . . . . . . . . . . . . . . . . . . 87

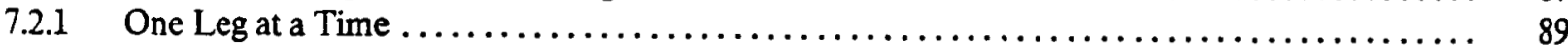

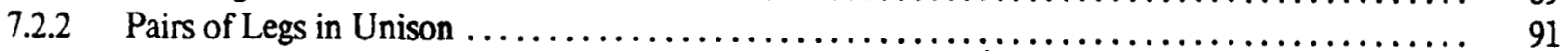

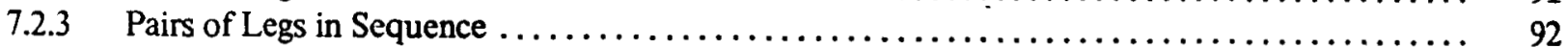

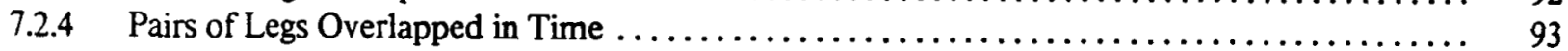

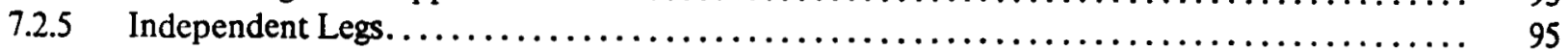

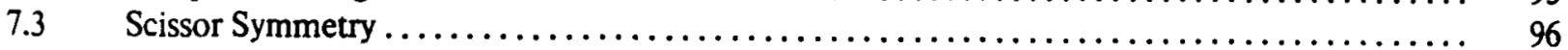

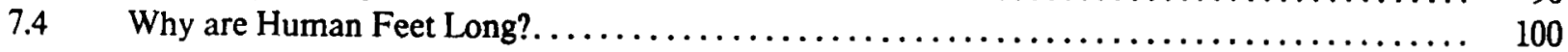




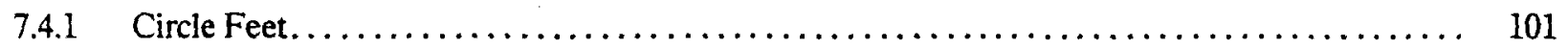

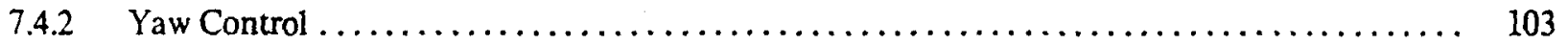

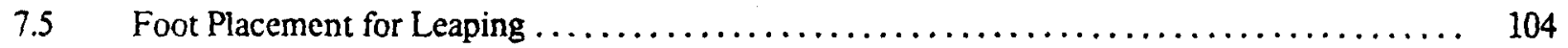

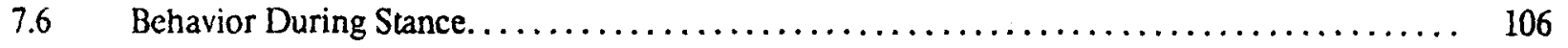

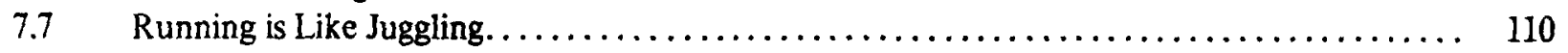

7.8 Do Locomotion and Manipulation Have a Common ground?.................. 112

Bibliography ................................................ 115 


\section{Introduction and Summary}

Humans and animals use their legs to locomote with great mobility, but we do not yet have a full understanding of how they do so. One sign of our ignorance is the lack of man-made vehicles that use legs to obtain high mobility. A legged vehicle might someday travel in difficult terrain, where softness or bumpiness makes wheeled and tracked vehicles ineffective. A component of mobility is the ability to balance. Balance permils a system to move quickly on a narrow base of support, and with intermittent support. Through the research reported here we address both the scientific problem of understanding how living systems achieve balance and control when they run, and the engineering problem of how to build useful legged vehicles.

Our research strategy has been to focus on the problems of balance and dynamic stability, while postponing until later the study of gait and coupling among many legs. To do this we have modeled, simulated, and built a number of systems that hop and balance on just one leg. In the one-legged regime balance is of paramount importance, while coordination and coupling are not important. A secondary strategy has been to examine systems with springy legs, so that we might better understand the role of the resonant bouncing motion that is characteristic of dynamic legged systems.

Our research during the past year has had two main thrusts. One thrust was to extend the results originally obtained for a planar one-legged system to the $3 \mathrm{D}$ case. This was done with computer simulations and physical experiments on a 3D one-legged hopping machine. The other thrust was to explore the problem of locomoting on more than one leg. A simple model that represents the lateral half of a quadruped trots and bounds in simulation, and we have designed a four-legged running machine for experiments. The remainder of this report is a collection of six separate papers that describe these projects. They are summarized here:

\section{$1.13 D$ Experiments}

In order to explore the role of balance in legged locomotion, we are studying systems that hop and run on one springy leg. Previous work showed that relatively simple algorithms can balance a system on one leg when it is constrained mechanically to operate in a plane (Raibert, 1984; Raibert and Brown, 1984). Here we have generalized the approach to a 3D one-legged machine that runs and balances on an open floor without physical support. We decomposed control of the machine into three separate parts: one that controls vertical hopping height, one that controls forward running velocity, and a third that controls attitude of the body. Experiments showed that this control scheme, while surprisingly simple to implement, is powerful enough to permit hopping in place, running at a desired rate, and travel along a simple path. These algorithins that control locomotion in 3D are direct generalizations of those used in 2D, with very little additional complication. 


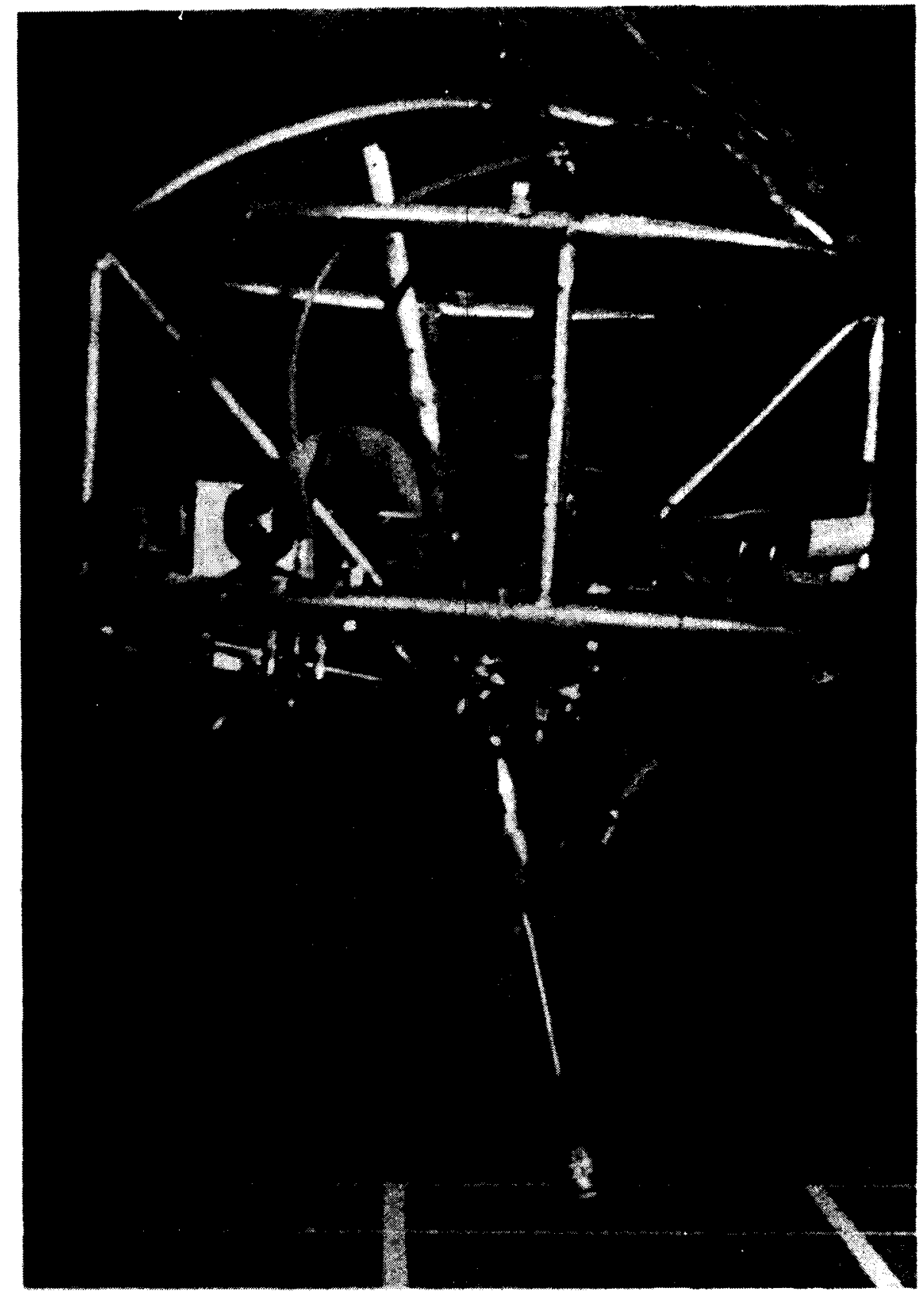

Figure 1-1: Photograph of 3D one-legged machine in mid stride. The machine is running from left to right. Top recorded running speed was about $2.2 \mathrm{~m} / \mathrm{sec}(4.8 \mathrm{mph})$.

\subsection{Planar Trotting and Bounding}

In order to learn about control of locomotion in dynamic systems with more than one leg, we devised a model that looks very much like one lateral half of a quadruped. The model is planar with two springy legs, one attached to the body in the front, and the other attached in the rear. We have found through simulations of this model, that balance during trotting and bounding can be accomplished with mechanisms similar to those used for control of the one-legged systems. One of our most important findings this year is that this model will run with a stable bounding gait, without active stabilization of the body pitch angle. 

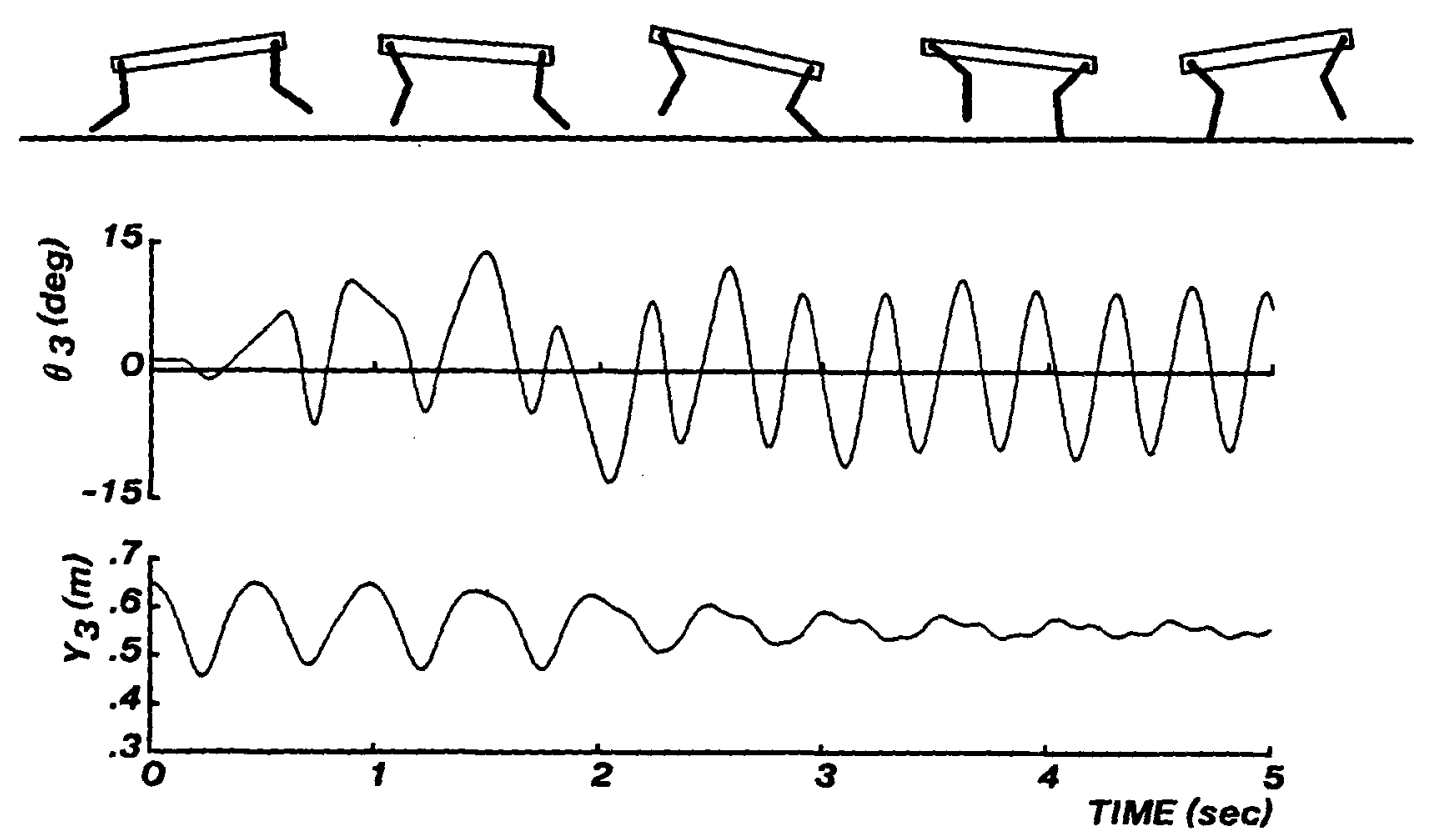

Figure 1-2: Planar two-legged model running with a bounding gait. Each leg of the model is controlled independently to regulate hopping height and forward velocity. The body rocks back and forth in a passively stabilized oscillation, with very litte up and down motion of the center of gravity. When running is initiated there is a random pattern of rocking, but it soon stabilizes. TOP: The cartoon shows behavior when running at about $4 \mathrm{~m} / \mathrm{sec}$. MIDDLE: Atutude of body. BOTTOM: Altitude of body.

During trotting the two legs move in unison and there is very little pitching motion of the body. The control system that generates trotting:

1. Uses the sum of the leg thrusts during stance to regulate desired hopping height.

2. Uses the difference in leg thrusts to control attitude of the body.

3. Uses hip torque during stance to control forward velocity.

4. Uses the position of the feet at touch-down to balance the system.

In a bound the legs act alternately, with each support phase separated by a flight phase. The control system that generates bounding is very similar to that used for trotting, with one exception: no action is taken specifically to control the attitude of the body or its pitching motions. The body pitches back and forth in a passively stabilized motion. While we do not yet fully understand the mechanism responsible for the stability of this oscillation, it seems to hold for a wide range of model parameters and running speeds.

\subsection{A Running Machine with Four Legs}

While experimentation with one-legged systems has taught us a great deal about balance and dynamics in locomotion with a minimum of unnecessary complication, we are eager to extend our experiments to the multi-legged case. The power of the one-legged results will receive the acid test when we attempt to generalize them to the control of machines that run and balance on several legs. 
SIDE VIEW

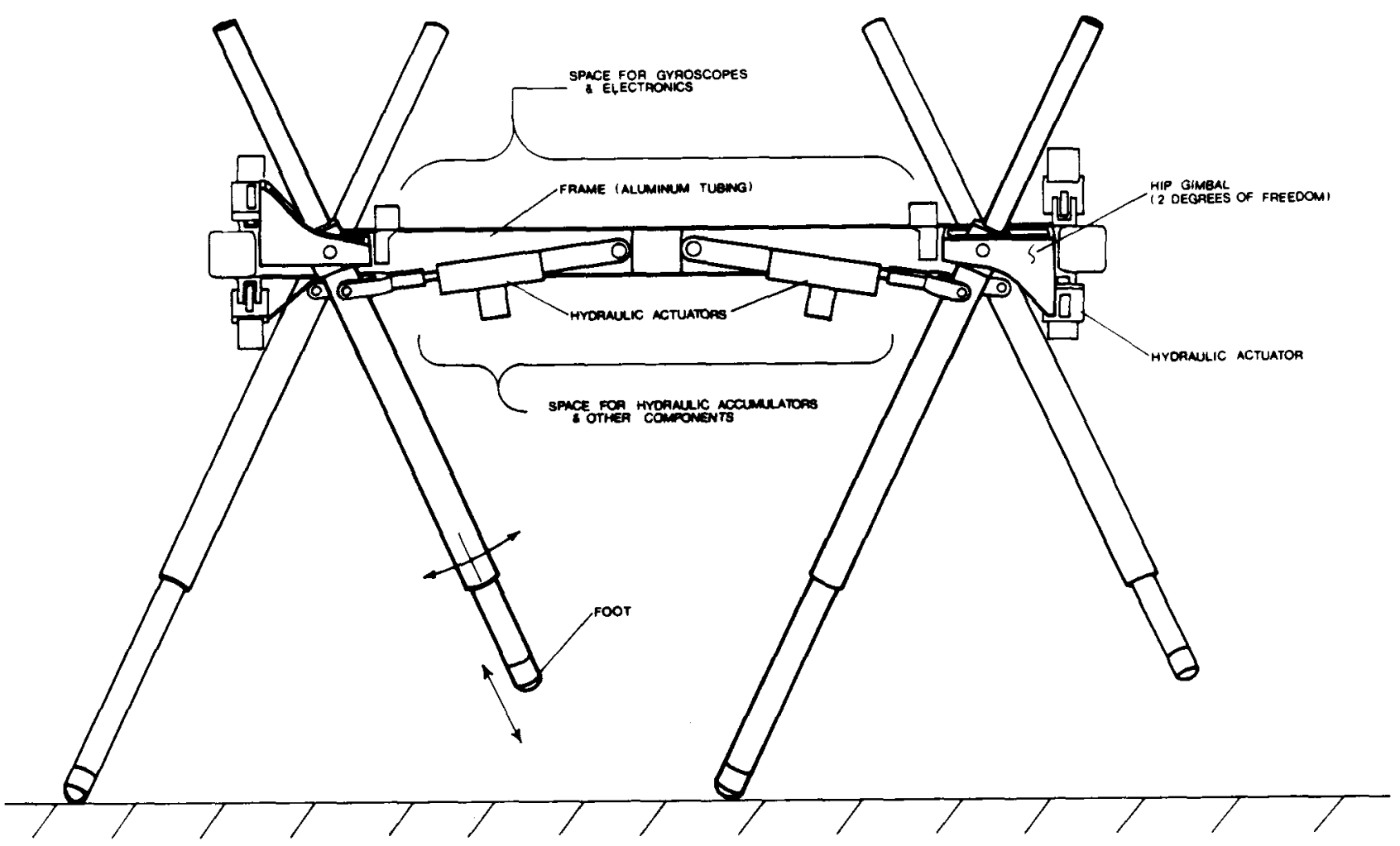

Figure 1-3: Diagram of machine under construction for experiments on balance in multi-legged systems that run. The machine will be about $1.0 \mathrm{~m}$ long. $0.6 \mathrm{~m}$ tall to the hip, and will weigh $35 \mathrm{~kg}$. It is roughly the size and shape of a large dog.

The machine we have designed for these experiments will be very much like four one-legged machines, connected by a common frame. We have imitated the design of quadrupeds found in nature by spacing the hips very close together in the lateral direction, about $0.4 \mathrm{~L}$, and rather far apart in the longitudinal direction, about $1.2 \mathrm{~L}$, where $\mathrm{L}$ is the length of the leg. Although the leg retains use of an air spring to recover hopping energy as did our previous legs, it has been substantially redesigned to meet the additional demands of multi-legged operation. Like our previous designs, the four-legged running machine will carry neither its own power supply nor computing.

\subsection{Is Gait a Coupled Oscillation?}

When animals run at different speeds they use different patterns to coordinate the motion of their legs. These patterns are called gaits. Is gait the fundamental driving pattern for a legged system, or is it merely the observable behavior of a dynamic system constrained by the task of locomotion? This is the question we want to answer.

The idea is that the various gaits we observe in animals could be mechanically coupled oscillations that result when a legged system operates in an efficient manner. The rate of travel, stiffness of the hips, load supported. 


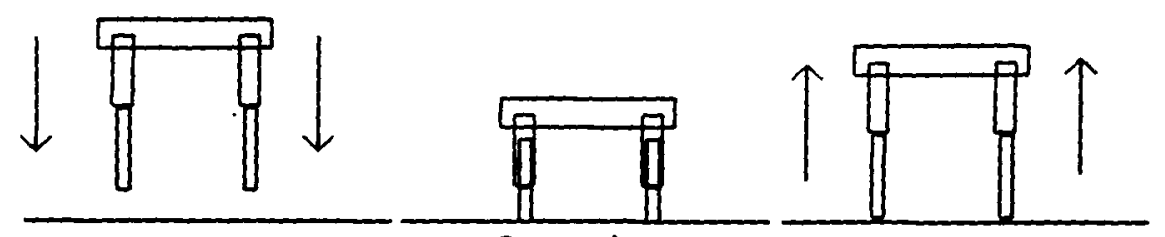

Bouncing
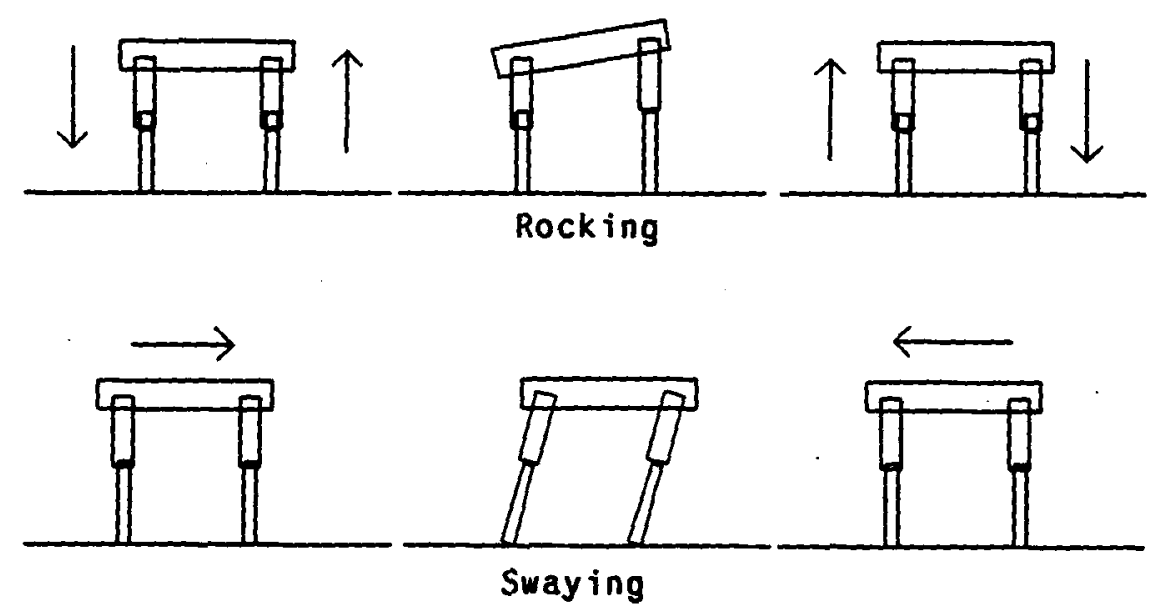

Figure 1-4: Gait as a coupled oscillation. The planar two-legged model has three modes of oscillation, bouncing, rocking, and swaying.

by the legs, and contour of the ground are each factors that should influence the manner in which the legs oscillate, and the timing relationship between their oscillations. At the heart of this idea is the notion that the gait is dominated by the mechanics of the legged system, rather than by the control. Four competing hypotheses guide our thinking:

- The control system determines the pattern and timing of each limb's motion.

- The control system switches from one mode to another when it is efficient to do so.

- The control system adjusts mechanical parameters when increasing speed, with resulting changes in the pattern of oscillation.

- The mechanical system switches oscillation modes as speed changes, with fixed mechanical parameters.

To examine this question we use a planar model that has two springy legs attached to a rigid body. The model has three modes of oscillation: bouncing, rocking, and swaying, as shown in Fig. 1-4. Analysis and computer simulations of the model show that the bouncing and the rocking modes are coupled. The ratio of the stiffness of the hips to that of the legs determines the pattern of rocking and swaying. We have not yet shown that this coupling represents gait-like behavior. 


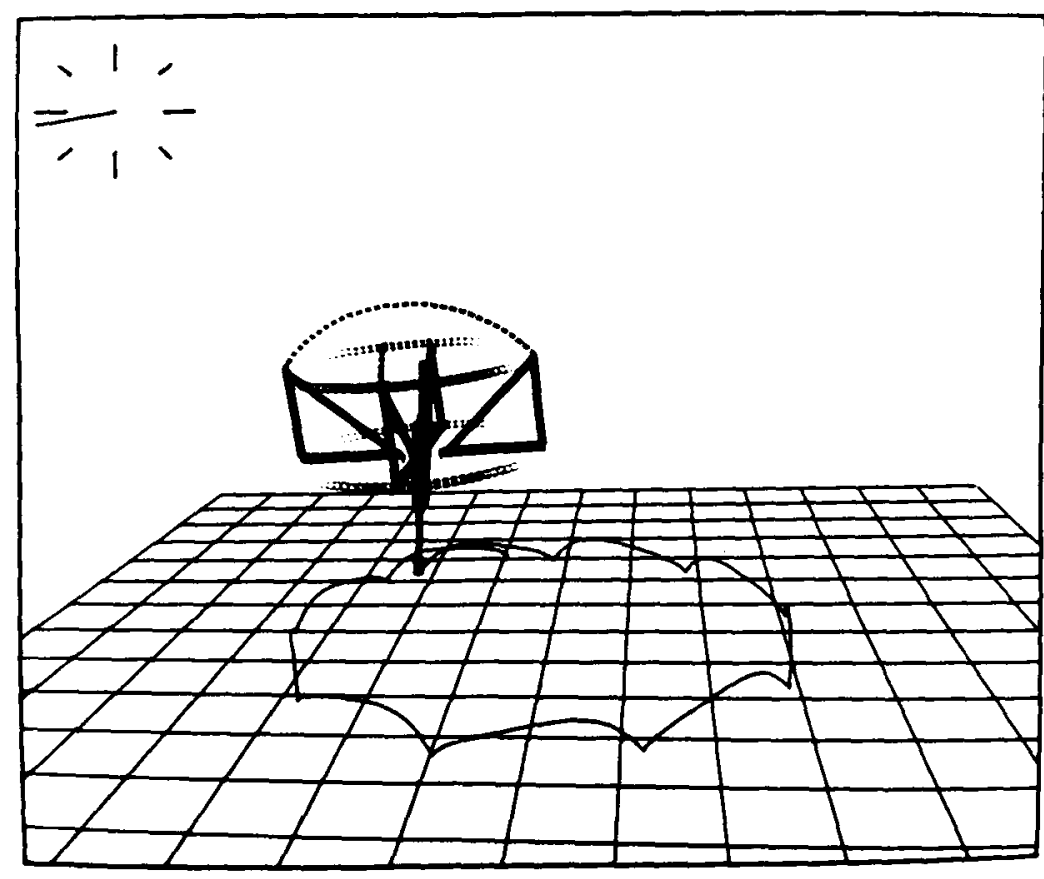

Figure 1-5: Simulated 3D one-legged machine running in circles. A constant lateral ofiset of the foot coupled with constant forward velocity, produces a constant radius of curvature.

\subsection{Path Control}

The ability to traverse an arbitrary path in the horizontal plane will be an important milestone for dynamic legged systems, if they are to achieve maneuverability and to avoid obstacles in their paths. To follow an arbitrary path it is necessary to control both the speed and direction of travel. We have not yet learned how to follow an arbitrary path, but in this section we describe simple methods that permit a simulated 3D onelegged system to generate paths of varying curvature and speed.

\subsection{Legged Locomotion Vignettes}

We have collected together ideas about legged locomotion, that have occurred to us over the past several years. Some of these ideas set the stage for work we plan to do in our laboratory. The sections on Locomotion Algorithms for $N$ Legs and Foot Placement for Leaping fall into this category. Other sections present ideas that are not well formulated, and indeed, may turn out to be wrong. None of the ideas presented in this chapter is backed by experimental data or careful analysis. The purpose of collecting these discussions here is to provide an open repository for our developing thoughts where they can get some air, criticism, and perhaps stimulate better ideas. 


\title{
2. Experiments with a 3D One-Legged Hopping Machine
}

\author{
Marc H. Raibert, H. Benjamin Brown, Jr., and Michael Chepponis
}

\subsection{Abstract}

In order to explore the role of balance in legged locomotion, we are studying systems that hop and run on one springy leg. Previous work has shown that relatively simple algorithms can achieve balance on one leg for the special case of a system that is constrained mechanically to operate in a plane (Raibert, 1984; Raibert and Brown, 1984). Here we generalize the approach to a 3D one-legged machine that runs and balances on an open floor without physical support. We decompose control of the machine into three separate parts: one part that controls forward running velocity, one part that controls attitude of the body, and a third part that controls hopping height. Experiments with a physical 3D one-legged hopping machine showed that this control scheme, while simple to implement, is powerful enough to permit hopping in place, running at a desired rate, and travel along a simple path. These algorithms that control locomotion in $3 \mathrm{D}$ are direct generalizations of those used in $2 \mathrm{D}$, with surprisingly little additional complication.

\subsection{Introduction}

The ability to balance actively is a key ingredient in the mobility observed in natural legged systems, and could be an important factor in man-made legged systems yet to be developed. Actively stabilized legged systems can move on a narrow base of support, permitting travel where obstacles are closely spaced or where the support path is narrow. Systems that balance need not be supported all the time, and may therefore use support points that are widely separated or erratically placed. This ability to place the feet on just those locations that provide good support increases the types of terrain a legged system can negotiate. Biological legged systems routinely operate with narrow base and intermittent support to traverse terrain too difficult for existing wheeled or tracked vehicles.

While the potential advantages of active stability and intermittent support may have been recognized for some time (Manter, 1938; McGhee and Kuhner, 1969; Frank, 1970; Gubina, 1972; Vukobratovic, 1973) progress in building legged systems that employ such principles has been retarded by the perceived difficulty of the task. As a result, much of the previous work on walking machines has taken a quasi-static approach, operating at low velocity with continuous and broad-based support (Frank, 1968; Bessonov and Umnov, 1973; McGhee and Buckett, 1977; Hirose and Umetani, 1980; Sutherland, 1983). These devices have four or six legs, with at least three legs providing support at all times.

Our previous work has shown experimentally that it is possible to control a dynamic legged system that balances actively as it hops and runs (Raibert and Brown, 1984). However, the apparatus of those 
experiments was a planar device, that was constrained mechanically to move with just three degrees of freedom. Useful locomotion takes place in 3 dimensional space, where motion with six degrees of freedom is possible. In this paper we present algorithms that control a legged system that balances as it hops and runs in 3D, and experimental data that characterize the performance. These experiments show that, in the context of a hopping machine with a single springy leg, the control problem need not be difficult at all. A very simple set of algorithms is sufficient to control the machine as it hops in place, as it travels from point to point under velocity or position control, and as it responds to external mechanical disturbances. The control algorithms are direct generalizations of those used in $2 \mathrm{D}$.

\subsubsection{Background}

Previous work on balance began with Cannon's control of inverted pendulums that rode on a small powered truck (Higdon and Cannon, 1963). His experiments included balance of a single pendulum, two pendulums one atop the other, two pendulums side by side, and a long limber pendulum. Their technique was to control the tipping moments by manipulating the point of support with state feedback. Hemami and his co-workers (Golliday and Hemami, 1977; Hemami and Golliday, 1977; Hemami and Farnsworth, 1977; Ceranowicz, 1979; Hemami, 1980), Vukobratovic and his co-workers (Vukobratovic and Stepaneko, 1973; Vukobratovic and Okhotsimskii, 1975), and others (Frank, 1970; Bessonov and Umnov, 1973; Beletskii and Kirsanova, 1976) have studied the dynamic characteristics of a variety of multi-link legged models that walk in simulation. In each case the models balance while maintaining continuous contact with the support surface.

Kato et al. (1981) have studied quasi-dynamic walking in the biped. In their experiments a $40 \mathrm{~kg}$ biped with 10 hydraulically driven degrees of freedom, temporarily destabilizes itself in order to transfer support from one large foot to the other. It uses a pre-recorded sequence of motions to do this. Miura and Shimoyama (1980) have built a number of small electrically powered walking bipeds that balance using tabular control schemes. Their most advanced device, called the stilt biped, walks on two small feet while balancing in 3D. It has three actuated degrees of freedom that permit each leg to move fore and aft, to move sideways, and to lift slightly off the floor. It walks with a pronounced shuffling gait.

Systems with a ballistic phase have also been studied. Seifert (1967) explored the idea of using a large pogostick for transportation on the moon, where low gravity would permit very long hops. He proposed using a moment exchange gyroscope to reorient the body in flight. Matsuoka (1979) analyzed 2D hopping in humans with a one-legged model. He derived a time-optimal state feedback controller that stabilized his model, assuming that the leg could be treated as massless, and that the stance period could be of very short duration. Matsuoka (1980) also implemented a physical planar one-legged hopping machine that operated in a very low-g environment by lying on a table inclined $10^{\circ}$ from the horizontal.

Originally motivated by the conceptual similarity between a pogostick and a leg, Raibert and his co-workers studied planar systems that hop and balance on one springy leg (Raibert, 1984; Raibert and Wimberly, 1984; Raibert and Brown, 1984). They found that for a system constrained to operate in 2D, control could be decomposed into three separate and very simple parts: one to control forward running velocity, one to 
maintain the body in an erect posture, and one to regulate hopping height. These three parts of the control system were each synchronized to the ongoing activity of the hopping machine. This decomposition of the balance problem resulted in a particularly simple control design, and it provided a framework within which. one can think about more complicated problems in locomotion.

In this paper we extend these results for a $2 \mathrm{D}$ system to a system that balances in $3 \mathrm{D}$. The main result is that very simple algorithms are adequate to control the locomotion of a one-legged machine that hops and runs in 3D. The 3D control algorithms are direct extensions of the $2 \mathrm{D}$ algorithms, relying on the same three-part decomposition. The sections that follow describe the physical hopping machine that was used for experiments, they review the $2 \mathrm{D}$ control algorithms and describe their generalization to $3 \mathrm{D}$, and they present experimental data that illustrate the system's ability to balance and run under a variety of conditions.

\subsection{D Hopping Machine}

The hopping machine shown in Fig. 2-1 was designed for experiments on balance in three dimensions. The main parts are a springy leg and a body, connected by a gimbal-type hip. Actuators control the orientation of the leg with respect to the body, and the axial thrust delivered by the leg. Sensors provide state information from the hip, leg, and body to a control computer located nearby in the laboratory.

The body consists of a lightweight platform and roll cage, on which are mounted sensors, valves, actuators, and interface electronics. The ratio of moment of inertia of the body to that of the leg is about $6.5: 1$. This relatively high ratio ensures that movement of the leg during flight does not severely disturb the attitude of the body. The center of mass of the body is located very close to the hip, so the only moments acting on the body are those generated by the hip actuators. A pair of free gyroscopes mounted on the body provide measurements of the roll, pitch, and yaw angles of the body with respect to fixed space. The roll cage protects the hopping machine when it falls over, and also provides convenient handles during experiments.

The leg is a double acting air cylinder. The arrangement of pneumatic cylinder, pressure regulator, and check valve forms an air spring that absorbs energy when the leg shortens under external load, and supplies energy when the leg lengthens. It is storage and recovery of energy in this air spring that transfers the kinetic energy from one hop to the next hop, thereby reducing the cost of continuous hopping. The upper chamber of the pneumatic cylinder that forms the leg actuator, is connected to a pressure regulator that maintains its minimum pressure. This regulator was set to values between 40 and 75 psi for the present set of experiments. A check valve permits the pressure to increase when the leg is compressed, without forcing air back through the system.

The hopping motion is produced by the flow of compressed air to and from the lower chamber of the leg actuator. A pair of 2-way solenoid valves permits this chamber to be pressurized to 80 psi or exhausted. When it is pressurized it causes the piston to move upward and the leg to shorten, and when it is exhausted it causes the piston to move downward and the leg to lengthen. The timing of pressure and exhaust are chosen 


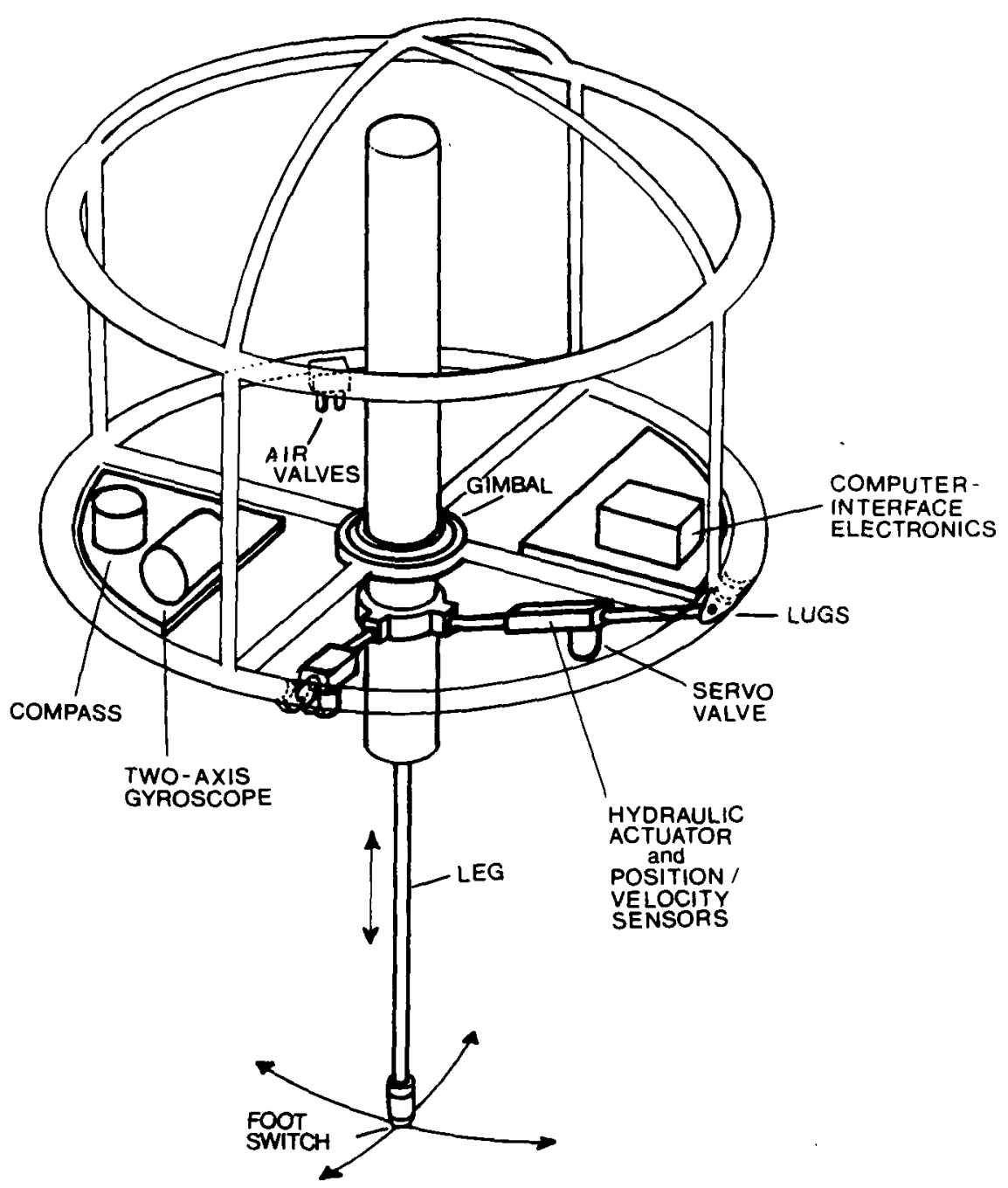

Figure 2-1: Diagram of 3D one-legged machine used for experiments. It has two primary parts: a body and a leg. The body is made of an aluminum frame, on which are mounted hip actuators, valves, gyroscopes, and computer interface electronics. The leg is a pneumatic cylinder with a padded foot at one end, and a linear potentiometer at the other end. Two two-way pneumatic valves control the flow of compressed air to and from the lower end of the leg actuator. A pressure regulator and check valve control the pressure in the upper end of the leg actuator. The leg is springy because air trapped in the leg actuator compresses when the leg shortens. The leg is connected to the body by a gimbal-type hip, with two degrees of freedom. A pair of low friction hydraulic actuators powered by pressure control servo valves acts between the leg and body to determine the hip angles. Sensors measure the length of the leg, the length and velocity of each hydraulic actuator, contact between the foot and the floor, pressures in the leg air cylinder, and the pitch, roll and yaw angles of the body. Analog measurements are digitized on the machine and transmitted to the control computer over a parallel bus. An umbilical cable connects the machine to hydraulic, pneumatic, and electrical power supplies, and to the control computer, ail of which are located nearby in the laboratory

to excite the spring-mass oscillator formed by the leg and body. Peak to peak amplitude of body oscillation under ideal conditions varied between 0.02 and $0.5 \mathrm{~m}$, with corresponding bouncing frequencies of about 3.0 to 1.5 per second. Over this range of bouncing frequencies the stance period is nearly constant, varying by only a few percent, as expected for a spring-mass system. 
A rubber cushion is attached to the lower end of the leg actuator rod to form a foot. The area of the foot that contacts the ground is only about $1 \mathrm{~cm}^{2}$, providing a good approximation to a point support. The coefficient of friction between the foot and the floor in our laboratory is about 0.6. The foot has a built-in switch that tells the control computer when there is contact with the ground. The upper end of the actuator rod carries a wiper that forms the moving element of a linear potentiometer used to mcasure the length of the leg.

The leg and body are connected by a gimbal joint that forms a hip. A pair of linear hydraulic actuators controls the angles between the body and the leg. These hip actuators use only low pressure seals, and a leaky piston to provide very low static friction. Each hip actuator has a pressure control servo valve, a linear potentiometer, and a linear tachometer. The control computer servos the length of these actuators, and thercfore the angles between leg and body, with a pair of linear servos:

$$
\mathrm{f}_{i}(\mathrm{t})=\mathrm{K}_{\mathrm{p}}\left(\mathrm{w}_{i}-\mathrm{w}_{i d}\right)+\mathrm{K}_{\mathrm{v}}\left(\dot{\mathrm{w}}_{i}\right)
$$

where

$$
\begin{aligned}
& \mathrm{f}_{i}(\mathrm{t}) \quad \text { is the force generated by the } i \text { th actuator, } \\
& \mathrm{w}_{i}, \mathrm{w}_{i, d} \mathrm{w}_{i} \text { are the length, the desired length, and velocity of the ith actuator, } \\
& \mathrm{K}_{\mathrm{p}}, \mathrm{K}_{\mathrm{v}} \quad \text { are position and velocity gains. }
\end{aligned}
$$

Using this servo, a full sweep of the leg takes approximately $70 \mathrm{msec}$. This arrangement of body, leg, hip, and actuators provides a means to control the position of the foot and the hip torque needed to balance the system during locomotion.

Data from the sensors mounted on the hopping machine are digitized and transmitted to the control computer over a digital bus. These sensors include the gyroscopes, the hip actuator potentiometers and tachometers, the leg length potentiometer, the foot switch, and the leg pressure sensors. These sensory data are used not only to control the machine, but also to record and analyze its behavior. The umbilical cable that carries the digital communication bus also carries hydraulic power for the hip actuators, compressed air that drives the hopping motion, and DC power for sensors and electronics.

To make the machine balance while traveling from place to place, the control algorithms position the foot during flight and correct the body attitude during stance. During flight the control computer chooses a forward position for the foot appropriate to the machine's rate of travel. During stance the control computer generates torques at the hip to maintain an upright body posture. The resulting control system produces running at rates of up to $2.2 \mathrm{~m} / \mathrm{sec}(4.8 \mathrm{mph})$ with strides of up to $0.79 \mathrm{~m}$. General operation of the machine is shown in Fig. 2-2 by a sequence of photographs taken in one stride.

\subsection{Control Algorithms}

In this section we describe the algorithms examined for hopping and balance in the 3D machine. Since these algorithms were formulated by generalizing from the $2 \mathrm{D}$ machine, we also review the $2 \mathrm{D}$ algorithms. 

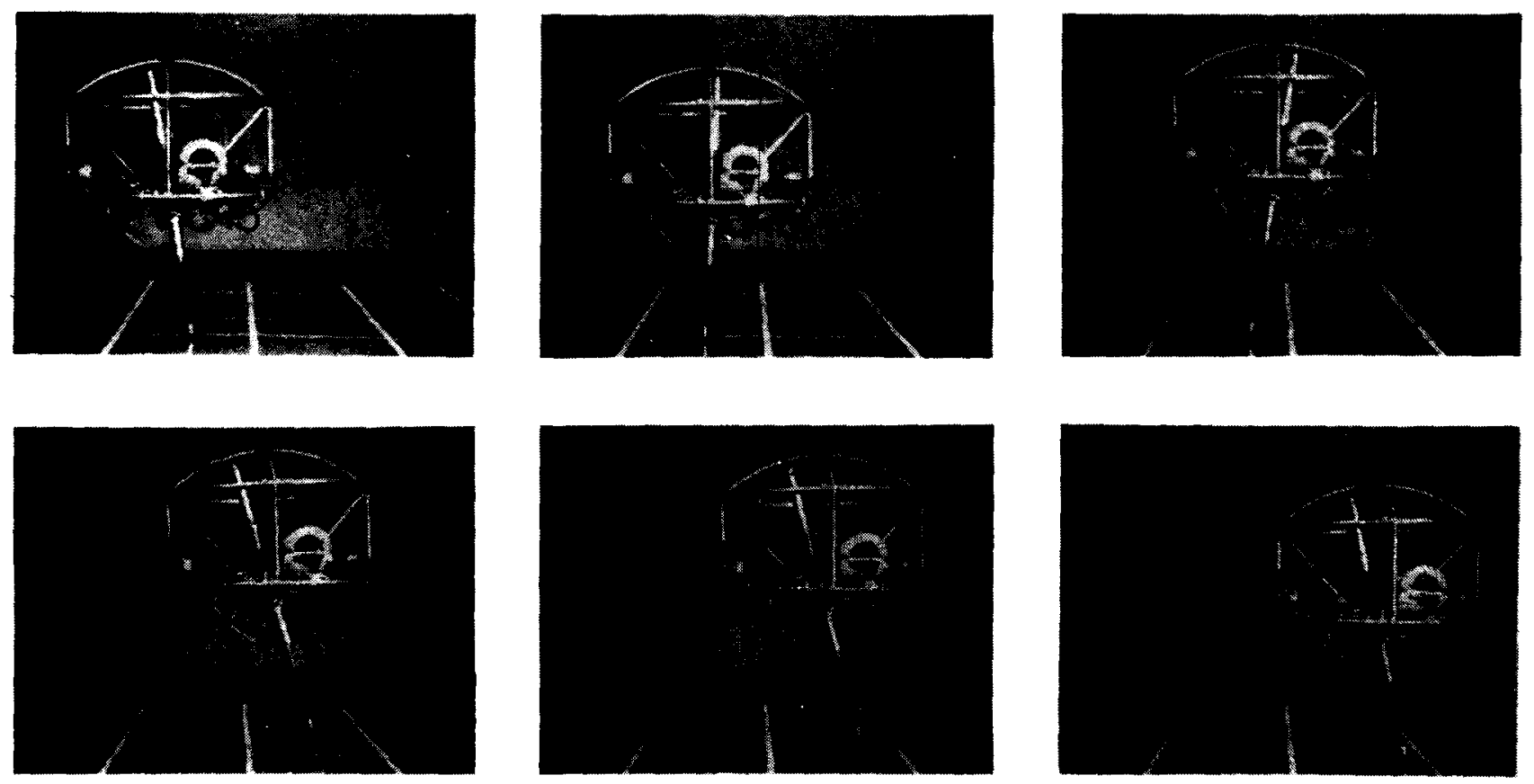

Figure 2-2: Sequence of photographs showing one complete stride of the 3D hopping machine running from left to right. Grid on floor indicates $0.5 \mathrm{~m}$ intervals. Running speed is about $1.75 \mathrm{~m} / \mathrm{sec}$, with stride length $0.63 \mathrm{~m}$, and stride period $0.380 \mathrm{sec}$. Adjacent frames separated by $76 \mathrm{msec}$.

Conceptually, the $2 \mathrm{D}$ and $3 \mathrm{D}$ algorithms are very similar. The basic approach is to treat the system like an inverted pendulum, and to decompose the control into independent parts. As in 2D, the 3D algorithms decompose easily into three parts, one part each for control of forward running velocity, attitude of the body, and hopping height. The system controls forward running velocity by positioning the foot with respect to the projection of the center of gravity. This is done during every flight phase, when the foot is not touching the ground. The system controls the attitude of the body by torquing the hip during stance when the foot is held in place by friction. The system adjusts the hopping height by regulating the amount of thrust delivered by the leg on each hop. These three parts of the control system are largely independent, with their synchronization coming from the ongoing activity of the hopping machine. It is this independence of action that makes the control system simple.

The remainder of this section reviews each part of the control algorithm used in 2D, and describes the corresponding extension to 3D. 


\subsubsection{Forward Velocity}

The position of the foot when it first touches the ground on each step, has a powerful influence on the accelerations the system will experience during the impending support period. The algorithm that controls forward velocity must choose a position for the foot that will generate the proper accelerations. The algorithm studied here uses two factors to find a good position for the foot. One factor is the forward velocity of the system. It is used to find a nominal foot position that would generate zero net acceleration during the support period. The other factor is the forward velocity error. It is used to calculate a displacement of the foot from the nominal position that will accelerate the system as required. Accelerations are required to stabilize the forward velocity against errors, and to generate desired changes in running velocity. The nominal foot position and displacement of the foot combine to specify where the control system will place the foot.

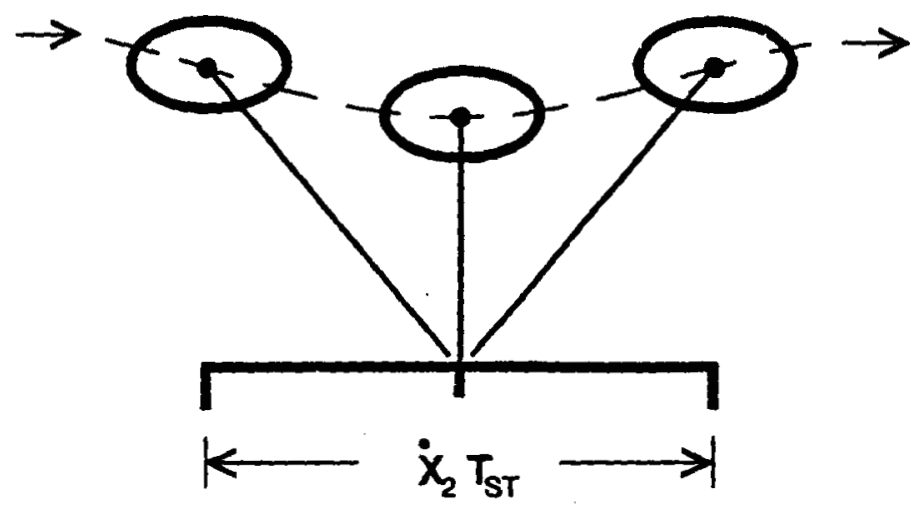

Figure 2-3: When the foot is placed in the center of the CG-print, there is a symmetrical motion. Running from left to right, the left-most drawing shows the configuration just before the foot touches the ground, the center drawing shows the configuration when the leg is maximally compressed, and the right-most drawing shows the configuration just after the foot looses contact with the ground.

The method used to find a nominal foot position that will not accelerate the system depends on producing a symmetrical pattern of motion during the stance phase. During stance, when the foot is touching the ground, the one-legged system is like an inverted pendulum. An inverted pendulum may be kept from tipping over by manipulating the position of the support point with respect to the center of mass in such a way that every tipping motion to one side is compensated by an equal tipping motion to the other side. For a legged system to balance, the control system can position the foot so that there are equal amounts of forward and rearward tipping, and symmetric horizontal forces acting on the ground.

Like the inverted pendulum, a legged system tips and accelerates when its point of support is not located directly below its body. The acceleration magnitude is a function of the horizontal displacement of the support point from the center of mass. A legged system will undergo no net forward accelcration during stance, when the trajectory of the foot with respect to the center of mass is symmetrical about a vertical line 
passing through the center of mass. Figure 2-3 shows such a symmetrical motion for a planar one-legged system. When this symmetry is achieved, the body spends about the same amount of time in front of the foot as it spends behind the foot, so the tipping moments are balanced. The horizontal components of the leg thrust, determined by the leg spring and the angle of the leg to the vertical, are balanced in a similar manner. The forward speed of the system does not change because the horizontal component of the thrust delivered by the leg to the ground averages to zero throughout the stance phase.

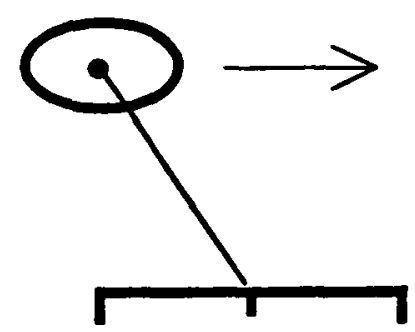

A

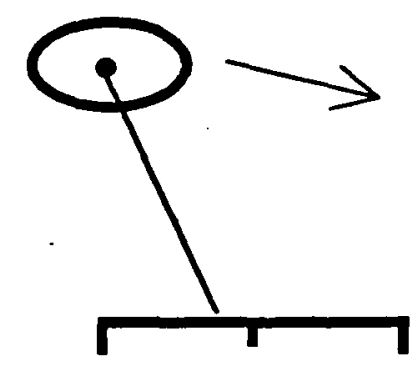

B

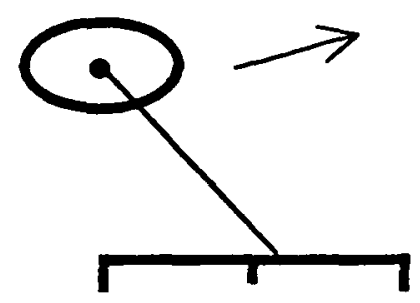

C

Figure 2-4: Behavior when foot is displaced from the center of the CG-print. A: When foot is placed in the center of the CG-print the system tips neither forward nor backward, and it does not change its forward running velocity. B: When foot is placed toward the rear of CG-print, the body tips and accelerates forward during stance. C: When foot is place toward the front of CG-print, the body tips backward and decelerates during stance. Horizontạl lines indicate the CG-print for each case.

In order to achieve symmetry of this sort in the one-legged system, the control algorithm estimates the locus of points over which the center of gravity will travel during the next stance period. We call this locus the CG-print, in analogy to a footprint. The length of the CG-print is the product of the average forward velocity and the duration of stance. The desired symmetry is obiained when the foot is placed in the center of the CG-print.

The control system produces accelerations by placing the foot a distance away from the center of the CGprint. See Fig. 2-4. Placing the foot forward of the center of the CG-print causes the system to spend more time during stance with the body behind the point of support than in front of it. This creates a net backward tipping moment, a net rearward force on the body, and rearward acceleration. Placing the foot behind the center of the CG-print causes the body to spend more time in front of the point of support, creating a net forward acceleration. The algorithm implemented here uses a linear function of velocity errors to calculate displacement of the foot. It uses foot placement to generate these accelerations when the forward velocity deviates from its desired value, or when there is a need to change running speed.

The equations that were used to control forward velocity for the $2 \mathrm{D}$ case are given in terms of the variables defined in Fig. 2-5. Calculate a desired foot position as a function of the forward velocity and the velocity error: 


$$
x_{F, d}=\frac{\dot{x} T_{S I}}{2}+K\left(\dot{x}-\dot{x}_{d}\right)
$$

where

$x_{F, d}$ is the desired forward position for the foot,

$\dot{\mathrm{x}}, \dot{\mathrm{x}}_{\mathrm{d}}$ are the forward and desired forward velocity, and

$T_{S T} \quad$ is the duration of stance.

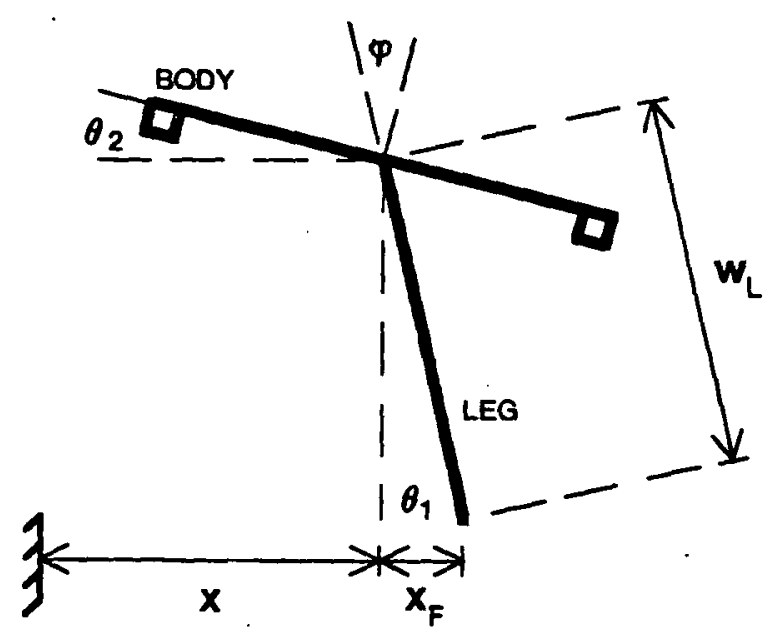

Figure 2-5: Diagram of planar one-legged system that shows variables used in calculating placement of the foot to control forward running velocity.

Once the desired displacement for the foot is known, determine the hip angle that will place the foot there:

$$
\varphi_{\mathrm{d}}=\mathrm{F}\left(\mathrm{x}_{\mathrm{F}, \mathrm{D}}\right)=\theta_{2}+\operatorname{Arcsin}\left[\frac{\mathrm{x}_{\mathrm{Rd}}}{w_{\mathrm{L}}}\right]
$$

To generalize these equations for the 3D case, we modify the forward velocity to include an additional direction of travel. Now the forward velocity has two components, either an $\mathrm{x}$ and $\mathrm{y}$ component as used here, or a magnitude and direction. The kinematics that transform desired foot position into hip angles must also be changed, since body orientation will have three rotations for the $3 \mathrm{D}$ case. Since the forward velocity will now have two components to control, we replace each position and velocity in Eq. (2.2) with a vector:

$$
\mathrm{X}_{\mathrm{F}, \mathrm{d}}=\frac{\dot{\mathrm{X}} \mathrm{T}_{\mathrm{SI}}}{2}+\mathrm{K}\left(\dot{\mathrm{X}}-\dot{\mathrm{X}}_{\mathrm{d}}\right)
$$

$$
\begin{aligned}
\text { where } & \\
\mathbf{X}_{F, d} & =\left[x_{F, d}, y_{F, d}\right]^{T} \text { and } \\
\dot{\mathbf{X}} & =[\dot{x}, \dot{y}]^{T} .
\end{aligned}
$$


The forward velocity, $\dot{\mathbf{X}}$, desired forward velocity, $\dot{\mathrm{X}}_{\mathrm{d}}$, and desired position of the foot with respect to the hip, $X_{F, d}$, are expressed in coordinate systems that do not change orientation in space. Thercfore, the desired motion of the system and measurements of its behavior are expressed in terms of non-rotating coordinates. In practice they are aligned with the walls of our laboratory.

Once the desired position of the foot with respect to the hip is known from Eq. (2.4), find the actuator lengths that will correctly position the foot:

$$
\begin{aligned}
& W_{d}=F\left(X_{F, d}\right) \\
& \text { where } \\
& \qquad W_{d}=\left[w_{1, d}, w_{2, d}\right]^{T} \text {, a vector of desired hip actuator lengths. }
\end{aligned}
$$

$F$ is a function that expresses the kinematic relationship between hip actuator lengths and the foot position. It is an implicit function of leg length, $w_{L}$, and the orientation of the body in space, $\Theta$. F and its inverse, $F^{-1}$, are given in Appendix B. Once $W_{d}$ is known, the linear servo of Eq. (2.1) positions the foot.

Equations (2.4) and (2.5) can be used to control forward velocity once values for the forward velocity, $\mathrm{X}$, desired forward velocity, $\dot{X}_{d}$, and the duration of stance, $T_{s T}$, are known. The foot does not move with respect to the ground during stance, so the forward velocity is the negative of the velocity of the foot with respect to the hip:

$$
\dot{\mathbf{x}}=-\dot{\mathbf{x}}_{\mathbf{F}}
$$

The position of the foot with respect to the hip is given by:

$$
\mathbf{X}_{F}=\mathbf{F}^{-1}(\mathbf{W})
$$

Measurements of $W, w_{L}$, and $\theta$ are available from the actuator sensors and the gyroscopes. We estimate the velocity during stance by numerically differentiating $X_{F}$ as determined from Eq. (2.7). We assume that the forward velocity does not change appreciably during flight. Since the duration of stance, $\mathrm{T}_{\text {sT }}$, is governed by the springiness of the leg, it is largely independent of hopping height, and nearly constant for a given leg stiffness. The control system uses the measured duration of the last stance phase as the expected duration of the next stance phase. The desired forward velocity, $\dot{\mathrm{X}}_{\mathrm{d}}$, is obtained from either a two axis joystick that is manipulated by an operator, or a test program that generates programmable velocity trajectories.

In the current implementation, the center of the CG-print is estimated as $\dot{\mathbf{X T}} \mathrm{ST}_{\mathbf{S T}} / 2$. This estimate is not very good at high velocity or when the duration of stance is very long. Under these circumstances, horizontal forces generated by the leg decelerate the system substantially during the first half of stance, then accelerate it again during the second half of stance. The average forward velocity during stance is less than the forward velocity when the foot first touches the ground, or when it leaves the ground. Therefore, the length of the CG-print is substantially shorter than estimated. While we are working on better methods for estimating the CG-print, (Raibert et al., 1983b), this problem is not too important in practice. The estimation error results in a velocity dependent, steady state error in forward velocity that increases at high rates of travel. 


\subsubsection{Body Attitude}

Since angular momentum of legged systems is conserved during flight, a control system can manipulate the body attitude only during stance, when there is traction between the foot and the ground. Torques generated between the leg and the body during stance are used to servo the attitude of the body to a desired orientation. For the planar case the stance servo was:

$$
\begin{aligned}
& \tau(\mathrm{t})=\mathrm{K}_{\mathrm{p}}\left(\theta_{2}-\theta_{2 \mathrm{~d}}\right)+\mathrm{K}_{\mathrm{v}}\left(\dot{\theta}_{2}\right) \\
& \text { where } \\
& \tau \text { is the hip torque, } \\
& \mathrm{K}_{\mathrm{p}}, \mathrm{K}_{\mathrm{v}} \text { are position and velocity gains, and } \\
& \theta_{2, d} \text { is the desired attitude of the body. }
\end{aligned}
$$

For the 3D system, both the pitch and roll axes must be controlled during stance:

$$
\begin{aligned}
& f_{1}=K_{P}\left(\theta_{P}-\theta_{P, d}\right)+K_{V}\left(\dot{\theta}_{P}\right) \\
& f_{2}=K_{P}\left(\theta_{R}-\theta_{R, d}\right)+K_{v}\left(\dot{\theta}_{R}\right) \\
& \text { where } \\
& \quad f_{1}, f_{2} \quad \text { are the hip actuator forces, } \\
& \quad K_{P,}, K_{v} \quad \text { are position and velocity feedback gains, and } \\
& \theta_{\mathrm{P}, \mathrm{d}}, \theta_{R, d} \text { are the desired pitch and roll angles, zero in this paper. }
\end{aligned}
$$

The pitch and roll angles upon which these attitude control servos operate are defined in a coordinate system that moves and rotates with the body. They are not corrected for rotations of the body about its yaw axis, as the forward position and velocities are. The gyroscope is aligned so that the signal from one axis can be used to servo one hip actuator, and the signal from the other axis can be used to servo the other hip actuator. This simple arrangement requires very little computation and provides very good stability.

In addition to keeping the body erect, the control system is responsible for controlling the facing direction of the body, the yaw angle. This is a degree of freedom that has no counter-part in 2D. In principle, it is possible to generate torques about the yaw axis for this purpose, despite the lack of an actuator that twists the foot about the leg axis. When the control system places the foot to one side of the direction of travel and torques fore or aft at the hip during stance, a moment is developed about the yaw axis of the system. In order to stabilize the system during such a maneuver, the foot can be offset in one direction on one hop, and in the other direction on the next hop.

We have found through experimentation and subsequent analysis that the maximum yaw torque that can be generated in this manner is substantially smaller than the disturbance torque generated by the umbilical cable that connects the machine to power supplies and computer. Therefore, the control system was not able to generate adequate yaw torque to control the facing direction of the 3D hopping machine. Instead, the control system used measurements of the yaw angle to compensate for the facing direction of the machine, without trying to control it. 


\subsubsection{Hopping Height}

For a legged system to locomote each leg must alternate between a support phase, in which the foot touches the ground and bears weight, and a transfer phase when the foot is elevated to move from one foothold to another. An alternation of this kind between loaded phases and unloaded phases, underlies the normal activity of all sorts of legs in all sorts of legged systems. For a system with one leg, this alternation is the hopping cycle.

Unlike control of forward velocity and body attitude, control of hopping height is no different in $3 \mathrm{D}$ than it was in 2D. Hopping is accomplished by exciting the resonant spring-mass system formed by the leg and body. In principle, the height of each hop will be determined by the kinetic and potential energies of the system, and the losses encountered on each bounce. Manipulation of these energies could be used to control the height to which the system hops (Raibert, 1984). A simpler technique was used in practice.

If the system were left to bounce passively on the springy leg, losses in the sliding friction of the air cylinder and in accelerating and decelerating the unsprung mass of the leg would soon cause the machine to come to rest. Measurements of the decay in hopping height during passive bouncing showed that such energy losses amounted to about a $35 \%$ loss on each bounce. The leg actuator delivers a vertical thrust on each cycle that just compensates for these losses.

Hopping height is regulated by providing a fixed thrust on each hop. Equilibrium occurs when the energy lost in one hopping cycle equals the energy introduced through the leg actuator. Since losses are monotonic with hopping height, a unique hopping height exists for each value of leg actuator thrust. Details of the relationship between hopping height and duration of thrust can be determined empirically.

\subsection{Experimental Results}

The one-legged machine described earlier was used to evaluate and refine the control algorithms, and to demonstrate balance in a 3D running machine. The height. velocity, and attitude control algorithms of the last section were implemented in a set of control programs that ran on a control computer. These programs controlled the machine and recorded its behavior. The experiments tested velocity control, position control, the ability to follow a simple path, and the hopping machine's resistance to disturbances.

We examined the system's ability to regulate forward running velocity by having the control computer specify a ramp in desired velocity. The results are plotted in Fig. 2-6. These data show the machine, first hopping in place, then running at increasing rates up to about $1.7 \mathrm{~m} / \mathrm{sec}$. Throughout the run velocity was controlled to within about $0.2 \mathrm{~m} / \mathrm{sec}$ of the desired value. This accuracy is typical. When the desired velocity was set to zero at $\mathrm{t}=5.3 \mathrm{sec}$, it took about $0.5 \mathrm{sec}$ for the velocity to change. This was the delay between the change in $\dot{x}_{d}$ and the following touchdown.

During running, the leg and body counter-oscillate as shown in the plots of $\mathrm{X}_{\mathrm{F}}, \boldsymbol{\theta}_{1}$ and $\boldsymbol{\theta}_{2}$. The back and forth 

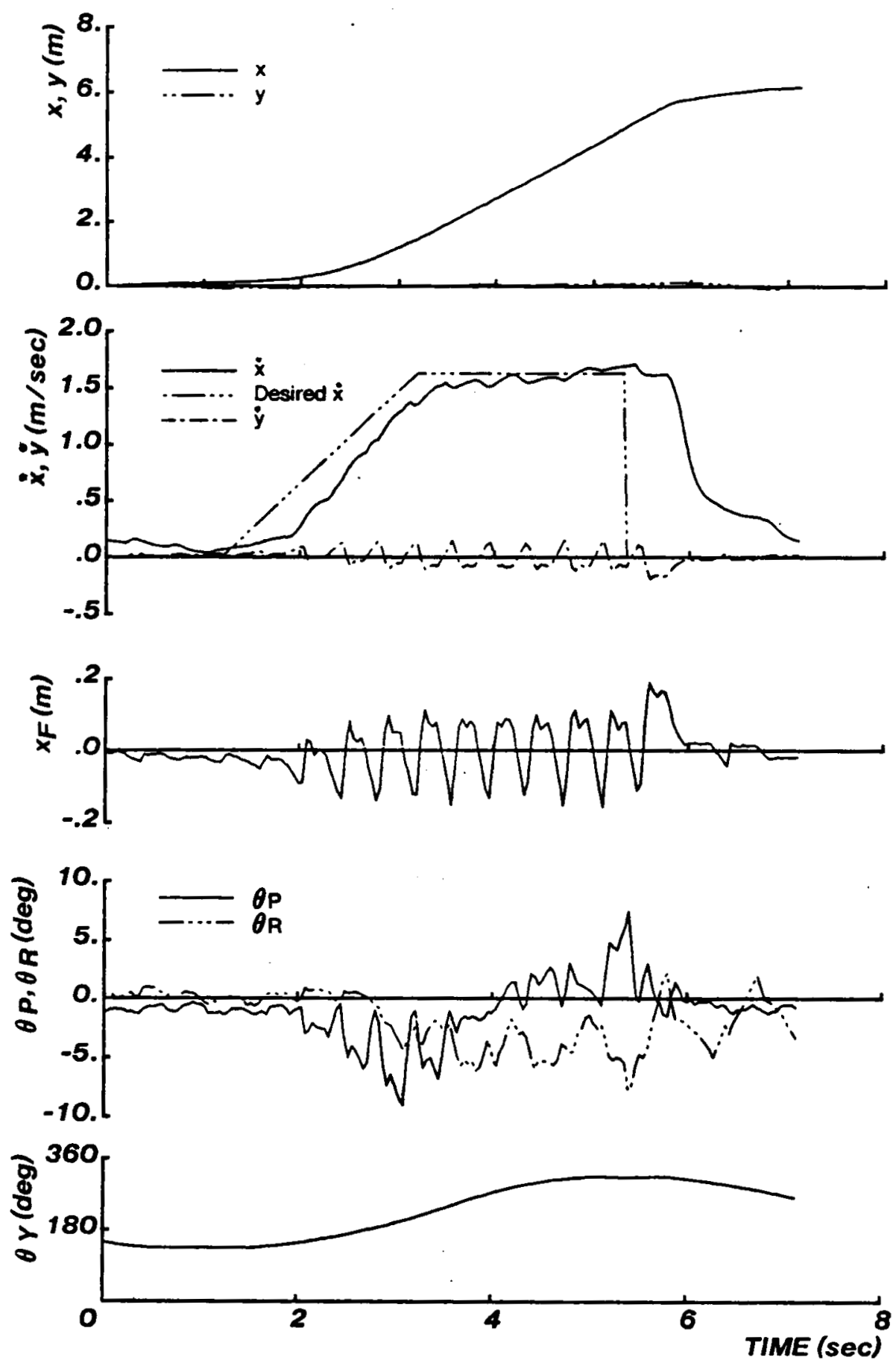

Figure 2-6: Velocity control was examined by varying the desired velocity in the $\mathrm{x}$ direction from 0 . to $1.6 \mathrm{~m} / \mathrm{sec}$ with an acceleration of $1 \mathrm{~m} / \mathrm{sec}^{2}$, then holding the setpoint constant for about 2 seconds, and then setting the rate setpoint to zero. (Dashed line in second plot.) Facing direction of the body, $\theta_{Y}$, was measured but not controlled Also shown are TOP: the position of the machine in the room, MIDDLE: the position of the foot with respect to the hip, BOTTOM 2: and the yaw orientation of the body. (3D.335.12) 

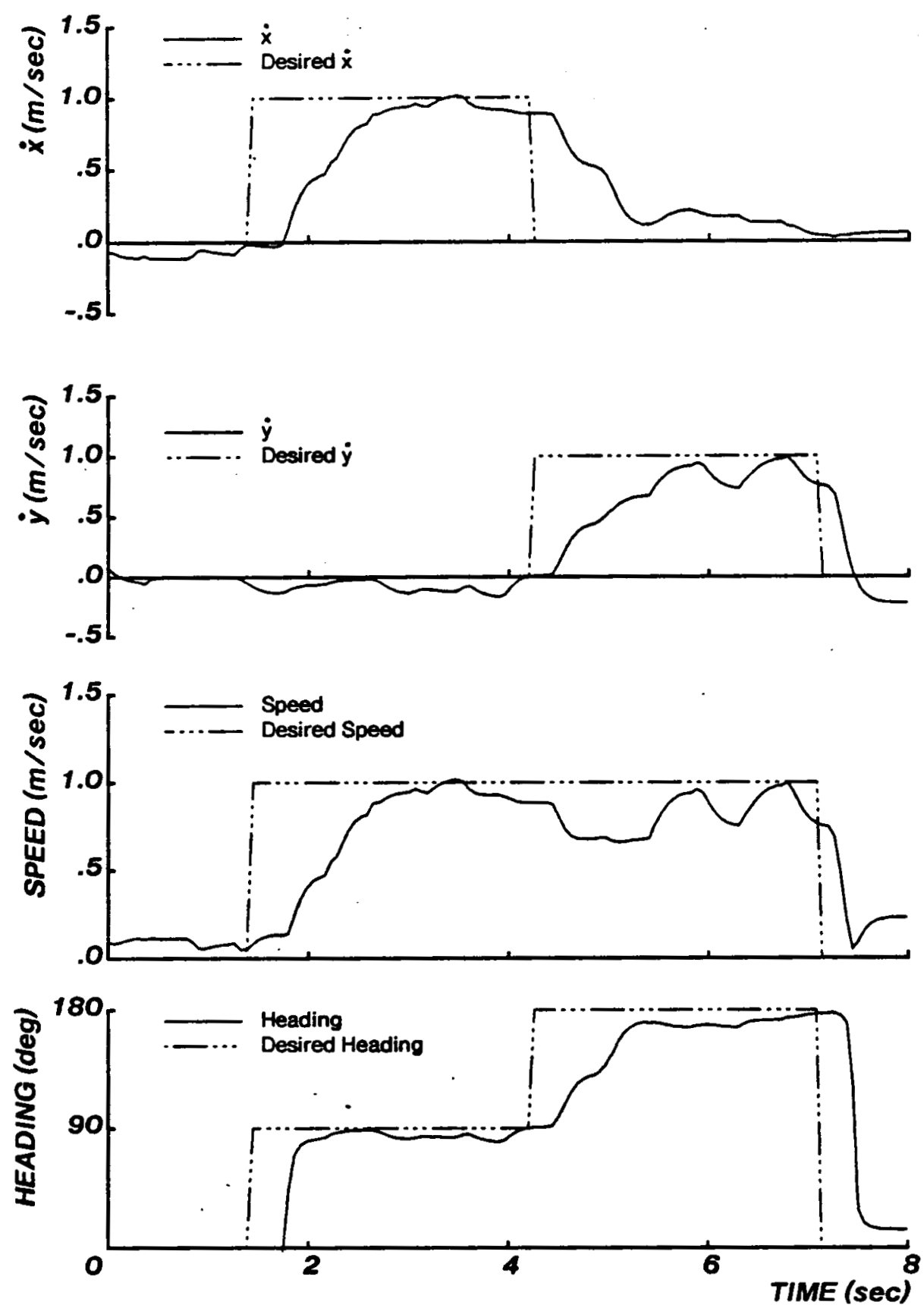

Figure 2-7: A step change in desired direction was programmed to generate a right-angle turn, while holding desired speed constant. The top two curves plot the $x$ and $y$ velocities, and the bottom two show the speed and heading. The turn was completed in two steps. (3D.335.5) 
motions of the leg were not explicitly programmed, but resulted from interactions between the velocity controller that positioned the leg forward during flight, and the attitude controller that operated during stance. Oscillations of the body were expected, because angular momentum is conserved during flight, and attitude correction occurs only during stance. The asymmetry in body attitude was also expected, since the desired body angle, $\theta_{d}$, was always zero. The relative magnitudes of the pitch and roll oscillations varied as the facing direction of the machine, its yaw angle, changed.

In another experiment, the desired speed was held constant, but the desired direction was changed abruptly by $90^{\circ}$. The results are shown in Fig. 2-7. It took two hops for the system to change direction, but speed was erratic after the turn.

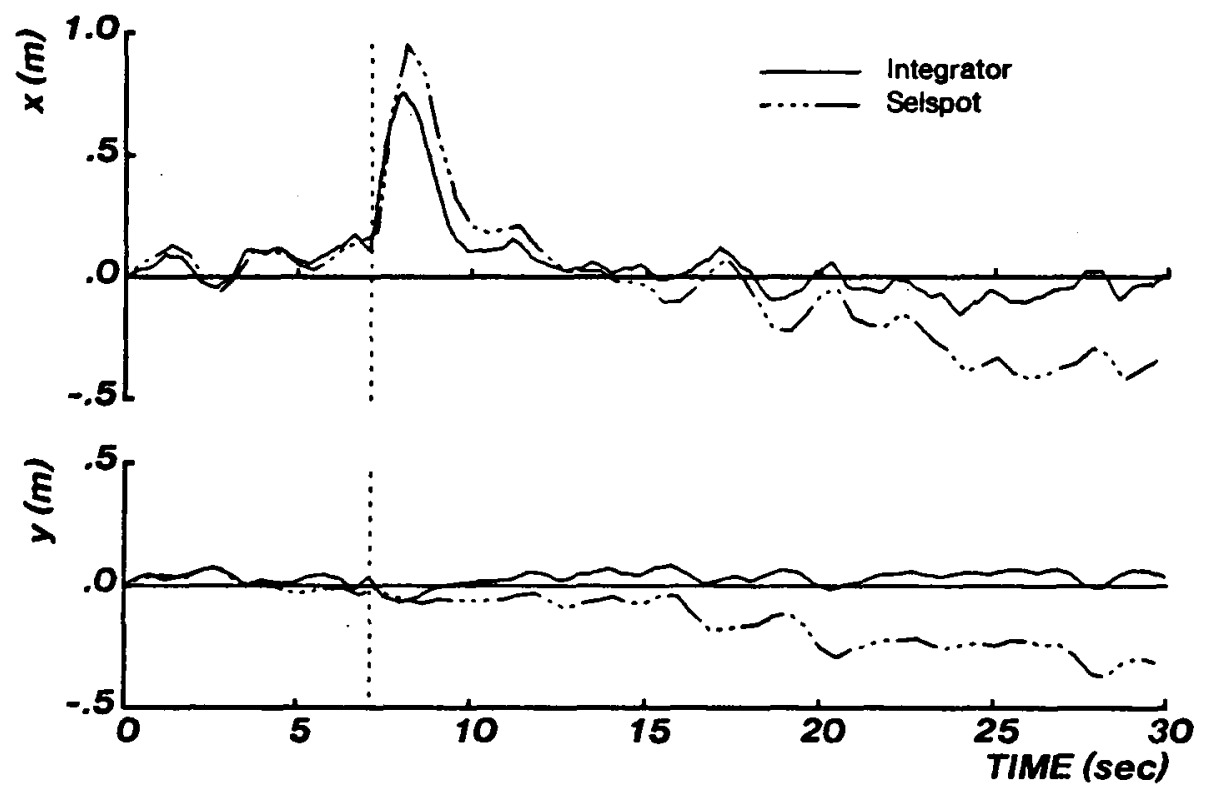

Figure 2-8: 3D machine hopping in place under position control. The control system integrated fonvard velocity to determine the machine's position in the room. An electro-optical system (Selspot) mounted on the ceiling provided an independent measurement of the machine's position. Divergence between Selspot and integrator data indicates drift in the integrator. DOTTED LINE: Experimenter disturbed the machine by delivering a sharp horizontal jab to the frame with his hand It returned to the position setpoint within a few seconds. (3D.332.4)

A position control algorithm was used to make the hopping machine hop in one place, and to translate from place to place. The position control algorithm transforms position errors into desired velocities: 


$$
\begin{aligned}
& \dot{\mathrm{X}}_{\mathrm{d}}=\mathrm{K}_{\mathrm{p}}\left(\mathrm{X}-\mathrm{X}_{\mathrm{d}}\right)+\mathrm{K}_{\mathrm{v}} \dot{\mathrm{X}} \\
& \dot{\mathrm{X}}_{\mathrm{d}}=\min \left\{\dot{\mathrm{X}}_{\mathrm{d}}, \dot{\mathrm{X}}_{\mathrm{d} \text { max }}\right\} \\
& \text { where } \\
& \quad \mathbf{K}_{\mathrm{p}}, \mathrm{K}_{\mathrm{p}} \quad \text { are diagonal position and velocity gain matrices, and } \\
& \dot{\mathrm{X}}_{\mathrm{d} \text { max }} \text { is a limit on the allowable velocity. }
\end{aligned}
$$

The control programs obtained information about the machine's position in the room in two ways. They could estimate the position of the machine by numerically integrating the forward velocity estimate, $\dot{\mathbf{X}}$. Position information was also available from an electro-optical sensor (Selspot) mounted on the ceiling of the laboratory. Data could be read from this sensor by the control computer one time per hop in order to calculate a new desired velocity using Eq. (2.10). One may think of the ceiling mounted sensor as serving the same role as the geosynchronous satellites used for global navigation. Our geosynchronous satellite had a very low orbit.

Figure 2-8 is a plot of the machine's position as it hopped in place, using the integrator position values for control. The machine stayed within $0.25 \mathrm{~m}$ of the setpoint. Deviations of this magnitude were typical for stationary hopping. In addition to integrator data, data are plotted from the electro-optical measurement. The deviations in these curves shows that the integrator drifts by about $0.005 \mathrm{~m} / \mathrm{hop}$. This means that if the machine were instructed to hop in one spot, it might drift a meter in one minute. Informal experiments with blindfolded humans hopping on one leg indicate that they drift by similar amounts. In the case of the hopping machine, the primary sources of drift were gyroscope calibration errors, and unwanted forces exerted on the machine by the umbilical.

Figure 2-8 also shows the response to an external disturbance. After about 7 seconds the experimenter delivered a sharp horizontal jab to the body as the machine hopped in place. (See dotted vertical line in Fig. 2-8.) The machine maintained its balance and returned to the position setpoint after a few seconds. The control system tolerated fairly strong disturbances of this sort. The system also tolerated substantial torsional disturbances of this sort, as well as moderate roll and pitch disturbances.

In order to measure performance under position control, the control computer specified desired positions according to a preplanned sequence. An operator pressed a button every time he wanted the next position setpoint from the sequence. In this way we programmed a square path, $2 \mathrm{~m}$ on a side. Figure $2-9$ plots data obtained while traversing such a path, and Fig. 2-10 is a photograph of the machine traversing a square path. The data shown in Fig. 2-9 are pretty good, with the exception of a fixed position error of about $0.3 \mathrm{~m}$ when $y_{d}=0$. This error was caused by the umbilical cable, which was just long enough to permit the machine to reach $y=0$. The system came to equilibrium where the force exerted by the umbilical cable equalled the accelerations produced by the control system. 

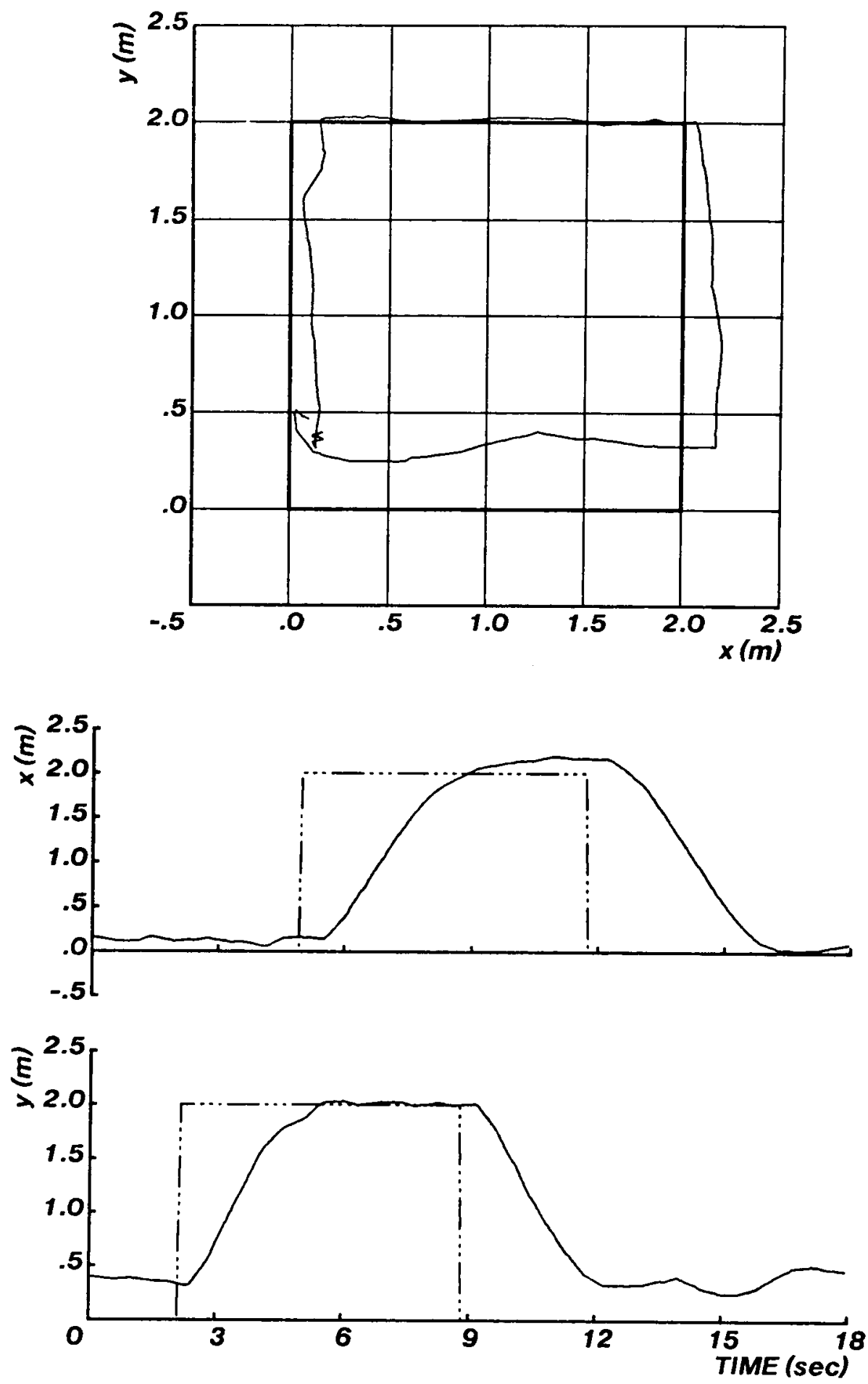

Figure 2-9: Data recorded while 3D hopping machine traversed a square path. The control system integrated forward velocity estimates to deternine the position of the machine. TOP: Desired and measured path of machine plotted in $X-Y$ plane. BOTTOM: Plots of $X$ and $Y$ position as a function of time. The data plotted are the recorded integrator values. The desired path is shown bold in the top plot. It extends from $(0,0)$ through $(0,2),(2,2),(2,0)$, and $(0,0)$. (3D.332.5) 


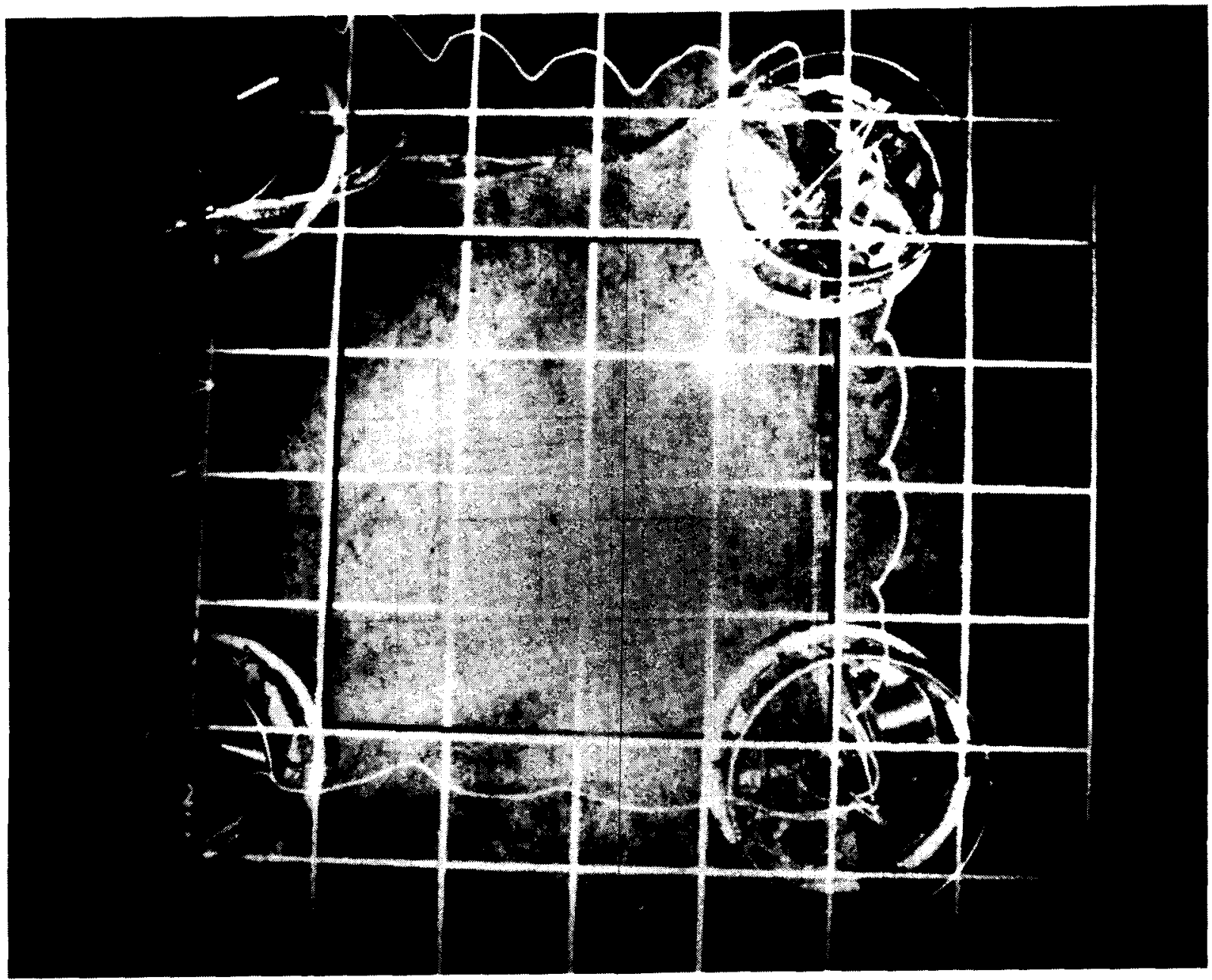

Figure 2-10: Photograph of 3D machine traversing a square path under position control. Each time the operator pressed a sequencing button. the machine advanced from one predefined position setpoint to the next. An electro-optical sensor mounted on the ceiling provided position measurements that were used by the position control servo. It took about 14 seconds to traverse the path. The white line in the photograph indicates the path of an LED attached to the top of the body.

\subsection{Discussion}

One way to view the $3 \mathrm{D}$ control system reported in this paper, is that it is very much like two separate $2 \mathrm{D}$ systems that operate at right angles to one another. If one writes Eqs. (2.4) and (2.5) in terms of the components of $\mathrm{X}$, then one gets two sets of equations that are each like the $2 \mathrm{D}$ velocity control equation, Eq. (2.2).

Another way to view the system is as an implementation of the plane of motion idea, described by Murthy and Raibert (1983). They proposed that locomotion in 3D might be best understood by thinking in terms of a decomposition into a planar part and an extra-planar part. Their planar part of the control was just like the 
system used for a 2D one-legged system. The extra-planar part was responsible for maintaining the planarity of motion so that the planar part could operate effectively. The control algorithms described in this paper are perfectly consistent with this plane of motion approach. During each flight period, the control system uses the instantaneous forward velocity to determine the plane of motion, and chooses a foot position with respect to this plane. The position of the foot within the plane will determine the forward acceleration, while the position of the foot perpendicular to the plane of motion will determine the change in orientation of the plane of motion on the next step.

Both of these conceptualizations are correct and consistent. Both explanations can be summarized as follows. For every forward velocity there is a position for the foot that will provide no net acceleration during stance -- the forward velocity when the foot leaves the ground will be the same as the forward velocity when the foot last touched the ground. This position is a velocity fix point. The displacement of the foot from this position determines the acceleration of the system, and therefore, the change of speed and direction. The position that generates zero net acceleration is approximated equally well by the pair of perpendicular CG-prints, and by the single CG-print in the plane of motion. The difference is like the difference between representing a point in Cartesian coordinates or in polar coordinates.

For the experiments reported in this paper, $\theta_{P}=\theta_{R}=\dot{\theta}_{P}=\dot{\theta}_{R}=0$. As a result, the algorithm that controlled attitude of the body, Eq. (2.9), produced an asymmetrical oscillation of $\theta_{\mathrm{p}}$ and $\theta_{\mathrm{R}}$. The body was made erect at lift-off, but because angular momentum must be conserved during flight, motion of the leg caused motion of the body. Furthermore, at the beginning of stance, the body was suddenly made erect. It should be possible to reduce the asymmetry of $\theta_{P}$ and $\theta_{R}$ oscillations, and to eliminate the sudden erection of the body at touchdown, by choosing the roll and pitch setpoints to satisfy:

$$
\begin{array}{ll}
\mathrm{J}_{\mathrm{BODY}} \theta_{\mathrm{P}}+\mathrm{J}_{\mathrm{LBG}} \boldsymbol{\varphi}_{\mathrm{P}}=0 & \mathrm{~J}_{\mathrm{BODY}} \dot{\theta}_{\mathrm{P}}+\mathrm{J}_{\mathrm{LBG}} \dot{\theta}_{\mathrm{P}}=0 \\
\mathrm{~J}_{\mathrm{BODY}} \theta_{\mathrm{R}}+\mathrm{J}_{\mathrm{LBG}} \boldsymbol{\varphi}_{\mathrm{R}}=0 & \mathrm{~J}_{\mathrm{BODY}} \dot{\theta}_{\mathrm{R}}+\mathrm{J}_{\mathrm{LEG}} \dot{\theta}_{\mathrm{R}}=0
\end{array}
$$

where

$\mathrm{J}_{\text {BODY }}$ is the moment of inertia of the body about the hip,

$\mathrm{J}_{\mathrm{LEG}}^{\mathrm{BODY}} \quad$ is the average moment of inertia of the leg about the hip

$\theta_{P}, \theta_{R} \quad$ are the pitch and roll angles of the body, and

$\dot{\varphi}_{P}, \varphi_{R} \quad$ are the pitch and roll angles of the leg.

These equations specify that the attitude of the leg and body remain vertical, and that the average angular rate of the system remain zero. We have not yet tested this approach extensively.

In the last section we reported a failure to control the facing direction of the machine. The sources of yaw torque available in the machine we built were inadequate to overcome the disturbance torque generated by the umbilical cable. As a practical problem, this failure did not interfere with the experiments, but was finessed by doing a little extra computing. Moreover, yaw control is not likely to be a difficult problem for legged vehicles. Useful legged vehicles will not have umbilical cables, and their legs can be designed to 
generate yaw torques directly if required. For systems with more than one leg, legs can act together to generate substantial yaw torque.

The decision to explore locomotion in a system with just one leg was motivated by our desire to focus on balance as the primary research issue. A one-legged system must balance in order to remain upright during locomotion, it must have a ballistic phase. on each step, and it can move with substantial velocity. These characteristics are not found in most previous walking machines, which are statically stable. It was also our goal to avoid the difficult problem of coordinating many legs until we had more experience with one.

While the primary purpose of using a one-legged machine for these experiments was to focus on balance, an additional goal was to develop a model that could explain the behavior of more complicated systems that run. If we ignore the third dimension, generalizing from the one-legged machine to the two-legged hopping kangaroo is very easy. A direct comparison can be made between the motions of the hopping machine's one leg and the motions of the kangaroo's pair of legs. The primary difference is that the kangaroo uses its tail to heip compensate for the large sweeping motions of the legs, so that the body need not react by pitching so much on each hop. The control system could still regulate hopping height, body attitude, and velocity as before.

Many characteristics of the running biped are also similar to a system that runs on one leg, including the alternation between stance and flight the regular vertical oscillations, and the periods of one-legged support. In the case of the biped, the two legs swing in opposite directions, making pitching motions of the body and a tail unnecessary. Think of a biped as a hopping machine that substitutes a different leg on each stride. The same algorithms that were used to control the 3D hopping machine could be used to control a biped without modification. Extensions of this approach to systems with more legs is underway. See Chapter 3 for preliminary results.

\subsection{Summary}

This paper presents a set of algorithms for control of a machine that runs and balances on one leg in 3D, and it describes experiments that evaluate their performance. The goal was to explore the fundamental problems of active balance in dynamic legged systems.

We found that the algorithms designed for control of a planar one-legged system generalized to $3 \mathrm{D}$ with surprising ease. As in $2 \mathrm{D}$, the control problem decomposed into three separate parts that are each synchronized by the ongoing behavior of the machine. One control part regulates the forward running velocity of the system and the rate of turn by placing the foot a specific distance in front of, and to the side of the hip as the hopping machine approaches the ground on each step. The second control part maintains the body in an erect posture by servoing the hip during stance. The third control part determines hopping height by choosing a fixed amount of energy to inject on each hopping cycle. The control algorithms are very simple because these three functions are treated independently. 
Experiments showed that the 3D hopping machine balanced without external support, while hopping in place and while traveling about the laboratory. It tracked a velocity ramp and sudden changes in desired direction with $0.25 \mathrm{~m} / \mathrm{sec}$ accuracy. At higher speeds the system consistently ran slower than specified due to inaccuracies in estimating the CG-print. Maximum recorded speed was $2.2 \mathrm{~m} / \mathrm{sec}(4.8 \mathrm{mph})$. In position control the system determined the position of the machine in the laboratory by integrating the estimated running velocity. With a stationary position setpoint, the machine could hop in place with about $\pm 0.25 \mathrm{~m}$ accuracy. The machine also traversed a square path, but the path accuracy suffered due to interference from forces generated by the umbilical cable. The system continued to balance while the experimenter delivered a sudden jab to the machine's body with his hand.

While legged vehicles with just one leg could very well turn out to have utility in their own right, the real purpose of these experiments was to explore the fundamental principles of balance in a simple legged system. A system with just one leg was a good choice for these experiments because balance is of paramount importance to its locomotion, and because the problem of coordinating many legs was avoided. The results of this work may help us to better understand both the overall behavior of legged systems that actively balance, and the individual behavior of each leg in systems with more than one. 


\subsection{Appendix A: Physical Parameters of 3D One-Legged Machine}

Parameter

Overall Height

Overall Width

Hip Height

Total Mass (Body \& Leg)

Unsprung Leg Mass

Ratio: Body Mass to

Unsprung Leg Mass

Body Moment of Inertia

Leg Moment of Inertia

Ratio: Body Moment of Inertia to

Leg Moment of Inertia

Leg Vertical Motion

Stroke

Ideal No-Load Stroke Time

Static Force

Ratio: Static Force to Weight

Theoretical Max. Work per Stroke

\section{Leg Sweep Motion}

Sweep Angle

Ideal No-Load Sweep Time

Static Torque

Theoretical Max. Work per Stroke
Metric Units

$1.10 \mathrm{~m}$

$0.76 \mathrm{~m}$

$0.58 \mathrm{~m}$

$17 \mathrm{~kg}$

$0.91 \mathrm{~kg}$

$18: 1$

$0.709 \mathrm{~kg}-\mathrm{m}^{2}$

$0.111 \mathrm{~kg}-\mathrm{m}^{2}$

$6.4: 1$

$0.25 \mathrm{~m}$

$0.031 \mathrm{~s} @ 620 \mathrm{kPa}$

$630 \mathrm{~N} @ 620 \mathrm{kPa}$

3.7:1

$160 \mathrm{~N}-\mathrm{m}$

$1.00 \mathrm{rad} / 0.71 \mathrm{rad}$

$0.069 \mathrm{~s} @ 14 \mathrm{mPa}$

$90 \mathrm{~N} \cdot \mathrm{m} / 136 \mathrm{~N} \cdot \mathrm{m} @ 14 \mathrm{mPa}$

$83 \mathrm{~N}-\mathrm{m}$
English Units

43.5 in

30.0 in

23.0 in

$38 \mathrm{lbm}$

$2.0 \mathrm{lbm}$

18:1

$2420 \mathrm{lbm}-\mathrm{in}^{2}$

$380 \mathrm{lbm}-\mathrm{in}^{2}$

$6.4: 1$

10.0 in

0.031 s@90 psig

140 lb@90 psig

3.7:1

$1400 \mathrm{lb}$-in

$57^{\circ} / 41^{\circ}$

0.069 s@2000 psig

800 lb-in/1200 lb-in@2000 psig

$740 \mathrm{lb}$-in 


\subsection{Appendix B: Kinematics of 3D Machine}

We define three coordinate frames $\{\mathbf{W}\},\{\mathbf{H}\}$, and $\{B\}$. Frame $\{\mathbf{W}\}$ is the base coordinate frame, which is fixed in the laboratory. The origin of frame $\{\mathbf{H}\}$ moves with the hip, but its orientation remains parallel to $\{W\}$. Think of frame $\{H\}$ as attached to the innermost gimbal of the gyroscope. For $\{W\}$ and $\{H\}, z$ is aligned with the gravity vector, and positive upward. Frame $\{\mathbf{B}\}$ is fixed to the body. Its origin also moves with the hip, but $\{B\}$ changes orientation with respect to $\{W\}$ and $\{H\}$. The Euler angles that specify the orientation of $\{B\}$ are $\left(\theta_{Y}, \theta_{R}, \theta_{P}\right)$. The hip and leg actuators determine the position of the foot in frame $\{B\}$.

Let the vector ${ }^{\mathrm{P}} \mathbf{X}$ be a vector $[\mathrm{X}, \mathrm{y}, \mathrm{z}, 1]^{\mathrm{T}}$ expressed in coordinate frame $\{\mathbf{P}\}$. The transformation from coordinate frame $\{\mathrm{B}\}$ to $\{\mathbf{H}\}$ :

$$
\begin{aligned}
& { }_{X}{ }^{H}={ }_{B}^{H} T^{B} X \\
& { }_{B}^{H} T= \\
& {\left[\begin{array}{l}
\cos \left(\theta_{P}\right) \cos \left(\theta_{Y}\right) \cdot \sin \left(\theta_{P}\right) \sin \left(\theta_{R}\right) \sin \left(\theta_{Y}\right) \\
\cos \left(\theta_{P}\right) \sin \left(\theta_{Y}\right)+\cos \left(\theta_{Y}\right) \sin \left(\theta_{P}\right) \sin \left(\theta_{R}\right) \\
\cos \left(\theta_{R}\right) \sin \left(\theta_{P}\right) \\
0
\end{array}\right.}
\end{aligned}
$$$$
\begin{array}{ll}
-\cos \left(\theta_{R}\right) \sin \left(\theta_{Y}\right) & -\cos \left(\theta_{P}\right) \sin \left(\theta_{R}\right) \sin \left(\theta_{Y}\right)-\cos \left(\theta_{Y}\right) \sin \left(\theta_{P}\right) \\
\cos \left(\theta_{R}\right) \cos \left(\theta_{Y}\right) & \cos \left(\theta_{P}\right) \cos \left(\theta_{Y}\right) \sin \left(\theta_{R}\right)-\sin \left(\theta_{P}\right) \sin \left(\theta_{Y}\right) \\
-\sin \left(\theta_{R}\right) & \cos \left(\theta_{P}\right) \cos \left(\theta_{R}\right) \\
0 & 0
\end{array}
$$

Transformation from frame $\{H\}$ to $\{B\}$ :

$$
\begin{aligned}
& { }_{\mathrm{X}}^{\mathrm{B}}={ }_{\mathrm{H}}^{{ }_{\mathrm{B}} \mathrm{H}} \mathrm{X} \\
& { }_{\mathrm{H}}^{\mathrm{B}} \mathrm{T}= \\
& {\left[\begin{array}{l}
\cos \left(\theta_{\mathrm{P}}\right) \cos \left(\theta_{\mathrm{Y}}\right)-\sin \left(\theta_{\mathrm{P}}\right) \sin \left(\theta_{\mathrm{R}}\right) \sin \left(\theta_{\mathrm{Y}}\right) \\
-\cos \left(\theta_{\mathrm{R}}\right) \sin \left(\theta_{\mathrm{Y}}\right) \\
-\cos \left(\theta_{\mathrm{P}}\right) \sin \left(\theta_{\mathrm{R}}\right) \sin \left(\theta_{\mathrm{Y}}\right)-\cos \left(\theta_{\mathrm{Y}}\right) \sin \left(\theta_{\mathrm{P}}\right) \\
0
\end{array}\right.}
\end{aligned}
$$$$
\left.\begin{array}{lll}
\cos \left(\theta_{\mathrm{P}}\right) \sin \left(\theta_{\mathrm{Y}}\right)+\cos \left(\theta_{\mathrm{Y}}\right) \sin \left(\theta_{\mathrm{P}}\right) \sin \left(\theta_{\mathrm{R}}\right) & \cos \left(\theta_{\mathrm{R}}\right) \sin \left(\theta_{\mathrm{P}}\right) & 0 \\
\cos \left(\theta_{\mathrm{R}}\right) \cos \left(\theta_{\mathrm{Y}}\right) & -\sin \left(\theta_{\mathrm{R}}\right) & 0 \\
\cos \left(\theta_{\mathrm{P}}\right) \cos \left(\theta_{\mathrm{Y}}\right) \sin \left(\theta_{\mathrm{R}}\right)-\sin \left(\theta_{\mathrm{P}}\right) \sin \left(\theta_{\mathrm{Y}}\right) & \cos \left(\theta_{\mathrm{P}}\right) \cos \left(\theta_{\mathrm{R}}\right) & 0 \\
0 & 0 & 1
\end{array}\right]
$$ 

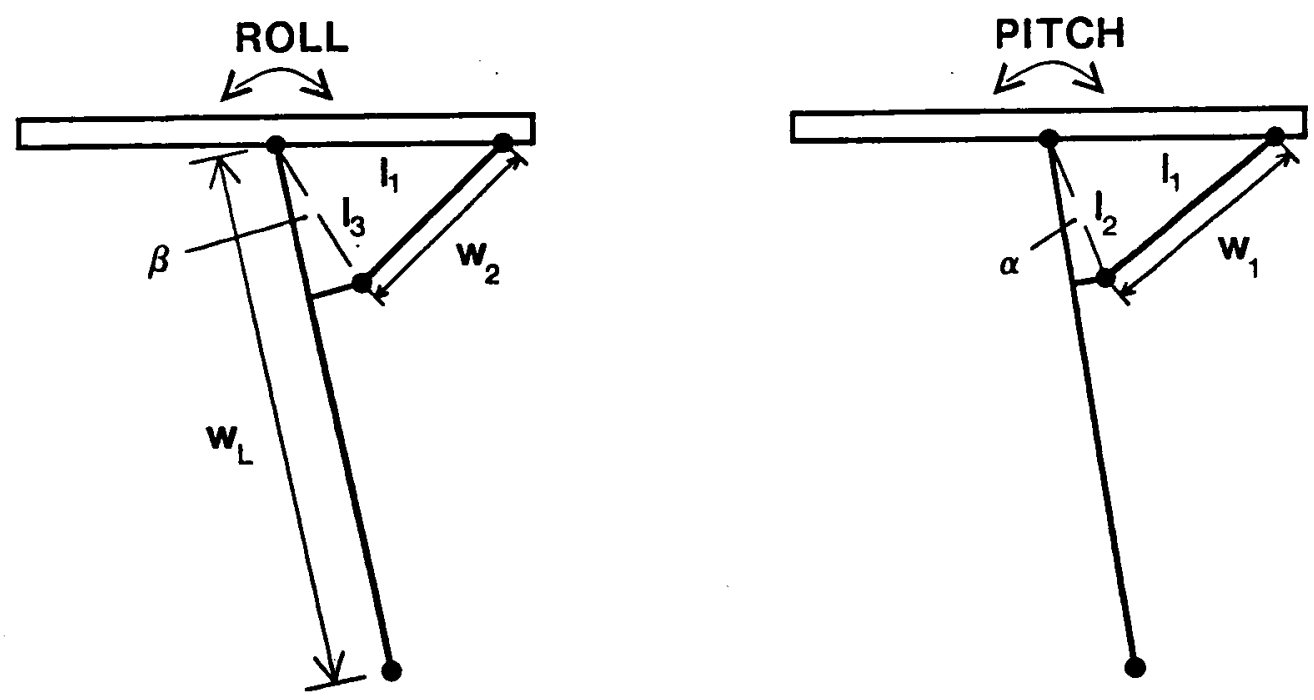

Figure 2-11: Diagram that shows kinematics of actuators, hip, and leg. Actuator lengths are represented by $w_{1}$ and $w_{2}$, and leg length by $w_{L} \cdot l_{1}=0.345 \mathrm{~m}, l_{2}=0.0508 \mathrm{~m}, l_{3}=0.0762 \mathrm{~m}, \alpha=8.46 \mathrm{deg}, \beta=27.28$

Relationships between actuator lengths and position of foot with respect to the body in frame $\{\mathbf{B}\}$. See Fig. 2-11. First the forward solution:

$$
\begin{aligned}
& { }^{\mathrm{B}} \mathrm{X}_{\mathrm{F}}={ }_{\mathrm{A}}^{\mathrm{B}} \mathrm{T}(\mathrm{W}) \\
& { }_{\mathrm{A}}^{\mathrm{B}} \mathrm{T}(\mathrm{W})= \\
& x=\sqrt{w_{L} \cos \left\{\operatorname{Arccos}\left[\frac{w_{1}^{2}-1_{1}{ }^{2}-1_{2}^{2}}{-2 l_{1} l_{2}}\right]+\alpha\right\}} \\
& y=\sqrt{w_{1} \cos \left\{\operatorname{Arccos}\left[\frac{w_{2}{ }^{2}-1_{1}{ }^{2}-1_{3}{ }^{2}}{-21_{1} \cdot 1_{3}}\right]+\beta\right.} \\
& z=\sqrt{w_{L}^{2}-x^{2}-y^{2}} \\
& { }^{B} \mathbf{X}_{F}=[x, y, z, 1]^{\mathrm{T}}
\end{aligned}
$$

The inverse solution:

$$
\begin{aligned}
& \mathbf{W}={ }_{A}^{\mathrm{B}} \mathrm{T}\left({ }^{\mathrm{B}} \mathrm{X}_{\mathrm{F}}\right) \\
& \mathbf{W}=\left[\mathrm{w}_{1}, \mathrm{w}_{2}, \mathrm{w}_{\mathrm{L}}\right]^{\mathrm{T}} \\
& \mathbf{w}_{1}=\mathrm{l}_{1}^{2}+\mathrm{l}_{2}^{2}-21_{1} 1_{2} \cos \left[\operatorname{Arccos}\left[\frac{\mathrm{x}}{-\mathrm{w}_{L}}\right]-\alpha\right]
\end{aligned}
$$




$$
\begin{aligned}
& w_{2}=1_{1}^{2}+1_{3}^{2}-21_{1} l_{3} \cos \left[\operatorname{Arccos}\left[\frac{y}{-w_{L}}\right]-\beta\right] \\
& w_{L}=-\sqrt{x^{2}+y^{2}+z^{2}}
\end{aligned}
$$

The overall transformations between actuator variables and foot position in $\{\mathrm{H}\}$ :

$$
\begin{aligned}
& { }^{\mathrm{H}} \mathbf{X}_{F}={ }_{B}^{\mathrm{H}} \mathbf{T}{ }_{A}^{\mathrm{B}} \mathrm{T}(\mathbf{W})=F^{-1}(\mathbf{W}) \\
& \mathbf{W}={ }_{B}^{\mathrm{A}} \mathbf{T}\left({ }_{\mathbf{H}}^{\mathrm{B}} \mathrm{T}^{\mathrm{H}} \mathbf{X}_{\mathrm{F}}\right)=\mathbf{F}\left({ }^{\mathrm{H}} \mathbf{X}_{\mathrm{F}}\right)
\end{aligned}
$$




\title{
3. Control of Trotting and Bounding for a Simple Planar Model
}

\author{
Karl N. Murphy
}

\subsection{Abstract}

This report describes control algorithms for the running and balance in a multi-legged planar model. The model has two legs and represents the lateral half of a quadruped. We call the model the planar dog. We decompose control of the planar dog into three parts. The first part controls vertical motions, the second part controls forward velocity, and the third part controls body attitude. The three parts of the control act independently of each other, but are coordinated by a finite state sequencer. By simulating the model we have shown two variations of the control algorithms that result in two different gaits. The first variation uses all three parts of the control system, and causes the planar dog to trot. The second variation controls vertical and forward motion but does not use attitude control, and causes the planar dog to bound.

\subsection{Introduction}

Animals, including man, demonstrate feats of great mobility. Their legs allow them to move swiftly over terrain that is either too rough, too slippery, or too soft for wheeled or tracked vehicles. The advantages of using legs for locomotion are great, but our understanding of how animals use their legs is limited.

Previous work on walking has focused on bipeds. Hemami and his co-workers (Ceranowicz, 1979; Hemami and Cvetkovic, 1976; Hemami and Farnsworth, 1977), have developed a five link planar biped. The model is controlled using state feedback. The reference positions and velocities come from a movie of a human walking. Vukobratovic and his co-workers (Juricic and Vukobratovic, 1972; Vukobratovic and Stepaneko, 1973; Vukobratovic and Okhotsimskii, 1975) modeled a walking biped that balances by manipulating the projected center of gravity and the support area provided by the feet. Others use a pre-planned sequence for quasi-static motion (Kato et al., 1981) and tabular control to maintain balance (Miura and Shimoyama, 1980). These bipeds are all patterned after the geometry of the human, with a small hip separation. The bipeds also keep one foot on the ground at all times.

Raibert and his co-workers have studied balance in running. They have modeled and built legged machines that hop on one springy leg. By using only one leg, they avoided the problem of coordinating many legs. The control system only worries about the problem of balance.

The controller of these one-legged machines used three separate servo loops to control hopping height, body attitude, and forward velocity. Hopping height was controlled by delivering a thrust with the leg when the body reached its lowest vertical position during each hop. This thrusting resupplied the energy lost to friction 
and ground impact. Body attitude was controlled by torquing the hip during stance when friction keeps the foot from moving. A linear servo drove the body level. During flight, the forward velocity controller swung the foot to a position in front of the hip. The foot position determined the forward acceleration during the next period of stance. This three part controller successfully controlled $2 \mathrm{D}$ and $3 \mathrm{D}$ one-legged systems.

The success and simplicity of the control systems used for the one-legged hoppers have encouraged us to study multi-legged systems. Since the problem of leg coupling and coordination was completely avoided with the one-legged hoppers, several questions arise. What is needed to coordinate the actions of several legs? Can we generalize the control of a one-legged machine to control of a multi-legged machine? Is balance of a multi-legged machine similar to balance of the one-legged hoppers?

In order to answer these questions we have devised a planar model with two legs. The model has a long body with hips at each end. By definition, the model is a biped since it only has two legs. However, our intention is to represent the lateral half of a quadruped. With only two legs and planar motion, the model is not so complicated as a quadruped moving in three dimensions, but it still allows us to explore the basic behavior of a quadruped.

We are exploring how ideas originally formulated in the context of a system with one leg can be generalized to the multi-legged case. Our purpose is to understand how systems with a long body run, and how to coordinate the actions of several legs. We expect studying this model to help us learn how to control four-legged systems

\subsection{Model}

When considered dynamically, legged systems have two primary components, the body and the legs. The body usually contains most of the mass and provides a place to connect the legs. The actions of the legs must control the position and velocity of the body. The legs transmit ground forces and moments to the body. Legs change length and orientation with respect to the body.

The model, shown in Fig. 3-1, has a rigid body of mass $\mathrm{M}_{3}$ and moment of inertia $\mathrm{I}_{3}$. The legs attach to the body a distance $r_{3}$ from the center of gravity of the body. Each articulated leg has two links that are modeled as uniform rods of length $\mathrm{D}$. The lower links have mass $\mathrm{M}_{1}$ and moment of inertia $\mathrm{I}_{1}$. The upper links have mass $\mathrm{M}_{2}$ and moment of inertia $\mathrm{I}_{2}$. The simulation parameters, which are presented in the Appendix, were chosen to match those we would obtain if we were to build such a machine.

Torque actuators drive the hip joints. While a foot is in the air, the hip actuator orients the leg with respect to the body, driving the leg to a desired angle. While a foot is on the ground, the hip actuator sweeps the leg backward driving the body forward.

A position actuator in series with a spring drives each knee. See Fig. 3-2. The position servos in the knees 


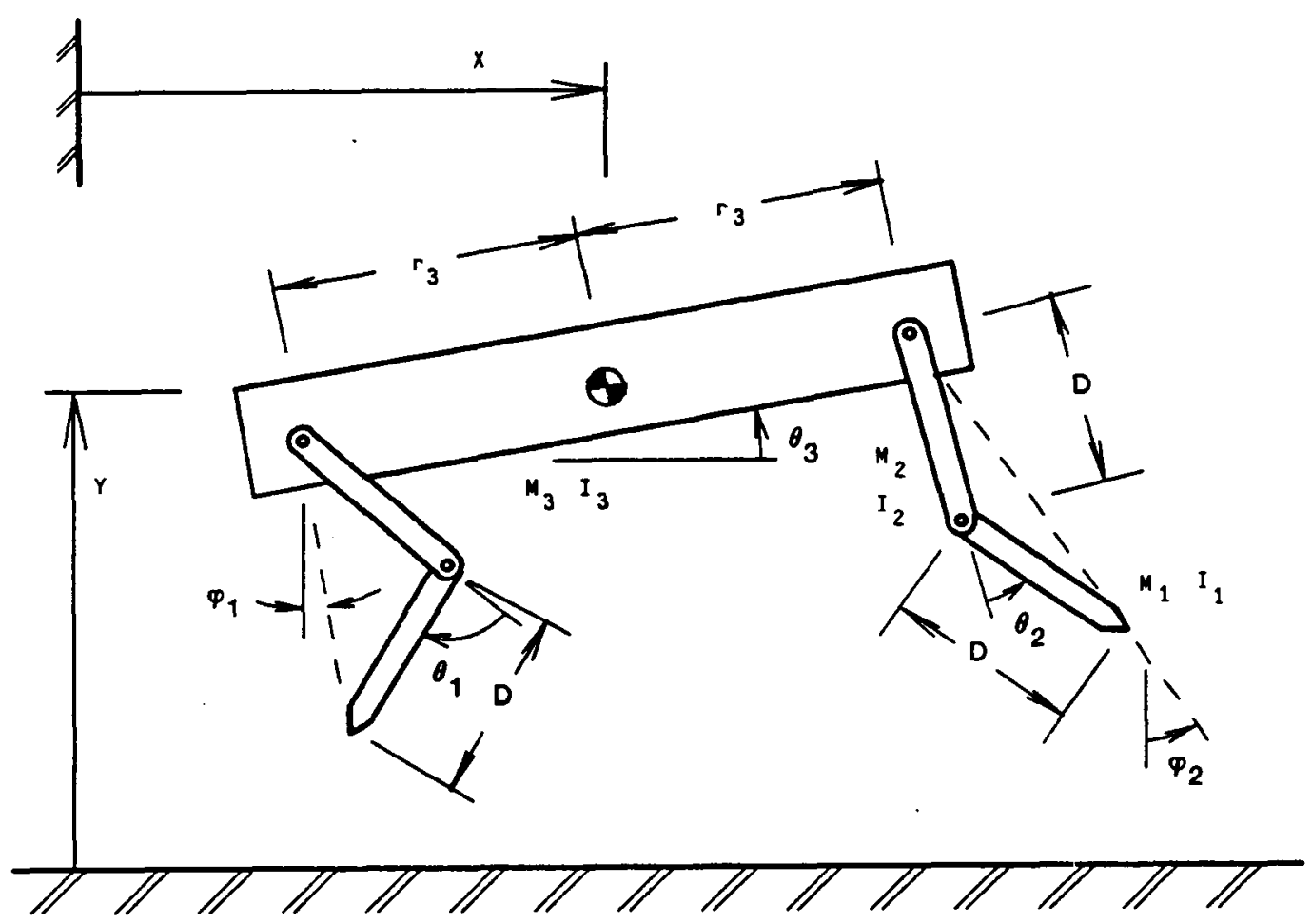

Figure 3-1: The planar dog: the model used for simulation and control. The body and the two links of both legs have mass and moment of inertia. All four joints are simple hinges. Control torque is generated at both hips. Each knee is driven by a position actuator in series with a spring, (see Fig. 3-2). The ground is springy in two dimensions. The model is restricted to motion in a plane. See the Appendix for the values of the simulation parameters.

excite and maintain vertical oscillations. They can also be used to control the body attitude. For simplicity, the actuators are assumed to be perfect position servos that can act instantaneously. The spring and actuator configuration, shown in Fig. 3-2, uses a linear spring and actuator. This produces a torque such that the thrust is proportional to the amount the leg has contracted plus the length of the position actuator. The static force required to compress the leg to a given length, $\mathrm{L}$, is called the leg thrust $\mathrm{T}$.

$$
\mathrm{T}=\mathrm{K}_{\mathrm{S}}\left[\mathrm{L}_{0}-(\mathrm{L}-\mathrm{P})\right]=\mathrm{K}_{\mathrm{S}}\left(\mathrm{L}_{0}-\mathrm{L}\right)+\mathrm{K}_{\mathrm{S}} \mathrm{P}
$$

where

$\mathrm{K}_{\mathrm{S}}$ is the spring constant

$\mathrm{L}_{0}$ is the free length of the spring

$P$ is the length of the position actuator.

The thrust can be decomposed into two parts. The first part, $\mathrm{K}_{\mathrm{S}}\left(\mathrm{L}_{0}-\mathrm{L}\right)$, is determined solely by the length of the leg and is called passive leg thrust. The second part, $\mathrm{K}_{\mathrm{S}} \mathrm{P}$, is determined by the length of the position 


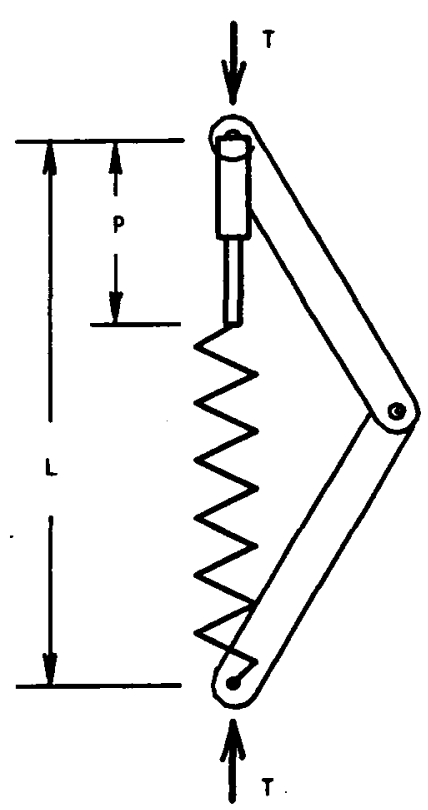

Figure 3-2: The knee actuator and spring arrangement. A given change in length of the position actuator always results in a proportional change in the total thrust, $T$.

actuator, and is called active leg thrust. The total thrust is controlled by changing the length of the position actuator. The active thrust can be positive or negative, but the total thrust can never be negative since no "glue" holds the foot to the ground.

There is a mechanical stop on the knee which is modeled as a stiff spring and damper. The stop keeps the leg from extending too far by preventing the knee angle, $\theta$, from becoming too small. The stop influences the knee only when the knee angle is less than a certain amount, $\theta<\theta_{\min }$. The location of the stop, $\theta_{\min }$, does not depend on the position of the knee actuator. Extending the actuator while the knee is against the stop does not extend the leg but instead compresses the knee spring against the stiff mechanical stop. The leg spring is at rest when the knee is against the stop and the position actuator is at zero length.

The ground is modeled as a two dimensional spring.and damper. One dimension of the spring acts vertically while the other acts horizontally. The ground produces forces on a foot only when the foot is on the ground, $y_{\text {foot }}<0$. The vertical component of the ground forces are only positive, acting upward. Each time a foot touches the ground, the zero position of the horizontal spring is reset to the point of touch-down. The coefficient of friction is assumed to be large enough to prevent the foot from slipping. The compliance of the ground incorporates any compliance that would normally be associated with the legs and feet of the system.

We find that the model oscillates in two basic modes that correspond to gaits. The first mode is a vertical oscillation. The body stays level as it bounces up and down. This is trotting. The other mode is a rocking 
oscillation. The center of gravity moves very little as the system rotates back and forth, bouncing on one foot and then the other. This is bounding. As long as the legs sweep back and forth correctly, the forward motion has little effect on either of these two oscillations over a large range of running speeds.

\subsection{Control}

Control of the planar dog has been decomposed into three separate parts, one for vertical motion, one for forward motion, and one for angular motion. The three parts act independently but are coordinated by a finite-state sequencer.

Vertical motion is initiated and maintained by thrusting with both legs. Velocity control requires two separate actions. When a foot is in the air, the leg swings forward a specific distance. When a foot is on the ground, hip torques that are proportional to the velocity error sweep the leg backward, driving the body forward. Attitude control tries to keep the body level by controlling the difference in leg thrust. A linear servo is used to drive the body level.

We have explored two variations of the control system. The first variation uses all three control parts: vertical height control, forward velocity control, and attitude control. The result is trotting. The second variation only uses vertical height control and forward velocity control. It does not explicitly control the attitude of the body. The result is bounding. These gaits are explained in the results section.

The controllers are coordinated by a finite state sequencer that relies on the state of the feet, hips, and body. Each foot undergoes a period when in the air, followed by a period on the ground. These periods are known as flight and stance, respectively, and the transitions between the periods are known as lift-off and touch-down. The center of gravity of the body rises and reaches a maximum height known as top, falls, reaches a minimum height known as bottom, and then rises again. There is a top and bottom associated with each of the two hips, as well as with the center of gravity of the body.

The relative timing of state transitions is not constant, but varies from gait to gait. For example, when bounding, one foot will touch-down and lift-off while the other foot remains in the air. A transition may occur twice in each cycle. For example, when bounding, the center of gravity of the body peaks and bottoms twice in each cycle.

We now examine the three parts of the control system in more detail.

\subsubsection{Vertical Control}

Control of hopping height is very simple for a one-legged hopping machine. Thrusting with the leg resupplies the energy lost on each hop due to friction and ground impact. The planar dog uses a similar method. A leg thrusts a constant amount when its hip reaches bottom. Thrusting is accomplished by lengthening the leg position actuator. The time when the thrust is delivered is determined independently for each leg. 


\subsubsection{Velocity Cont rol}

The concept of the CG-print, the locus of points over which the center of gravity passes while the foot is on the ground, was used in the control of one-legged hopping machines. For a multi-legged system, we also use the idea of a hip-print. The hip-print is the locus of points over which the hip passes while its foot is on the ground.

When the feet are placed in the center of the hip-prints, the machine spends the first half of the stance period accelerating backward and the second half accelerating forward. In this case, a system slows down during the first half of stance and speeds during up the second half. This leaves the velocity at take-off about equal to the velocity at touch-down. See Fig. 3-3.

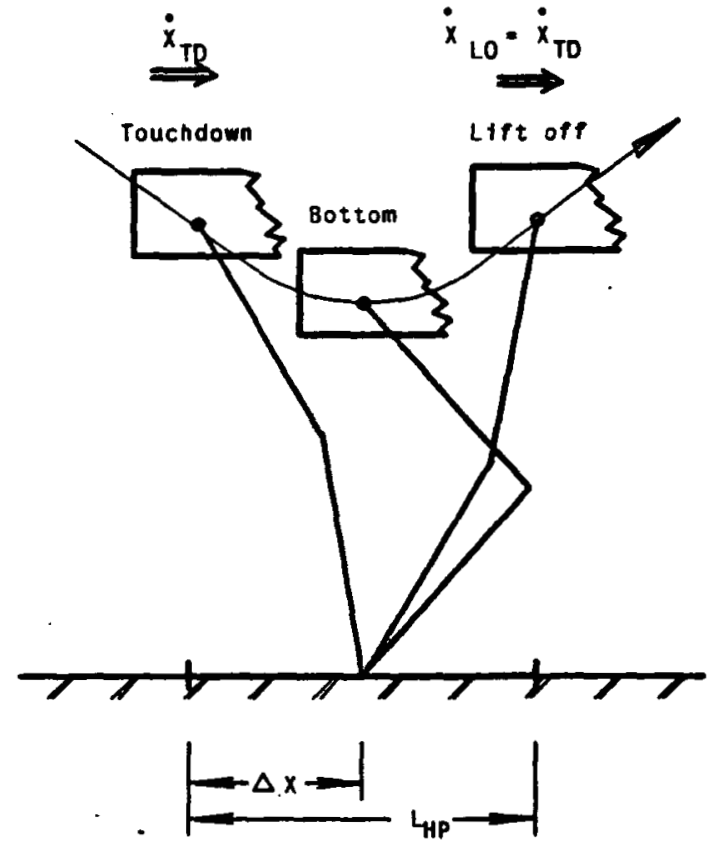

Figure 3-3: Time sequence of the rear hip shows the hip-print of length $\mathrm{L}_{\text {HP. }}$. While in the air, the foot swings in front of the hip a horizontal distance $\Delta x$. By placing the foot in the center of the hip-print, $\Delta \mathrm{x}=\mathrm{L}_{\mathrm{HP}} / 2$, the dog spends the same amount of time accelerating backward as it does accelerating forward. Forward velocity, $x$ is thus left almost unchanged.

We estimate the length of the hip-print as:

$$
\begin{aligned}
& \mathrm{L}_{\text {HP }}=\dot{\mathrm{x}} \mathrm{T}_{\text {ST }} \\
& \text { where } \\
& \dot{\mathrm{x}} \quad \text { is the forward velocity } \\
& \mathrm{T}_{\text {ST }} \text { is the duration of stance. }
\end{aligned}
$$

During flight, the foot should be positioned in front of the hip a distance 


$$
\Delta x=\frac{\dot{x} T_{S T}}{2}
$$

To accomplish this, the angle that the leg should make with the vertical is

$$
\boldsymbol{\varphi}_{\mathrm{d}}=\sin ^{-1}\left[\frac{\dot{\mathrm{x} T}}{2 \mathrm{~L}_{\mathrm{SI}}}\right]
$$

where

$\varphi_{\mathrm{d}}$ is the desired leg angle and

$\mathrm{L}_{0}$ is the rest length of the leg.

During flight, the hip torques drive the leg to the desired leg angle, $\varphi_{\mathrm{d}}$, using a linear servo:

$$
\tau=\mathrm{K}_{\mathrm{p}}\left(\varphi-\varphi_{\mathrm{d}}\right)+\mathrm{K}_{\mathrm{v}} \dot{\varphi}
$$

where

$\mathrm{K}_{\mathrm{p}}$ and $\mathrm{K}_{\mathrm{v}}$ are position and velocity gains.

This algorithm for placing the foot provides no velocity stability. The planar dog must be able to change and maintain a desired velocity. Accelerations could be accomplished by placing the foot in front of or behind the center of the hip print. This is what the one-legged hoppers do. However a better method for the planar dog, one which allows larger accelerations, is to use hip torques while a foot is on the ground. Hip torques drive the legs backward and the body forward. This torque, $\tau$, is set proportional to the error in forward velocity:

$$
\tau=K_{6}\left(\dot{x}-\dot{x}_{d}\right)
$$

where

$\dot{x}_{d} \quad$ is the desired forward velocity.

\subsubsection{Attitude Control}

Attitude control attempts to keep the body level at all times by differential thrusting with the legs. This method relies on the fact that vertical ground forces cause significant moments on the body. When the feet are positioned an equal horizontal distance from the center of gravity, the sum of the vertical ground forces affects the vertical acceleration of the body, while the difference in vertical forces affects the angular acceleration. Increasing the thrust of one leg while decreasing the thrust of the other leg produces a control moment on the body. If the magnitudes of the increase in thrust and the decrease in thrust are equal, the vertical oscillations are unaffected. 
The body is driven level using a linear servo. The active thrust of each leg changes to produce a control moment on the body. The change in length of the position actuators, $\Delta p$, is:

$$
\begin{aligned}
& \Delta \mathrm{p}= \pm\left(\mathrm{K}_{1} \theta_{3}+\mathrm{K}_{2} \dot{\theta}_{3}\right) . \\
& \text { where } \\
& \dot{\theta}_{3} \quad \text { is the body attitude } \\
& \dot{\theta}_{3} \text { is the body angular velocity } \\
& \mathrm{K}_{1} \text { is the position servo gain and } \\
& \mathrm{K}_{2} \text { is the velocity servo gain. }
\end{aligned}
$$

These changes in the length of the position servo are added to the change required by the vertical control. Differential thrust is applied only when both feet are on the ground, because an increase in thrust in one leg must be accompanied by a decrease in thrust in the other leg.

\subsection{Results}

To test these control algorithms and to evaluate their performance, we simulated behavior of the planar dog. The equations of motion were developed and then integrated numerically.

\subsubsection{Trotting}

The first variation of control uses all three control parts, vertical, forward velocity and attitude control. The attitude controller keeps the body level. The result is shown in Fig. 3-4. An initial error in body attitude of $5^{\circ}$ was corrected in one cycle.

With active attitude control, the model can accelerate quickly. In Fig. 3-5, the planar dog started with no forward velocity. In two cycles, it accelerated to the desired velocity of $2 \mathrm{~m} / \mathrm{sec}$. and maintained this speed during stable running. The four stick figures at the top of Fig. 3-5 show a complete cycle. The first figure shows the planar dog when the front foot touched down. The body angle at this point was the largest of the entire cycle. By the time the body center of gravity bottomed, shown in the second figure, the body had been driven level using differential thrust. The body was kept level during the remaining part of stance until lift-off, shown in the third figure. After lift-off, the legs were swept forward, causing the body to pitch nose downward. The forth figure shows the planar dog a little after the body center of gravity peaked.

The resulting gait is similar to the trotting of a four-legged animal. Trotting is a gait where the system bounces alternately on diagonal pairs of feet. The two pairs operate $180^{\circ}$ out of phases. Each leg in the planar dog represents one of the diagonal pairs of legs on a four-legged animal.

The speed at which the planar dog can trot is limited. As the model trots faster, the legs sweep a greater distance forward, causing the body to pitch more during flight. During the following stance period, a large differential thrust is required. When this becomes too large, the desired total thrust of the front leg becomes 


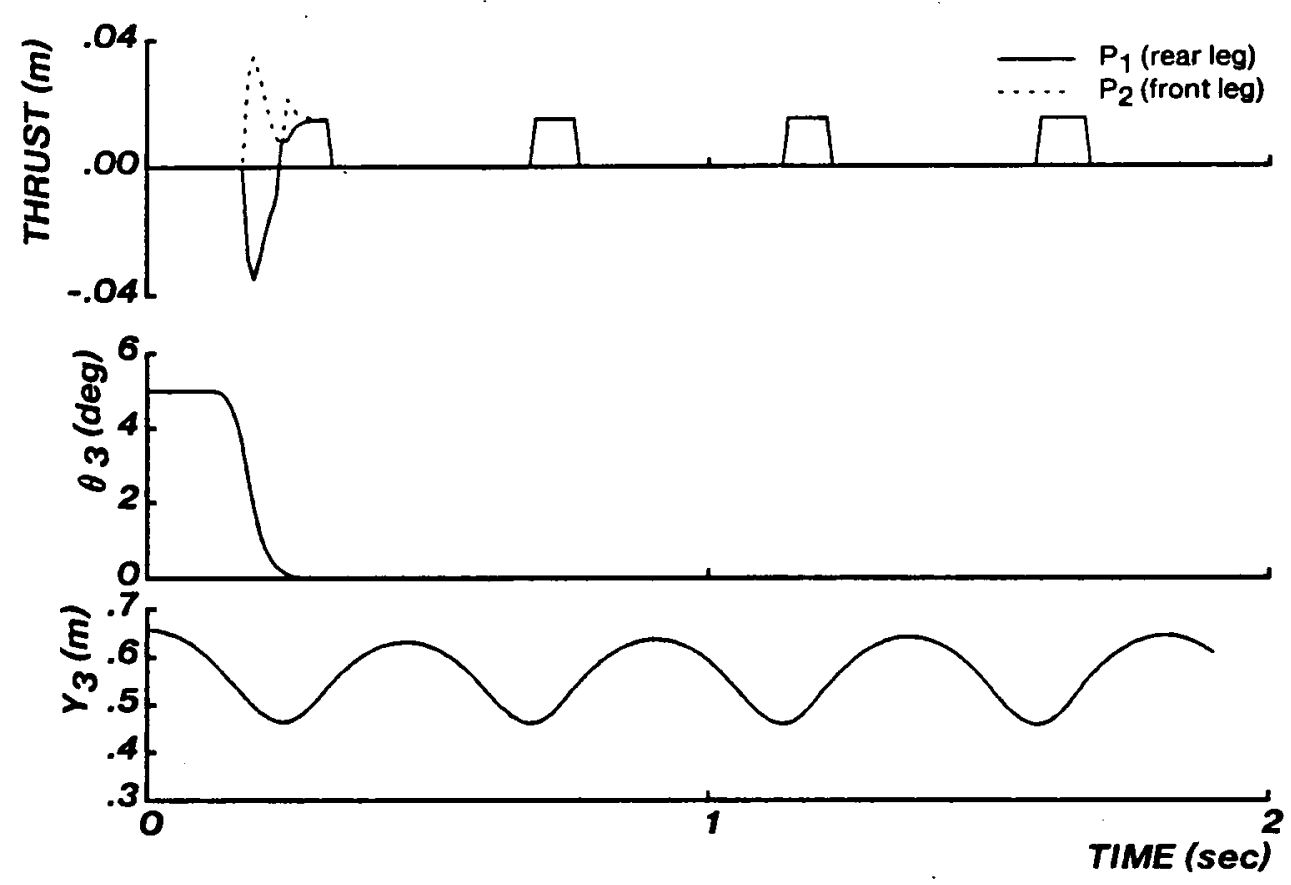

Figure 3-4: Hopping with attitude control. Differential leg thrust corrected an error in body attitude. A constant leg thrust maintained hopping height. During the first cycle, the body angle was driven to zero by the differential thrusting. On the remaining steps, thrusting maintained the vertical amplitude. The graph of thrust shows the change in the length of the position actuator of each leg which was proportional to the active thrust.

negative, which is impossible to achieve, and the foot is pulled off the ground. However, this in itself does not cause the planar dog to crash. The front foot is off the ground for a very small amount of time which has practically no effect on the behavior of the dog during that stance period. What does matter is that the timer which records the time of stance, $T_{S T}$, is reset. During the following flight phase, the front foot is swung to the estimated center of the hip-print which is calculated using the wrong $\mathrm{T}_{S T}$. The front leg swings only half the distance that it should, and during the following stance period, the planar dog trips and falls.

This problem can be corrected by several methods. Limiting the active part of thrust so that the desired total thrust does not become negative will keep the foot on the ground. A check can be made before resetting the timer. The desired body attitude can be varied during stance so that a large correction is not required at touch-down. We have not yet implemented these methods.

If the model had four legs instead of two, then the pitching of the body while trotting would be significantly reduced. There are two reasons for this. First, when one pair of legs is in the air, the other pair is on the ground keeping the body level. Second, the two pairs swing $180^{\circ}$ out of phase from each other. When one pair is swinging forward, the other pair is swinging backward. The legs can pull or push against each other without affecting the body. 

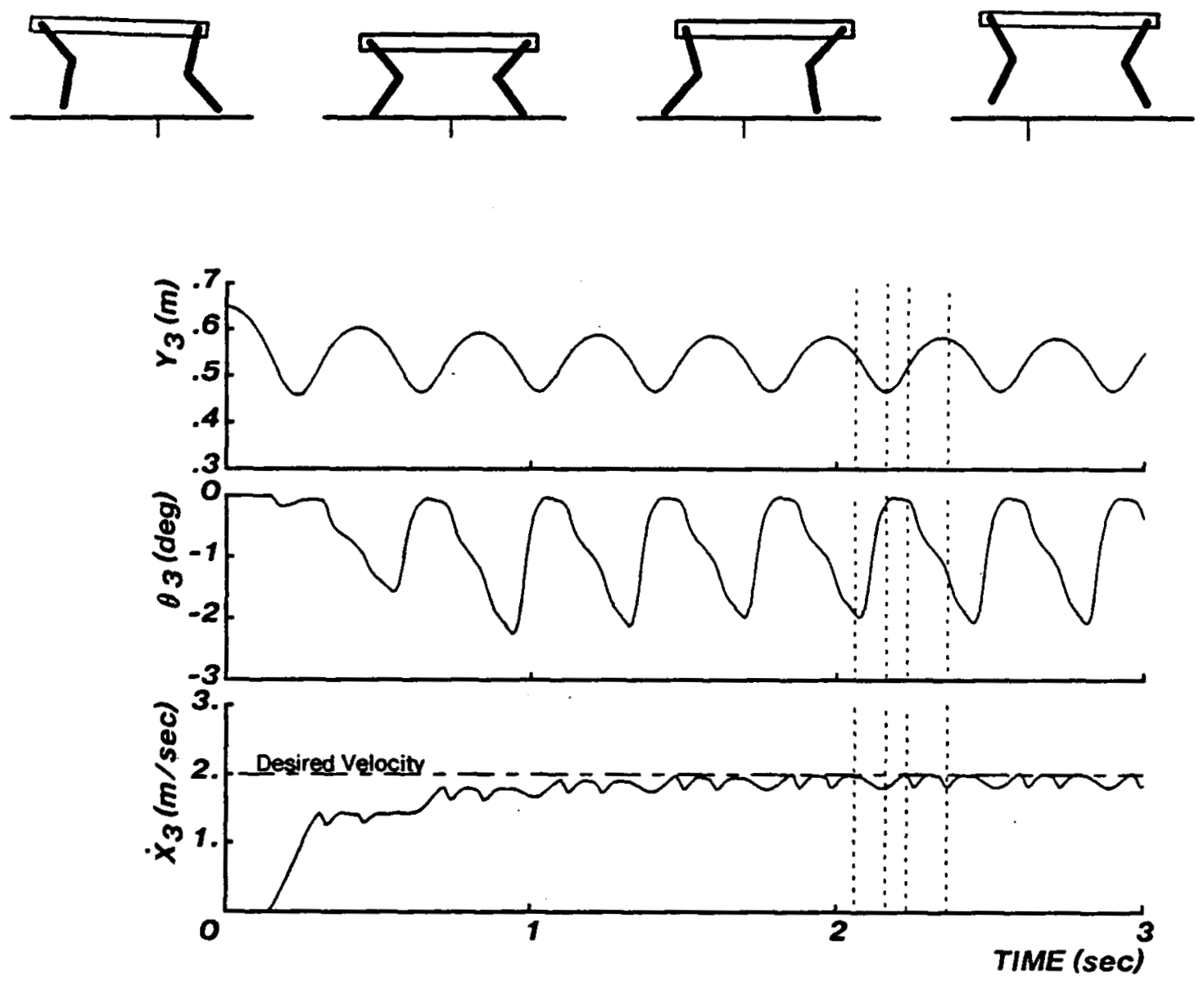

Figure 3-5: The dog trotting. The legs used a constant thrust for vertical control with differential thrust for attitude control. During flight, each foot swung forward to the estimated center of its hip-print. During stance, hip torques corrected the forward velocity. The errors in $\theta_{3}$ occurred when the feet left the ground and the legs were swept forward. This caused the body to pitch nose downward.

\subsubsection{Bounding}

The second variation of control uses only two of the three control parts, vertical and forward velocity control. This variation does not actively control the body attitude. The result is the planar dog bounding.

The behavior of the system and the stability of bounding is shown in Fig. 3-6. The dog was dropped with a small initial body attitude. A random pattern of bouncing began. After three seconds the model stabilized in a rocking oscillation. The feet were striking the ground 180 degrees out of phase from each other. The center of gravity remained nearly stationary as the body pitched back and forth. We are not sure why the dog stabilized in this manner. This stable oscillation was observed with a wide range of values of $\mathrm{I}_{3}$, the body moment of inertia. 

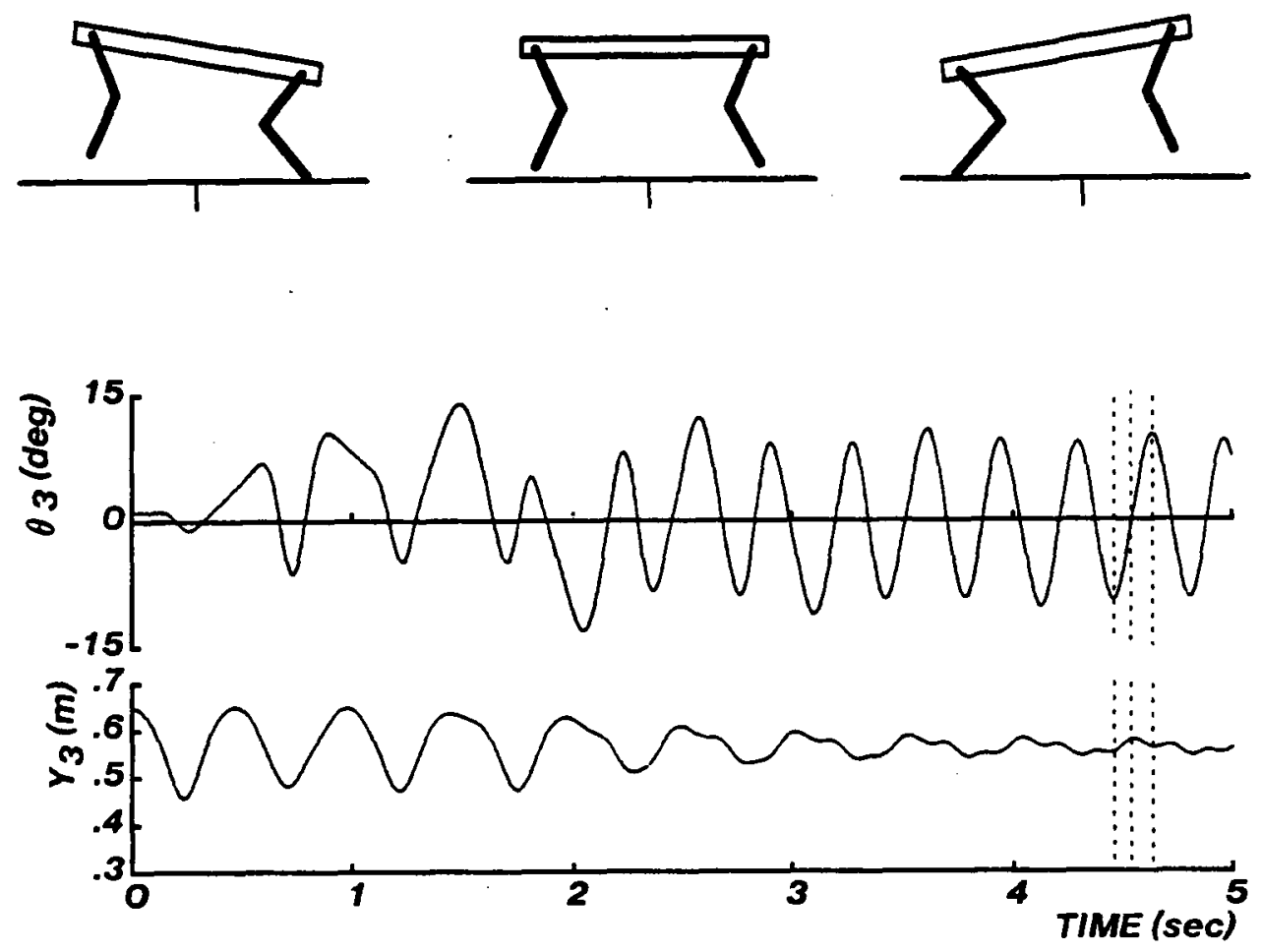

Figure 3-6: Hopping without active attitude control. Each leg thrusted independently when its hip reached bottom. A constant thrust was used. The desired velocity was set to zero. At first there was a random pattern, but it was soon replaced by a stable pattern of bouncing from one foot back onto the other. The center of gravity remained nearly stationary while the body pitches back and forth.

Control of forward velocity while bounding is shown in Fig. 3-7. With the planar dog bouncing as described, we slowly increased the desired velocity. The model accelerated up to speeds of $5 \mathrm{~m} / \mathrm{sec}(11 \mathrm{mph})$. Slow acceleration did not affect the pattern of bouncing from foot to foot.

This pattern of running closely resembles the bounding of four-legged animals. Bounding is a gait where the front pair of legs move together and the rear pair move together. The two pairs operate $180^{\circ}$ out of phase from each other, one pair touching down and lifting off while the other pair remains in the air. Each leg of our two-legged model represents a pair of legs of a bounding quadruped.

The planar dog needed to stabilize in the bouncing mode before accelerating. When large accelerations were attempted, the pattern of bouncing from foot to foot broke down. The front foot stayed on the ground and could not swing forward. This caused the planar dog to trip and fall. However, when the acceleration was small, the dog stayed in the bounding pattern and large velocities were achieved. 

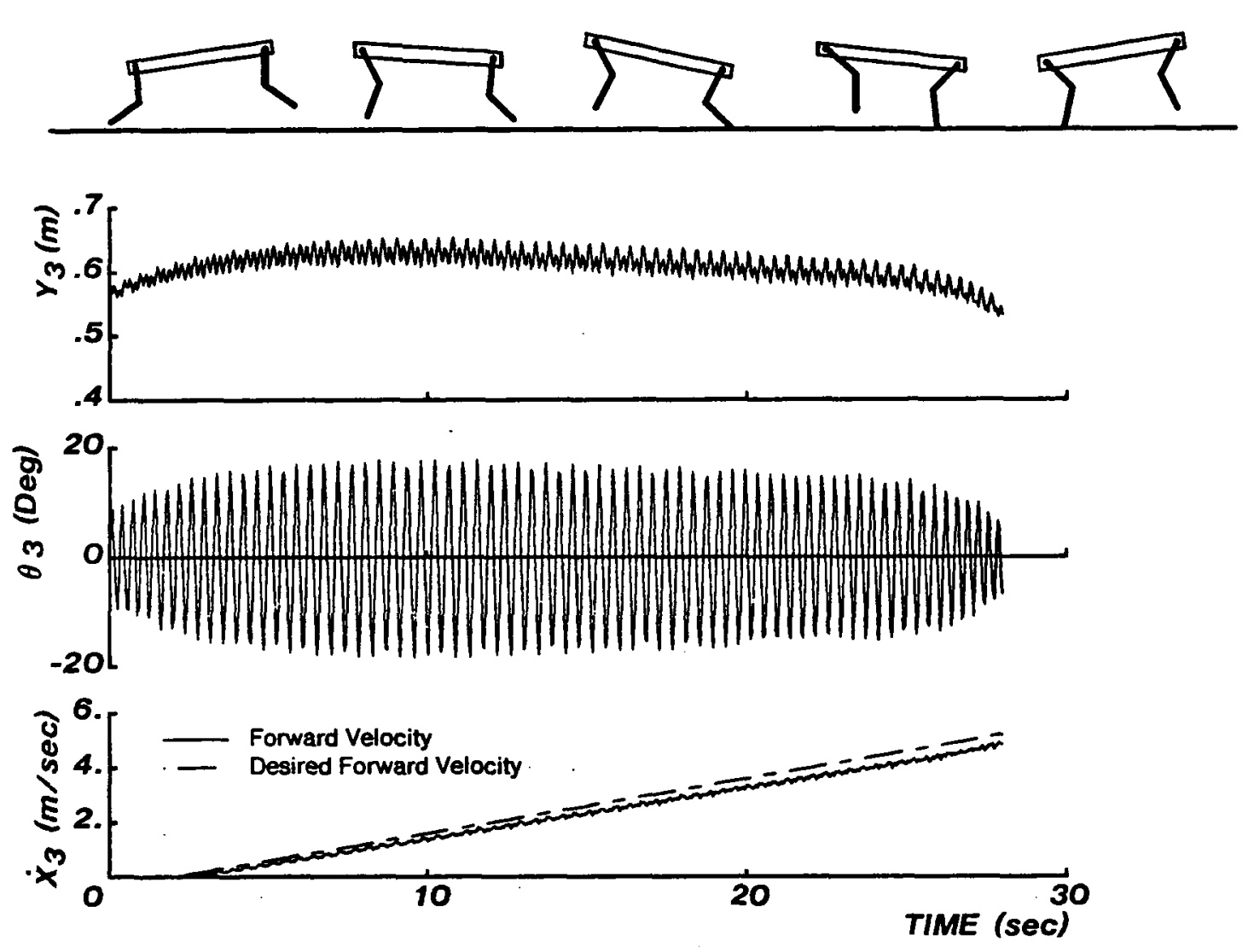

Figure 3-7: Bounding. The model stabilized in the bounding pattern and accelerated slowly to $5 \mathrm{~m} / \mathrm{sec}$ (10 mph). As the speed increased, hopping height decreased and the dog eventually stubbed its toe and tripped.

There was a limit on forward velocity. As the model bounded faster, the height of the body center of gravity decreased along with the magnitude of the angular oscillations. These two factors reduced the ground clearance of the foot as it swung forward. Eventually, the planar dog stubbed its toe, tripped, and fell. This can be corrected by either stiffening the knee springs or by contracting the leg before swinging it forward.

\subsection{Discussion}

\subsubsection{Control Strategies}

The two legs of the planar dog operate separately while bounding. The control for one leg does not depend on the actions nor the state of the other leg. Each leg can be seen as controlling its own hip. This conjures the image of a pair of one-legged hoppers joined together. If this controller were extended to a quadruped, we do not know whether all four legs could operate separately or if the two front legs and the two rear legs must be coordinated to achieve a bound. 
Unlike bounding, the control for trotting does not totally separate the actions of the two legs. The attitude control requires synchronized action from both legs. Differential thrust is used only when both feet are on the ground. Although the controllers for the vertical motion and the forward velocity separate the actions of the legs, they need not. For example, since the body is nearly level at bottom, the body center of gravity bottoms at about the same time as both hips. Thus the legs could thrust when the body bottoms without a noticeable effect.

This ability to combine the control of the two legs conjures the image of a one-legged hopping machine with a very big foot. The rear leg could be considered the heal of this large foot and the front foot could be considered the toe. The idea of combining the control of two legs into one might simplify the ideas of trotting. We would view a four-legged machine as a two-legged machine. The machine would have two big feet and would run much like a human runs on its two smaller feet.

\subsubsection{Failure of Hip Torque for Attitude Control}

We tried a second type of attitude control. This method used a similar technique to that used by the one-legged hopping machine. A hip applied torque while the corresponding foot was on the ground in an attempt to servo the body angle to zero. This method did not work for the dog. We don't know why. We believe that if the hips were much closer together and that if the moment of inertia were smaller, then attitude control using hip torque would be effective. This case is equivalent to the one-legged hopping machine.

\subsection{Conclusions}

The goal of this work is to begin exploring control of multi-legged systems. We developed a planar model that represents one lateral half of a quadruped. Having only two legs reduces the problem of coordinating many legs but still allows study of simple gaits. A long body allows the model to imitate the prominent motions of a four-legged machine.

Control of the planar dog was decomposed into three separate parts, one each for vertical motion, forward motion, and angular motion. Vertical motion was initiated and maintained by thrusting with both legs. Velocity control required two separate actions. When a foot was in the air, the leg swung forward a distance determined by the forward velocity. When a foot was on the ground, hip torques proportional to the error in forward velocity acted to stabilize velocity. Attitude control actively drove the body level by changing the difference in thrust between the two legs. The three parts acted separately and were coordinated by a sequencer.

Two variations of the control system were explored which resulted in two different gaits, trotting and bounding. The first gait, trotting, resulted when all three parts of the controller are used. The body stayed level as it bounced. The second gait, bounding, resulted when active attitude control was not used. The model stabilized in a mode of bouncing from one foot to another. Small accelerations could be accomplished without disturbing the bounding pattern. The model ran at speeds of $5 \mathrm{~m} / \mathrm{sec}$. The fact that the dog bounds 
with such a simple control system surprised us. We are currently trying to understand why the dog is stable in this gait. 


\subsection{Appendix. Simulation Parameters}

$\begin{array}{lll}\text { Symbol Description } & \text { Value }\end{array}$

Body:

$\begin{array}{ll}\mathrm{M}_{3} & \text { Mass } \\ \mathrm{I}_{3} & \text { Moment of Inertia } \\ \mathrm{r}_{3} & \text { Distance from Hip to CG }\end{array}$

Upper Link of Leg:

$\begin{array}{ll}\mathrm{M}_{2} & \text { Mass } \\ \mathrm{L}_{2} & \text { Moment of Inertia } \\ \mathrm{D} & \text { Length }\end{array}$

Lower Link of Leg:

$\begin{array}{ll}\mathrm{M}_{1} & \text { Mass } \\ \mathrm{I}_{1} & \text { Moment of Inertia } \\ \mathrm{D} & \text { Length }\end{array}$

Spring and Damper Constants:

$\begin{array}{ll}\mathrm{K}_{\mathrm{S}} & \text { Linear Leg Spring Constant } \\ \mathrm{K}_{\mathrm{G}} & \text { Ground Spring Constant } \\ \mathrm{B}_{\mathrm{G}} & \text { Ground Damper Constant }\end{array}$

Control Gains:

$\begin{array}{ll}\mathrm{K}_{1} & \text { Differential Thrust Proportional Gain } \\ \mathrm{K}_{2} & \text { Differential Thrust Differential Gain } \\ \mathrm{K}_{6} & \text { Velocity Error Hip Torque Gain } \\ \mathrm{K}_{\mathrm{p}} & \text { Hip Proportional Gain } \\ \mathrm{K}_{\mathrm{d}} & \text { Hip Differential Gain } \\ \mathrm{T} & \text { Constant Thrust for Vertical Control }\end{array}$

$30.0 \mathrm{~kg}$

$4.0 \mathrm{~kg} \mathrm{~m}^{2}$

$0.5 \mathrm{~m}$

$1.0 \mathrm{~kg}$ $0.008 \mathrm{~kg} \mathrm{~m}^{2}$

$0.3 \mathrm{~m}$

$0.25 \mathrm{~kg}$

$0.002 \mathrm{~kg} \mathrm{~m}^{2}$

$0.3 \mathrm{~m}$

$8.0 \mathrm{kN} / \mathrm{m}$

$50 \mathrm{kN} / \mathrm{m}$

$150 \mathrm{kN} \mathrm{s} / \mathrm{m}$

$2.0 \mathrm{~m} / \mathrm{rad}$

$0.08 \mathrm{~m} \mathrm{sec} / \mathrm{rad}$

$40.0 \mathrm{~N} \mathrm{~s}$

$150 \mathrm{~N} \mathrm{~m} / \mathrm{rad}$

$2 \mathrm{~N} \mathrm{~m} \mathrm{sec} / \mathrm{rad}$

$0.015 \mathrm{~m}$ 


\title{
4. Design and Construction of a Four-Legged Running Machine
}

\author{
H. Benjamin Brown, Jr. and Marc H. Raibert
}

\subsection{Introduction}

For several years our research on legged locomotion has focused on balance problems, while vigorously avoiding the coordination problems that are faced by systems with more than one leg. We have begun to address the problem of coordinating the actions of many legs through analysis and simulations, as described in Chapters 3, 5, and 7 of this report. It is now time to do some experiments.

For this purpose we have designed a small running machine with four legs. Figure 4-1 is a sketch showing the general configuration. The machine will be roughly the size and shape of a large dog. Unlike previous quadruped designs (Mosher, 1968; Frank, 1968; Hirose and Umetani, 1980), we intend for this machine to be fully dynamic. It will actively balance itself and employ ballistic flight phases, like those observed in animals.

With this experimental apparatus we plan to take ideas originally formulated in the context of systems with one leg, and to generalize and extend them to systems with several legs. Our intention is to show how gaits like the trot, the gallop, and the bound, and perhaps entirely new gaits that have not yet been discovered by natural systems, can be understood in terms of the simple concepts that emerged with the one-legged systems. Of course, new ideas that were not revealed in the one-legged work are also likely to emerge.

\subsection{Design Goals}

Five major decisions were made early to guide the design:

- The machine will have four legs.

- The machine will have a preferred direction of travel.

- The machine will use hydraulic power with proportional servo valves to power the hip motions and leg thrust.

- The machine will not carry its own power supplies nor its own computing.

- The machine will reuse as much design from the 3D one-legged machine as possible.

The decision to build a system with four legs, rather than two or three is difficult to justify. One reason for going to four legs is that the machine will provide a very rich set of behaviors. We believe that biped running is easily understood in terms of the one-leg results. But a machine with four legs offers the possibility of studying double, triple, and quadruple support phases, as components of running. 
FRONT/REAR VIEW

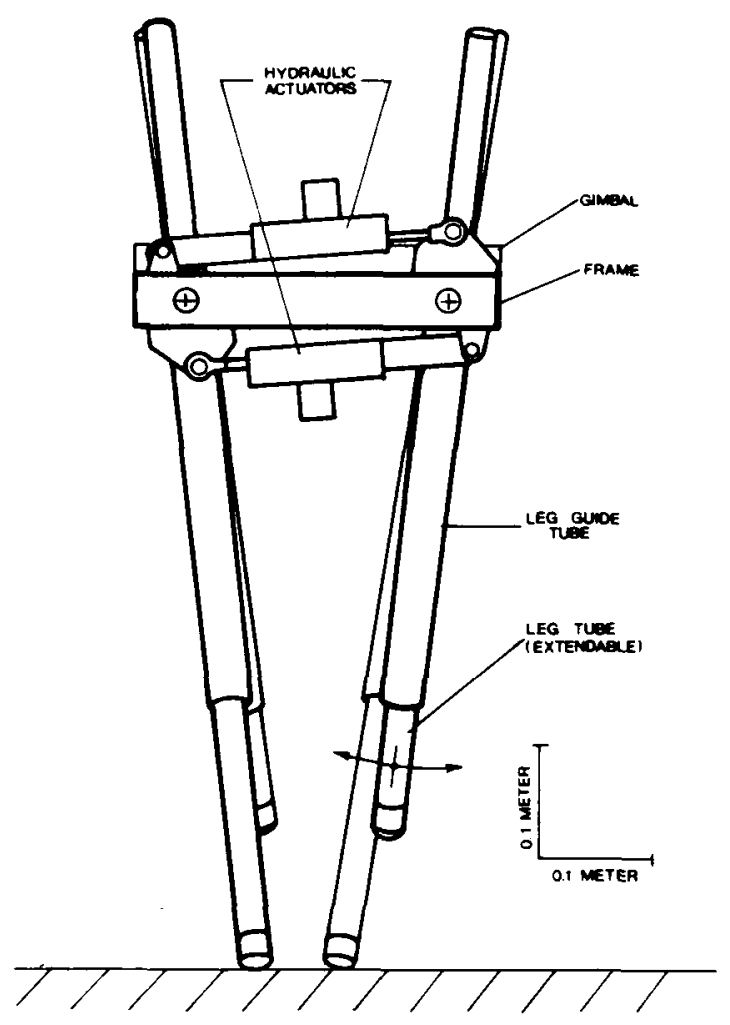

Figure 4-1: Diagram of four-legged running machine that is under construction. Like our previous designs, the machine will carry neither its power supply nor computing. The legs are $0.6 \mathrm{~m}$ long. The lateral hip separation is $0.24 \mathrm{~m}$, and the longitudinal separation is $0.8 \mathrm{~m}$. The machine will weigh about $35 \mathrm{Kg}$. Front and rear view above. Side view oppocite.

related to the number of legs. The weight overhead of the body is shared among the legs. For instance, even though the four-legged machine we are building has four times as many legs as the $3 \mathrm{D}$ one-legged machine, and each leg is heavier, the four legged machine will weigh only about twice as much. We believe that most useful legged vehicles of the future will have at least four legs.

A narrow body is a primary attraction of legged systems that balance. In order to have a narrow body, the legs must be placed near each other. However, in order to run fast, there must be a clear place for the legs to swing in the direction of travel, without hitting other legs. These two requirements are satisfied by placing the legs in pairs at opposite ends of the body. This results in a long body that can move fast in one direction, with limited travel in the other. Of course, other arrangements of legs are possible. For instance, it is not clear what would be wrong with putting all the legs in one long row. 


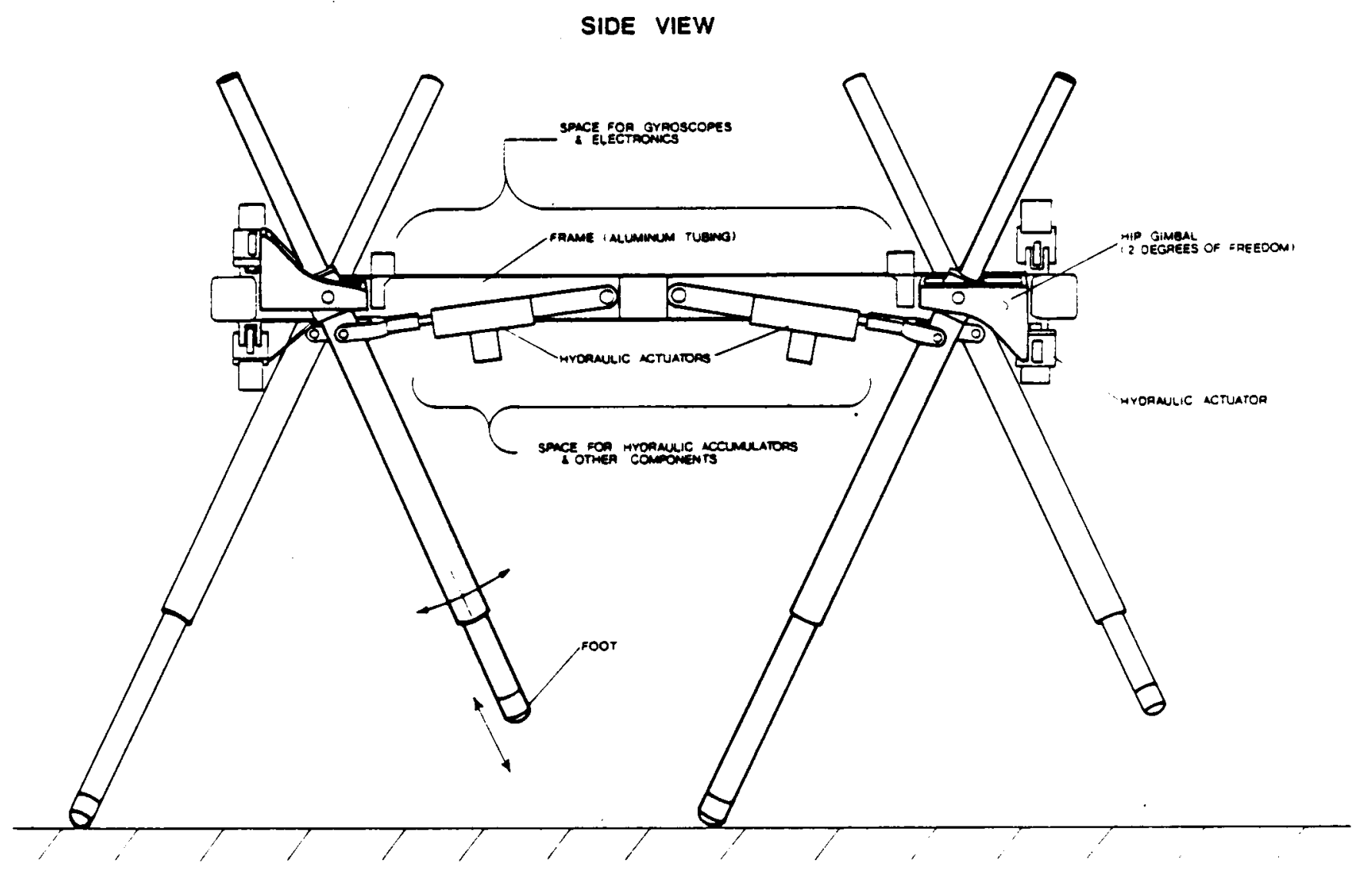

For the one-legged systems we have studied, it was possible to permit the bouncing motion of the body on the leg to proceed naturally, without being too concerned with the fine details of the motion. Since only one foot touched the ground at a time, the direction of the ground force was not influenced by the detailed pattern of leg thrust, and the leg thrust did not directly affect the attitude of the body. Therefore. on-off valves and a pneumatic actuator were adequate for control.

In multi-legged systems, legs will sometimes act jointly to deliver thrust and torque on the body. It will aiso be necessary to shorten the leg rapidly during recovery, in order to avoid stubbing the foot. In order to provide a more controllable thrust we designed a leg that thrusts with a hydraulic servo actuator. It retains a pneumatic spring for storage of bouncing energy. 


\subsection{Leg Design}

Most of the work that went into the design of the four-legged machine went into the design of the legs. The requirements for a leg to be used in a system with more than one leg are different from those to be used in systems with just one leg. The most important difference is that the leg of a one-legged system need not reuact during flight in order to swing forward for recovery. Since there is only one leg, it is recovered at the peak of the hop when there is plenty of clearance. In a multi-legged system it will be desirable to recover legs while other legs provide support. This means that the legs must shorten substantially to recover without hitting the ground. We also want to do more work on leaping over obstacles. To clear an obstacle it is desirable to shorten the legs during flight and then have time to lengthen them again before landing. This requires rapidly controlled leg length.

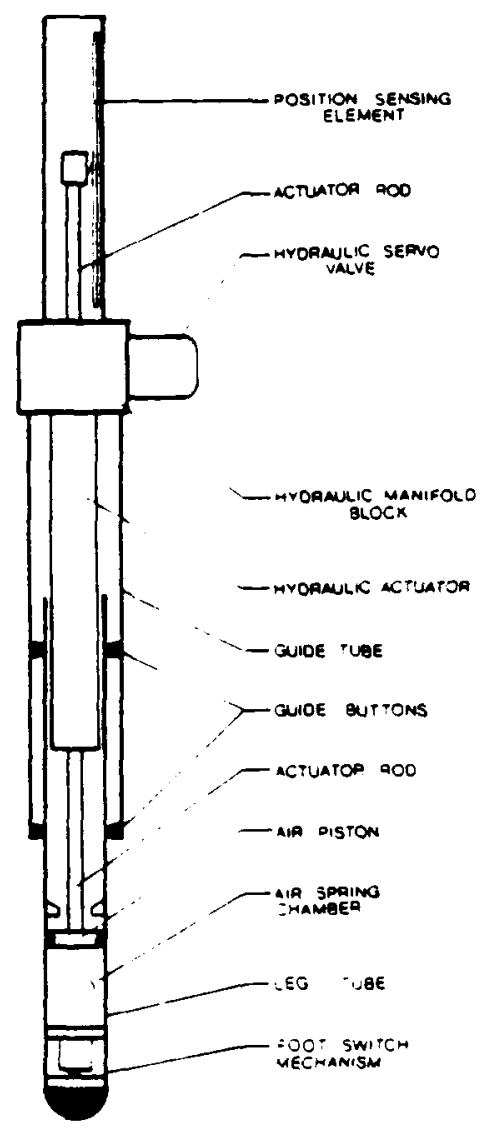

Figure 4-2: Schematic diagram of leg to be used in four-legged machine. It consists of a serro controlled hydraulic actuator in series with an air spring.

The four-legged machine will use its legs jointly in pairs. threes and perhaps all four, to simultancously push upward and forward on the body. In order for that to work. the concrol system must be abie wo control the precise force delivered by the extension motion of the legs. The simple air cylinder leg used in uter previous designs would not be adcquate for the four-legged machine. In particular, it cannot shorten and lenguhen 
rapidly, it requires large control valves, it uses compressed air very inefficiently, and it does not provide precise control of axial thrust. On the other hand, the air spring concept worked well, and we wanted to retain this feature.

We established a set of specifications to guide design of a new leg:

- The leg would be about $0.6 \mathrm{~m}$ long, from foot to hip.

- The leg must be able to change its length by about $0.2 \mathrm{~m}$,

- The leg must lengthen fast enough under load to produce a $0.2 \mathrm{~m}$ jump,

- The leg must generate an axial thrust of about $80 \mathrm{~kg}$,

- The unsprung mass should be minimized.

We chose a configuration with a linear hydraulic actuator in series with a passive air spring. We put the air spring near the foot to minimize the unsprung mass.

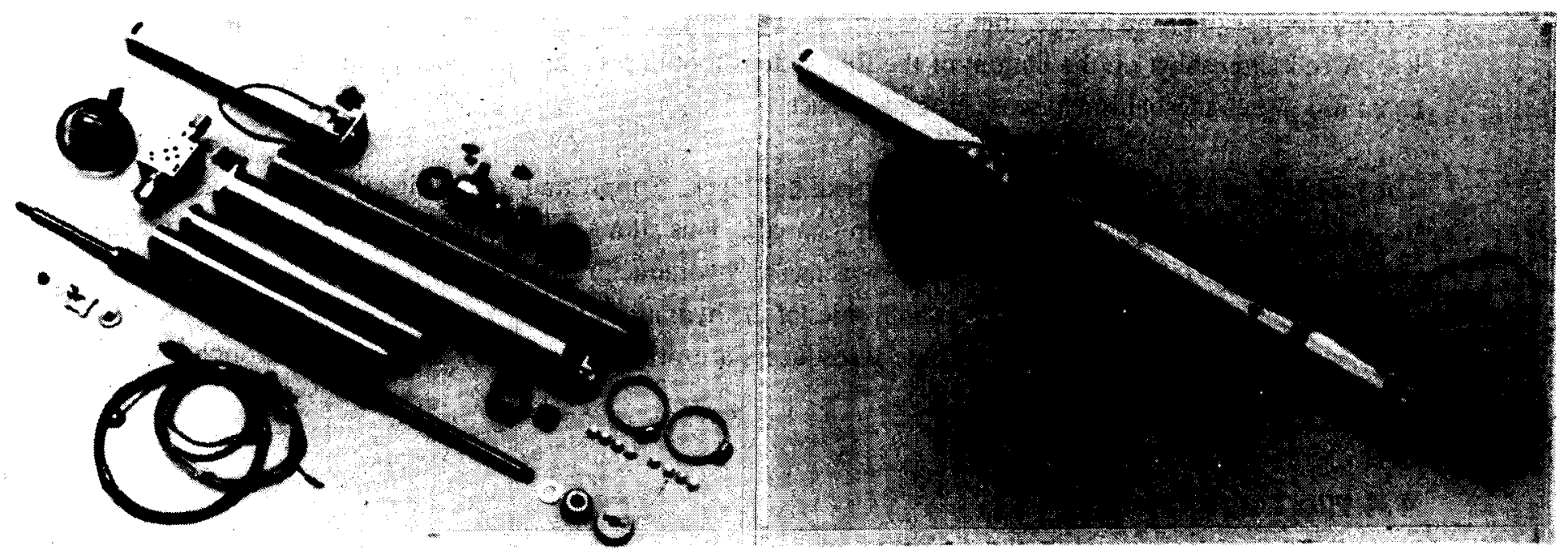

Figure 4-3: Prototype leg to be used in dynamic quadruped. Photographs show leg assembled and taken apart. The prototype was tested in the 3D one-legged machine.

The basic idea of the leg design is to take advantage of the high servo performance of a hydraulic system, and the compliant nature of a pneumatic system. See Fig. 4-2. The hydraulic actuator serves to determine the free position of the pneumatic spring. The hydraulic system permits the leg to change length quickly, and to deliver substantial force. The pneumatic system will permit the leg to store kinetic bouncing energy with good efficiency, while the hydraulic system should permit precise positioning during stance to control the forces delivered by each foot.

Details of the leg design are given in Figs. 4-3 and 4-4. The design consists of a set of concentric aluminum 
tubes of various diameters and lengths. A $3 / 8$ inch diameter steel actuator rod at the centerline is driven by a $1 / 2$ inch diameter piston, bonded to the rod, inside a hard-coated aluminum cylinder. The lower end of the rod attaches to a second piston, 1-1/8 inch diameter, that forms the upper enclosure of the air spring. The upper end of the rod drives a wiper along a linear position-sensing element.

Two concentric tubes outside the cylinder tube provide oil flow to and from the lower end of the piston and lower case drain. The upper ends of all three cylinders connect to a manifold block, that has passages for supply and return oil, and for actuation oil from the valve. It also has mounting ports for the servo valve and pressure transducers. To minimize friction, high-pressure hydraulic seals are formed by the annular clearances at the piston and rod bushings. A case drain and O-ring seal, outboard of each rod bushing, prevent external leakage.

Outside the hydraulic actuators are two additional tubes. The inner of these, the leg tube, attaches to the foot and forms the cylinder portion of the air spring. The outer tube mounts to the manifold block, and has guide buttons that constrain the leg tube to move linearly. The guide tube also carries an alignment block that prevents leg twisting, and a wiper that slides on a linear position sensing element attached to the outside of the leg. A rubber cushion on the bottom of the foot softens foot impact, and provides good traction. The foot contains a switch mechanism to sense ground contact.

The hip inner pivot is an integral part of the manifold block. Supply and return oil will be fed to and from the manifold block through passages in the hip gimbals. This allows the hip joint to be kept quite compact, and minimizes flexing of hoses. There are provisions for hydraulic pressure transducers to be mounted in the manifold block, to measure pressures on both sides of the hydraulic piston. A transducer may also be used to measure pressure in the air spring. These transducers are primarily for data acquisition, although use in control is possible.

\subsection{Hip Design}

The second major consideration in the design of the machine was the choice of actuators to drive the hip. Based on experience with the 3D hopper, and analyses performed previously during the design of that machine, only hydraulic actuation was considered. Rotary hydraulic actuators would have been appropriate for at least one axis of hip rotation. However we decided to use the linear actuators that we had designed for the one-legged machine, rather than to delay the project while we designed and tested a new actuator.

We could not find a reasonable alternative to a two-axis gimbal arrangement for the hip. We decided to use anti-friction ball bearings, rather than sleeve bearings, to minimize friction and free play in the joints. We wanted the leg to swing more than $\pm 30^{\circ}$ in the fore and aft directions.

The machine clearly has a preferred direction of travel, so the $\mathrm{X}$ and $\mathrm{Y}$ bchavior of the leg did not necd to be the same. We decided to keep the machine symmetrical left-to-right, and to have no skew in the fore and aft 


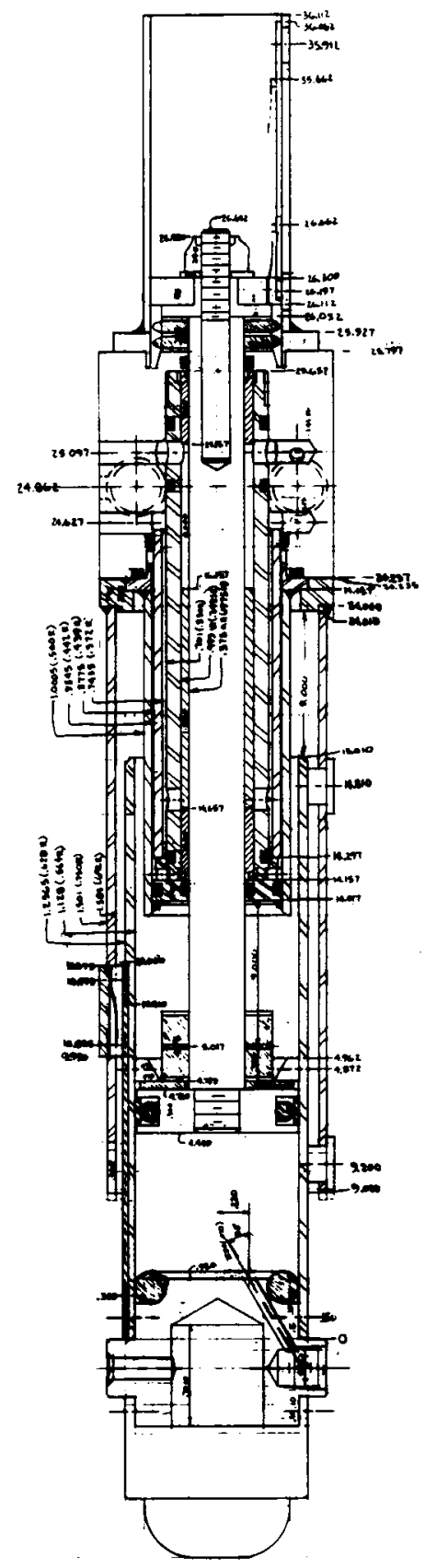

Figure 4-4: Details of leg design. 
leg motion. Therefore, there is no lateral asymmetry of the fore/aft leg motion. We also wanted to keep the machine as narrow as possible, both overall and at the hip centers, to enhance the forward mobility of the machine. The arrangement shown in Fig. 4-1 provides attachments for the fore/aft actuators directly to the leg below the hip joint. The transverse actuators drive the leg through the gimbal. This arrangement permits the transverse actuators to be overlapped as shown, with no significant deviation from lateral symmetrical performance. This configuration results in a minimum overall width of approximately $0.33 \mathrm{~m}$, and an overall length of about $1.0 \mathrm{~m}$. The hips are spaced closely enough so that the feet can reach either side of the machine's centerline.

The frame was designed to give good torsional stiffness and strength, to provide mounting areas and protection for on-board components, and to provide convenient places to hold onto the machine during experiments. The equipment mounted on the frame includes two hydraulic accumulators, hydraulic distribution manifolds, two gyroscopes, and electronic interface boards. With twelve hydraulic actuators, hose routing and manifolding must be carefully planned to minimize hydraulic leaks, to make repair possible, and to look nice. The electronic interface boards will be similar to those used on the 3D hopping machine.

At the time of this writing, construction of the machine is well under way. We expect the machine to be assembled by February 1984, and to see it running in late Spring. 


\title{
5. Is Gait a Coupled Oscillation?
}

\author{
Jeffrey Koechling
}

\subsection{Abstract}

We explore the idea that gait is determined by coupled mechanical oscillators. Our model has two springy legs attached to a rigid body. We analyze three modes of oscillation of the model: bouncing, rocking, and swaying. Computer simulations of the model show both the bouncing and the rocking modes. By tuning the model to adjust the relative amplitude and frequency of these modes, we hope to show changes in the motion of the model that are analogous to gait transitions in animals.

\subsection{Introduction}

Multi-legged animals use different gaits to move at different speeds. For example, a cat will use a walk below one mile per hour, switch to a trot around two miles an hour, and gallop at over ten miles an hour (Pearson, 1976). This sequence of gaits is typical for four legged animals.

We want to understand the mechanism that determines what gaits are used and at what speeds gait transitions occur. Our working hypothesis is that the mechanical system determines the gaits and gait transitions observed in animals. The legs and bodies of animals are composed of tissue having mass, stiffness and viscosity. This tissue forms coupled mechanical oscillators with natural modes of oscillation. These natural modes of uscillation correspond to the gaits that the animal uses.

We have modeled a planar system with two legs connected to a rigid body. We want to "pluck" the system in different ways and see it exhibit different motions. We would like to show that the mode of oscillation of the model depend on its initial state. By varying the parameters of the model, we can see how varying the stiffness of the legs alters the natural mades of the model. The idea is to demonstrate that a system with characteristics similar to those found in nature can change from one pattern of motion to another, without being explicitly forced to do so.

We have implemented a simulation of the model and made it run. The stiffness of the lcgs and the stiffness of the hips determine the phase and amplitude relationships between the bouncing and rocking modes of oscillation. By varying these relationships, we can affect the stability of the model and the pattern of motion of the legs. We have not yet achieved a clear cut gait transition, but we are making progress toward that goal.

In section 5.3 we briefly review some ideas about coupled oscillators in the control of locomotion. In section 5.4 we describe the model, and in section 5.5 we analyze the oscillations of the system. In section 5.6 we present some data from both stable and unstable simulations. Finally, in Section 5.7 we discuss the meaning of these preliminary results and some ideas for further work. 


\subsection{Background}

We do not know how gaits are chosen, nor do we know the source of the pattern of motion that characterizes each gait. Here are some of the possibilities:

1. There are neurons in the central nervous system that generate a pattern of nerve impulses. The limbs are driven in this basic pattern, subject to mechanical limitations. The gait changes when this neural pattern generator begins producing a different pattern (Wilson, 1966; Wetzel et al., 1975).

2. There are several control systems that interact to produce the characteristic motions of the gait, much like the controllers used in our laboratory machines. These controllers can be open loop systems, like the pattern generator mentioned in 1 above, or closed loop systems with sensors in the limbs and feedback paths through the central nervous system. A gait change results from a change in the phase relationships among the controllers. This is a case of neural coupled oscillators (Pearson, 1976).

3. The animal's bones, muscles and tendons form a mechanical system. This system has certain characteristic modes and frequencies of oscillation that depend on the mass, stiffness, and viscosity of the tissue. Each of the natural modes of oscillation corresponds to a different gait, and is best suited for moving at one speed. When the animal tries to change speed by forcing its legs and body to oscillate at a different frequency, the system moves less efficiently. When the motion approaches a frequency that is characteristic of a different natural mode, the pattern of motion changes to that gait. This is a case of mechanical coupled oscillators, as opposed to the neural coupled oscillators in 1 above (Alexander, 1974; Alexander and Vernon, 1975).

4. The mechanical system has natural modes of oscillation, as in 3 above, but the nervous system controls the mechanical properties of the muscles. Altering the stiffness and viscosity of the muscles changes the natural modes of oscillation. Rather than force the system to go faster or slower until it switches to a different gait, the nervous system adjusts the mechanical system to oscillate with a gait and frequency that results in the desired speed of locomotion.

The idea that coupled oscillators control gait is not new. Much of the previous work that we are aware of has been done by neurophysiologists, and concentrates on spinal oscillators, as in 1 above, or on peripheral-spinal oscillators, as in 2 above. We are investigating the last two possibilities, 3 and 4 above.

\subsection{The Model}

The model is planar, free to translate vertically and horizontally, and to rotate in the plane. Two springy legs are attached to the body by springy hips. There are five rigid links altogether, each of which has mass and moment of inertia.

The legs are not articulated like biological legs. Instead they have a sliding "knee", like a telescope. This type of leg has different dynamic properties from an articulated leg. Kinematically, it performs the same function. Both types of legs change the distance and direction of the foot from the hip. We find telescoping legs to be very effective on physical machines that we experiment with in the laboratory. 


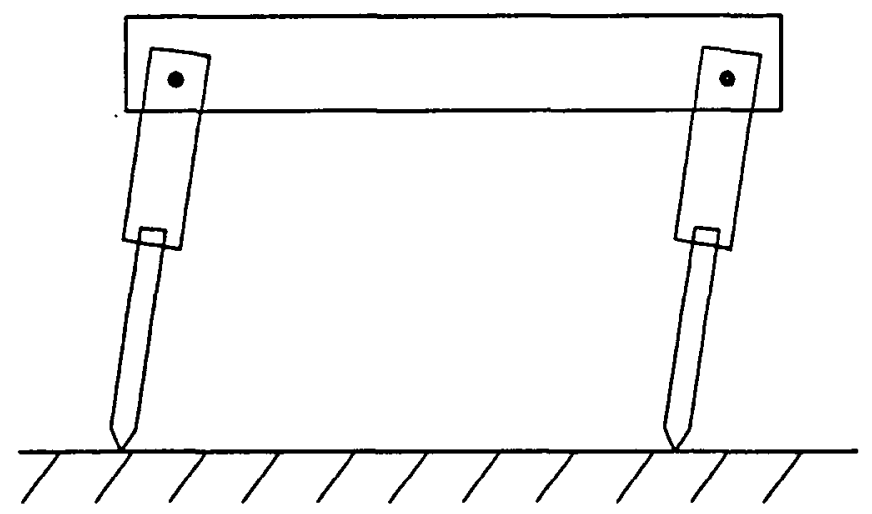

Figure 5-1: The model has five rigid links and four springy joints. The length of the legs can change, as can the angle of the legs with respect to the body. The links all have mass and moment of inertia

Between the lower part and the upper part of each leg there is a spring and a damper. These springs and dampers apply forces that lengthen or shorten the leg. Between the upper part of each leg and the body, there is another spring and damper. These apply torques that change the angle between the leg and the body. The springs and dampers can be thought of as analogs to the groups of muscles that apply forces to biological legs. A muscle can be modeled as a spring, a damper and an active force generator. Fig. 5-2 shows the springs, masses and dampers symbolically.

In a physical machine or organism there are kinematic constraints that restrict the range of motion of each joint. In this model, there are stops that impose a maximum and minimum length on the legs. When the leg reaches the maximum or minimum length, it hits a stop. The stops are very stiff springs in parallel with dampers. There are no stops to restrict the angle of the leg with respect to the body.

Legged locomotion requires interaction with the ground. Reaction forces occur whenever the feet are in contact with the ground. We have used a stiff spring and a damper to represent the combined compliance of the ground and the foot. When a foot touches the ground, the spring and damper in the ground become active, pushing back on the foot. The spring applies forces tending to drive the foot to the point of initial contact with the ground. If the foot is slightly below the surface and moving toward the surface, this model of the ground can produce small negative vertical reaction forces. This happens when the damper force away from the surface exceeds the spring force toward the surface. The result is a net force that tends to hold the foot onto the ground. This "sticky ground" is unrealistic, so any negative vertical component of the reaction force is set to zero.

The principal motion of the model is a cyclic vertical bouncing. When the model is not on the ground, it is pulled downward by gravity. The feet hit the ground, and the legs compress, slowing the descent and accelerating the body upward. The model leaves the ground, and its ascent is slowed by gravity. This cycle 


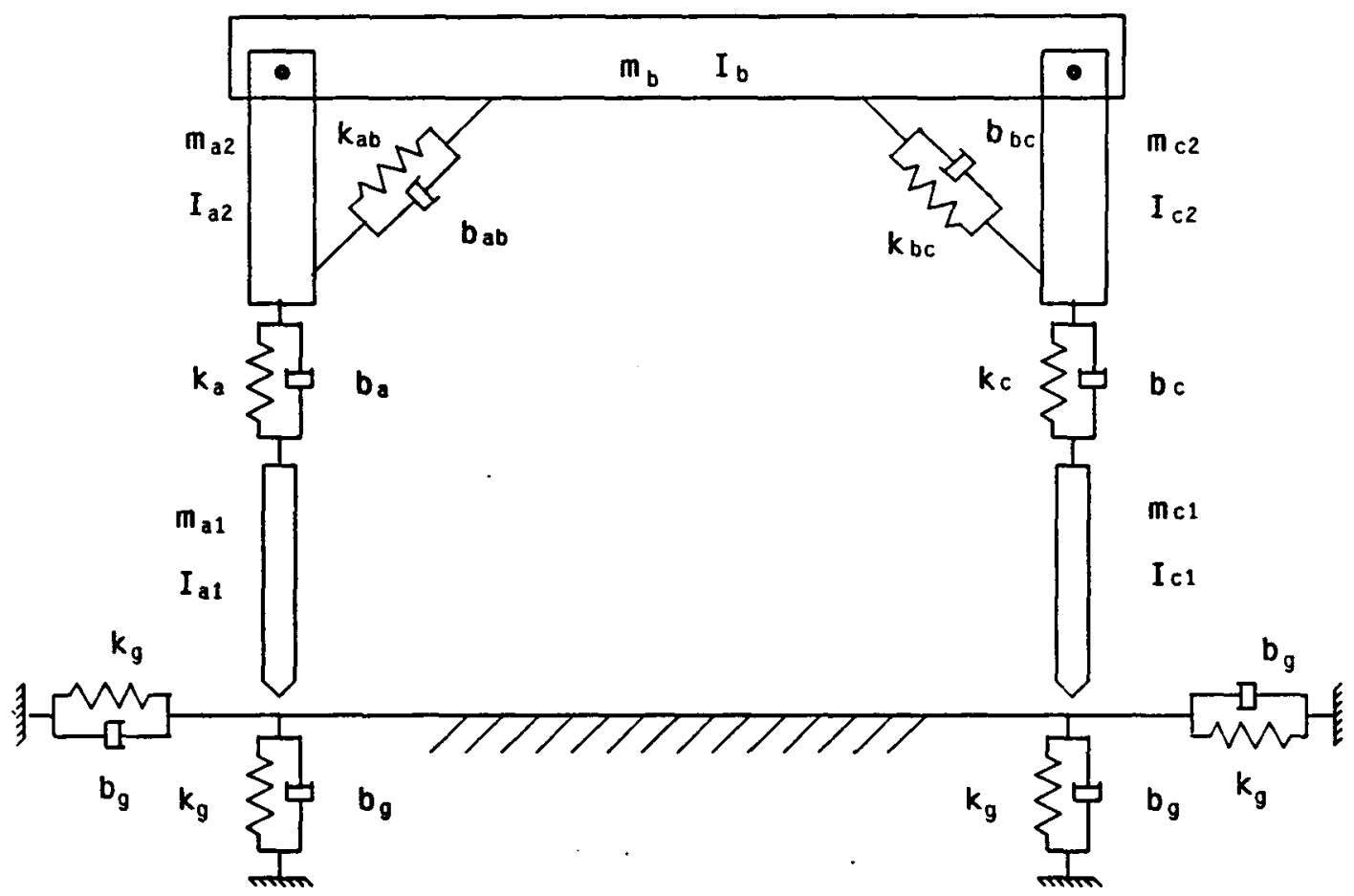

Figure 5-2: This figure shows the model as a collection of springs, masses, and dampers. The springs and dampers at the hips produce torques that are proportional to the angle and angular velocity of the hip.

repeats as long as the legs remain under the body and there is enough total energy for the model to leave the ground. The vertical bouncing cycle is described in detail on section 5.5.

During the vertical bouncing cycle, the model dissipates energy. There are losses in the dampers, and impacts with the ground, and with the stops. When an impact occurs momentum is conserved and energy is lost. In order to operate in a steady state, we add energy to the model. When a leg reaches its minimum length and begins to lengthen, we increase its equilibrium length. The effect is the same as having an actuator compress the spring, adding potential energy to the system. We only add energy to a leg when the foot is on the ground. Once the foot leaves the ground we return the equilibrium length of the leg to the original value.

In Appendix A we develop the equations of motion for the model and outline the way that we solve them. Appendix $B$ is a list of the parameter values used for the simulations in this report. 


\subsection{Modes of Oscillation}

The model described in Section 5.4 has three modes of oscillation, as shown in Fig. 5-3. Vertical bouncing, where the legs remain vertical and the body remains horizontal, dominates the motion of the system. When both feet are on the ground there is a see-saw rocking mode, in which the legs remain vertical, but one is getting shorter while the other is getting longer. In this mode, the body rocks like a see-saw. Finally there is a swaying motion where the system resembles an inverted pendulum. In this mode the body remains level while the legs pivot about the feet.
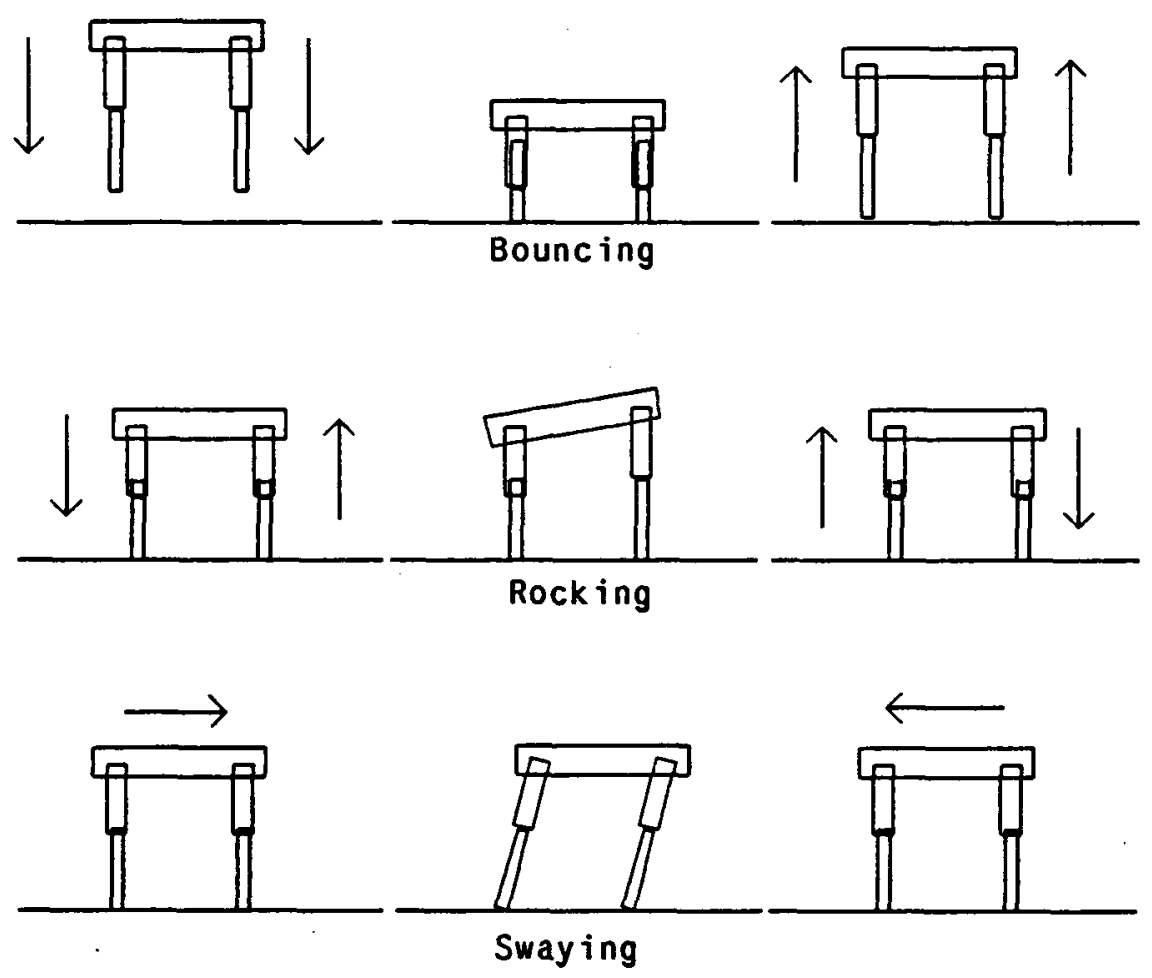

Figure 5-3: These are the modes of oscillation of the model.

In this section, we make some simplifications and then analyze each mode. One simplification is that we look only at the cases where either both of the feet are on the ground or neither of the feet are on the ground. When both feet are on the ground, we consider the model to be a linear second order oscillator. We look most carefully at bouncing, solving the equations of motion for a complete cycle. For the rocking and swaying modes, we find the natural frequency and the damping ratio. 


\subsubsection{Vertical Bouncing}

We need to define some terms to talk about the vertical motion. When the feet are on the ground, the model is in the stance phase, otherwise it is in the flight phase. The transition from flight to stance is called touchdown, the transition from stance to flight is called lift-off. The lowest point in the cycle, bottom, occurs during stance. The highest point in the cycle, top, occurs during flight. See Fig. 5-4.

When the body is level and bouncing straight up and down, the model alternates between harmonic motion during stance and ballistic motion during flight. From touchdown though lift-off the motion is that of a damped spring-mass oscillator. The motion changes slightly when the model reaches bottom and begins to accelerate upward. This is because we change the equilibrium length of the legs to add energy to the system: From touchdown to bottom the vertical motion is described by the second order differential equation:

$$
-m \ddot{y}-2 b \dot{y}-2 k\left(y-y_{0}\right)-m g=0
$$

where

$\mathrm{m} \quad$ is the sprung mass of the model

(Everything except for the lower legs),

b is the damping coefficient of the legs,

$k$ is the spring constant of the legs,

$y$ is the body height,

$y_{0} \quad$ is the rest length of the legs.

Solving (5.1) gives the following expressions for the height and vertical velocity:

$$
\begin{aligned}
& y=y_{0}-\frac{g}{\omega_{n}^{2}}+e^{-\zeta \omega_{n}\left(t-t_{t d}\right)}\left\{A \sin \left[\omega_{d}\left(t-t_{t d}\right)\right]+B \cos \left[\omega_{d}\left(t-t_{t d}\right)\right]\right\} \\
& y=e^{-\zeta \omega_{n}\left(t-t_{t d}\right)}\left\{C \sin \left[\omega_{d}\left(t-t_{i d}\right)\right]+D \cos \left[\omega_{d}\left(t-t_{t d}\right)\right]\right\} \\
& \text { where }
\end{aligned}
$$

$$
\begin{aligned}
& A=\left(\frac{1}{\omega_{d}}\right)\left[\left(\dot{y}_{t d}+\zeta \omega_{n}\left(y_{t d}-y_{0}+\frac{g}{\omega_{n}^{2}}\right)\right]\right. \\
& B=\left(y_{t d}-y_{0}+\frac{g}{\omega_{n}^{2}}\right) \\
& C=\left(\frac{-1}{\omega_{d}}\right)\left[\zeta \omega_{n}\left(\dot{y}_{t d}\right)+\omega_{n}^{2}\left(y_{t d}-y_{0}+\frac{g}{\omega_{n}^{2}}\right)\right] \\
& D=\dot{y}_{t d}
\end{aligned}
$$

$t_{\mathrm{td}}$ is the time when touchdown happens, 


$$
\begin{aligned}
& \underline{y}_{\mathrm{td}} \text { is the height of the body, } \\
& \dot{y}_{\mathrm{td}} \text { is the vertical velocity at touchdown, and } \\
& \omega_{\mathrm{n}}=\sqrt{\frac{2 \mathrm{k}}{\mathrm{m}}}, \zeta=\frac{2 \mathrm{~b}}{2 \sqrt{2 \mathrm{~km}}}, \text { and } \omega_{\mathrm{d}}=\omega_{\mathrm{n}} \sqrt{1-\zeta^{2}} .
\end{aligned}
$$

When the model reaches bottom the velocity is zero. The time to reach bottom, $t_{b}$, is obtained by setting (5.3) equal to zero.

$$
t_{b}=t_{t d}+\left(\frac{1}{\omega_{d}}\right) \operatorname{atan}\left(\frac{\omega_{d} \dot{y}_{t d}}{\zeta \omega_{d} \dot{y}_{t d}+\omega_{n}^{2}\left(y_{t d}-y_{0}\right)+g}\right)
$$

At $c_{b}$, the rest length of the legs changes from $y_{0}$ to $y_{0}^{*}$. The equations of motion for the remainder of the stance phase are:

$$
\begin{aligned}
& y=y_{0}^{*}-\frac{g}{\omega_{n}^{2}}+e^{-5 \omega_{n}\left(t-t_{b}\right)}\left\{E \sin \left[\omega_{d}\left(t-t_{b}\right)\right]+F \cos \left[\omega_{d}\left(t-t_{b}\right)\right]\right\} \\
& y=e^{-5 \omega_{n}\left(t-t_{b}\right)}\left\{G \sin \left[\omega_{d}\left(t-t_{b}\right)\right]\right\}
\end{aligned}
$$

where

$$
\begin{aligned}
& E=\frac{\zeta \omega_{n}}{\omega_{d}}\left(y_{b}-y_{0}^{*}+\frac{g}{\omega_{n}^{2}}\right) \\
& F=\left(y_{b}-y_{0}^{*}+\frac{g}{\omega_{n}^{2}}\right) \\
& G=\frac{-\omega_{n}^{2}}{\omega_{d}}\left(y_{b}-y_{0}^{*}+\frac{g}{\omega_{n}^{2}}\right)
\end{aligned}
$$

Lift-off and touchdown both occur with the legs at maximum length. When the legs extend to $y_{\text {td }}$ the stops that establish the maximum leg length hit the lower leg and accelerate it upward. Momentum is conserved during the impact, and energy is dissipated. The vertical velocity of the body decreases by the ratio between the sprung mass of the model and the total mass. The sudden reduction in velocity can be seen as a notch on the phase plot in Fig. 5-4. Setting (5.10) equal to $y_{t d}$ gives a transcendental expression for the time of lift-off, to.

$$
y_{t d}=y_{0}^{*}-\frac{g}{\omega_{n}^{2}}+e^{-\zeta \omega_{n}\left(t_{0}-t_{b}\right)}\left\{E \sin \left[\omega_{d}\left(t_{10}-t_{b}\right)\right]+F \cos \left[\omega_{d}\left(t_{10}-t_{b}\right)\right]\right\}
$$


where $E$ and $F$ are given by (5.12) and (5.13).

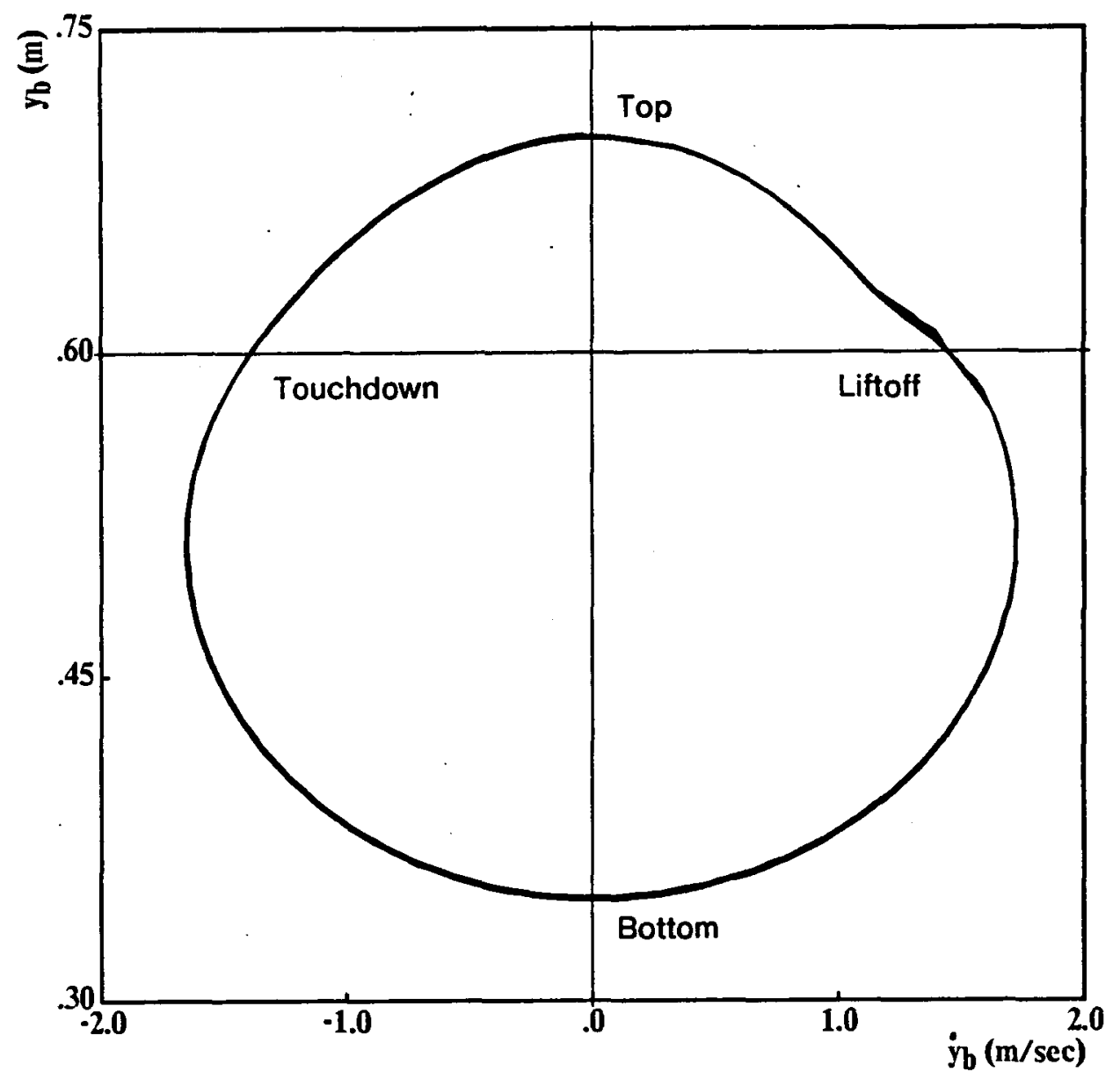

Figure 5-4: The vertical position of the center of gravity of the body is plotted along the vertical axis, and the vertical velocity along the horizontal axis. The upper part of the plot is parabolic, the motion of a body experiencing the constant acceleration of gravity. The lower part is harmonic, the motion of a spring mass oscillator. Note the notch caused by the impact of the stops against the lower legs. This phase plot is from simulation r.319.8 and shows several cycles of vertical bouncing. The model parameters are listed in Appendix B.

Once lift-off has occurred, the vertical motion is described by:

$$
-M \ddot{y}-M g=0
$$

where $M$ is the total mass of the system.

Solving (5.16) gives: 


$$
\begin{aligned}
& y=y_{10}=\frac{g\left(t-t_{10}\right)^{2}}{2} \\
& \dot{y}=\dot{y}_{10}-g\left(t-t_{10}\right)
\end{aligned}
$$

In Table 5-1 the vertical motions predicted by this analysis are compared with those measured from a simulation. Most of the values differ by less than $3 \%$. The velocity just after lift-off, $\dot{\mathrm{y}}_{\mathrm{lo}}^{+}$, differs by $10 \%$. We attribute the discrepancy to differences in the modeling of the compliance of the ground and the stops. In the simulation these are modeled as stiff $(k=50000 \mathrm{~N} / \mathrm{m})$ springs, while in the analysis they are modeled as rigid

\begin{tabular}{|c|c|c|}
\hline Quantity & Analysis & Simulation \\
\hline Natural Frequency, $\omega_{n}$ & $10.260 \mathrm{rad} / \mathrm{sec}$ & \\
\hline Damping Ratio, $\zeta$ & 0.0256 & \\
\hline Damped Frequency, $\omega_{d}$ & $10.256 \mathrm{rad} / \mathrm{sec}$ & \\
\hline Velocity at Touchdown, $\dot{y}_{t d}$ & $-1.981 \mathrm{~m} / \mathrm{sec}$ & $-1.962 \mathrm{~m} / \mathrm{sec}$ \\
\hline Time at Touchdown, $t_{t d}$ & $0.202 \mathrm{sec}$ & $0.200 \mathrm{sec}$ \\
\hline Time at Bottom, $t_{b}$ & $0.397 \mathrm{sec}$ & $0.400 \mathrm{sec}$ \\
\hline Position at Bottom, $y_{b}$ & $0.305 \mathrm{~m}$ & $0.301 \mathrm{~m}$ \\
\hline Time at Lift-off, $t_{0}$ & $0.587 \mathrm{sec}$ & $0.600 \mathrm{sec}$ \\
\hline Velocity before Lift-off, $\dot{y}_{1}^{-}$ & $2.01 \mathrm{~m} / \mathrm{sec}$ & $2.03 \mathrm{~m} / \mathrm{sec}$ \\
\hline Velocity after Lift-off, $\dot{y}_{10}^{+10}$ & $1.901 \mathrm{~m} / \mathrm{sec}$ & $1.735 \mathrm{~m} / \mathrm{sec}$ \\
\hline Time at Touchdown, $\mathrm{t}_{\mathrm{td} 2}$ & 0.976 seconds & 0.980 seconds \\
\hline Period & 0.774 seconds & 0.780 seconds \\
\hline
\end{tabular}
constraints.

Figure 5-1: This table compares the vertical bouncing motion obtained from the numerical simulation with that predicted by the analysis in this section. The simulation data are from simulation r.319.1. The model was dropped from 0.2 meters with the body level. The parameters of the model are listed in Appendix B.

\subsubsection{Rocking}

When the feet are on the ground, the model forms a torsional oscillator. When the body is perturbed from the horizontal by an angle $\theta$, a restoring torque is generated by the hip springs and by the differential forces in the leg springs. Using the small angle approximation, the equation of motion is: 
$-\mathrm{I} \ddot{\theta}-\left(2 \mathrm{~B}+\mathrm{bs} \mathrm{s}^{2}\right) \dot{\theta}-\left(2 \mathrm{~K}+\mathrm{ks}^{2}\right) \theta=0$

where

$$
\begin{aligned}
& \mathrm{I}=\mathrm{I}_{\mathrm{b}}+\frac{\mathrm{s}^{2} \mathrm{~m}_{\text {upperleg }}}{2} \\
& \mathrm{~S} \text { is the distance from the body center of gravity to the hips, } \\
& \mathrm{B} \text { is the damping coefficient at the hip, and } \\
& \mathrm{K} \text { is the spring constant at the hip. }
\end{aligned}
$$

The oscillation will be characterized by:

$$
\omega_{\mathrm{n}}=\sqrt{\frac{2 \mathrm{~K}+\mathrm{kl} \mathrm{l}^{2}}{\mathrm{I}}}, \zeta=\frac{2 \mathrm{~B}+\mathrm{bl^{2 }}}{2 \sqrt{\mathrm{I}\left(2 \mathrm{~K}+\mathrm{kl}^{2}\right)}} \text {, and } \omega_{\mathrm{d}}=\omega_{\mathrm{n}} \sqrt{1-\zeta^{2}} \text {. }
$$

\subsubsection{Swaying}

In this mode of oscillation. the legs remain at a constant length while they pivot about the feet, and the body remains level. Again we use the sinall angle approximation, and the equation of motion is:

$$
-I \ddot{\theta}-2 \mathrm{~B} \dot{\theta}-\left(2 \mathrm{~K}-2 \mathrm{~m}_{\mathrm{L}} \mathrm{gr}-\mathrm{m}_{\mathrm{b}} \mathrm{gL}\right) \theta=0
$$

where

$$
\begin{aligned}
& I=2 I_{L}+2 m_{L} r^{2}+m_{b} L^{2}, \\
& L \quad \text { is the length of the legs, } \\
& r \quad \text { is the distance from the foot to the center of gravity of the legs, } \\
& m_{L}, I_{L} \text { are the mass and moment of inertia of the legs, and } \\
& m_{b} \quad \text { is the mass of the body. }
\end{aligned}
$$

The oscillation will be characterized by:

$$
\begin{aligned}
& \omega_{\mathrm{n}}=\sqrt{\frac{2 \mathrm{~K}-2 \mathrm{~m}_{\mathrm{L}} g r-m_{\mathrm{b}} g l}{I}}, \\
& \zeta=\frac{2 B}{2 \sqrt{\left(2 \mathrm{~K}-2 \mathrm{~m}_{\mathrm{L}} \mathrm{gr}-\mathrm{m}_{\mathrm{b}} g l\right)}}, \text { and } \\
& \omega_{d}=\omega_{\mathrm{n}} \sqrt{1-\zeta^{2}}
\end{aligned}
$$




\subsection{Results}

We show some of the state information from a simulation in Fig. 5-5. The model was dropped from a height of $0.2 \mathrm{~m}$ with the body level. This is a stable simulation: the motion is periodic and continues indefinitely.
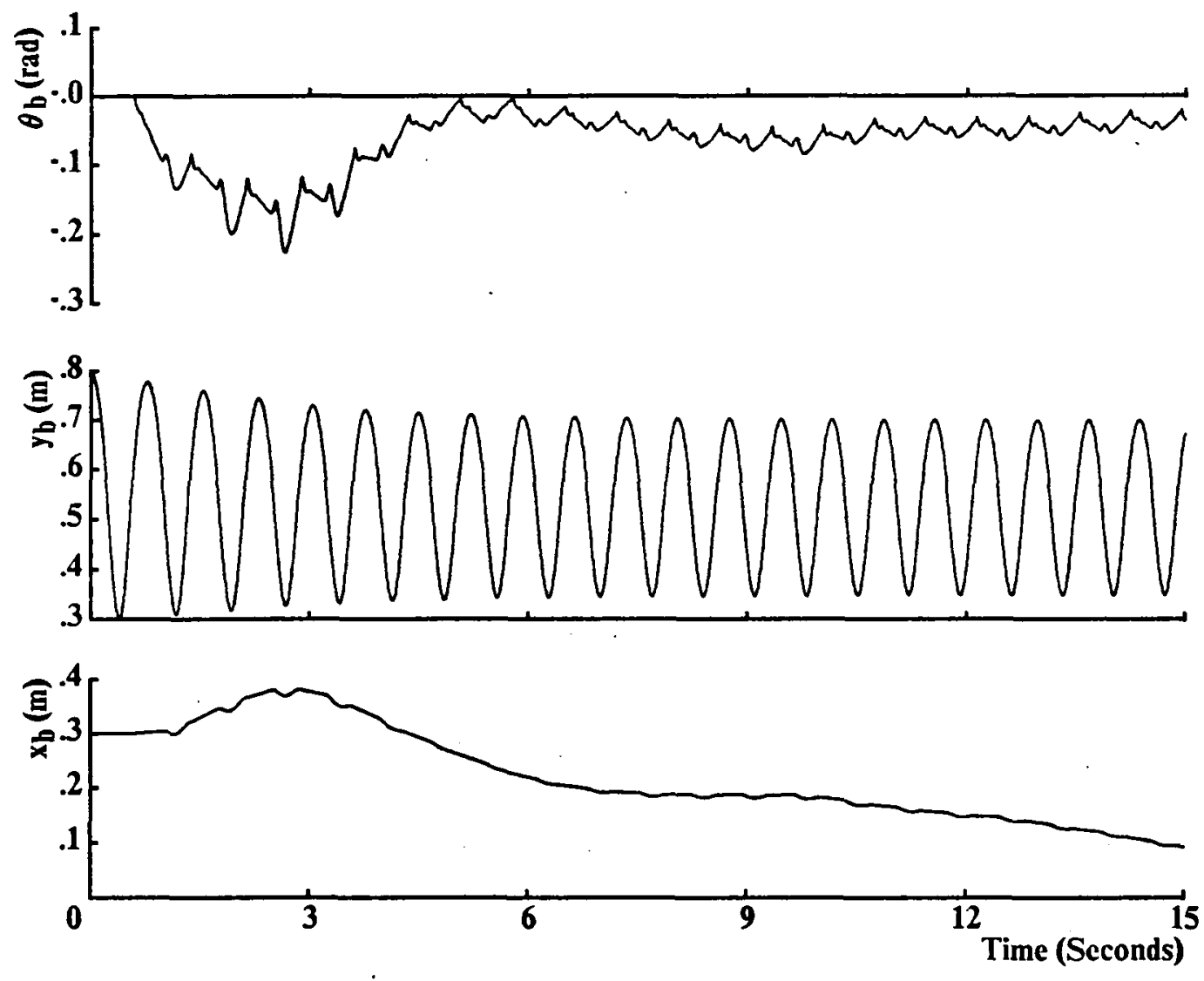

Figure 5-5: The plots show the angle of the body, and the horizontal and vertical displacement of the center of gravity of the body. The model was dropped from a height of $0.2 \mathrm{~m}$ with the body level. Note the two frequencies of oscillation: a high bouncing frequency and a lower rocking frequency that decays away. The data are from simulation r.319.8.

Two distinct modes of oscillation are visible. The "heartbeat" of the model is the vertical bouncing with a frequency of $1.28 \mathrm{~Hz}$. The amplitude of this mode decreases slightly from the initial height to a height where the energy losses in a hopping cycle match the energy addition in a cycle. The second mode is a $0.15 \mathrm{~Hz}$ rocking which decays as the simulation progresses. Fig. 5-6 shows the length of one leg and the hip angle for that leg with this frequency outlined.

Not all of our simulations are stable. Fig. 5-7 shows some of the state information for a simulation where the model fell over after a few seconds. We ran two sets of simulations to learn something about the limits of stability. Tables 5-2 and 5-3 show the results. Beginning with the model that behaved as shown in Fig. 5-5, 

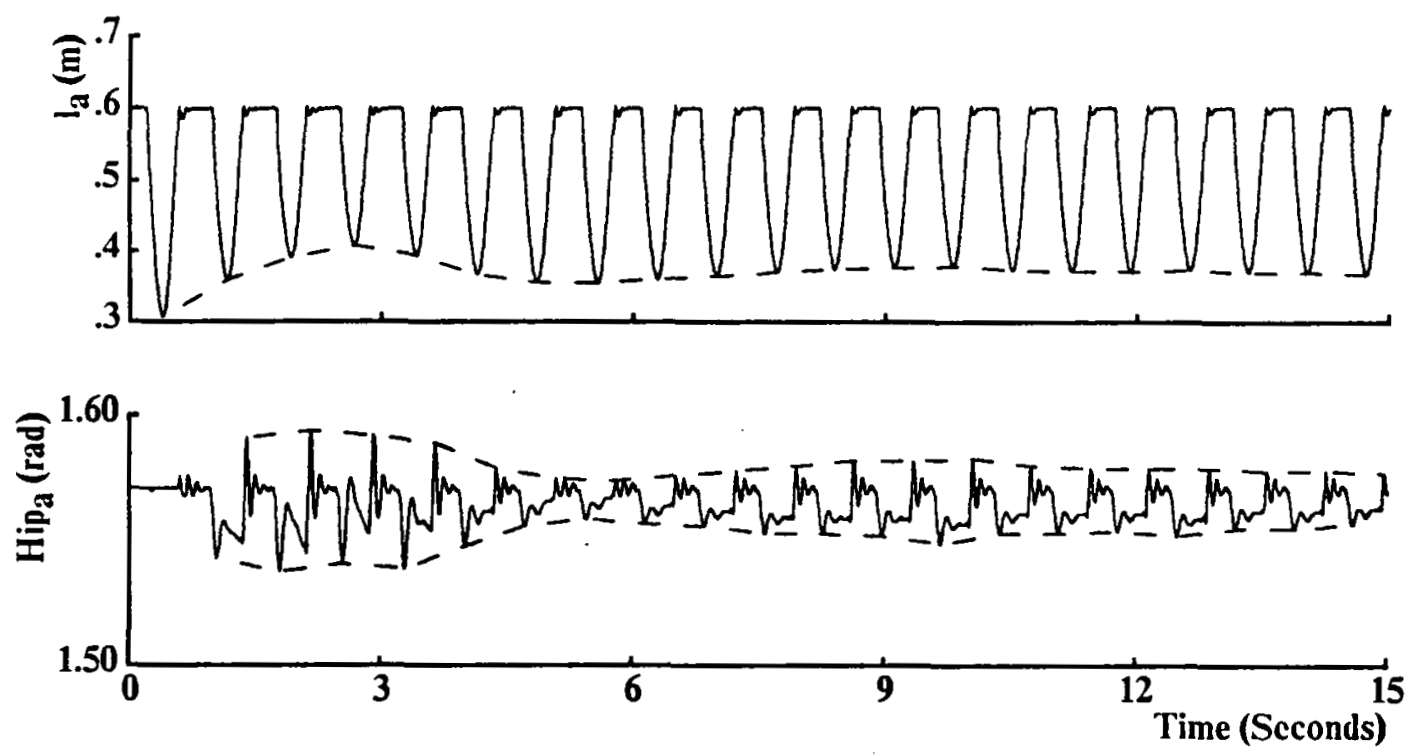

Figure 5-6: These plots show the rocking frequency that is mixed with the vertical bouncing frequency. The upper plot is leg length, the lower is the hip angle.

we varied the stiffness of the hip springs up and down to find the range of values for which periodic motion was established. We repeated the procedure for a model with stiffer legs.

For each leg stiffness there is a range of hip stiffnesses for which the model remains upright. Outside of that range it tips over. Body angle deviations of less than 0.5 radian can be damped out. Larger body angle deviations from level are not recoverable, the model always tips over.

\subsection{Discussion}

The fact that we see evidence of more than one mode of oscillation in the simulation is encouraging. There are now two things that we need to do in order to support the hypothesis that a system such as this can undergo a gait change without being explicitly forced to do so.

- We need to use different initial conditions to stimulate different modes.

- We need to tune the model to different stiffnesses and look for changes in the relationships of the modes.

It is not surprising that the vertical mode of oscillation dominates the motion of the model. This mode is excited both by the initial conditions and by the energy sources. We can excite other modes by choosing different initial hip angles. At this time we still hope to be able to differentiate gaits without having to add energy sources to the hips.

The analysis of the rocking and swaying modes relies on both feet remaining on the ground. This does not 

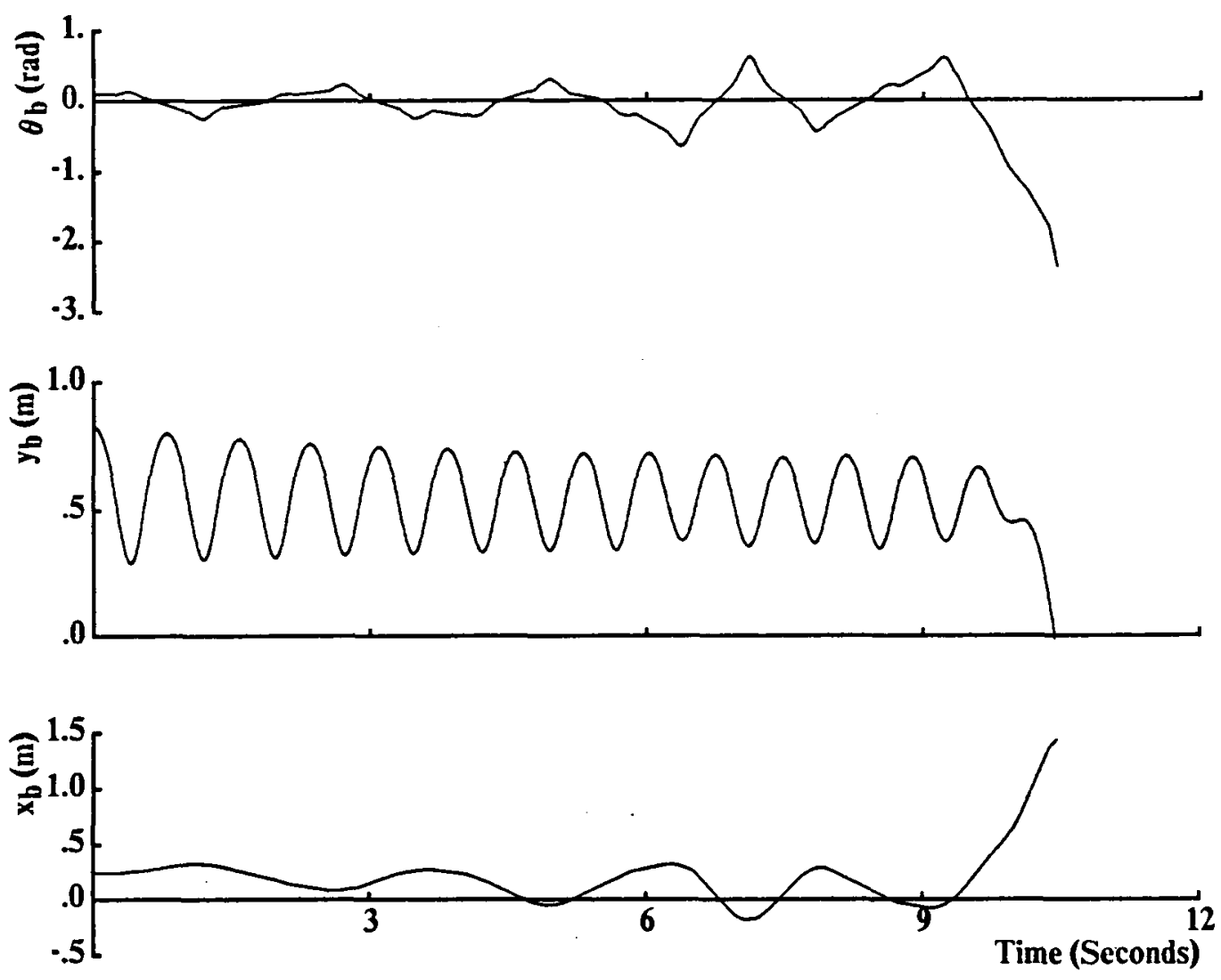

Figure 5-7: Unlike the graphs in Fig. 5-5, these graphs are from a simulation where periodic motion was not established. (Simulation r.323.5)

\begin{tabular}{llll} 
Simulation & $\mathbf{k}_{\text {hips }}$ & Frequency Ratio & Result \\
\hline r.323.1 & 1530 & 6.941 & unstable \\
r.325.2 & 1100 & 5.933 & unstable \\
r.326.1 & 910 & 5.428 & stable \\
r.320.3 & 580 & 4.417 & stable \\
r.320.9 & 320 & 3.415 & stable \\
r.323.4 & 250 & 3.090 & unstable \\
r.319.5 & 120 & 2.372 & unstable \\
& & & \\
\hline
\end{tabular}

Figure 5-2: Results of simulations with $k_{\text {legs }}=1000 \mathrm{~N} / \mathrm{m}$. The frequency ratio is the ratio of the natural frequency in rocking to the natural frequency in bouncing. The natural frequency in rocking increases as the hips are stiffened, while the natural frequency in bouncing remains constant.

happen, either in our model or in nature, because the vertical cycle requires the legs to be off of the ground part of the time. We need to analyze the effect on an oscillator of being periodically interrupted by another 


\begin{tabular}{llll} 
Simulation & $\mathrm{k}_{\text {hips }}(\mathrm{N} / \mathrm{m})$ & Frequency Ratio & Result \\
\hline r.320.8 & 1500 & 4.999 & unstable \\
r.323.3 & 1200 & 4.523 & unstable \\
r.325.1 & 880 & 3.954 & stable \\
r.313.4 & 632 & 3.449 & stable \\
r.313.3 & 417 & 2.941 & stable \\
r.312.3 & 400 & 2.897 & unstable \\
r.313.2 & 234 & 2.427 & unstable \\
\hline
\end{tabular}

Figure 5-3: Results of simulations with $k_{\text {legs }}=2000 \mathrm{~N} / \mathrm{m}$. There is a narrow range of hip stiffnesses for which the model is stable. Stable means that periodic motion is established. Unstable means that the model tips over.

oscillatory process. We believe that it is this interaction that causes the low frequency oscillation seen in the simulation data.

\subsubsection{Other Things to Study}

Animals have muscles that act at more than one joint. These two joint muscles tend to couple together the motions of adjacent joints, and may play an important part in determining the characteristic modes and frequencies of the mechanical system. Springs and dampers can be added to the model that would be roughly analogous to these muscles. This will make the model more like biological systems, and give some insight to what physical parameters are important in determining the gaits/modes of the system.

The ground doesn't have to be horizontal. Instead of adding energy, we can make the model "run down a hill". The angle of the shallowest hill that the model would run down would give a measure of "specific resistance". Specific resistance is a generalized lift-to-drag ratio used to compare the efficiency of different means of locomotion system. By changing the angle of the hill we might also cause the model to oscillate in different modes, performing a sort of "gait transition" at a certain angle.

\subsection{Summary}

Using a simple planar model, we have begun to explore the idea that gait is determined by the mechanical system that makes up an animal's body. We have analyzed some of the oscillations that occur in the model. Simulations of the model have given us a handle on stability criteria.

The simulations reveal a clear mixing of two different modes of oscillation with two different frequencies. We are now trying to tune the model so that these two modes can be made to combine in distinctly different ways, corresponding to different gaits. 


\subsection{Appendix A: Equations of Motion for the Model}

The equations of motion for the model were developed using Newtonian mechanics. There are three equations for each of the five links: summation of horizontal forces, summation of vertical forces, and summation of torques about the center of gravity. This gives fifteen equations expressing the acceleration of each link as a function of the position and velocity of the link, the spring and damper forces, the ground reaction forces, and the internal reaction forces. The internal reaction forces are eliminated leaving seven equations.

There are kinematic relationships that express the positions, velocities, and accelerations of the links in terms of the chosen state variables and their derivatives. These are substituted into the seven equations to obtain integrable expressions for the derivatives of the fourteen state variables.

The equations are expressed in matrix form:

$$
A(Q) \ddot{Q}+B(Q, \dot{Q}, R, F)=0
$$

where

Q

$\dot{\mathbf{Q}}, \ddot{\mathbf{Q}}$

$\mathbf{R}$

is the vector of the first seven state variables,

$\mathbf{F}$ are time derivatives of $Q$, is a vector of ground reactions, and is a vector of spring and damper forces.

Equation (5.23) is solved for $\ddot{\mathbf{Q}}$ :

$$
\ddot{\mathbf{Q}}=-\mathbf{A}^{-1} \mathbf{B}
$$

$\dot{\mathbf{Q}}$ and $\ddot{\mathbf{Q}}$ form the derivative of the state vector. We numerically integrate this derivative to find the new state vector. Forces, ground reactions and energies are all functions of the state.

The state variables are:

$x, \dot{x} \quad-\cdots$ The horizontal position and velocity of foot a.

$y, \dot{y} \quad-$ The vertical position and velocity of foot $a$.

$\boldsymbol{\theta}_{\mathrm{a}}, \dot{\theta}_{\mathrm{a}} \quad-$ The angle and angular velocity of leg a.

$\boldsymbol{\theta}_{b}, \dot{\theta}_{b} \quad-$ The angle and angular velocity of the body.

$\theta_{c^{\prime}} \dot{\theta}_{c} \quad-$ The angle and angular velocity of leg $c$.

$\mathrm{l}_{\mathrm{a}}, \mathfrak{l}_{\mathrm{a}} \quad-$ The length and rate of extension of leg $\mathrm{a}$.

$l_{c}, i_{c} \quad--$ The length and rate of extension of leg $c$ 


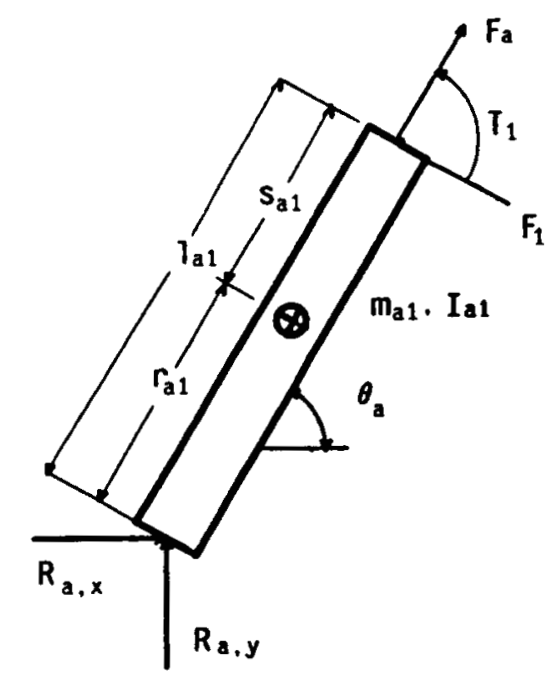

Newton's Law:

$\mathrm{T}_{1}+\mathrm{F}_{1} \mathrm{~s}_{\mathrm{al}}+\mathrm{R}_{\mathrm{a} x \mathrm{a}} \mathrm{r}_{\mathrm{al}} \sin \left(\theta_{\mathrm{a}}\right)-\mathrm{R}_{\mathrm{a}, \mathrm{y}} \mathrm{r}_{\mathrm{al}} \cos \left(\theta_{\mathrm{a}}\right)=\mathrm{I}_{\mathrm{al}} \ddot{\theta}_{\mathrm{a}}$

$\mathrm{R}_{\mathrm{a}, \mathrm{x}}+\mathrm{F}_{\mathrm{a}} \cos \left(\theta_{\mathrm{a}}\right)-\mathrm{F}_{1} \sin \left(\theta_{\mathrm{a}}\right)=\mathrm{m}_{\mathrm{al}} \ddot{\mathrm{x}}_{\mathrm{al}}$

$R_{a, y}+F_{a} \sin \left(\theta_{a}\right)+F_{1} \cos \left(\theta_{a}\right)-m_{a l} g=m_{a l} \ddot{y}_{a l}$

Kinematic relations:

$\ddot{\mathrm{y}}_{\mathrm{al}}=\ddot{\mathrm{y}}+\mathrm{r}_{\mathrm{al}}\left[\cos \left(\theta_{\mathrm{a}}\right) \ddot{\theta}_{\mathrm{a}}-\sin \left(\theta_{\mathrm{a}}\right) \dot{\theta}_{\mathrm{a}}\right]$

$\dot{y}_{\mathrm{al}}=\dot{y}+\mathrm{r}_{\mathrm{al}} \cos \left(\theta_{\mathrm{a}}\right) \dot{\theta}_{\mathrm{a}}$

$y_{\mathrm{al}}=y+r_{\mathrm{al}} \sin \left(\theta_{\mathrm{a}}\right)$

$\ddot{\mathrm{x}}_{\mathrm{al}}=\ddot{\mathrm{x}}-\mathrm{r}_{\mathrm{al}}\left[\sin \left(\theta_{\mathrm{a}}\right) \ddot{\theta}_{\mathrm{a}}+\cos \left(\theta_{\mathrm{a}}\right) \dot{\theta}_{\mathrm{a}}{ }^{2}\right]$

$\dot{x}_{\mathrm{al}}=\dot{\mathrm{x}}-\mathrm{r}_{\mathrm{al}} \sin \left(\theta_{\mathrm{a}}\right) \dot{\theta}_{\mathrm{a}}$

$\mathrm{x}_{\mathrm{al}}=\mathrm{x}+\mathrm{r}_{\mathrm{al}} \cos \left(\theta_{\mathrm{a}}\right)$

Figure 5-8: The equations of motion for the lower part of leg a

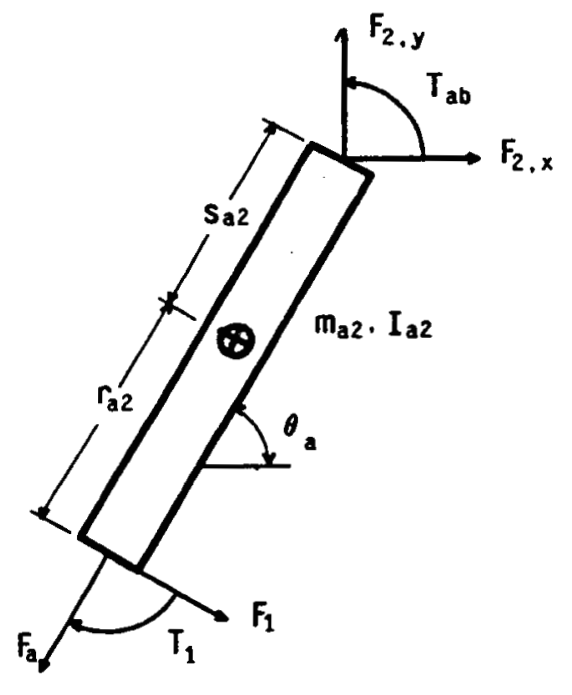

Newton's Law:

$\mathrm{F}_{1} \mathrm{r}_{\mathrm{a} 2}+\mathrm{F}_{2, \mathrm{y}} \mathrm{s}_{\mathrm{a} 2} \cos \left(\theta_{\mathrm{a}}\right)+\mathrm{T}_{\mathrm{ab}}-\mathrm{F}_{2, \mathrm{x}} \mathrm{s}_{\mathrm{a} 2} \sin \left(\theta_{\mathrm{a}}\right) \cdot \mathrm{T}_{1}=\mathrm{I}_{\mathrm{a} 2} \ddot{\theta}_{\mathrm{a}}$

$F_{2, x}+F_{1} \sin \left(\theta_{a}\right)-F_{a} \cos \left(\theta_{a}\right)=m_{a 2} \ddot{x}_{a 2}$

$F_{2, y}-F_{1} \cos \left(\theta_{a}\right)-F_{a} \sin \left(\theta_{a}\right)-m_{a 2} g=m_{a 2} \ddot{y}_{a 2}$

$\left(\mathrm{r}_{\mathrm{a} 2}=\mathrm{a}_{\mathrm{a}}-\mathrm{s}_{\mathrm{al}}-\mathrm{s}_{\mathrm{a} 2}-\mathrm{r}_{\mathrm{al}}\right)$

Kinematic relationships:

$\ddot{y}_{a 2}=\ddot{y}_{a 1}+\left(l_{a}-s_{a 2}-r_{a 1}\right)\left[\cos \left(\theta_{a}\right) \ddot{\theta}_{a}-\sin \left(\theta_{a}\right) \dot{\theta}_{a}{ }^{2}\right]+2 i_{a} \cos \left(\theta_{a}\right) \dot{\theta}_{a}+\ddot{i}_{a} \sin \left(\theta_{a}\right)$

$\dot{y}_{\mathrm{a} 2}=\dot{y}_{\mathrm{a} 1}+\left(\mathrm{l}_{\mathrm{a}}-\mathrm{s}_{\mathrm{a} 2}-\mathrm{r}_{\mathrm{al}}\right) \cos \left(\theta_{\mathrm{a}}\right) \dot{\theta}_{\mathrm{a}}+\mathrm{i}_{\mathrm{a}} \sin \left(\theta_{\mathrm{a}}\right)$

$y_{\mathrm{a} 2}=\mathrm{y}_{\mathrm{a} 1}+\left(\mathrm{a}_{\mathrm{a}}-\mathrm{s}_{\mathrm{a} 2}-\mathrm{r}_{\mathrm{al}}\right) \sin \left(\theta_{\mathrm{a}}\right)$

$\ddot{x}_{\mathrm{a} 2}=\ddot{\mathrm{x}}_{\mathrm{a} 1}-\left(\mathrm{l}_{\mathrm{a}}-\mathrm{s}_{\mathrm{a} 2}-\mathrm{r}_{\mathrm{a} 1}\right)\left[\sin \left(\theta_{\mathrm{a}}\right) \ddot{\theta}_{\mathrm{a}}+\cos \left(\theta_{\mathrm{a}}\right) \dot{\theta}_{\mathrm{a}}^{2}\right]-2 \mathrm{i}_{\mathrm{a}} \sin \left(\theta_{\mathrm{a}}\right) \dot{\theta}_{\mathrm{a}}+\ddot{i}_{\mathrm{a}} \cos \left(\theta_{\mathrm{a}}\right)$

$\dot{x}_{\mathrm{a} 2}=\dot{x}_{\mathrm{a} 1}-\left(\mathrm{l}_{\mathrm{a}}-\mathrm{s}_{\mathrm{a} 2}-\mathrm{r}_{\mathrm{al}}\right) \sin \left(\theta_{\mathrm{a}}\right) \dot{\theta}_{\mathrm{a}}+\mathrm{i}_{\mathrm{a}} \cos \left(\theta_{\mathrm{a}}\right)$

$x_{a 2}=x_{a 1}+\left(l_{a}-s_{a 2}-r_{a l}\right) \cos \left(\theta_{a}\right)$

Figure 5-9: The equations of motion for the upper part of leg a 


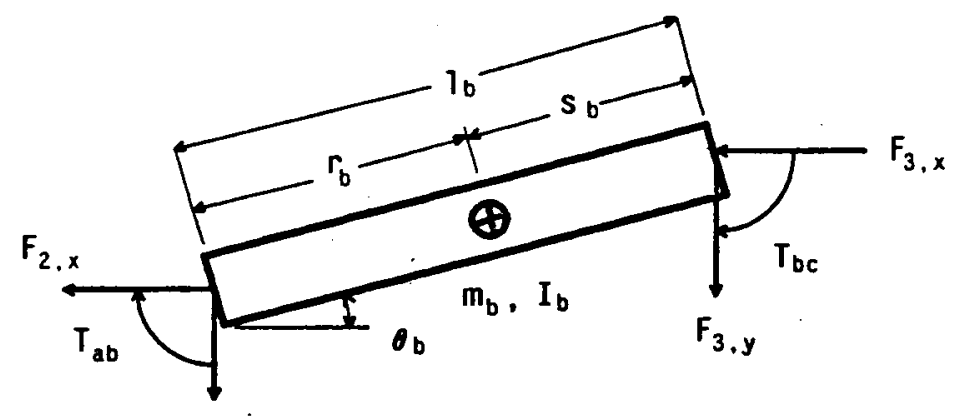

$F_{2, y}$

Newton's Law:

$-T_{b c}-T_{a b}+F_{2, y} r_{b} \cos \left(\theta_{b}\right)-F_{3, y} s_{b} \cos \left(\theta_{b}\right)-F_{2, x} r_{b} \sin \left(\theta_{b}\right)+F_{3, x} s_{b} \sin \left(\theta_{b}\right)=I_{b} \ddot{\theta}_{b}$

$-F_{3, x}-F_{2, x}=m_{b} \ddot{x b}$

$-F_{3, y} \cdot F_{2, y}-m_{b} g=m_{b} \ddot{y}_{b}$

Kinematic relationships:

$\ddot{y}_{b}=\ddot{y}_{a 2}+s_{a 2}\left[\cos \left(\theta_{a}\right) \ddot{\theta}_{a}-\sin \left(\theta_{a}\right) \dot{\theta}_{a}{ }^{2}\right]+r_{b}\left[\cos \left(\theta_{b}\right) \ddot{\theta}_{b}-\sin \left(\theta_{b}\right) \dot{\theta}_{b}{ }^{2}\right]$

$\dot{y}_{b}=\dot{y}_{a 2}+s_{a 2} \cos \left(\theta_{a}\right) \dot{\theta}_{a}+r_{b} \cos \left(\theta_{b}\right) \dot{\theta}_{b}$

$y_{b}=y_{a 2}+s_{a 2} \sin \left(\theta_{a}\right)+r_{b} \sin \left(\theta_{b}\right)$

$\ddot{x}_{b}=\ddot{x}_{a 2}-s_{a 2}\left[\sin \left(\theta_{a}\right) \ddot{\theta}_{a}+\cos \left(\theta_{a}\right) \dot{\theta}_{a}{ }^{2}\right]-r_{b}\left[\sin \left(\theta_{b}\right) \ddot{\theta}_{b}+\cos \left(\theta_{b}\right) \dot{\theta}_{b}{ }^{2}\right]$

$\dot{x}_{b}=\dot{x}_{a 2}-s_{a 2} \sin \left(\theta_{a}\right) \dot{\theta}_{a}-r_{b} \sin \left(\theta_{b}\right) \dot{\theta}_{b}$

$\mathrm{x}_{\mathrm{b}}=\mathrm{x}_{\mathrm{a} 2}+\mathrm{s}_{\mathrm{a} 2} \cos \left(\theta_{\mathrm{a}}\right)+\mathrm{r}_{\mathrm{b}} \cos \left(\theta_{\mathrm{b}}\right)$

Figure 5-10: The equations of motion for the body. 


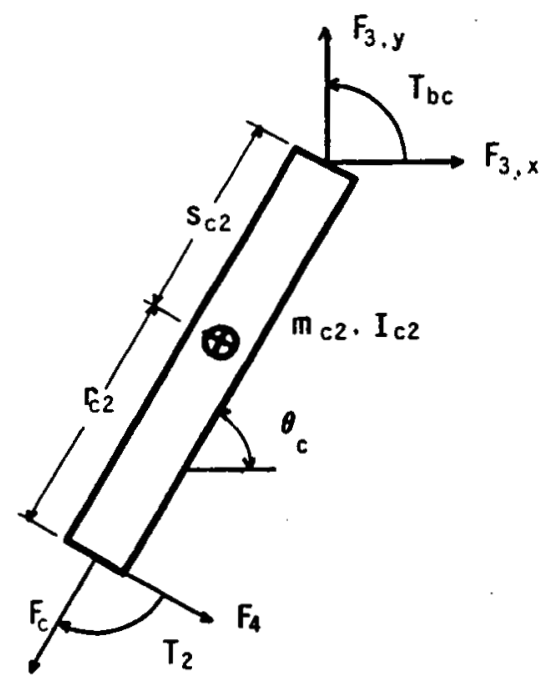

Newton's Law:

$\mathrm{F}_{4} \mathrm{r}_{\mathrm{c} 2}+\mathrm{F}_{3, \mathrm{y}} \mathrm{s} 2 \cos \left(\theta_{c}\right)-\mathrm{F}_{3, \mathrm{x}} \mathrm{s}_{\mathrm{c} 2} \sin \left(\theta_{\mathrm{c}}\right)+\mathrm{T}_{\mathrm{bc}}-\mathrm{T}_{2}=\mathrm{I}_{\mathrm{c} 2} \ddot{\theta}_{\mathrm{c}}$

$F_{3, x}+F_{4} \sin \left(\theta_{c}\right)-F_{c} \cos \left(\theta_{c}\right)=m_{c 2} \ddot{x}_{c 2}$

$F_{3, y}-F_{4} \cos \left(\theta_{c}\right)-F_{c} \sin \left(\theta_{c}\right)-m_{c 2} g=m_{c 2} \dot{y}_{c 2}$

Kinematic relationships:

$\ddot{y}_{c 2}=\ddot{y}_{b}+s_{b}\left[\cos \left(\theta_{b}\right) \ddot{\theta}_{b}-\sin \left(\theta_{b}\right) \dot{\theta}_{b}{ }^{2}\right]-s_{c 2}\left[\cos \left(\theta_{c}\right) \ddot{\theta}_{c}-\sin \left(\theta_{c}\right) \dot{\theta}_{c}{ }_{c}\right]$

$\dot{y}_{c 2}=\dot{y}_{b}+s_{b} \cos \left(\theta_{b}\right) \dot{\theta}_{b}-s_{c 2} \cos \left(\theta_{c}\right) \dot{\theta}_{c}$

$y_{c 2}=y_{b}+s_{b} \sin \left(\theta_{b}\right)-s_{c 2} \sin \left(\theta_{c}\right)$

$\ddot{x}_{c 2}=\ddot{x}_{b}-s_{b}\left[\sin \left(\theta_{b}\right) \ddot{\theta}_{b}+\cos \left(\theta_{b}\right) \dot{\theta}_{b}{ }^{2}\right]+s_{c 2}\left[\sin \left(\theta_{c} \ddot{\theta}_{c}+\cos \left(\theta_{c}\right) \dot{\theta}_{c}{ }^{2}\right]\right.$

$\dot{x}_{\mathrm{c} 2}=\dot{x}_{\mathrm{b}}-s_{\mathrm{b}} \sin \left(\theta_{\mathrm{b}}\right) \dot{\theta}_{\mathrm{b}}+\mathrm{s}_{\mathrm{c} 2} \sin \left(\theta_{\mathrm{c}}\right) \dot{\theta}_{\mathrm{c}}$

$x_{c 2}=x_{b}+s_{b} \cos \left(\theta_{b}\right)-s_{c 2} \cos \left(\theta_{c}\right)$

Figure 5-11: The Equations of motion for the upper part of leg $c$

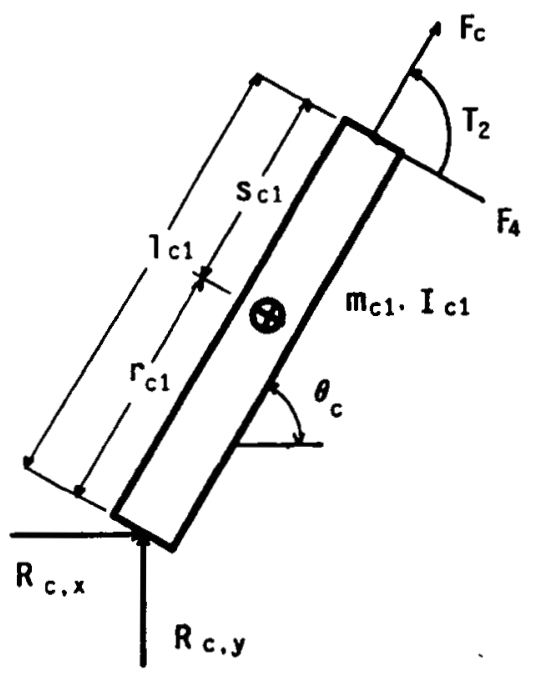

Newton's Law:

$\mathrm{T}_{2}+\mathrm{F}_{4} \mathrm{~s}_{\mathrm{cl}}+\mathrm{R}_{\mathrm{c}, \mathrm{x}} \mathrm{r}_{\mathrm{cl}} \sin \left(\theta_{\mathrm{c}}\right)-\mathrm{R}_{\mathrm{c}, \mathrm{y}} \mathrm{r}_{\mathrm{cl}} \cos \left(\theta_{\mathrm{c}}\right)=\mathrm{I}_{\mathrm{cl}} \ddot{\theta}_{\mathrm{c}}$

$R_{c, x}+F_{c} \cos \left(\theta_{c}\right)-F_{4} \sin \left(\theta_{c}\right)=m_{c l} \ddot{x}_{c l}$

$R_{c, y}+F_{c} \sin \left(\theta_{c}\right)+F_{4} \cos \left(\theta_{c}\right)-m_{c l} g=m_{c l} \ddot{y}_{c l}$

$\left(\mathrm{r}_{\mathrm{c} 2}=1_{\mathrm{c}} \cdot \mathrm{s}_{\mathrm{cl}} \cdot \mathrm{s}_{\mathrm{c} 2}-\mathrm{r}_{\mathrm{cl}}\right)$

Kinematic relationships:

$\ddot{y}_{c 1}=\ddot{y}_{c 2}-\left(1_{c}-s_{c 2}-r_{c 1}\right)\left[\cos \left(\theta_{c} \ddot{\theta}_{c}-\sin \left(\theta_{c} \dot{\theta}_{c}{ }_{c}\right]-2 \dot{i}_{c} \cos \left(\theta_{c}\right) \dot{\theta}_{c}-\ddot{i}_{c} \sin \left(\theta_{c}\right)\right.\right.$

$\dot{y}_{c 1}=\dot{y}_{c 2}-\left(l_{c}-s_{c 2}-r_{c 1}\right) \cos \left(\theta_{c}\right) \dot{\theta}_{c}-i_{c} \sin \left(\theta_{c}\right)$

$y_{c 1}=y_{c 2}-\left(l_{c}-s_{c 2}-r_{c 1}\right) \sin \left(\theta_{c}\right)$

$\ddot{x}_{c 1}=\ddot{y}_{c 2}+\left(a_{c}-s_{c 2}-t_{c 1}\right)\left[\sin \left(\theta_{c}\right) \dot{\theta}_{c}+\cos \left(\theta_{c} \dot{\theta}_{c}{ }^{2}\right]+2 i_{c} \sin \left(\theta_{c}\right) \dot{\theta}_{c}-\ddot{i}_{c} \cos \left(\theta_{c}\right)\right.$

$\dot{x}_{c 2}=\dot{x}_{c 2}+\left(1_{c}-s_{c 2}-r_{c 1}\right) \sin \left(\theta_{c}\right) \dot{\theta}_{c}-i_{c} \cos \left(\theta_{c}\right)$

$\mathrm{x}_{\mathrm{cl}}=\mathrm{x}_{\mathrm{c} 2}-\left(\mathrm{l}_{\mathrm{c}}-\mathrm{s}_{\mathrm{c} 2}-\mathrm{r}_{\mathrm{cl}}\right) \cos \left(\theta_{\mathrm{c}}\right)$

Figure 5-12: The equations of motion for the lower part of leg c 


\subsection{Appendix B: Simulation Parameters}

Parameter Value

Description

Parameters for the lower part of the legs

$\mathrm{s}_{\mathrm{al}}, \mathrm{s}_{\mathrm{cl}}$

$\mathrm{r}_{\mathrm{al}}, \mathrm{r}_{\mathrm{cl}}$

$\mathrm{I}_{\mathrm{al}}, \mathrm{I}_{\mathrm{cl}}$

$\mathrm{m}_{\mathrm{al}}, \mathrm{m}_{\mathrm{cl}}$
0.10 meters

0.10 meters

$0.0004 \mathrm{~kg}-\mathrm{m}^{2}$

$0.50 \mathrm{~kg}$
Distance from the center of gravity to the upper end.

Distance from the foot to the center of gravity.

Moment of Inertia.

Mass.

Parameters for the upper part of the legs:

$\begin{array}{lll}\mathrm{s}_{\mathrm{a} 2}, \mathrm{~s}_{\mathrm{c} 2} & 0.10 \text { meters } & \text { Distance from the center of gravity to the hip. } \\ \mathrm{I}_{\mathrm{a} 2}, \mathrm{I}_{\mathrm{c} 2} & 0.0004 \mathrm{~kg}-\mathrm{m}^{2} & \text { Moment of Inertia. } \\ \mathrm{m}_{\mathrm{a} 2}, \mathrm{~m}_{\mathrm{c} 2} & 0.50 \mathrm{~kg} & \text { Mass. }\end{array}$

Parameters for the body:

$\begin{array}{lll}\mathrm{s}_{\mathrm{b}}, \mathrm{r}_{\mathrm{b}} & 0.30 \text { meters } & \text { Distance from each hip to the center of gravity. } \\ \mathrm{I}_{\mathrm{b}} & 0.54 \mathrm{~kg}-\mathrm{m}^{2} & \text { Moment of Inertia. } \\ \mathrm{m}_{\mathrm{b}} & 18.00 \mathrm{~kg} & \text { Mass. }\end{array}$

Parameters of the ground:

$\begin{array}{lll}\mathrm{k}_{\mathrm{g}, \mathrm{x}}, \mathrm{k}_{\mathrm{g}, \mathrm{y}} & 50000.00 \mathrm{~N} / \mathrm{m} & \begin{array}{l}\text { Stiffness of the ground. } \\ \mathrm{b}_{\mathrm{g}, \mathrm{x}}, \mathrm{b}_{\mathrm{g}, \mathrm{y}}\end{array} \\ \mathrm{g} & 100.00 \mathrm{~N}-\mathrm{s} / \mathrm{m} & \text { Viscosity of the ground. } \\ \mathrm{g}_{\mathrm{c}} & 9.81 \mathrm{~m} / \mathrm{sec}^{2} & \text { The acceleration of gravity. }\end{array}$

Parameters of the springs and dampers:

$k_{a}, k_{c}$

$k_{a b}, k_{b c}$

$b_{a}, b_{c}$

$\mathrm{b}_{\mathrm{ab}}, \mathrm{b}_{\mathrm{bc}}$

$1_{2,}, 1_{c, 0}$

$1_{\text {a, }, 0, s r c^{\prime}}{ }^{c} c, 0$, src

$\theta_{\mathrm{ab}, 0}, \theta_{\mathrm{bc}, 0}$

$1000.00 \mathrm{~N} / \mathrm{m}$

$400.00 \mathrm{~N} \cdot \mathrm{m} / \mathrm{rad}$

$5.00 \mathrm{~N}-\mathrm{s} / \mathrm{m}$

$5.00 \mathrm{~N}-\mathrm{m}-\mathrm{s} / \mathrm{rad}$

0.60 meters

0.62 meters

1.57 radians

Parameters of the stops:

$l_{a_{\text {max }}}, 1_{a, \min }$
$1_{c, \max }, 1_{c, \min }$
$k_{\text {stops }}$
$b_{\text {stops }}$

0.20 meters

0.20 meters

$50000.00 \mathrm{~N} / \mathrm{m}$

$100.00 \mathrm{~N}-\mathrm{s} / \mathrm{m}$
The stiffness of the legs.

The stiffness of the hips.

The viscosity of the legs.

The viscosity of the hips.

Rest length of the legs.

Modified rest length for the legs.

Rest angle of the hips.

Maximum length of the legs.

Minimum length of the legs.

Stiffness of the stops.

Viscosity of the stops. 


\section{Path Control in 3D}

\section{Seshashayee S. Murthy}

\subsection{Int roduction}

The ability to traverse an arbitrary path in the horizontal plane is an important milestone in achieving maneuverability and avoiding obstacles in the path of the hopper. To follow an arbitrary curve in the horizontal plane it is necessary to alter the direction of motion of the hopper. We describe here a method that uses lateral foot placement to alter the direction of motion of the hopper when it is in motion. The ability to alter the direction of motion while the hopper is not stationary is important for maneuverability and speed. In the absence of such a scheme, changing the direction of travel requires that the hopper be first brought to a stop before motion is initiated in a new direction. Fig. 6-1 illustrates this form of path control. The desired path is a square with $2 \mathrm{~m}$ sides. The hopper takes $24 \mathrm{sec}$ for the circuit but the accuracy of the path is quite good.

Altering the direction of travel of the hopper while it is in motion requires the application of a force perpendicular to the plane of motion. There are two ways to bring such a force to bear on the hopper.

1. Placement of the foot outside the plane of motion causes a lateral force to act on the hopper. If the foot is not under the center of gravity, the body accelerates away from the foot because of forces acting in that direction. Fig. 6-2 describes how these forces act.

- At touch-down, an impact force acts on the foot. The magnitude and direction of this force are determined by the forward velocity at touch-down and the distance of the foot from the plane of motion.

- Also, during stance, because of such asymmetrical placement, gravity causes a torque along the roll axis, at the foot. This causes the hopper to accelerate in a direction perpendicular to the line of motion.

- Thirdly, during the stance period an upward force is being applied on the body by the leg. Because the leg is at an angle with respect to the plane of motion the resultant ground reaction forces have a component perpendicular to the plane of motion. Placement of the foot outside the plane of motion thus causes lateral forces on the hopper that result in a lateral acceleration.

\section{Hip torques during stance.}

During the stance phase the foot is constrained not to move due to friction. Therefore, torques applied at the hip cause reaction forces at the ground. Hip torques along the roll axis cause reaction forces perpendicular to the line of motion. These reaction forces can be used to alter the velocity of the hopper. 


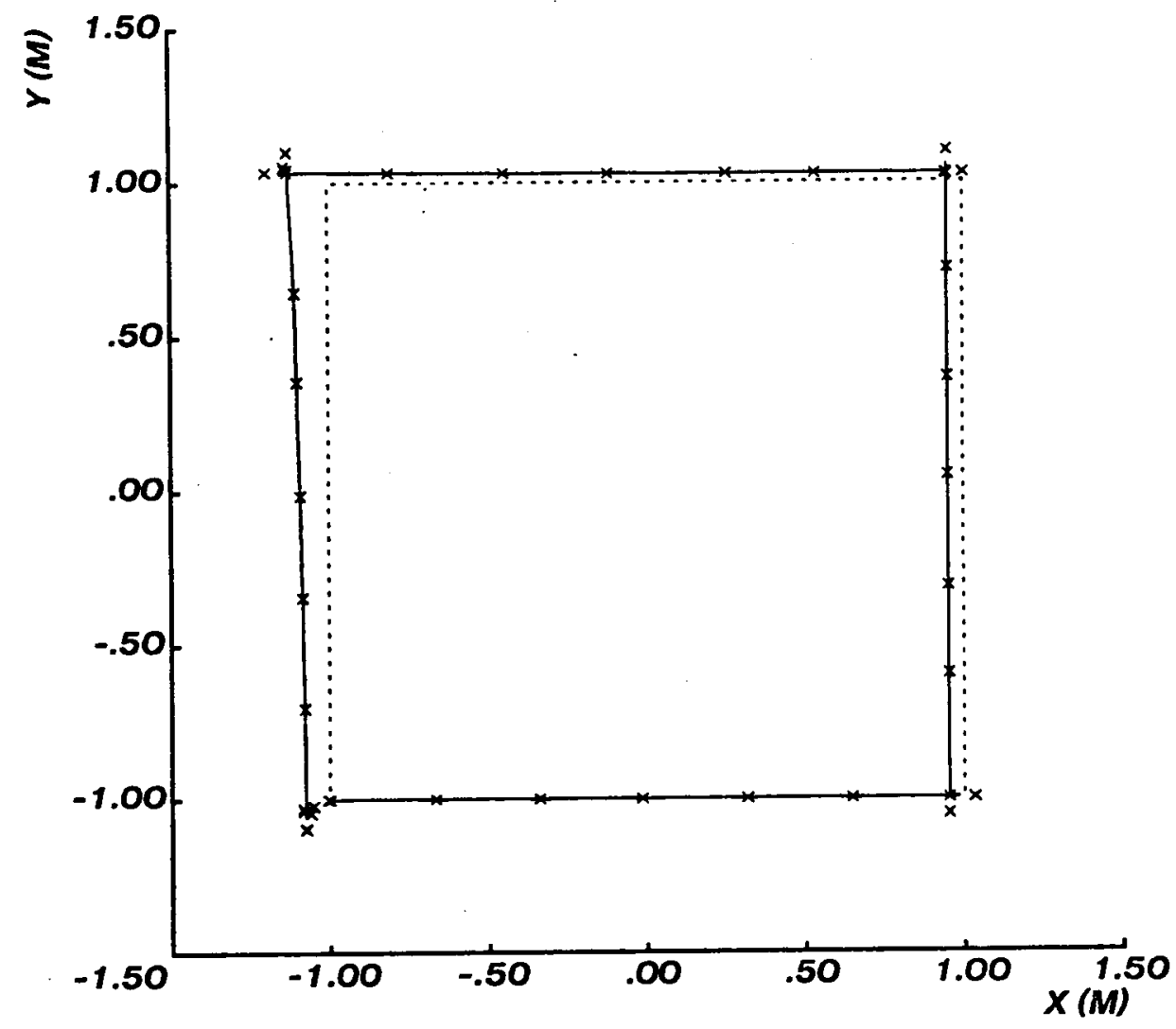

Figure 6-1: Path control. The 3D model was made to follow a square path. It started at $(-1,-1)$, lower left, and progressed anti-clockwise through $(1,-1),(1,1)$, and $(-1,1)$, finally returning to the starting point. Total time around the square was $24 \mathrm{sec}$.

\subsection{Path Control Using Foot Placement}

The change in the momentum of the hopper, $\Delta \mathbf{M}$, during the stance phase can be expressed as a function of the placement of the foot in the horizontal plane, the forward and vertical velocities at touch-down and the torques applied during the stance phase. 

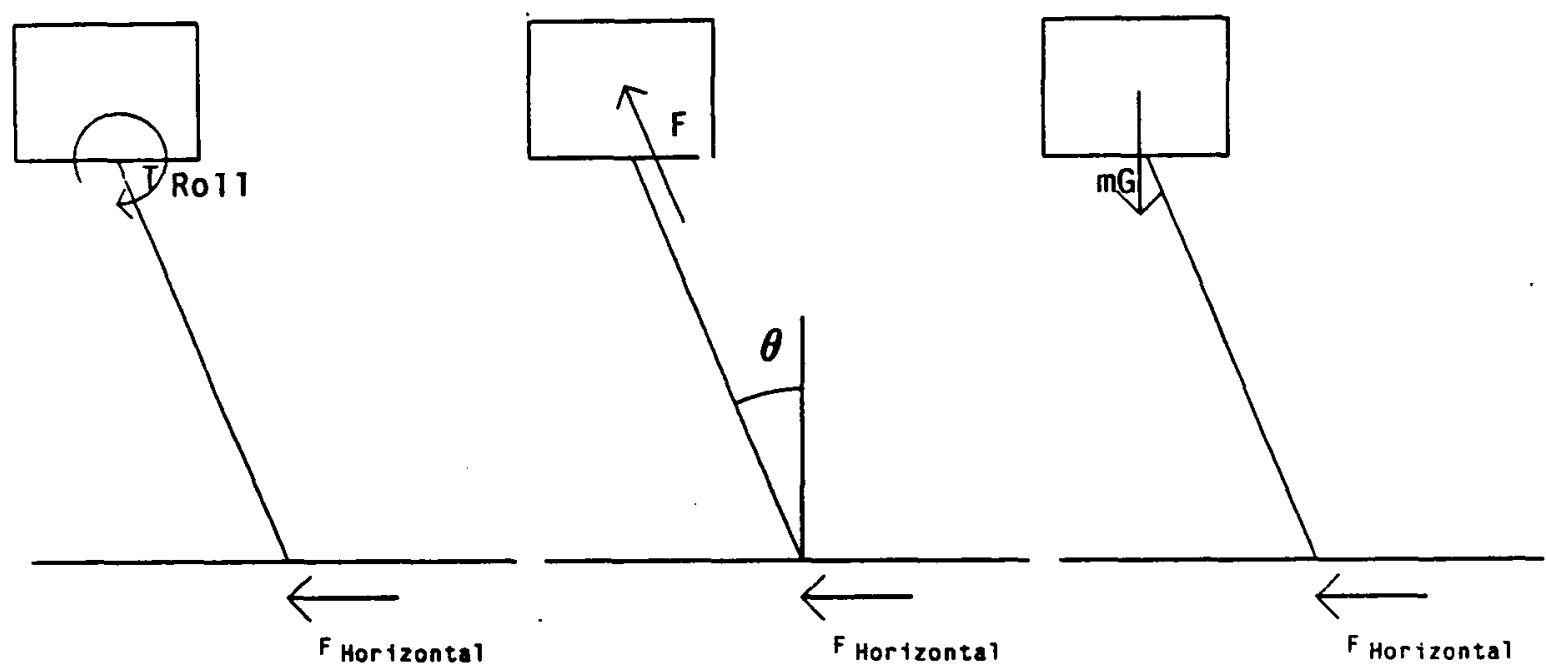

Figure 6-2: Lateral forces acting on the foot. Left) The effect of hip torques on the hopper. A roll torque at the hip causes a reaction force in the lateral direction at the foot. Center) The effect of force applied at the linear actuator at the hip. Because the force $F$ acts at an angle $\theta$, it has a component $F$ $\sin \theta$ in the lateral dircetion. This causes an equal reaction force at the ground. Right) The effect of gravity on the hopper. Because the sum of moments at the foot has to be zero, there is a ground reaction force acting the foot.

$\Delta M=f\left(D_{\text {Lat }}, D_{\text {Long }}, T_{\text {Hip }}, V_{\text {Long }}, V_{\text {Ver }}\right)$
where

$\mathrm{D}_{\text {Lat }}$ is the lateral distance of the foot from the plane of motion at touch-down.

$\mathrm{D}_{\text {Long }}$ is the longitudinal distance of the foot from the center of gravity along the direction of motion at touch-down.

$\mathbf{V}_{\text {Long }}$ is the velocity of the hopper in the horizontal plane at touch-down.

$V_{\text {Ver }}$ is the vertical velocity of the hopper.

$\mathrm{T}_{\text {Hip }}$ are the torques applied on the body at the hip.

To achieve path control in the plane, we need to find, prior to touch-down, the correct control actions that would result in the desired $\Delta \mathbf{M}$. These could be placement of the foot or application of hip torques during stance. We have not found an analytical method to predict $\Delta \mathbf{M}$. One alternative would be to simulate the hopper, an open loop linkage with 6 joints, for the duration of the stance phase. To achieve effective real-time control it is necessary to complete these computations in about $50 \mathrm{~ms}$ so that the rest of the flight phase can be used to orient the leg correctly. This is a tremendous computational burden. It would be preferable to find a less computation intensive control scheme.

The approach I am presently investigating is an extension of the controller used for straight line running. It 

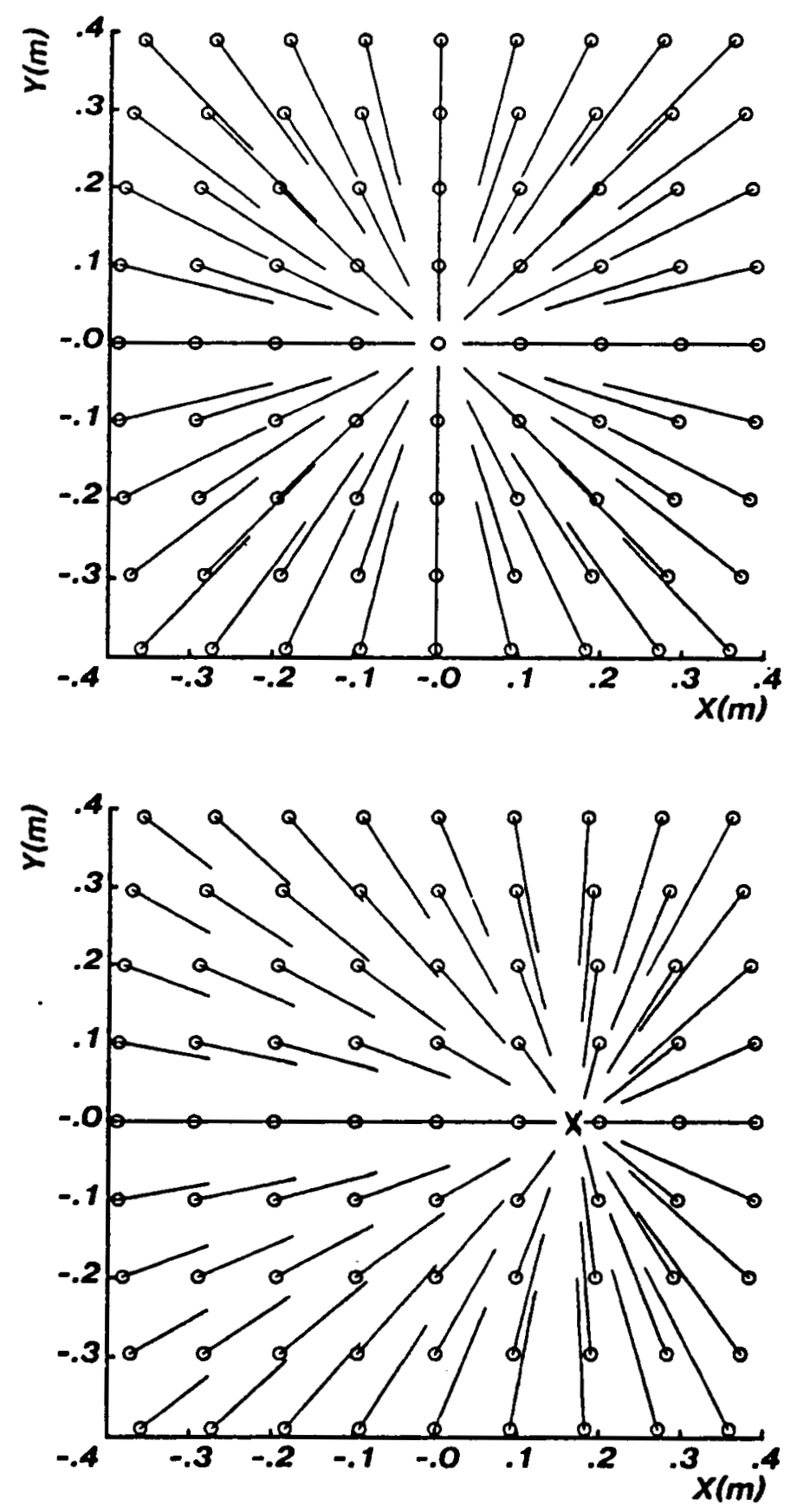

Figure 6-3: Effect of foot placement on the velocity of the hopper. The circles mark the position of the foot with respect to the center of gravity of the hopper at touch-down. The vectors represent the change in the velocity of the hopper for each foot placement during the stance phase. In the top figure the hopper has no forward velocity at touch-down. The acceleration vectors all point towards the origin. The length of the vectors increases with the distance from the origin. In the bottom figure the forward velocity at touch-down is $1 \mathrm{~m} / \mathrm{sec}$ in the positive $x$ direction. The $x$ marks the foot placement that results in zero velocity change. The acceleration vectors point in this general direction. For small displacements the magnitude of the acceleration vectors increases with the distance from this point.

uses foot placement at touch-down alone to achieve the desired velocity changes. Using a series of 


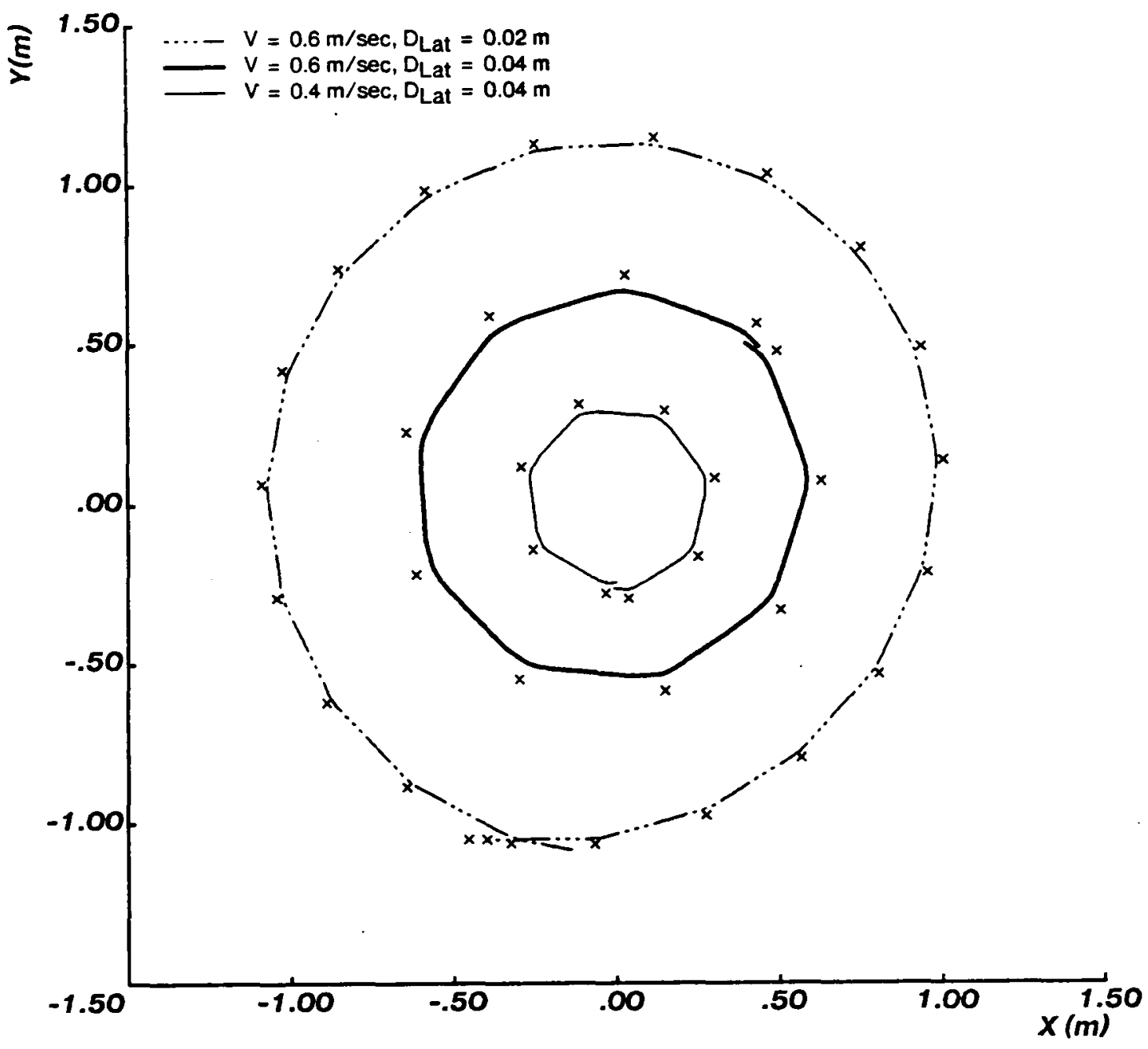

Figure 6-4: Circular trajectories. The hopper follows a circular trajectory if $\mathrm{D}_{\text {Lat }}$ and the forward speed are both maintained constant during each hop. The curves show the path followed by the center of gravity of the hopper while it is traversing three such circles. The $x$ 's mark the points at which the foot touched down during stance. The middle circle is traversed when the speed is $0.6 \mathrm{~m} / \mathrm{sec}$ and $\mathrm{D}_{\mathrm{Lat}}$ is maintained at $0.04 \mathrm{~m}$. If $\mathrm{D}_{\mathrm{Lat}}$ is decreased to $0.02 \mathrm{~m}$ while the specd remains fixed at $0.6 \mathrm{~m} / \mathrm{sec}$ the lateral acceleration decreases. The hopper therefore traverses the outer circle which has a larger radius. If $\mathrm{D}_{\text {Lat }}$ is maintained at $0.04 \mathrm{~m}$ while the speed is decreased to $0.4 \mathrm{~m} / \mathrm{sec}$ the change in direction during each stance phase is more pronounced. The hopper therefore traverses a circle which has a smaller radius.

simulations it is possible to characterize the acceleration of the hopper during the stance phase as a function of the foot placement, given that hip torques are at the values dictated by the controller. It is therefore possible to find a tabular representation of Equation (6.1) where the hip torques are factored out by assuming that they are at their nominal values during stance.

Fig. 6-3 plots the acceleration vector of the center of gravity of the hopper as a function of foot placement for 


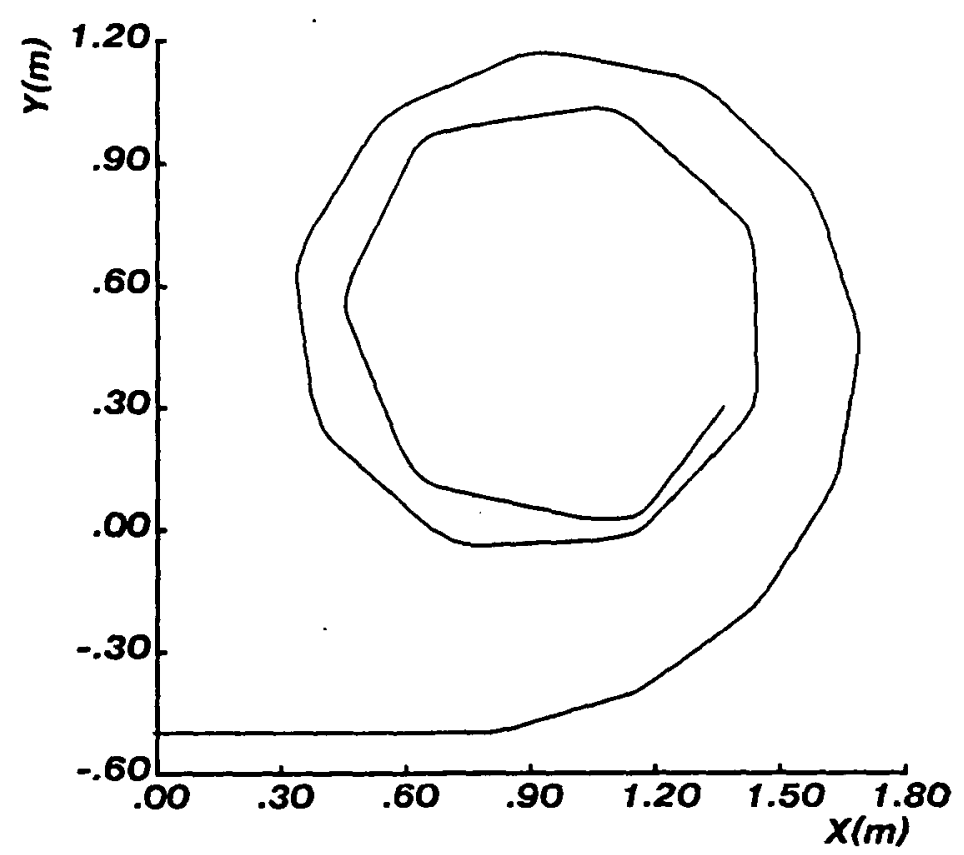

Figure 6-5: Spiral Trajectories. The lateral acceleration during the stance phase increases with the lateral displacement of the foot. If the displacement is increased each successive hop, then the hopper follows a spiral trajectory. Here the forward speed was fixed at $0.6 \mathrm{~m} / \mathrm{sec}$. $D_{\text {Lat }}$ was increased from 0.0 to $0.035 \mathrm{~m}$

two different forward velocities. Hip torques are at their nominal values. The circles mark the position at touch-down of the foot with respect to the center of gravity The forward velocity at touch-down is along the $x$ axis. The hopper resembles an inverted pendulum that is falling towards the ground. In Fig. 6-3 (top) the hopper has no forward velocity. After the foot touches the ground the hopper falls away from the point of touch-down towards the center of gravity. The graph is therefore radially symmetric about the origin. The forward velocity imparted during the stance phase increases with the distance from the origin. In Fig. 6-3 (bottom) the forward velocity at touch-down is $1.0 \mathrm{~m} / \mathrm{sec}$ in the $x$ direction. Here the $x$ marks the point of zero acceleration. The acceleration vectors point in this general direction. The lateral acceleration and the longitudinal acceleration are almost independent of each other when the lateral displacement is small compared to the longitudinal displacement of the foot.

Using a systematic series of such simulations, I have built a table to predict the acceleration of the hopper as a function of the foot placement and the velocity at touch-down. The forward and the vertical velocities at touch-down can be calculated at the previous lift-off. The desired longitudinal and lateral accelerations, are calculated from the desired trajectory. These four quantities can then be used as indices into a table, to find the foot placement that results in the desired accelerations during stance. By using this method it is therefore possible to cause the hopper to follow a desired path. 


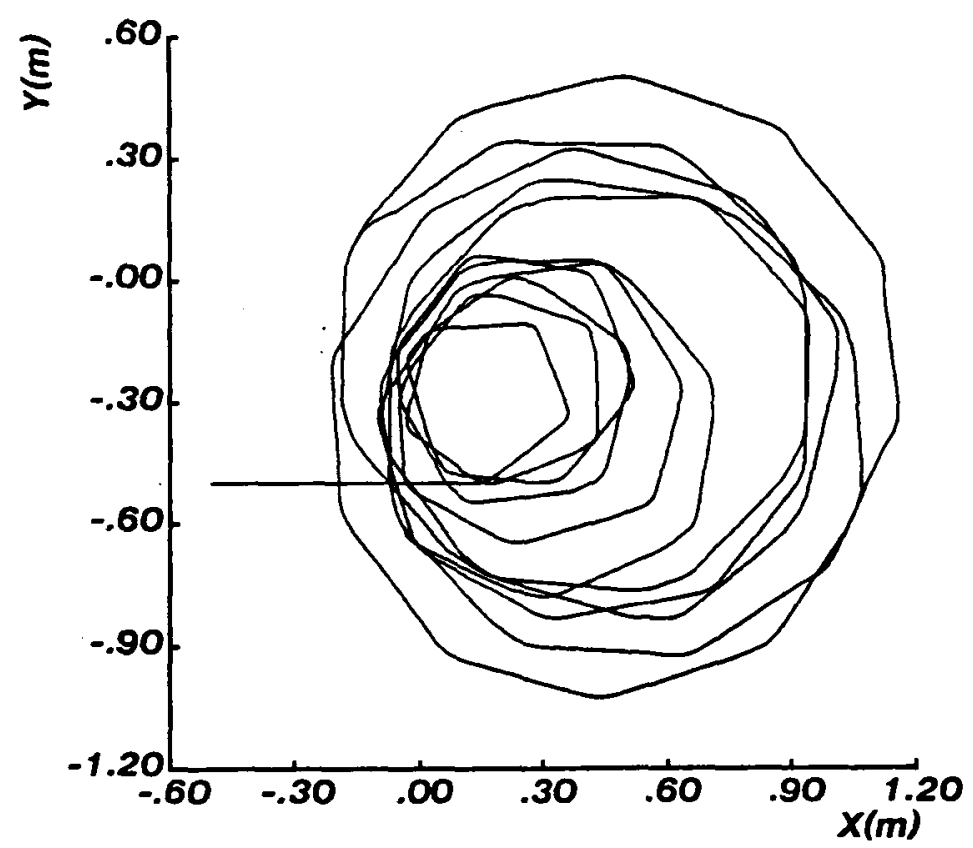

Figure 6-6: Spiral Trajectories. Keeping $D_{\text {Lat }}$ constant while continuously increasing the forward velocity results in a continuous increase in the radius of curvature. The hopper therefore traverses a spiral in the outward direction. Here, the forward velocity was increased from $0.2 \mathrm{~m} / \mathrm{sec}$ to $1.0 \mathrm{~m} / \mathrm{sec}$ while $D_{\text {Lat }}$ remains fixed at $0.04 \mathrm{~m}$.

\subsection{Trajectories}

We have used asymmetrical placement of the foot to cause the hopper to follow various trajectories. These trajectories show that hopper can be made to follow an arbitrary path with a varying radius of curvature.

\subsubsection{Circles}

A constant lateral acceleration is necessary to cause the hopper to follow a circular trajectory. From the discussion in the previous section and Fig. 6-3 we can infer that if the speed of the hopper and the distance $\mathrm{D}_{\mathrm{Lat}}$ are maintained constant, then the change in velocity during each stance phase is the same. The hopper experiences a constant centripetal acceleration during the stance phase. During the flight phase the trajectory of the center of gravity is a straight line because no external forces act on the hopper. The hopper can thus be caused to follow a path that closely resembles a circle. Fig. 6-4 is a plot of the path of the center of gravity of the hopper. The horizontal speed of the hopper was maintained constant at $0.6 \mathrm{~m} / \mathrm{sec}$ and the distance $\mathrm{D}_{\text {Lat }}$ was maintained constant at $0.04 \mathrm{~m}$. The resultant trajectory closely approximates a circle.

We can infer from Fig. 6-3 that if $D_{\text {Lat }}$ is decreased while the hopper's speed is maintained constant, then the lateral acceleration decreases. The change in direction is therefore less pronounced and the hopper traverses a circle of a larger radius. In Fig. 6-4 the hopper traverses the outer circle at the same speed , $0.6 \mathrm{~m} / \mathrm{sec}$ as the middle circle but $D_{\mathrm{Lat}}$ is maintaincd constant at $0.02 \mathrm{~m}$. 


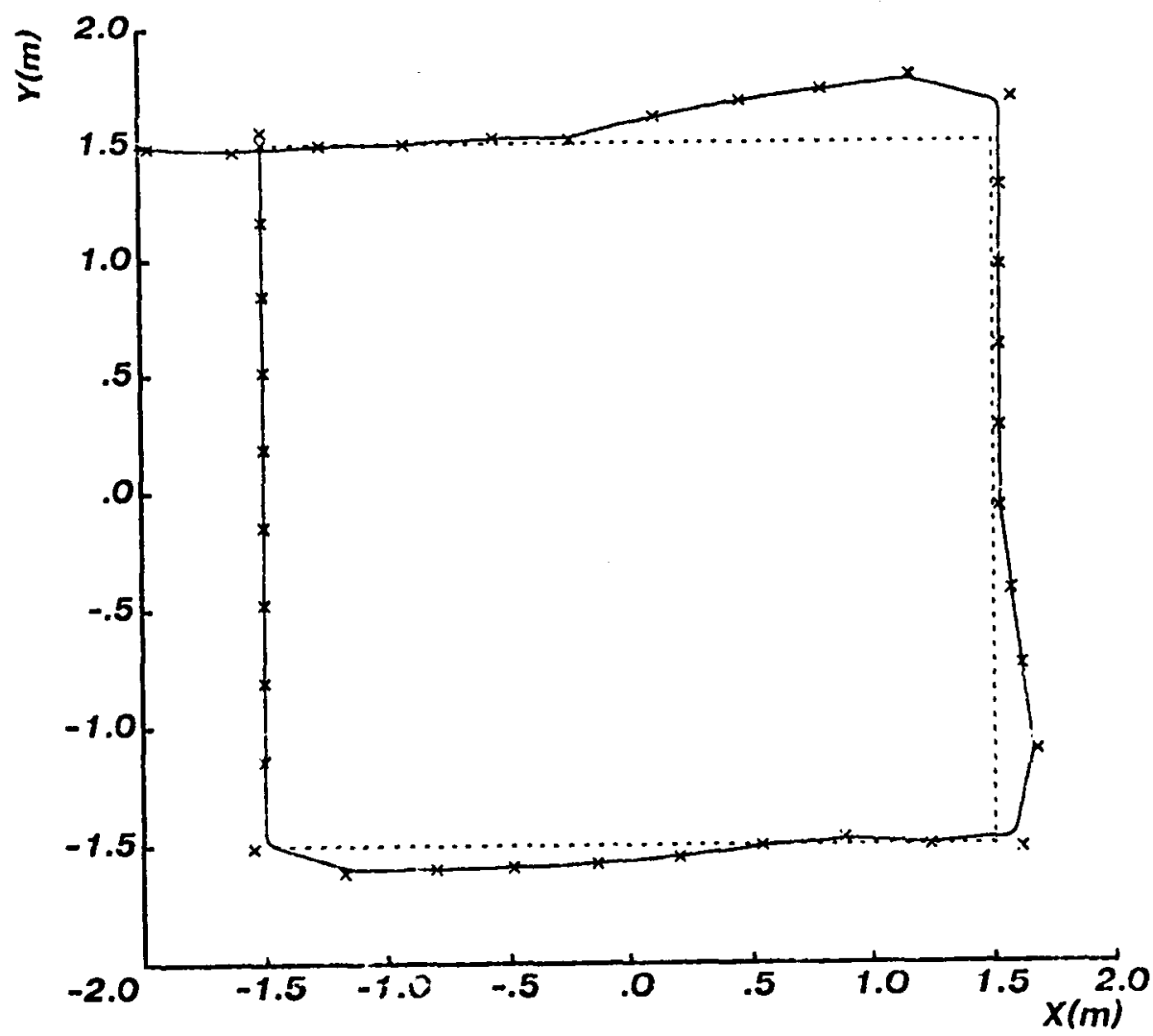

Figure 6-7: A square path: The continuous dark line represents the path followed by the hopper while attempting to traverse a square path with sides $3 \mathrm{~m}$ in length. The dotted line represents the desired trajectory. The x's mark the points at which the foot was placed during stance. The hopper starts off at the top left-hand side corner and traverses the path in an anti-clockwise fashion at a constant forward velocity of $0.7 \mathrm{~m} / \mathrm{sec}$. At each of the corners the hopper has to make a sharp turn. To achieve this the foot is placed ahead and to the right of the point of zero acceleration. The forward velocity changes direction by almost a right angle as a result. The quick turns reduce the time around the circuit to $19 \mathrm{sec}$.

If $\mathrm{D}_{\text {Lat }}$ is maintained constant while the speed of the hopper is decreased then the change in direction is more pronounced and the hopper traverses a circle with a smaller radius. In Fig. 6-4 the hopper traverses the inner circle at $0.4 \mathrm{~m} / \mathrm{sec}$ while $\mathrm{D}_{\mathrm{Lat}}$ is maintained constant at $0.04 \mathrm{~m}$.

\subsubsection{Spiral trajectories}

Fig. 6-3 illustrates that the lateral acceleration during the stance phase increases with the lateral displacement of the foot, in the vicinity of the point of zero acceleration. If the distance $\mathrm{D}_{\mathrm{Lat}}$ is increased during each successive hop while the speed of the hopper is maintained constant then the lateral velocity imparted to the 
hopper during each stance phase also increases. The radius of curvature of the hopper's trajcctory is therefore constantly decreasing. The hopper therefore follows a trajectory that is close to a spiral. Fig. 6-5 shows the path followed by the hopper during such a maneuver.

Also if $\mathrm{D}_{\text {Lat }}$ is maintained constant while the speed of the hopper is constantly increased, the hopper would go outwards along a spiral trajectory. Fig. 6-6 shows the path followed by the hopper during such a maneuver.

\subsubsection{Square Path}

It is possible using asymmetrical foot placement to substantially alter the velocity of the hopper. This can be used to cause the hopper to follow a square path like the one in Fig. 6-1. While turning a corner the longitudinal velocity of the hopper has to be brought to zero and a lateral velocity has to be imparted. This is achieved by placing the foot forward and to the side of the point of zero acceleration. The exact foot piacement that is required to execute this maneuver is found by looking up a table as explained in section 6.2. Fig. 6-7 shows the path followed by the hopper while traversing a square with sides $3 \mathrm{~m}$ long. The path control strategy followed by the hopper causes it to turn after it has passed the corner. The turn is accomplished in a single hop without bringing the hopper to a stop in the horizontal direction. Deviations from the desired path are then corrected for by changing the direction of the hopper. Because the forward velocity is maintained constant at $0.7 \mathrm{~m} / \mathrm{sec}$ the step length is also fixed. Hence at the corners the hopper overshoots the desired path. The maximum overshoot is less than the step length of the hopper.

The entire path is traversed in $19 \mathrm{sec}$. This is a $47 \%$ decrease in traversal time compared to the method that stops and restarts motion at each corner. 
6.4 Appendix: Description of 3D Model

Dimensions of 3D one-legged model:

$\begin{array}{llllll}\text { Link } & \text { leg } & \text { cylinder } & \text { ring } & \text { body } & \text { Units } \\ \text { Description } & \text { Cylinder } & \text { Cylinder } & \text { Ring } & \text { Ring } & \\ \text { Mass } & .8626 & 0.5902 & 0.1 & 14.755 & \mathrm{Kg} \\ \text { Length } & 0.8 & 0.15 & 0.01 & & \text { Meters } \\ \text { Radius } & 0.01 & 0.02 & 0.03 & 0.3 & \text { Meters } \\ \text { Moments of Inertia: } & 0.0474 & 0.00674 & 0.00009 & 1.152 & \mathrm{Kg} \cdot \mathrm{m}^{2} \\ \mathrm{~J}_{\mathrm{xx}} & 0.0474 & 0.0004 & 0.000046 & 0.807 & \mathrm{Kg} \cdot \mathrm{m}^{2} \\ \mathrm{~J}_{\mathrm{yy}}^{\mathrm{yy}} & 0.0001 & 0.00674 & 0.000046 & 0.115 & \mathrm{Kg} \cdot \mathrm{m}^{2} \\ \mathrm{~J}_{\mathrm{zz}} & 0 & 0 & 0 & 0 & \mathrm{Kg} \cdot \mathrm{m}^{2} \\ \mathrm{~J}_{\mathrm{xy}} & 0 & 0 & 0 & 0 & \mathrm{Kg} \cdot \mathrm{m}^{2} \\ \mathrm{~J}_{\mathrm{yz}} & 0 & 0 & 0 & 0 & \mathrm{Kg} \cdot \mathrm{m}^{2} \\ \mathrm{~J}_{\mathrm{xz}} & 0 & 0 & & & \end{array}$

Denavit-Hartenberg description of the model:

$\begin{array}{llllll}\text { Joint \# } & \boldsymbol{\theta} & \mathrm{d} & \mathrm{a} & \boldsymbol{\alpha} & \text { Description } \\ 1 & \pi / 2 & \mathrm{Q}_{1} & 0 & \pi / 2 & \text { External degree of freedom. X displacement of foot. } \\ 2 & \pi / 2 & \mathbf{Q}_{2} & 0 & \pi / 2 & \text { External degree of freedom. Y displacement of foot } \\ 3 & \pi / 2 & \mathbf{Q}_{3} & 0 & \pi / 2 & \text { External degree of freedom. Z displacement of foot. } \\ 4 & \mathbf{Q}_{4} & 0 & 0 & \pi / 2 & \text { External degree of freedom. Orientation of leg. } \\ 5 & \mathbf{Q}_{5} & 0 & 0 & \pi / 2 & \text { Externai degree of freedom. Orientation of leg. } \\ 6 & \mathbf{Q}_{6} & 0 & 0 & 0 & \text { External degree of freedom. Orientation of leg. } \\ 7 & \pi / 2 & \mathrm{Q}_{7} & 0 & \pi / 2 & \text { Length of leg. } \\ 8 & \mathbf{Q}_{8} & 0 & 0 & \pi / 2 & \text { Orientation of leg with respect to hip. } \\ 9 & \mathbf{Q}_{9} & 0 & 0 & \pi / 2 & \text { Orientation of leg with respect to hip. }\end{array}$




\title{
7. Legged Locomotion Vignettes
}

\author{
Marc H. Raibert and Anthony J. Stentz
}

\subsection{Introduction}

In this chapter we collect together and discuss ideas about legged locomotion that have developed from our work over the past few years. Some of these ideas are preliminary to future work in our laboratory, while others may turn out to be half-baked or just plain wrong. All of the concepts discussed are characterized by a lack of experimental data or formal development. The purpose of collecting these discussions here is to provide an open repository for our developing thoughts where they can get some air and criticism, and perhaps lead to new ideas.
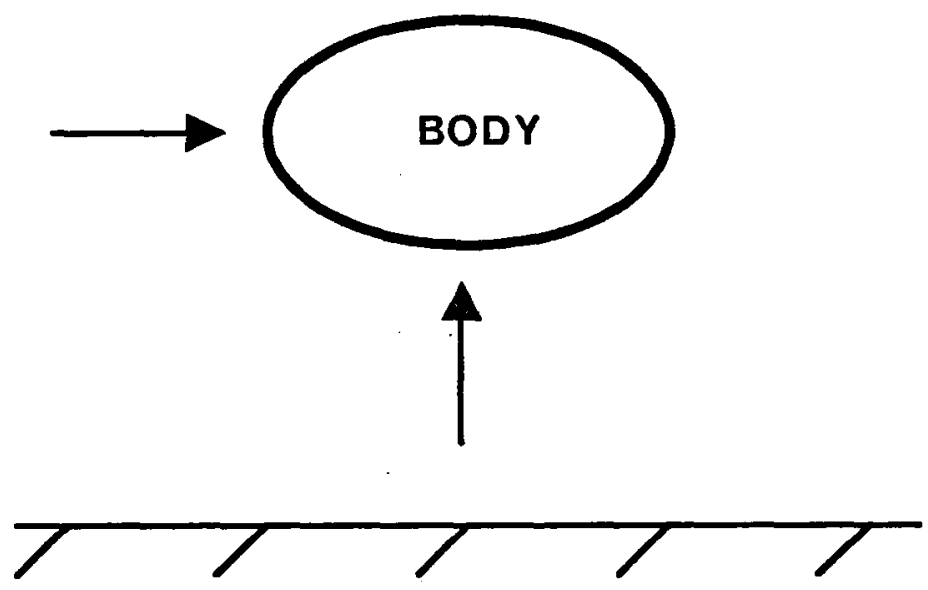

Figure 7-1: The purpose of legs in a legged system is to provide support for the body and to propel it forward.

\subsection{Locomotion Algorithms for $\mathbf{N}$ Legs}

It is now time to think about systems with several legs. We have argued that actively balanced legged systems with just one leg are easier to study and understand than those with many legs, and that the lessons learned from study of one-legged systems will generalize to multi-legged systems. How far can we generalize the ideas we already have for one-legged systems?

The discussion that follows is a first attempt to make such generalizations. The approach is to decompose the behavior commonly observed in biped and quadruped locomotion, into components that we know about from our work with one leg. We do not address the problem of gait selection, focussing instead on methods of maintaining balance and forward speed once a gait is chosen, if indeed it is correct to assume that gaits are chosen at all.

One way to generalize understanding of systems with one leg to systems with many legs, is to avoid focussing 

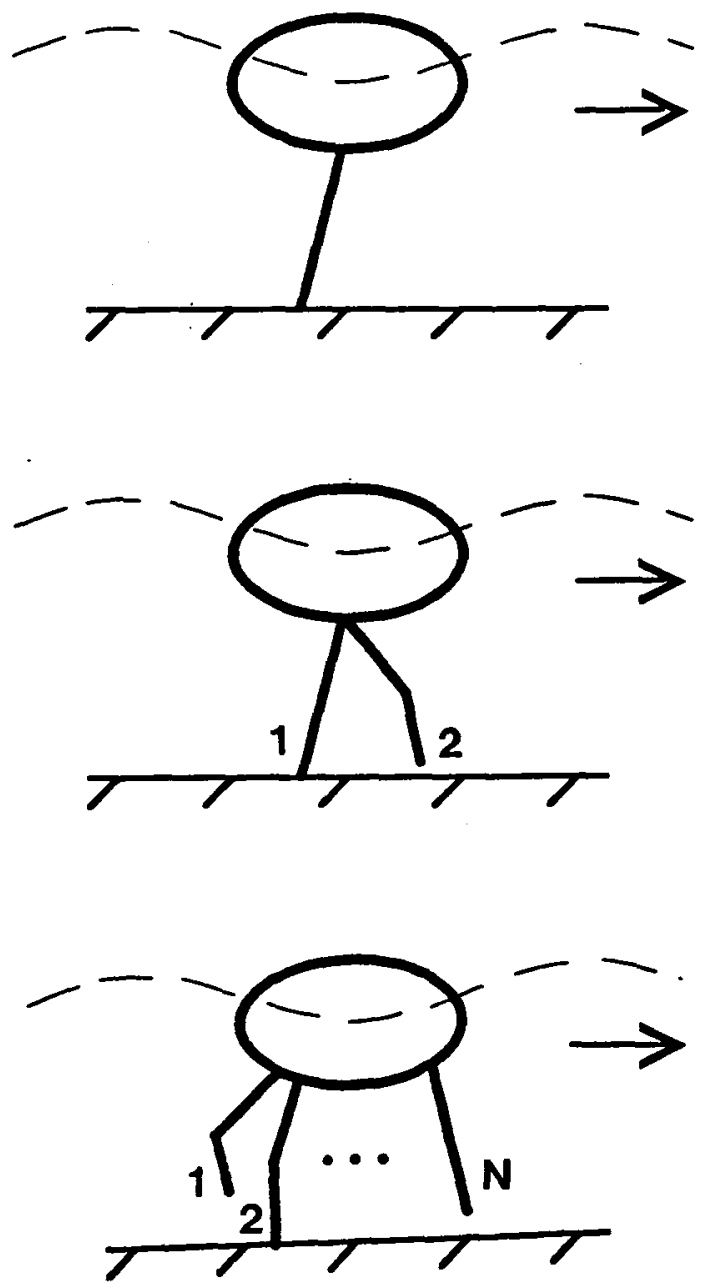

Figure 7-2: A legged system may run on 1 leg, 2 legs, . . N legs. We seek locomotion algorithms that are not tied closely to the number of legs.

on the legs themselves, and to concentrate, instead, on the influence the legs have upon motion of the body. The legs must provide support and they must propel the system forward. (See Fig. 7-1.) If we can understand what the body needs in terms of forces and torques to obtain support and propulsion, and if we can find ways to make the legs work together to provide support and propulsion, then we should be able to formulate effective control algorithms. Presumably, a control algorithm of this kind might apply to the locomotion of a system with one leg, two legs . . N legs. A theory that could explain dynamic behavior in all sorts of legged creatures, and that could guide the design of man-made vehicles is a primary goal of our research. 


\subsubsection{One Leg at a Time}

For the purpose of this discussion, let us take it for granted that our work on 2D and 3D systems with one leg provides effective algorithms for controlling devices that have a leg, a body, and a suitable collection of actuators and sensors. These algorithms involve providing leg thrust to maintain vertical hopping, placing the leg during flight to control the forward running velocity, and torquing the body during stance to control attitude. With this starting point, it is not hard to propose a mechanism that would work for humans and other bipeds running on two legs.

Despite a number of real differences ${ }^{1}$, running on one and two legs is remarkably similar. Rather than use one leg over and over again as a system with only one leg must, a biped alternates in the use of two separate legs. See Fig. 7-3. The thrust each leg delivers, the placement of the legs, and the torque generated between the hip and leg during stance can be identical to the corresponding values generated for systems with one leg. While one-legged systems use the same leg over and over to achieve these functions, the human biped performs these functions alternately, with the two legs. The basic characteristics as seen from the body are not much different in the two cases -- alternation of springy support and ballistic flight.

We characterize running by one- and two-legged systems in the following terms:

- Oniy one leg provides support at a time.

- Support phases and flight phases proceed in strict alternation.

We call this class of running gait the one-foot. All the legs are kept off the ground, except for the one whose turn it is to provide support. The human biped runs in this manner, as do the one-legged machines we have built. I know of no example of a natural quadruped that employs a one-foot gate.

In principle, the control algorithm used for the one-legged system will permit a systems with any number of legs to run using a one-foot gait. A quadruped performing the one-foot gait would cycle through use of the

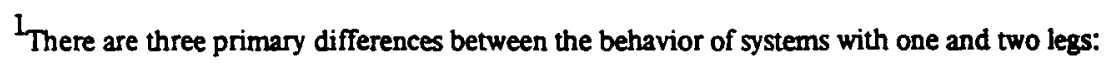

- Two legs permit running without pitching motions of the body. A one-legged system must move its leg forward and backward at different times. When a one-legged system swings its leg forward during flight. the body must pitch forward so that angular momentum is conserved. In a biped it is possible to overlap in time the backward motion of the supporting leg with the forward motion of the other leg. If the legs move forward and backward in this complementary fashion, then conservation of angular momentum during light can occur without pitching motions of the body, and without a tail.

- Since forward and backward motions of the legs can occur simultaneously in the biped, the time available for the recovery motion of the swing leg is not uniquely determined by the duration of flight. Therefore, if the recovery motion of one leg is overlapped with the stance motion of the other leg, then a biped will nun faster than a one-legged system if both can move a leg back and forth at the same rate.

- It is desirable for a biped to recover one leg to a forward position while the other leg supports weight. The recovery leg must be substantially shorter than the support leg. if it is to clear the ground without stubbing. In the one-legged systems we have explored. the leg was always at maximum length during recovery, but the forward recovery motion occurred when the body achieved peak height during flight. A biped should have a mechanism that will permit the swing leg to shorten substantially during recovery, and to lengthen again in time for landing. 

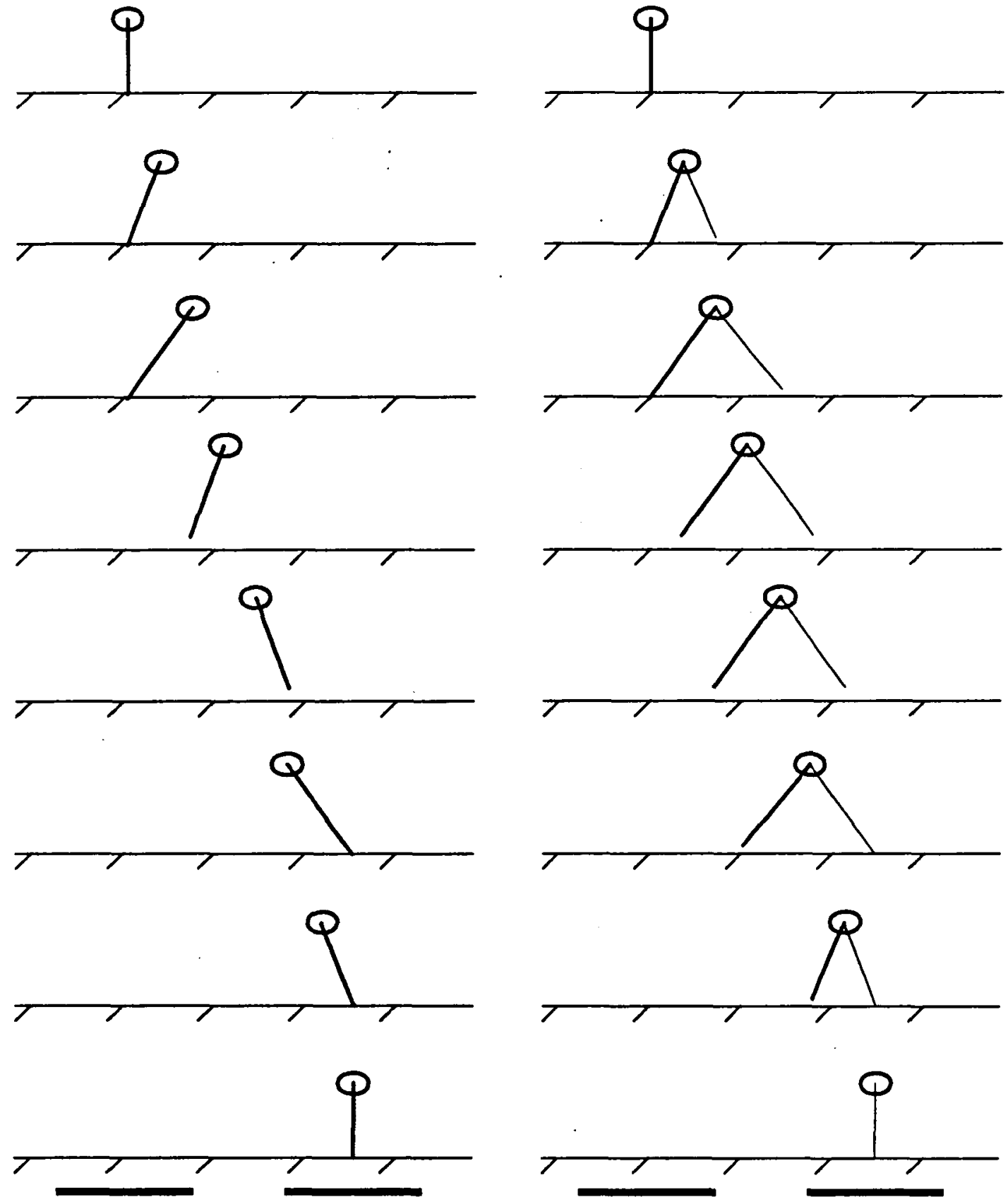

Figure 7.3: Running on two legs, (right), is very much like nunning on one leg, (left). Rather than using one leg over and over by recovering immediately at the end of the stance phase, the two legs alternate. Recovery of one leg is overlapped with the stance phase of the other. The horizontal bars at the bottom indicate CG-prints. 
legs in some regular order. Each would deliver a vertical thrust to maintain hopping and correct body attitude during stance when providing support, while the next leg moved to a forward position appropriate for landing. It would be like a Gatling gun with legs in place of barrels.

A practical problem with this sort of running is the difficulty of locating the legs close enough to the center of the body to permit the feet to reach the points that would provide balance. The foot must be placed so that the average point of support during stance is under the center of mass. It is not hard to attach one or two legs near the center of mass, but the design problem becomes more difficult with more legs. It is also hard to keep many legs from interfering with each other in their motions, when they are mounted close together.

To summarize, the one-foot is a class of running gaits in which only one leg provides support at a time and a flight phase occurs between each support phase. Control of each leg in a system executing a one-foot could be like that used in our one-legged systems.

\subsubsection{Pairs of Legs in Unison}

The next step in generalizing from one leg to many legs is to consider pairs of legs that act together. Quadruped gaits that might be understood and produced in this way are the trot, the pace or rack, and the bound. In each of these gaits, two legs strike the ground in unison, and they leave the ground in unison. While one pair of legs is providing support, the other pair swings forward for the next step.

Suppose for the moment that when a pair of legs provides support, both members of the pair are controlled to act like an equivalent single leg. This is Sutherland's idea of a virtual leg (Sutherland, 1984). One might suppose that the effective point of support is determined by the position of each foot and the force each delivers to the ground. The sum of torques delivered by the pair of hip actuators would contribute to the hip torque, but the difference in thrust of the legs would also influence the effective torque. The height of a hop would be determined by the sum of thrusts. Chapter 3 of this report explores the problem of controlling pairs of legs to make them act together in these ways.

Thinking in terms of pairs of legs, the trot, the pace and the bound are very much like a biped one-foot, in which each biped leg is replaced with a pair of legs. Given that pairs of legs can be controlled to act like single virtual legs, then these gaits reduce to that of the running biped, which we have already discussed and reduced further. The kangaroo ricochet reduces to a one-legged system directly.

An important difficulty in realizing the one-foot running gait with a quadruped is the difficulty of locating the legs close enough to the system's center of mass. When pairs of legs act together during support, the effective point of support can be located near the center of mass, even though the physical legs are located a substantial distance from the center of the body. When two legs provide support simultaneously, the effective point of support lies somewhere on the line that connects the two feet. In a trot, the gait involving diagonal support pairs, the lines containing the effective points of support for both leg pairs pass under the center of the body. In the pace, the gait involving lateral support pairs, the lines connecting the feet may pass under the center of 


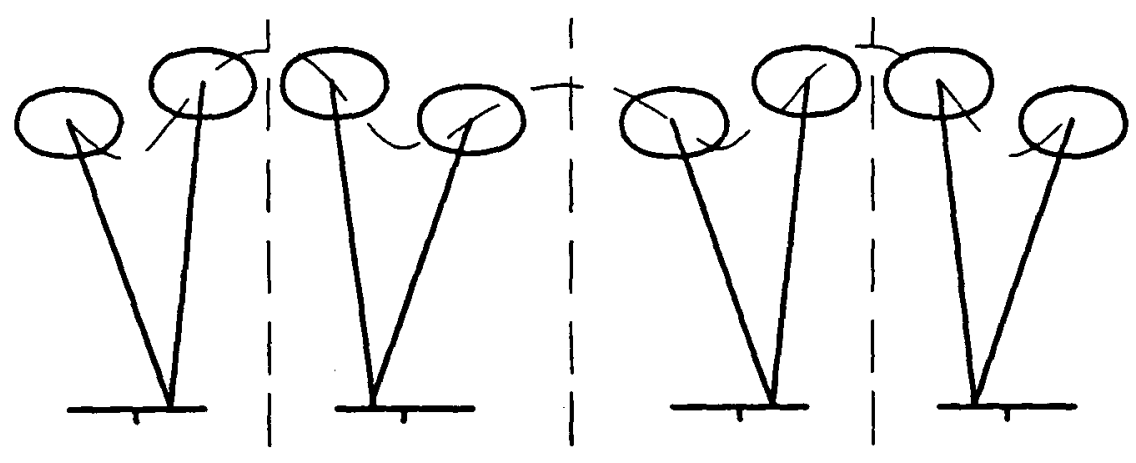

Figure 7-4: Sequence in which body and leg are shown at each touch-down and lift-off, for pairs of steps that are symmetrical. If the foot is positioned behind the center of the CG-print on one step, and in front of the center on the next step, then there can be a symmetrical pattern that produces zero net acceleration over the pair of steps. The strides will be of different sizes because the speed during the flight phase of each step is different. The dashed lines indicate symmetry.

mass if the legs are angled inward during stance, or they may pass quite close to the center of the body if the body is narrow.

The bound is another gait that employs pairs of legs acting in unison, but the analysis does not account for stability in that case. For quadrupeds with long bodies, the lines connecting supporting feet do not pass near the center of mass. Some other mechanism must be at work to provide stability. See Chapter 3.

\subsubsection{Pairs of Legs in Sequence}

A basic tenant of dynamic stability is that the legs need not provide a base of support continuously, but only over time. The stability we have described so far in this discussion relies on finding a foot placement that will result in symmetrical behavior during each support interval, for each leg or pair of legs. If the motion of the body is symmetrical about the effective point of support provided by the feet then there is no net acceleration of the system. We now consider the case where each support interval causes the system to accelerate, but successive pairs of support intervals are matched to generate equal and opposite accelerations. See Fig. 7-4. Such anti-symmetric pairs of steps generate no net acceleration.

Suppose we modify the control algorithm for the one-legged system, so that on every even hop the algorithm that calculates desired foot placement adds in an extra factor, $\Delta x$, and on every odd hop it adds an extra factor, $-\Delta x$. For some suitably small range of $\Delta x$, the system would hop side to side, with no net horizontal acceleration. Figure 7-5 plots data from just such an experiment, performed with the $3 \mathrm{D}$ one-legged hopping machine.

The system continues to balance provided that the accelerations caused by the offset of the foot do not cause the system to tip over entirely before the next step. On the next step there will be an opportunity to accelerate 


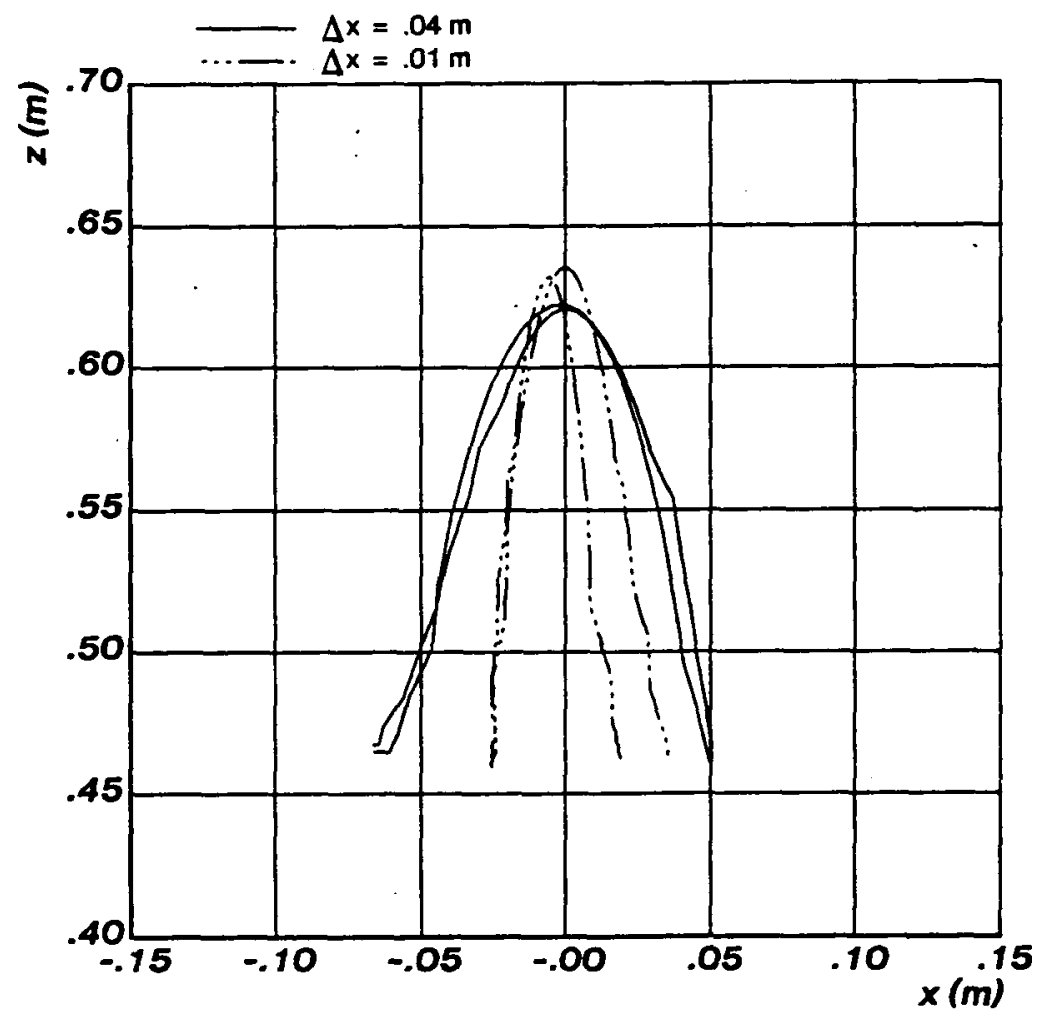

Figure 7-5: Position of center of mass of physical 3D hopping machine using pairs of balanced steps. The control algorithms were those described in Chapter 2, but an offset, $\Delta x$, was added to the foot position on even hops, and suburacted on odd hops. The magnitude of $\Delta x$ is set to two different values. The body moved back and forth on alternate hops. The system was not stable during a single hop, but only over a pair of hops. This experiment is relevant to running gaits that do not place the effective point of support under the body, such as the pace and bound. The displacements of the foot, $\pm x$, correspond to the displacement of the hip from the center of the body. The trot and kangaroo hop do not involve such offsets.

the system in the other direction, with no net acceleration over the pair. This analysis becomes relevant when we think of $\Delta x$ as representing the distance from the hip to the center of the body. For the pace, $\Delta x$ may be small. For the bound $\Delta \mathrm{x}$ is about half the length of the quadruped.

\subsubsection{Pairs of Legs Overlapped in Time}

The most complicated quadruped running gaits, the canter, the gallop and the half bound, do not yet fit into this framework. These gaits are characterized by partially overlapping periods of support between pairs of legs and larger numbers of legs. For instance, in the gallop the following sequence for the front feet is typical: place front right, place front left, lift front right, lift front left. See Fig. 7-6. Similar overlapping support periods occur for other pairs of legs. 

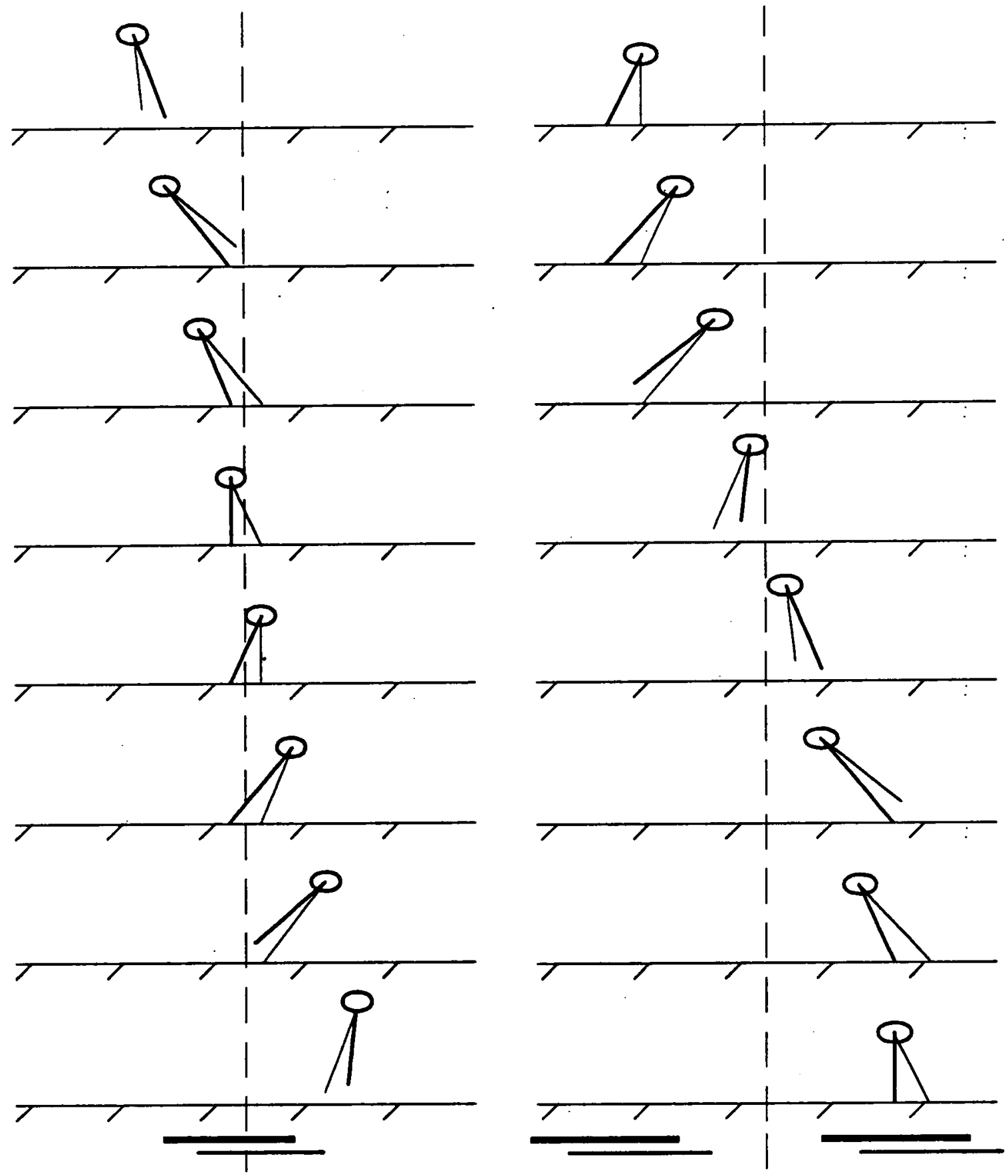

Figure 7-6: Galloping with two legs. In a gallop the two legs are just slightly out of phase. One leg touches down and lifts off just ahead of the other. There can be symmetry in a gallop, about both the center of stance, when both legs are touching the ground and equally compressed (left). and about the half way point in flight, (right). The dashed lines indicate the symmetry planes. The horizontal bars at the bottom indicate the CG-prints for each leg. See also, Fig. 7-7 


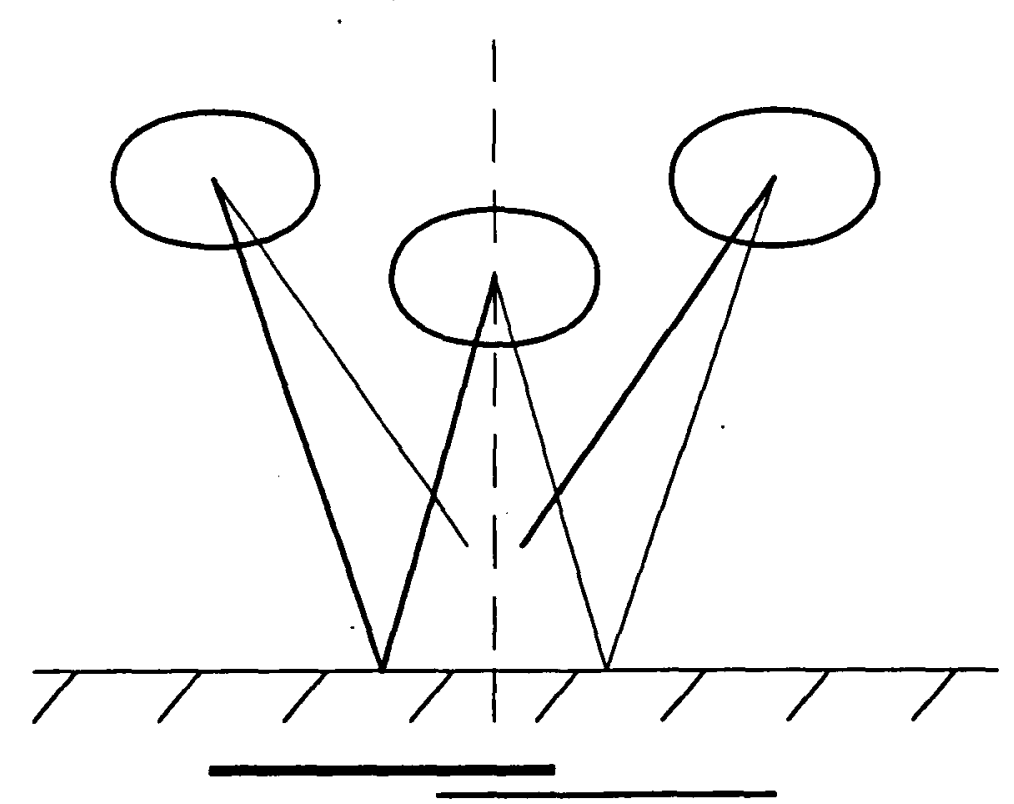

Figure 7-7: Symmetry that exists about the center of the stance phase in a gallop, for a pair of legs. The bars underneath the figure indicate the CG-prints for each leg.

So far we only have a vague idea of how to decompose and understand these sorts of gaits. To simplify the analysis, consider a biped running with overlapping support periods for each leg. Humans can gallop in this way. See Fig. 7-6. Assume that both legs have equal support periods, and therefore, the CG-print associated with both legs are of equal length. We see that in steady state running, the biped with overlapped support still has a symmetrical support pattern. The single support phases at the beginning and end of the stance interval have symmetry about the center of the stance interval. The configuration of the body and legs during the double support phase is also symmetrical.

\subsubsection{Independent Legs}

So far we have concentrated on representing the behavior of entire multi-legged systems in terms of the equivalent behavior of one-legged systems. Another way to generalize the results from the one-legged case to the multi-legged case, is to think in terms of the behavior of several one-legged systems that are loosely constrained to operate together. The idea is that each leg of a multi-legged system, along with part of the body behaves like the one-legged systems we have described. Perhaps during a gallop, the vertical bouncing motion of the front half of a horse might be controlled separately from the vertical bouncing motion of the back half. This approach is explored more fully in Chapters 3 and 5 of this report.

A primary motivation for our work on systems with one leg was to provide simple ideas about legged locomotion that we could generalize to more complicated configurations with more legs. Actually, we would like to find concepts that are not tied to particular locomotion systems, but that apply to whole classes of 
legged systems. We would like to transform the question of how many legs a legged system has, from a central determinant of behavior to a mere implementation detail.

To summarize briefly the main points of this section:

- Existing algorithms are effective in controlling systems with a body and one springy leg. They decompose control into a hopping part, a forward velocity part, and a body attitude part.

- Bipedal running is very much like running on one leg, except that two legs are used in alternation. The same control algorithms apply.

- There is a special class of gaits we call the one-foot, for which only one leg touches the ground at a time. The same control algorithm applies to all one-foot gaits, independent of the number of legs.

- When pairs of legs acting in unison are controlled to behave like single virtual legs (Sutherland, 1984), then the trot and pace of a quadruped are like the biped run.

- The quadruped bound is also like a biped run, but with an alternating offset in the point of support.

- We do not yet understand the canter, gallop, or half bound, though there is symmetry in the pattern of stepping for these multi-legged gaits.

\subsection{Scissor Symmetry}

When a human runs, the two legs form symmetric angles with respect to the vertical axis. The angle formed between the hip and foot of the forward leg and the vertical axis, is equal and opposite to the corresponding angle for the rearward leg. See Fig. 7-8. This symmetry is largely independent of the speed, bounce, stride, and other parameters of the gait. The behavior reminds one of the way the blades of a scissors orient themselves with respect to the paper they cut.

The reader can observe this scissor-like motion by doing an informal experiment. Run forward at an even, moderate pace. During one stride take a larger step than normal, reaching out with the swing leg. As the swing leg thrusts forward, the stance leg will stretch backward, maintaining the symmetry of the legs about the vertical axis and keeping the body in an upright posture. Most normal walking and running activities preserve this approximate symmetry of leg motion.

A consequence of the scissor symmetry is that during flight, the angle of the leg to be placed is about equal to the angle of the leg that was just lifted. Fig. 7-8. Can we use this symmetry to formulate an algorithm that can correctly place the foot? It seems that the nominal motion produced by a scissor algorithm can be very similar to the motion produced by the CG-print algorithm that we used for the one-legged systems (Raibert, 1984; Raibert and Brown, 1984). However, there is a stability problem that we have not yet solved.

The scissor algorithm we have in mind specifies that during flight the next leg be positioned to the negative of the angle the previous leg had when it left the ground: 

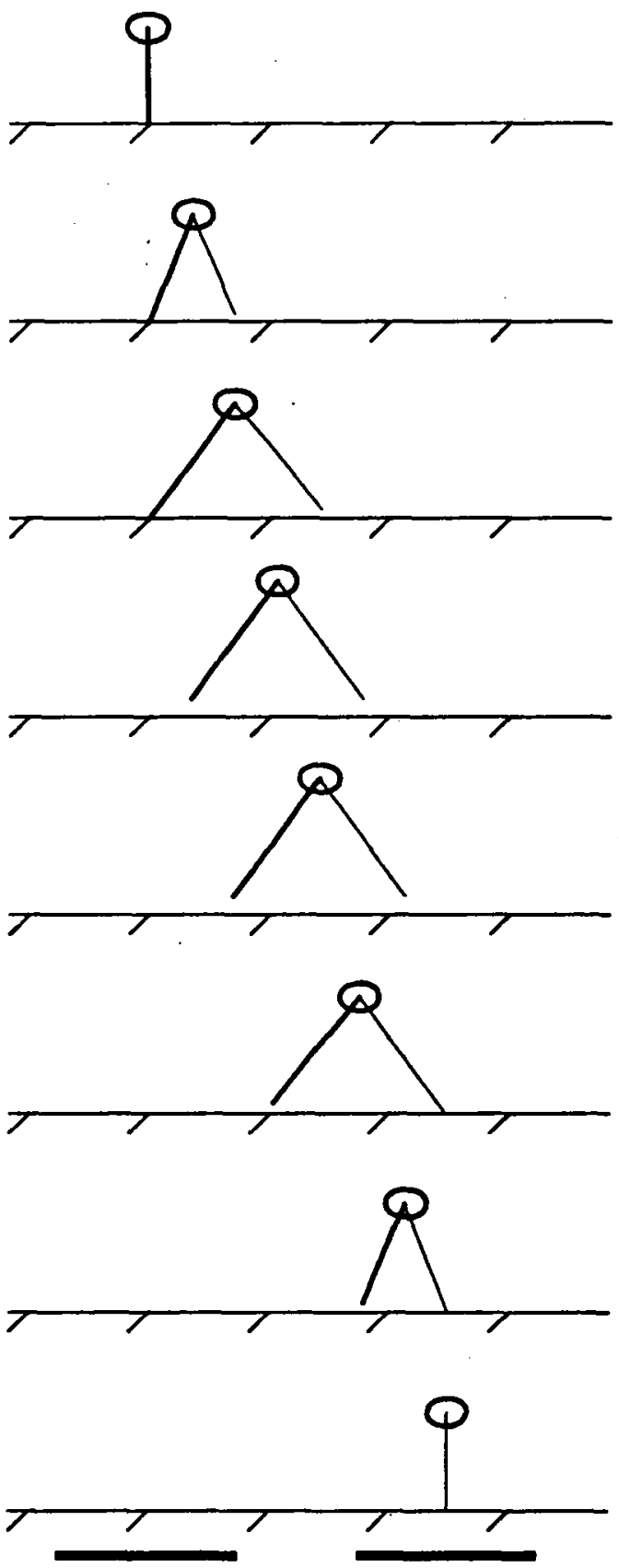

Figure 7-8: Diagram that shows how normal action of the legs on successive steps may behave like a pair of scissors. The further back one leg is when it leaves the ground, the further forward the next leg should be when it is touches the ground. Bars at bottom indicate CG-prints for each leg. 
$\theta_{\mathrm{d}}=-\theta_{\mathrm{LO}}$

where

$\theta_{d} \quad$ desired angle of leg with respect to the vertical, and

$\theta_{\text {L }} \quad$ angle of leg when foot last left the ground.

This could be used for any number of legs. What happens when the scissor algorithm is used for a series of steps? For a biped with legs $A$ and $B, \theta_{A}(t)=-\theta_{B}(t)$, for all $t$. Let us assume for the moment that the duration of stance is constant, and that the body moves forward during stance at a roughly uniform rate. If $\operatorname{leg} A$ is placed forward of the center of the CG-print with angle $\theta_{A, T D}$, then the lift-off angle of that leg, $\theta_{A, 1}$ ' will be less than it was at touch-down, $\left|\theta_{A, L O}\right|<\left|\theta_{A, T D}\right|$. Leg B is then placed according to the scissor symmetry: $\theta_{\mathrm{BTD}}=-\theta_{\mathrm{A}, \mathrm{D}}$. This results in placement of leg B's foot behind the center of the CG-print. However, when leg $B$ leaves the ground its angle will be larger than it was at touch-down, $\left|\theta_{B, L O}\right|>\left|\theta_{B, T D}\right|$. Moreover, the angle will have returned to the original touch-down angle of leg $A, \theta_{B . L O}=-\theta_{A, T D}$. The result is that the system repeats its behavior after every pair of steps. Fig. 7-4. This pair of steps has a symmetry that can maintain balance in the system.

This same mechanism will work properly during forward accelerations. Suppose that during stance an external disturbance accelerates the system forward. The result is that the stance leg sweeps further back and the lift-off angle of the stance leg is larger than it would have been without the disturbance. The other leg is placed correspondingly further forward, compensating for the increased velocity. A decelerating disturbance works in a corresponding manner. The acceleration need not be due to an external disturbance, but could be caused by actions of the hip actuator, intended to stabilize the body attitude or to regulate forward speed. They might even be caused by the actions of other legs in a more complicated system.

One way to look at the scissor algorithm is that it provides an alternate method for estimating the length of the CG-print. The lift-off angle of the stance leg serves to indicate the system's forward velocity and ground time. The faster the body moves forward relative to the ground, the further backward moves the foot during stance. The foot also moves backward further when the system spends more time on the ground. Therefore, the angle of the leg at lift-off is determined by the product of the average forward velocity and the duration of stance.

$$
\theta_{10}=\operatorname{Arcsin}\left[\frac{\dot{x} T_{S I}+w_{L} \operatorname{Sin} \theta_{L D}}{R}\right]
$$

The angle of the leg at lift-off is also influenced by the angle of the leg at touch-down. The product of average velocity and ground time provides only the change of leg angle, so the starting angle of the leg at touch-down will determine where it ends up.

When the leg is positioned in the center of the CG-print at touch-down, and when there is constant average forward velocity, then touch-down and lift-off leg angles for a single stance period are cqual and opposite. 
Under these circumstances, all steps are identical. When the foot is placed a distance $\Delta \mathrm{x}$ away from the center of the CG-print and there is a constant average forward velocity, then every other pair of steps is the same, but steps that are adjacent in time are mirror images of one another. The positions of the foot with respect to the hip at touch-down and lift-off are skewed forward or backward by $\Delta \mathrm{x}$ on alternate steps. Fig. 7-4. Such a pattern of paired anti-symmetric steps will provide balance despite the foot offset, provided the degree of asymmetry is relatively small and the step rate is large.

There are two problems with the scissor algorithm as presented here. First, while in principle the algorithm is able to generate a sequence of uniform symmetrical steps, in practice there is no mechanism to keep the algorithm from drifting from a symmetrical stepping pattern to anti-symmetrical pairs of skewed steps. For example, during simple hopping in place successive hops might carry the leg into pairs of more and more skewed anti-symmetrical pairs. This problem might be overcome by somehow damping the foot placement excursions, or perhaps by using information from previous steps to filter the two-step oscillations. Another alternative might be to take both the touch-down and lift-off angles into account:

$$
\theta_{\mathrm{d}}=\frac{\theta_{\text {To }}-\theta_{10}}{2}
$$

$$
\text { where }
$$

$\theta_{d} \quad$ desired angle of leg with respect to the vertical,

$\theta_{w} \quad$ angle of leg when foot left the ground last time, and

$\theta_{\text {TD }} \quad$ angle of leg when foot touched the ground last time.

A second problem with the scissor algorithm is that it may not be responsive to sudden changes in the forward velocity of the body. The algorithm is based on the idea that the lift-off angle of the leg gives information about the forward velocity of the system. Actually, the information provided is about the forward velocity averaged over the entire previous stance interval. In some cases the effective latency of this measurement could result in sluggish corrective actions, and instability.

How do the motions produced by the scissor algorithm compare to those produced by the CG-print algorithm? Consider the case where the foot is placed in the center of the CG-print, and the length of the CG-print equals the duration of stance times the average forward velocity. For a multi-legged system, there is a CG-print associated with each foot. We assume a constant ground time and constant forward velocity. Under these circumstances, the displacement of the hip during stance is such that the angle of the stance leg at lift-off is the same as it was at touch-down. Each step is identical, with equal touch-down and lift-off leg angles. Therefore, for constant speed locomotion with no disturbances, and an appropriate starting configuration, the scissor algorithm and the CG-print algorithm produce the same motion. Since it is hard to estimate the length of the CG-print accurately, the scissor algorithm is attractive. It avoids the need to measure the forward velocity of the system and the duration of stance.

To summarize briefly: 
- For any forward angle of the touch-down leg, a scissor algorithm will generate a pair symmetric steps.

- If the forward leg angle chosen by the scissor algorithm happens to put the foot in the center of CG-print, then each step of the pair will be the same length.

- The scissor algorithm is really just a simpler and more direct way to measure the forward velocity and the duration of stance.

\subsection{Why are Human Feet Long?}

The one-legged systems we have built used locomotion algorithms that control forward velocity through placement of the foot. In particular, the control algorithm first calculates a forward position for the leg based exclusively on the expected CG-print, the locus of points over which the system's center of gravity is expected to travel during the next stance phase. Then the control algorithm modifies the leg position according to the error in forward velocity. The equation for determining the desired foot position is (Raibert and Brown, 1984):

$$
\begin{aligned}
& \Delta x=\frac{\dot{x} T_{S T}}{2}+K\left(\dot{x}-\dot{x}_{d}\right) \\
& \text { where } \\
& \Delta \mathrm{x} \quad \text { is the forward displacement of the foot with respect to the projection } \\
& \text { of the center of gravity, } \\
& \dot{x} \quad \text { is the forward velocity, } \\
& \dot{x}_{d} \quad \text { is the desired forward velocity, } \\
& \mathrm{T}_{\text {ST }} \quad \text { is the duration of a support period, and } \\
& \mathrm{K} \text { is a feedback gain. }
\end{aligned}
$$

This method of velocity control is based on the idea that the system will undergo a net forward acceleration during a single period of support, if the center of mass spends more time in front of the point of support than behind it. When the controller wants to accelerate the system forward, the foot is displaced backward, to increase the time the body spends in front of the foot. Backward acceleration is produced by moving the foot forward. This method derives from our understanding of the inverted pendulum, and the symmetry of forces and moments that develops during stance (Raibert, 1984). For a given forward velocity, placement of the foot will determine the net forward acceleration of the system during stance.

Must the control system actively calculate $\mathrm{K}\left(\dot{\mathrm{x}}-\dot{\mathrm{x}}_{\mathrm{d}}\right)$ to correct for errors in forward velocity, or could a long foot, one extended in the direction of running, correct velocity errors by purely passive means? We have concluded that an extended foot might perform the desired correction, but only if the length of the foot is comparable to the length of the CG-print. 


\subsubsection{Circle Feet}
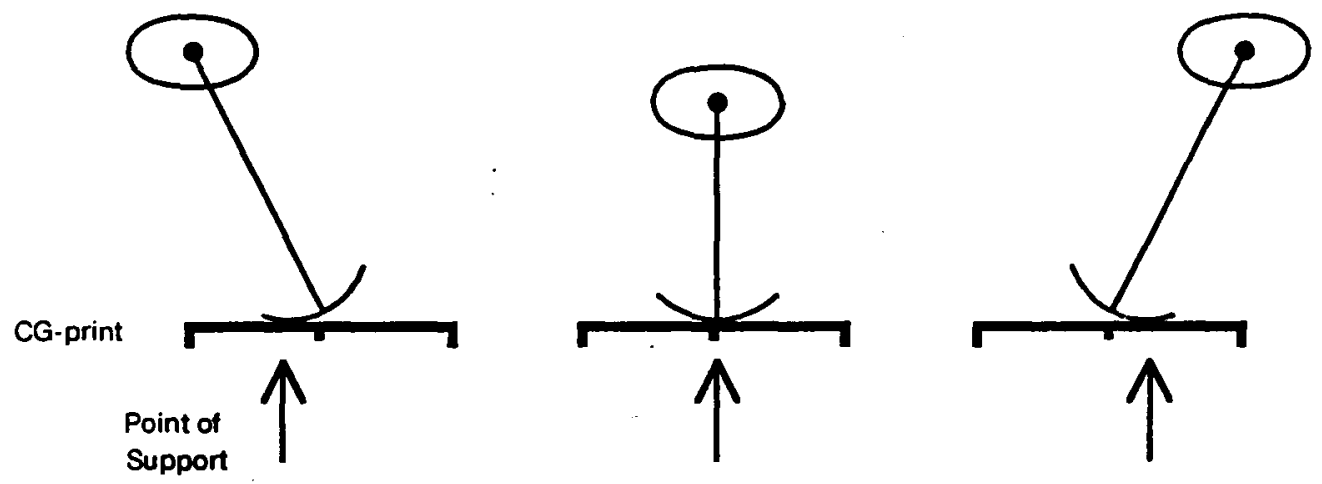

Figure 7.9: The instantaneous point of support provided by an extended foot that forms an arc of a circle, is determined by the angle of the leg with respect to the vertical and the radius of curvature of the foot. As the body moves forward, so does the point of support. Does the point of support move in a way that provides velocity stability when there are velocity errors? See discussion in text.

The starting point for these thoughts can be understood in terms of Fig. 7-9. Assume that on each step the system spends a fixed time with the foot touching the ground, $T_{\text {ST, }}$, and that during the time the foot is on the ground the body moves forward with a roughly uniform velocity. When the forward velocity corresponds to the actual velocity, then placing the foot in the center of the CG-print causes the leg to sweep through a pattern that has left-right symmetry during stance. The angle of the leg to the vertical at touch-down equals the angle of the leg at lift-off, and the pattern of intervening leg angles is also symmetric.

The average point of support provided by a curved foot under these circumstances would fall on the center of the CG-print. If the system were going faster or slower than expected, then the pattern of leg angles during stance would be skewed to one side or the other, moving the average point of support away from the center of the CG-print. Slower body travel would move the average point of support backward and faster travel would move it forward. A circular foot would provide displacements of the point of support with the same sign as those generated by Eq. (7.4). However, the magnitude of the corrections would be inadequate to provide stability, for practical size feet.

First consider a wheel and axle that support a load. Figure $7-10$. When the axle passes through the center of the wheel, the wheel has no preferred orientation on the ground. When the axle passes through a point other than the center, then the wheel will seek equilibrium at the point on the rim closest to the axle. There are two factors that describe changes in the stability of the system for variations of the point at which the axle passes through the wheel. As this point moves on a straight line from a point on the rim, A, through the center of the wheel, to an opposite point on the rim (see Fig. 7-10:

- The preferred point of support on the rim changes abruptly from one side to the other as the axle location moves through the center. 


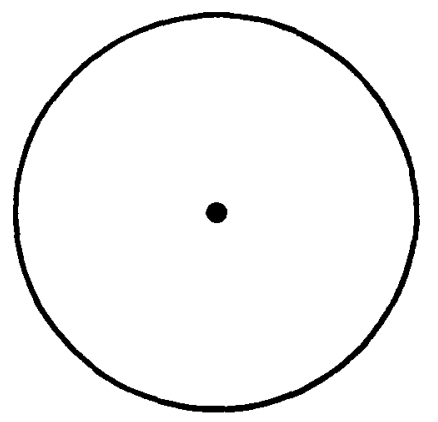

A

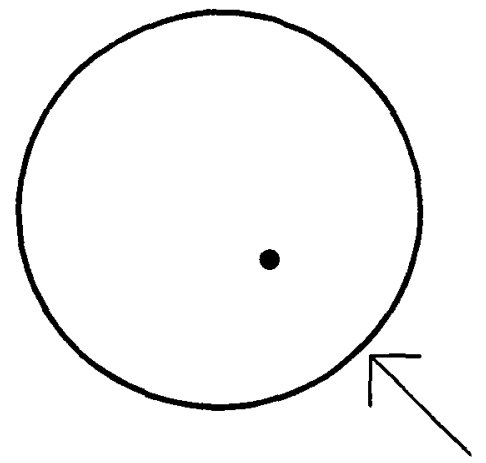

B

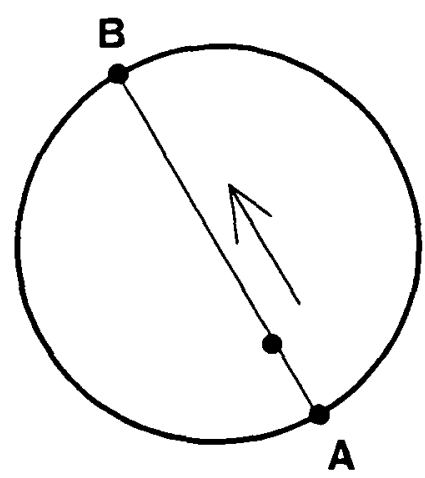

C

Figure 7-10: Wheels. LEFT: A normal wheel has no preferred point of support. MIDDLE: If the axle does not go through the center of the wheel, then the point on the rim closest to the axle is the preferred support point. RIGHT: As the location of the axle moves from point A to point $B$, the degree of stability goes through a minimum, and the location of the preferred point changes abruptly from $A$ to $B$.

- The degree to which the system is stable with point A touching the ground changes monotonically with the ratio: (distance between the axle axis and $A) /($ radius of wheel).

If we use a section of a wheel for a foot, then the radius of curvature of the foot and the length of the leg will determine to what extent the foot improves stability. See Fig. 7-11. The gravitational tipping moment on an inverted pendulum is related to the horizontal separation between the center of mass and the point of support. For a leg of length $w_{L}$, a circular foot with a radius of curvature $r$, and an angle between the leg and the vertical, $\theta$, the horizontal separation between the center of mass and the point of support is:

$$
\Delta x=\left(w_{L}-r\right) \sin (\theta)
$$

The system is least stable when the foot is a point, $r=0$, neutrally stable when $r=w_{L}$, and maximally stable when the foot is flat, $r=$ infinite.

The difficulty with this result is that the effective radius of curvature of the foot is limited by the arc length of the foot $l$. When the leg angle is greater than the foot angle, $\alpha$ in Fig. 7-11, then the point of support no longer changes with increasing leg angle, so the effective radius of curvature of the foot zero. Therefore, a circular foot with a large radius of curvature is equivalent to a foot with smaller radius of curvature, when the support phase includes leg angles greater than $V r$.

To summarize:

- A point foot provides no stability.

- A foot with a smaller radius than the leg length is still unstable, but the destabilizing forces are smaller, for the same deviation from unstable equilibrium.

- A foot with a larger radius than the leg length will provide stability.

- The effective radius of curvature of a foot is limited by the length of the foot. It decreases when the leg angle is greater than the ratio of foot length to leg length, $\theta>V r$. 


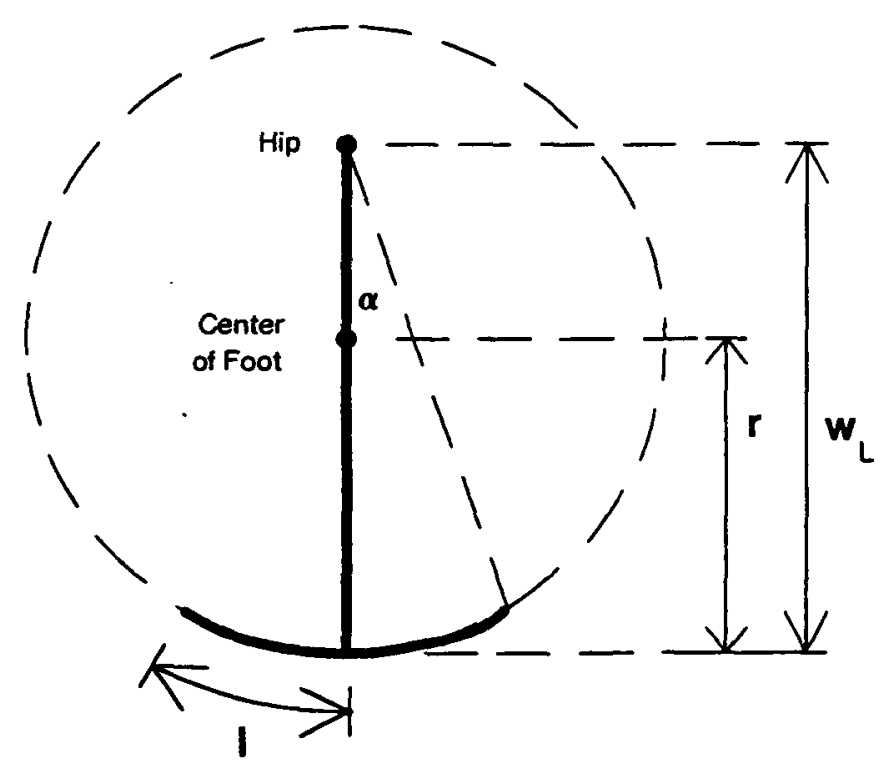

Figure 7-11: The foot can be an arc of a circle of radius, $r$. The angle subtended by the foot is $\alpha$.

Another way to build a foot that has some of the characteristics of the circle foot, is to use a hinged member with a centering spring that torques the foot toward a neutral position. The nature of the spring will determine the exact characteristics of the stability function. However, the upper limit of the stability provided by such a device is still governed by the length of the foot. As the spring becomes stiffer and stiffer, this arrangement approaches the equivalent circular foot with an infinite radius of curvature. It cannot provide greater stability.

The limitations of these passive schemes do not argue against the possibility of providing stability with an actively driven ankle.

\subsubsection{Yaw Control}

The discussion of a long foot has focused so far on the potential use of feet as a means of providing balance and of controlling forward velocity in a simple way. Another reason for a long foot might be to provide traction about the yaw axis. Quadrupeds and the hopping bipeds usually have more than one foot on the ground at a time, so generating torques that stabilize motion about the yaw axis is not a big problem. Pairs of horizontal forces can be used to generate yaw torque. But human running only entails single support. Therefore each foot must have sufficient torsional traction to stabilize yaw orientation. 


\subsection{Foot Placement for Leaping}

The algorithms that we have studied in the context of one-legged systems do a reasonably good job of controlling the forward running velocity, but they do not provide a direct means for controlling precisely where the foot is placed on a particular step. Precise foot placement is important if a legged system is to travel in rough terrain. It is important where much of the terrain will not provide adequate support or where there are obstacles. In these cases, the ability to place the foot precisely will permit the legged system to choose a good foothold among many possible places to put the foot, or to approach an obstacle in a way that allows the system to leap over it. See Fig. 7-12.

There are two classes of foot placement task. In one case, there is a single desired point on which to place the foot, and several steps are available in which to make the necessary adjustments. Humans encounter this sort of task when they jump a fence or ditch. The other case requires the foot to be placed in particular locations on a number of consecutive steps. Using stepping stones to cross a stream is an example of this sort of task.

Of course, there is nothing stopping a control system from altering the leg angle during flight so that the foot lands on any desired spot. The trick is to do this in a way that does not cause the system to tip over. In the context of the control algorithms that we have already explored for one-legged systems, four strategies come to mind for controlling foot placement while maintaining balance:

- Adiust hopping height: With forward velocity held constant, hopping height detcrmines the distance traveled during flight. The magnitude of thrust delivered by the leg could be adjusted on each step to determine the location of the next foothold. The forward velocity control algorithm could operate unchanged.

- Adjust leg stiffness: The distance the body travels during stance, the length of the CG-print, is the product of the average forward velocity and the duration of stance. The duration of stance is determined by the mass of the body and the stiffness of the leg. The control system can control the length of the CG-print, and thereby influence where the foot will be placed on the next step, by modulating the stiffness of the leg. This manipulation need not affect the forward velocity.

- Adjust forward velocity: For a given stance duration and hopping height, the forward velocity determines the stride length. The control system can manipulate forward velocity to place the foot.

- Direct placement: For a given leg stiffness and forward velocity, there is a particular location on which the foot should be placed to keep the system balanced. The foot may be moved from this location if corrective action is taken on subsequent steps. If the foot is displaced a distance $\Delta \mathbf{x}$ from nominal on one step, then it can be displaced a distance $-\Delta x$ on the next step. $\Delta x$ must be small enough so that the system does not tip over entirely before the next step can occur.

The direct placement method only produces a temporary change in the pattern of footholds. After the two modified steps, subsequent footholds are located as they would have been had no mancuver been made. The velocity method has the disadvantage that it requires forward velocity to change before placement changes. It is our experience that several steps are required to change the forward velocity appreciably. This same problem also exists for the height method in a system that cannot modify hopping height in one bounce. We 

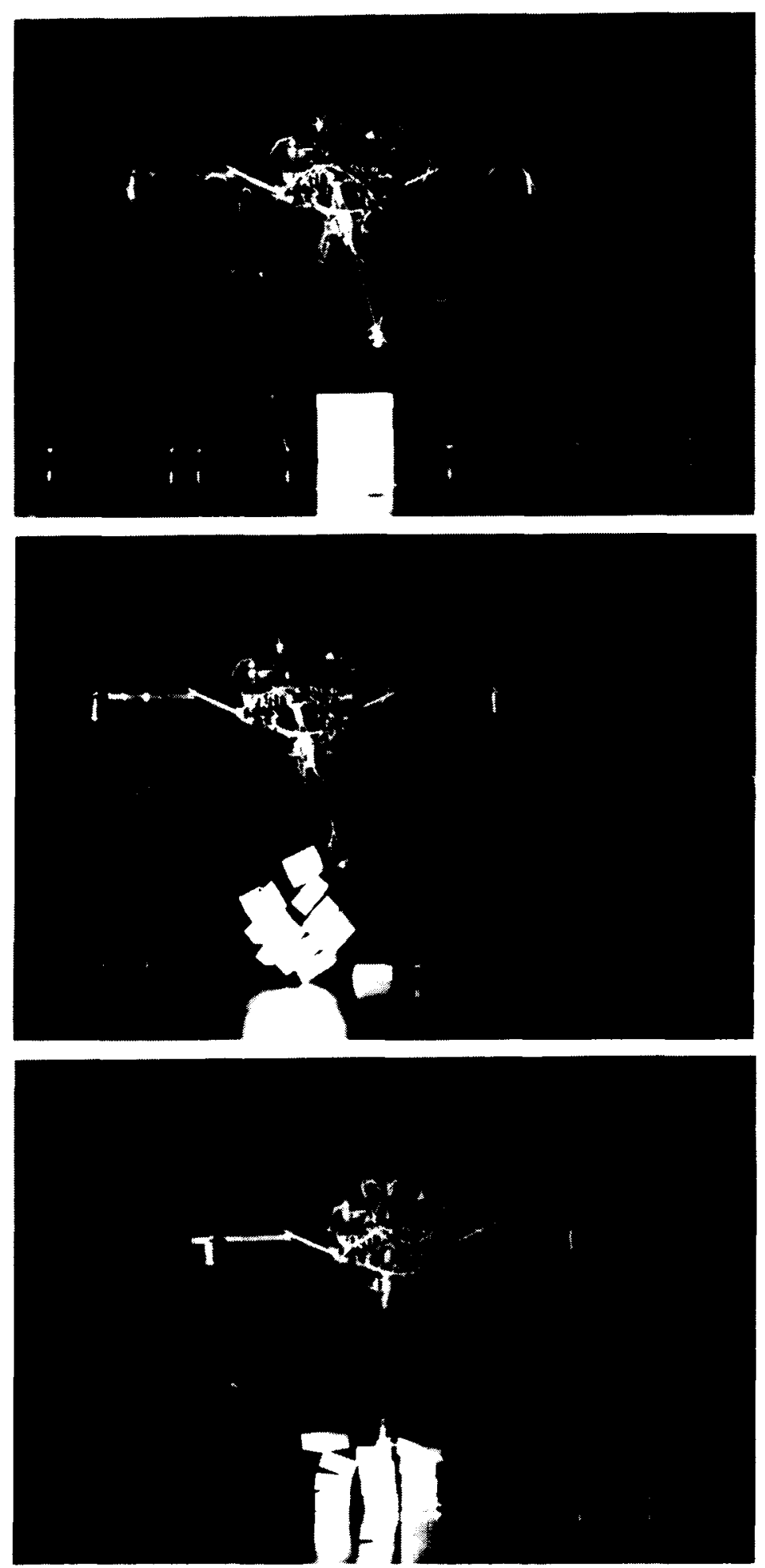

Figure 7.12: Planar one-legged system leaping. In order to successfully leap over an obstacle, a legged system must place its foot in the correct location with respect to the obstacle. The correct foot placcment in the top photograph was a matter of good luck. In the middle photograph the foot was too close to the stack of blocks when the leap began, and the foot did not clear. The bottom photograph depicts a leap in which the takcoff point was too far from the obstacie. In all photographs action is from right to left, with lines indicating paths of foot and hip. 
believe that the height and stiffness methods are the most promising for the task of adjusting foot placement for leaping.

\subsection{Behavior During Stance}

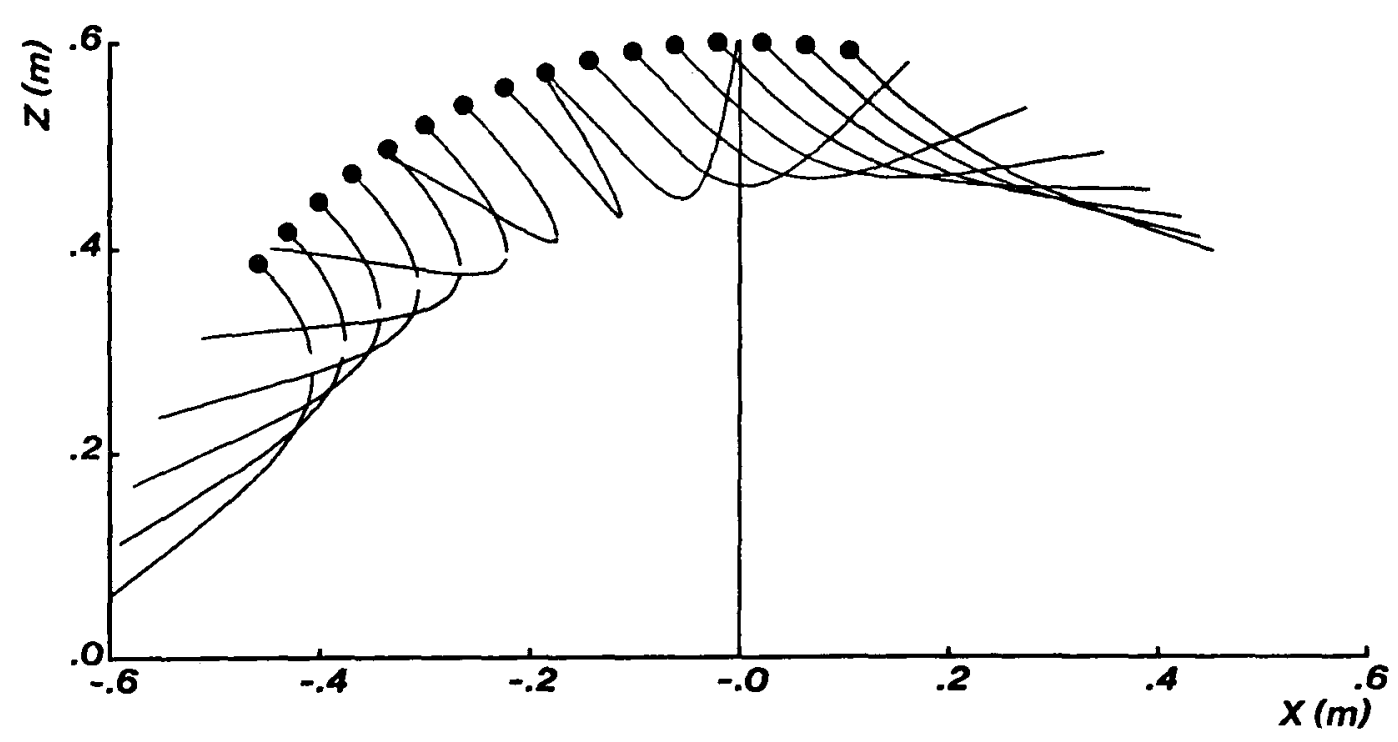

Figure 7-13: Path of the body during stance for different touchdown angles, $\theta_{1}$, ranging from -10 to 50 degrees. Simulation parameters: vertical velocity, $\dot{z}=1 \mathrm{~m} / \mathrm{sec}$; forward velocity, $\dot{x}=1 \mathrm{~m} / \mathrm{sec}$; body mass, $m=16.3 \mathrm{~kg}$; spring constant, $\mathrm{k}=1000 \mathrm{~N} / \mathrm{m}$

Figure 7-13 shows paths of the hopper's body during stance for various touchdown angles. The task of predicting these paths and their corresponding lift-off velocities is difficult; instead, we restrict our attention to the paths which are symmetrical about the vertical axis. These paths result from correct placement of the foot in the center of the CG-print.

The current algorithm to control horizontal velocity hinges on the ability to locate the center of the CG print. The length of the print is estimated by multiplying the forward velocity by the duration of stance. The duration of stance is obtained by measuring the previous stance phase, under the assumption that it does not change appreciably from step to step. In order to maintain the forward velocity of the system, the foot is placed in the center of the CG-print at touchdown.

This mathod of estimating the length of the CG-print is not entirely correct, since the forward velocity of the system does not remain constant throughout stance. Since the foot is placed in front of the body at touchdown, the horizontal component of the force acting on the body by the leg spring serves to decelerate the body. Once the center of gravity of the body has passed over the foot, this force serves to accelerate the 
body. Consequently, the average forward velocity of the hopper during stance is somewhat less than the touchdown velocity. This means that the estimate of the CG-print is more than the actual print, causing the leg to be placed too far forward.

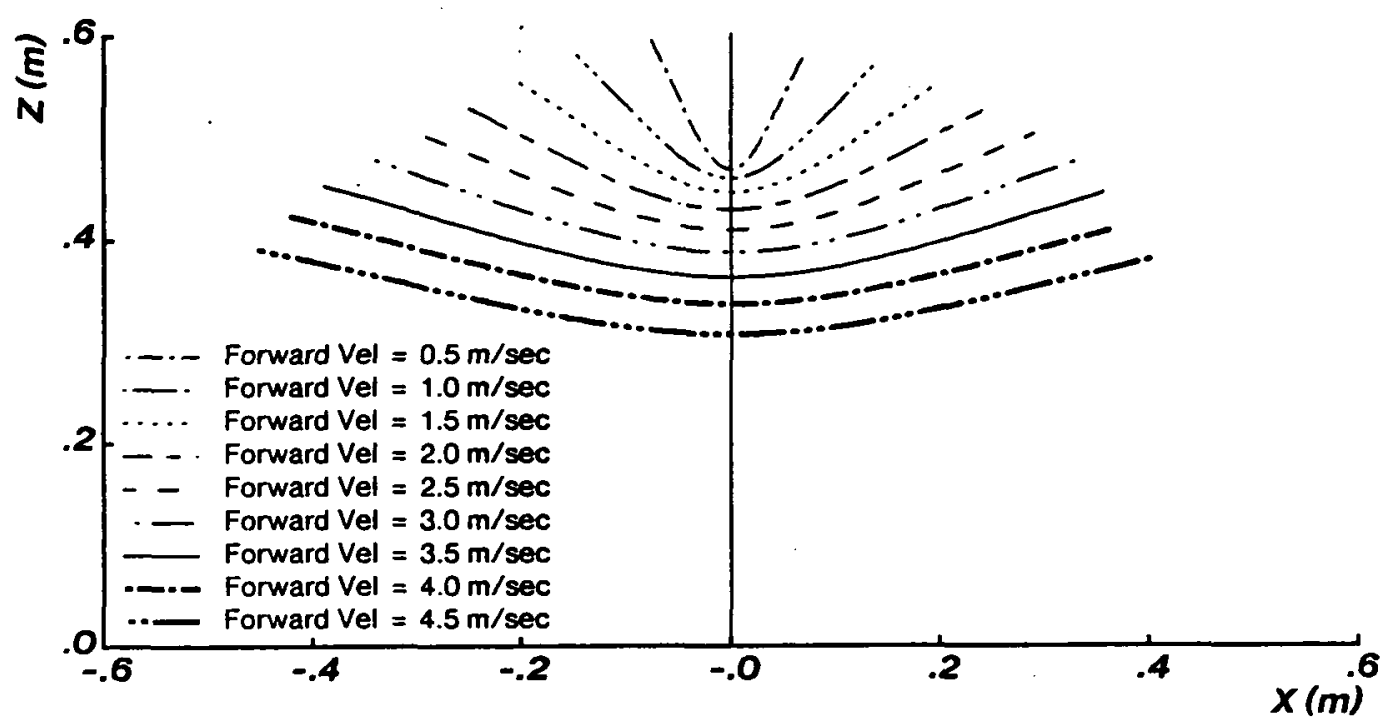

Figure 7-14: Path of the body during stance for different forward velocities. In each case the foot was placed in the center of the CG-print. Simulation parameters: vertical velocity, $z=1.4 \mathrm{~m} / \mathrm{sec}$; body mass, $m=16.3 \mathrm{~kg}$; spring constant, $k=1000 \mathrm{~N} / \mathrm{m}$

The estimate of the length of the CG-print can be improved. An approximate value for the average forward velocity during stance can be computed using simple observations about the path of the body during stance. Figure 7-14 shows simulation data for a planar system. The figure plots the path of the body during stance when the foot is placed in the center of the CG-print for various forward velocities. The body is modeled as a point mass $(16.3 \mathrm{~kg}$ ) affixed to a massless springy leg, with a spring constant of $1000 \mathrm{~N} / \mathrm{m}$. The extended leg exerts a force on the body equal to the body's weight.

Note that each half of the trajectory followed by the body is nearly linear. Thus, the path followed by the hopper's body can be approximated by two line segments. The average decelerating force exerted on the hopper for the first half of the stance phase is approximately equal to the horizontal component exerted on the body while at the middle of the segment. At this point, the leg is retracted to roughly one half of its total retraction. Furthermore, the angle of the leg with respect to the vertical is roughly one half of the touchdown angle (see Figure 7-15).

When the foot is placed in the center of the CG print at touchdown, the leg is maximally retracted halfway through stance, when the leg is vertical. At this point, the vertical component of the body velocity is zero. Therefore, at full retraction the leg must store the kinetic energy of the hopper due to its vertical velocity at 


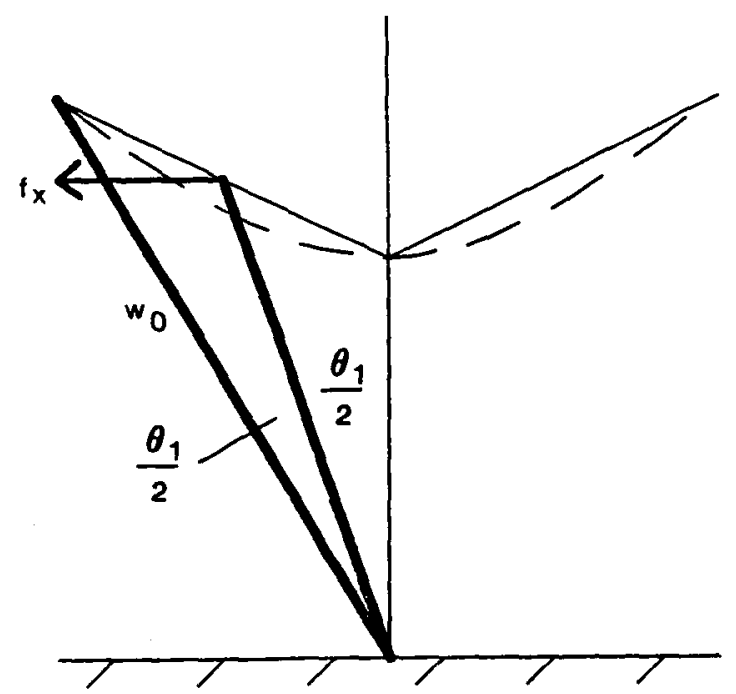

Figure 7-15: Diagram that shows straight-line approximation to the path of the body during stance. Also shown is the point at which the backward force exerted by the leg on the body is approximately equal to the average backward force for the first half of stance.

touchdown and the change in potential energy. Actually, the leg is retracted even further to store some of the kinetic energy due to forward velocity at touchdown. This additional retraction is difficult to calculate; therefore, its effect is ignored.

By setting the kinetic energy due to the vertical velocity at touchdown equal to the potential energy of the leg spring, we have:

$$
\begin{array}{ll}
\frac{\mathrm{m} \dot{\mathrm{z}}^{2}}{2}=\frac{\mathrm{k}\left(\mathrm{w}_{0}-\mathrm{w}\right)^{2}}{2} \\
\mathrm{w}_{-}=\mathrm{w}_{0}-\dot{\mathrm{z}} \sqrt{\frac{\mathrm{m}}{\mathrm{k}}} \\
\begin{array}{ll}
\mathrm{where}, \mathrm{z} & \text { are the vertical position and velocity of the body, } \\
\mathrm{w} & \text { is the length of the leg, } \\
\mathrm{w}_{0} & \text { is the rest length of the leg, } \\
\mathrm{m} & \text { is the mass of the body, and } \\
\mathrm{k} & \text { is the leg spring constant }
\end{array}
\end{array}
$$

The point of half-retraction is given by:

$$
z=w_{0}-\frac{\dot{z}}{2} \sqrt{\frac{m}{k}}
$$


The force exerted by the leg spring is:

$$
f=k\left(w_{0}-z\right)
$$

If $\theta$ is the leg angle at touchdown, then $\theta / 2$ is approximately the angle that locates the body in the center of the line segment. The average force exerted along the horizontal during the first half of the stance phase is:

$$
\mathrm{f}_{\mathrm{x}}=\mathrm{k}\left(\mathrm{w}_{0}-\mathrm{z}\right) \sin \left(\frac{\theta}{2}\right)
$$

The distance the body moves during the first half of stance is:

$$
\Delta x=-\frac{f_{x} T_{S I}^{2}}{8 m}+\frac{\dot{x} T_{s T}}{2}
$$

Therefore, the foot must be placed at the angle specified below:

$$
\Delta x=w_{0} \sin (\theta)
$$

Assuming that $\sin (2 \theta)=2 \sin (\theta)$ and eliminating all intermediate quantities, we have:

$$
\theta=\operatorname{Arcsin}\left[\frac{\dot{x} \mathrm{~T}_{\mathrm{ST}}}{2 w_{0}+(\sqrt{\mathrm{km} \dot{y}}+2 \mathrm{mg})\left(\frac{\mathrm{T}_{\mathrm{ST}}{ }^{2}}{8 \mathrm{~m}}\right)}\right]
$$

Figure 7-16 shows the leg angles required to maintain forward velocity, plotted as a function of the forward velocity. Three lines are shown. The first line was generated from a simulation, and represents the ideal leg angles. The second line was generated using the new method, Eq. (7.13). The third line was produced using the CG-print method. The data show that the angles computed by the new method are substantially closer to the ideal values than those computed by the old method.

The validity of the new method was checked using data generated with a physical 2D hopping machine (Raibert and Brown, 1984). This machine has a leg length, $\mathrm{w}_{0}$, of $0.5 \mathrm{~m}$, a mass, $\mathrm{m}$, of $8.6 \mathrm{~kg}$, and an effective spring constant, $\mathrm{k}$, of $1887 \mathrm{~N} / \mathrm{m}$. Data for three hops are given in Table 7-1.

Note that the predicted lengths of the CG prints based on the new method do indeed fall between the actual prints and the old estimates. The success of this method hinges on the ability to obtain a good estimate for the force excrted on the body by the leg. In order to comply with the derivations given above, a linear force law was used to calculate the values in the table. Measuring the force and fitting the resultant data to a function would provide a more accurate estimate of the length of the CG print. 


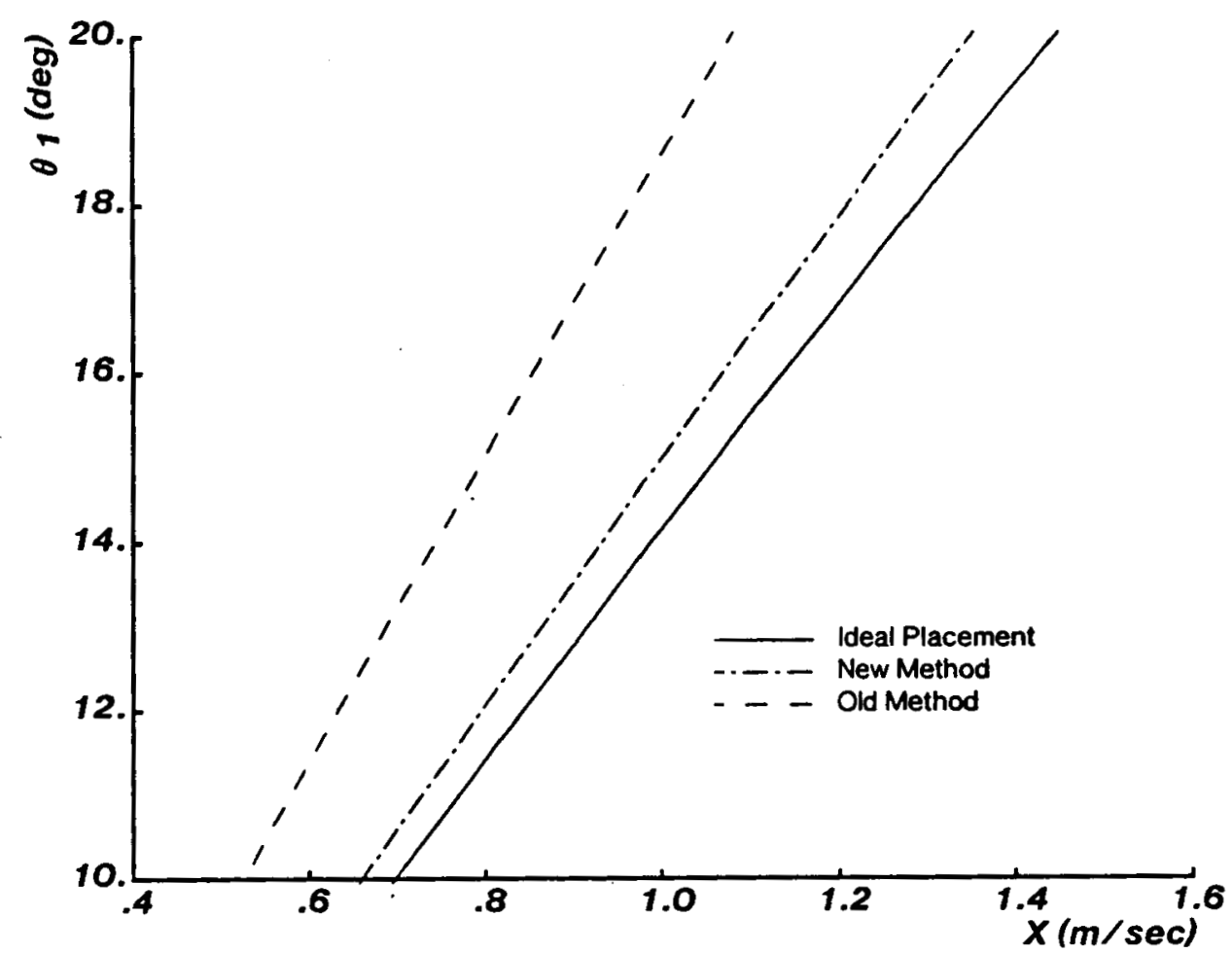

Figure 7-16: Data showing errors with old and new methods for estimating the length of the CG-print Note that the angles computed by the new method are more accurate than those computed by the old method.

$\begin{array}{llll}\text { Parameters } & \underline{\text { Hop } \# 1} & \underline{\text { Hop } \# 2} & \underline{\text { Hop \#3 }} \\ \mathrm{T}_{\mathrm{sT}} & 0.20 \mathrm{sec} & 0.17 \mathrm{sec} & 0.18 \mathrm{sec} \\ \dot{\mathrm{x}} & 0.68 \mathrm{~m} / \mathrm{sec} & 0.28 \mathrm{~m} / \mathrm{sec} & 0.28 \mathrm{~m} / \mathrm{sec} \\ \dot{\mathrm{z}} & 1.30 \mathrm{~m} / \mathrm{sec} & 1.69 \mathrm{~m} / \mathrm{sec} & 1.53 \mathrm{~m} / \mathrm{sec} \\ \text { CG Print (actual) } & 0.119 \mathrm{~m} & 0.042 \mathrm{~m} & 0.032 \mathrm{~m} \\ \text { CG Print (old method) } & 0.136 \mathrm{~m} & 0.048 \mathrm{~m} & 0.050 \mathrm{~m} \\ \text { CG Print (new method) } & 0.129 \mathrm{~m} & 0.045 \mathrm{~m} & 0.048 \mathrm{~m}\end{array}$

Figure 7-1: Data from physical 2D hopping machine that demonstrate accuracy of CG-print estimates. The CG-print estimates generated by the new method more accurately approximate the actual prints than those generated by the old method.

\subsection{Running is Like Juggling}

Claude Shannon visited us this past July, and presented some of his thoughts on juggling:

- Def: The Shannon Common Juggle: The time an object spends in contact with a hand is the same for all objects and all hands. The flight times are equal for all objects thrown from all hands. 
- For a Shannon Common Juggle, the following equation is satisfied:

$$
\frac{N}{H}=\frac{D+F}{D+V}
$$

where:

$\mathrm{N}$ is the number of objects,

$\mathrm{H}$ is the number of hands,

$\mathrm{D}$ is the dwell time, the time each object spends in contact with each hand,

$F$ is the flight time, the time each object spends in the air, and

$\mathrm{V}$ is the vacant time, the time a hand contains no object.

- In his pursuit of devising machines that juggle, Shannon has formulated a theory of juggling that relies on transformations of planar ellipses to represent the motions of each hand. The phase, location, plane, and aspect ratio of the ellipses determine the type and shape of juggle.

These ideas about juggling make me think that juggling and dynamic legged locomotion have much in common:

- Both are cyclic repetitive activities, for which the dynamics of the systems determine the rhythms.

- The terms dwell time, vacant time and flight time for juggling could correspond to stance time, swing time, and flight time for legged locomotion. Note that for a one-legged machine, swing time equals flight time, while for a one ball/one hand juggle, vacant time equals flight time.

- In both juggling and running there are intermittent periods of support. In locomotion each foot touches the ground for a fraction of the leg's step cycle. In juggling, each juggled object touches a hand for only a fraction of the object's trip cycle.

- In both there are bodies that move in a ballistic motion part of the time. Since it is not possible to change the trajectory of a body's motion during the ballistic phase, precise control just before launch is important.

- Shannon has a description of juggling that uses transformations of ellipses that are connected by parabolic arcs. We in locomotion have a theory of balance that decomposes the problem into a planar part and an extra-planar part. If both of these ideas were sound it would mean that 3D juggling and $3 \mathrm{D}$ balance in locomotion might both be understood in terms of planar ideas.

Let us close this section thinking about the game of volleyball, and its relationship to locomotion. Imagine two people warming up for a game of volleyball. The two players stand across the net from each other and hit the ball back and forth. Since they are just warming up, they agree to hit the ball to the each other, rather than to a location where it is hard to get. Each time the ball is in flight, it travels a planar, parabolic path. Each time the ball is handled, its direction is reversed so that it will land in the hands of the other player.

This arrangement of players and ball is very similar to the arrangement of legs and body found in a locomotion system with two legs. The rocking motion of the body that characterizes biped walking and 
running are modeled here by the back and forth motion of the ball. Player contact with the ball is like leg contact with the ground. The primary difference between the two cases is that in volleyball the legs do not travel with the ball, but remain fixed to the ground.

This model also works for a quadruped doing the one-foot. Suppose four players stand at the corners of a rectangle, and hit the ball to each other. Each player hits the ball in a fixed sequence. Each time the ball is in flight, it travels a planar, parabolic path. Each time the ball is handled, the ball is redirected so that it will land in the hands of the next player. The point is that in both the volleyball warmup and in quadruped locomotion, the mass of the system must travel back and forth over the support points in order to sustain the activity.

While what we have described so far corresponds to running in place, it is not hard to image the volleyball players all progressing in the same direction at the same rate while they hit the ball back and forth. In this case the forward motion is superimposed upon the up-and-down bouncing motion, and upon the rocking that permits spacing of the players.

\subsection{Do Locomotion and Manipulation Have a Common ground?}

From time to time I worry about what those working on robot manipulation might learn from results in legged locomotion, and what those of us studying locomotion might learn from the manipulation folks.

My first observation is that in one respect, locomotion is a much harder problem than manipulator control. In particular, the most important state variables in a locomotion system can not be measured directly using simple means. Of course, the internal variables, such as length of a leg, or orientation of the leg with respect to the body, can be measured easily, but the position of the locomotion system in the room and its orientation in space can only be determined using indirect methods. In contrast, there is usually not much trouble determining where the hand of a manipulator is in space, or what its orientation is. One merely starts at the fixed base and proceeds from link to link, using the joint angle sensors and simple kinematic transformations. Furthermore, while the manipulator control system can use a separate motor to directly govern the action of each manipulator joint, the translation and orientation of a locomotion system, at least a dynamic one, can only be controlled indirectly, by making the system bounce, tip, and fall in the desired direction.

My second observation is that in one respect, manipulator control is a much harder problem than locomotion. Our work on locomotion described in this and previous reports, relies on a simple, restricted set of leg and body motions. The hopping motion is just the bouncing that results when springy legs and body are excited in a simple way. We cannot control precisely when the foot will next touch the ground, nor when it will leave the ground. During flight the leg is positioned only with regard to its final end-point, no trajectory control is used. Despite these limitations, we are very happy with the systems' performance.

In contrast, a manipulator control technique that only applied to a restricted subclass of possible motions, or 
one that only worked correctly in a portion of a manipulator's working volume, would be received by other workers in manipulation with nothing short of hostility. Techniques for kinematics, dynamics, trajectory control, and the use of sensors are expected to be general solutions that work everywhere in the work space for the full range of possible joint motions.

My third observation is that one can reconcile these differences between locomotion and manipulation if one recognizes that locomotion is a task, and that manipulator control is a tool. Locomotion is the task of transporting the legged system and its contents from one point to another. Typically, the detailed leg motions required to accomplish the locomotion task are only of indirect interest, perhaps as they affect the time or efficiency of transportation. The motions of the legs are used to accomplish the locomotion goal. Likewise, the task of manipulation is to cause parts to be stacked, placed, inserted, assembled, painted, etc. We shouldn't care what motions the manipulator makes, provided that they accomplish the task.

When one thinks of manipulation in this way, in terms of the task the manipulator and its motions are to accomplish, then the problems of indirect sensing and control are precisely the same for manipulation and locomotion. The manipulation system has the same problem determining the relative positioning of hand and workpiece, as the locomotion system has in figuring out where the payload is with respect to the destination. The manipulation system has the same problem controlling the motions of the parts as the locomotion system has controlling motions of the body.

In the same vein, once one thinks of manipulation in terms of the task to be accomplished, then one is free to use specialized control methods that produce stereotyped motions that are only subsets of the motions the manipulator can make.

Juggling is an example of such a task. Juggling is a form manipulation for which specialized motions have been chosen by the human. The juggler seems to use planar elliptical motions of each hand for a wide range of juggling operations, and the hand motions are very regular, indeed. 


\section{Bibliography}

Agarwal,G.C., Berman,B.M.. Stark,L., Lohnberg,P., Gottleib.G.L. "Studies in postural control systems: Parts I, II, and III." IEEE t. on Systems Science and Cybernetics ssc-6, 2 (1970), 116-132.

Aleshinsky, Zatsiorsky. "Human locomotion in space analyzed biomechanically through a multi-link chain model." J. Biomechanics II (1978), 101-108.

Alexander,R. McN. "The mechanics of jumping by a dog." J. Zoology, Lond. 173(1974), 549-573.

Alexander, R.McN., Langman, V.A.,Jayes, A.S. "Fast locomotion of some African ungulates." Journal of Zoology, London, 183 (1977), 291-300.

Alexander,R. McN. Mechanics of bipedal locomotion. In Perspectives in Experimental Biology, SpencerDavies, P., Ed.,Pergamon, Oxford, , pp. 493-504.

Alexander, R. McN., Goldspink,G.. Mechanics and energetics of animal locomotion. Chapman and Hall, London, 1977.

Alexander, R. McN., Jayes, A.S. "Vertical movements in walking and running." J. Zool., Lond 185 (1978), $27-40$.

Alexander,R. McN., Jayes, A.S. "Fourier analysis of forces exerted in walking \& running." J. Biomechanics 13 (1980), 383-390.

Alexander,R. McN., Vernon,A. "The mechanics of hopping by kangaroos (Macropodidas)." J. Zool., Lond. 177 (1975), 265-303.

Alexander, R.Mc., Maloiy, G.M.O., Ker, R.F., Jayes, A.S., Warui, C.N. "The role of tendon elasticity in the locomotion of the camel (Camelus dromedarius)." J. Zool., London 198 (1982), 293-313.

Anderson,F.. Registration of the pressure power (the force) of the body on the floor during movements, especially vertical jumps., 1st Int. Seminar, Surich, 1967.

Andriacchi, Ogle, Galante. "Walking speed as a basis for normal \& abnormal gait measurements." $J$. Biomechanics 10 (1977), 195-200.

Arsavskii, Y.I., Kots, Y.M., Orlovskii, G.N., Rodionov, I.M., Shik, M.L. "Biophysics of complex systems and mathematical models--Investigation of the biomechanics of running by the dog." Biofizika 10, 4 (1965), 665-672.

Arutyunyan,G.A., Gurfinkel,V.S., Mirskii,M.L. "Organization of movements on execution by man of an exact postural task." Biofizika 14, 6 (1969), 1103-1107.

Asatryan, D.G., Feldman, A.G. "Functional tuning of nervous system with control of movement or maintenance of a steady posture..." Biofizika 10, 5 (1965), 837-846.

Badler, N.I., Smoliar, S.W. The representation of human movements using a digital computer. Tech. Rept. Movement Proj. Report No.9, MS-CIS-78-4, 1977. 
Bair,I.R.. Patent No. 2918738. Volume : Amphibious walking vehicle. , 1959.

Barclay, O.R. "Some aspects of the mechanics of mammalian locomotion." J. of Experimental Biology 30 (1953), 116-120.

Bassmajian,J.V., Tuttle,R. Engineering of locomotion in gorilla and man. In Control of Posture and Locomotion, R.B. Stein, K.G. Pearson, R.S. Smith, J.B. Redford, Eds., Plenum Press, New York, N.Y., 1973, pp. 500-609.

Bavarian, B, Wyman, B.F, Hemami, H. "Control of the constrained planar simple inverted pendulum." Int. J. Control 37, 4 (1983), 741-753.

Beckett,R., Chang,K. "An evaluation of the kinematics of gait by minimum energy." 6 Biomech 1 (1968), 147-159.

Bekker,M.G.. The evolution of locomotion: A conjecture into the future of vehicles, 1961.

Bekker,M.G. "Is the wheel the last word in land locomotion?" New Scientist 17, 248 (1961), 406-410.

Bekker,M.G.. Theory of land locomotion University of Michigan Press, 1962.

Bekker,M.G.. Introduction to terrain-vehicle systems. University of Michigan Press, Ann Arbor, 1969.

Beletskii,V.V. "Biped locomotion dynamics I." Izv. AN SSSR. Mekhanika Tverdogo Tela 10, 3 (1975), 3-14.

Beletskii,V.V. "Dynamics of two-legged walking, II." Izv. AN SSSR. Mekhanika Tverdogo Tela 10, 4 (1975), 3-13.

Beletskii,V.V. "Walking Control and Dynamics of a System with Two Legs." 0,1-11.

Beletskii,V.V., Chudinov,P.S. "Parametric optimization in the problem of biped locomotion." Izv. AN SSSR. Mekhanika Tverdogo Tela 12, 1 (1977), 25-35.

Beletskii, V.V., Chudinov, P.S. "Control of motion of a bipedal walking robot." Izv. AN SSSR. Mekhanika Tverdoga Tela 15,3 (1980), 30-38. UDC 531.8

Beletskii,V.V., Kirsanova,T.S. "Plane linear models of biped locomotion." Izv. AN SSSR. Mekhanika Tverdogo Tela 11, 4 (1976), 51-62.

Bennet-Clark, H.C. "The energetics of the jump of the locust schistocerca gregaria." J.Exp.Biol. 63(1975), 53-83.

Bessonov,A.P., Umnov,N.V.. The analysis of gaits in six-legged vehicles according to their static stability. Proc. Symposium on Theory and Practice of Robots and Manipulators, Udine, Italy, 1973.

Bishop, J.E. Why Do Kangaroos Say to Their Friends, 'Let's Go to the Hop'? 1980 Wall Street Journal.

Bramble, D.M., Carrier, D.R. "Running and breathing in mammals." Science 219 (1983), 251-256.

Bresler, B., Frankel, J.P. "The forces and moments in the leg during level walking." $L$ Amer. Soc. Mech Engs. 27 (1950). 
Brown,L.S.. The right way to walk four-legged. .

Buckett,J. Design of an on-board electronic joint control system for a six-legged vehicle. Ph.D. Th., The Ohio State University, Columbus, Ohio, 1977.

Burnett,C.N., Johnson,E.W. "Development of gait in childhood, Parts I and II." Developmental Medicine in Child Neurology 13, 2 (1971), 196-215.

Burns,M.D.. The control of walking in Orthoptera, I --leg movements in normal walking. J. Exp. Biol., 1973.

Camana,P.C. A study of physiologically motivated mathematical models for human postural control. Ph.D. Th., The Ohio State University, Columbus, Ohio, 1977.

Camana,P.C., Hemami,H., Stockwell,C.W. "Determination of feedback for human posture control without physical intervention." J. of Cybernetics 7 (1977).

Cannon,R.H.,Jr. "Some basic response relations for reaction-wheel attitude control." ARS Journal (1962), 61-74.

Cappozzo, Maini, Marchetti, Pedotti. Analysis of hybrid computer of ground reaction in walking. Proc. IV Int. Seminar on Biomechanics, Philadelphia, Pa., 1973.

Cappozzo, Aurelio, et al. "A General computing method for the analysis of human locomotion." $J$. Biomechanics 8 (1975), 307-320.

Cappozzo, et al. "Interplay of muscular \& external forces in human ambulation." J. Biomechanics 9 (1976), 35-43.

Cavagna, G.A. "Elastic bounce of the body." J. Applied Physiology 29 (1970), 279-282.

Cavagna, G.A. "Force platforms as ergometers." J. Applied Physiology 39 (1975), 174-179.

Cavagna, G.A., Margaria, R. "Mechanics of walking." J. Applied Physiology 21 (1966), 27l-278.

Cavagna, G.A., Saibene, F.P., Margaria, R. "Mechanical work in running." J. of Applied Physiology 19, 2 (1964), 249-256.

Cavagna, G.A., Komarek, L., Mazzoleni, S. "The mechanics of sprint running." J. Physiology 217 (1971), 709-721.

Cavagna, G.A., Thys, H., Zamboni, A. "The sources of external work in level walking and running." J. Physiology 262 (1976), 639-657.

Cavagna, G.A., Heglund, N.C., Taylor, C.R. "Mechanical work in terrestrial locomotion: two basic mechanisms for minimizing energy expenditure." American J. Physiology 233, 5 (1977), R243-R261.

Cavanagh,P.R. "." J. Biomechanics 13 (1979), 397-406.

Ceranowicz, A.Z. Decoupling and finite state control of a five link planar biped model standing on one leg. Master Th., The Ohio State University, Columbus, Ohio, 1977. 
Ceranowicz,A.Z. Planar biped dynamics and control. Ph.D. Th., Department of Electrical Engineering, The Ohio State University, Columbus, Ohio, 1979.

Ceranowicz,A.Z., Syman,B.F., Hemami,H. "Control of constrained systems of controllability index two." IEEE t. on Automatic Control AC-25 (1980).

Cheng,I.S. Computer television analysis of biped locomotion. Ph.D. Th., The Ohio State University, Columbus, Ohio, 1974.

Chow,C.K., Jacobson,D.H. "Studies of human locomotion via optimal programming." Mathematical Biosciences 10 (1971), 239-306.

Chow,C.K., Jacobson,D.H. "Further studies of Human Locomotion: Postural stability and Control." Mathematical Biosciences 15 (1972), 93-108.

Cook,T., Cozzens,B. The initiation of gait. In Neural control of locomotion, R.N. Herman, S.Grillner, P.S. Stein, D.G. Stuart, Eds., Plenum Press, New York, 1976.

Corliss, W.R., Johnson,E.G.. Teleoperator controls. NASA SP-5070, 1968.

Corson, P.E.. Walking tractor. , 1958.

Cotes, J.E., Meade, F. "The energy expenditure and mechanical encrgy demand in walking." Ergonomics (1960).

Cox,W.. Big muskie. New in Engineering, The Ohio State University, Columbus, Ohio, 1970.

Cruse,H.. A new model describing the coordination pattern of the legs of a walking stick insect. Biol. Cybernetics, 1979.

Dawson, T.J. "Energetic cost of locomotion in Australian hopping mice." Nature 259(Jan 1976), 305-307.

Dawson,T.J. "Kangaroos." Scientific American 237, 2 (1977), 78-89.

Dawson, T.J., Taylor, C.R. "Energetic cost of locomotion in kangaroos." Nature 246 (1973), 313-314.

Dougan,S. "The angle of gait." American J. Physiological Anthropology 7 (1924), 275-279.

Drillis,R., Contini,R. Body Segment Parameters. Tech. Rept. Technical Report No. 1166.03, New York University, New York, 1966.

Duysens, J., Loeb, G.E. "Modulation of ipsi- and contralateral reflex responses in unrestrained walking cats." J. of Neurophysiology 44, 5 (1980), 1024-1037.

Ehrlich,A.. Patent No. 1691233. Volume : Vehicle propelled by Steppers, 1928.

Ekerot, C.F., Larson, B., Oscarsson, O. Information carried by the spinocerebellar paths. In Reflex Control of Posture and Movement, Granit, R., Pompeiano, O., Eds., Elsevier/North-Holland Biomedical, New York, 1979, pp. 79-90.

Elftman,H. "A cinematic study of the distribution of pressure in the human foot." Anat. Rec. 59(1934), 481-490. 
Elftman, H. "Forces and energy changes in the leg during walking." Amer. J. Physiol. 125 (1939), 339-356.

Elftman,H. "The basic pattern of human locomotion." Ann. New York Acad. of Sci. Rec. 51 (1951), 1207-1212.

Elfuman,H. "Basic function of the lower limbs." Biomedical Engineering (1967), 342-345.

Engin,A.E. Experimental determination of the patello femoral joint forces. , 29th ACEMB, Boston, Mass., 1976.

Ewald,B.A., Lucas,D.B., Ralston,H.J.. Effect of immobilization of the hip on energy expenditure during level walking. , UCSF Berkeley, 1961.

Farnsworth,R. Gait stability and control of a five link model of biped locomotion. Master Th., The Ohio State University, Columbus, Ohio, 1975.

Fedak, M.A., Pinshow, B., Schmidt-Nielsen, K. "Energy cost of bipedal running." Amer. J. of Physiol. 227, 5 (1977), 1038-1044.

Fenn, W.O. "Work against gravity and work due to velocity changes in running." Amer. J. Physiol. 93 (1930).

Fitch,J.M., Templer,J., Corcoran,P. "The dimensions of stairs." Scientific American 231, 42 (1974).

Foley, et al. "Kinematics of normal child locomotion." J. Biomechanics 12 (1979), 1-6.

Fomin,S.V., Shtilkind,T.I. "The concept of equilibrium of systems having legs." Biofizika 17, 1 (1972), 131-134.

Fomin,S.V., Gurfinkel, V.S., Feldman, A.G., Shtilkind,T.I. "Movements of the joints of human legs during walking." Biophysics 21 (1976), 572-577.

Frank,A.A. Automatic control systems for legged locomotion machines. Ph.D. Th., Univ. of Southern California, Los Angeles, California, 1968.

Frank,A.A. "An approach to the dynamic analysis and synthesis of biped locomotion machines." Medical \& Biological Engineering 8(1970), 465-476.

Frank,A.A. "On the stability of an algorithmic biped locomotion machine." J. of Terramechanics 8, 1 (1971), 41-50.

Frank,A.A. "A method of coordinating the legs of the General Electric walking truck." , ECE-73-3 (1973).

Frank,A.A., McGhee,R.B. "Some considerations relating to the design of autopilots for legged vehicles." Journal of Terramechanics 6, 1 (1969), 23-25.

Frank,A.A., Vukobratovic,M. On the synthesis of biped locomotion machines. In 8th International Conference on Medical and Biological Engineering, , Evanston, Illinois, 1969.

Franklin, R., Bell, W.J., Jander, R. "Rotational locomotion by the cockroach blattella germanica." J. Insect Physiol. 27, 4 (1981), 249-255. 
Friedman, M.B. "Visual control of head movements during avian locomotion." Nature 255, 5503 (1975), 67-69.

Gage,H. "Accelerographic analysis of human gait." ASME Winter Annual Meeting WA $W U, 64$ (1964), 137-152.

Galiana,H.L., Milsum,J.H.. A mathematical model for the walking human leg. 2l'st ACEMB, Houston, Texas, 1968.

Gannett,W.R.. The peripod. , U.S. Army Weapons Command, Rock Island, Illinois, 1967.

Garg,D.P. "Vertical mode human body vibration transmissibility." IEEE t. Systems SMC-6, 2 (1976).

Giralt,G.. Multi-level planning \& navigation system for a mobile robot. , August 1979.

Goddard,R.E., Jr., Hemami,H., Weimer,F.C. "Biped Side Step in the Frontal Plane." ASME Winter Annual Meeting (1981), 147-155.

Goddard Jr. R.E., Hemami, H., Weimer, F.C. "Biped side step in the frontal plane." IEEE Transactions on Automatic Control AC-28, 2 (1983), 179-187.

Golliday,C.L.,Jr. Toward development of biped locomotion control: planar motion of the kneeless biped standing and walking gaits. Ph.D. Th., The Ohio State University, Columbus, Ohio, 1975.

Golliday,C.L.,Jr., Hemami,H. "Postural stability of the two degree-of-freedom biped by general linear feedback." IEEE t. on Automatic Control AC-21, 1 (1976), 74-79.

Golliday,C.L.,Jr., Hemami,H. "An approach to analyzing biped locomotion dynamics and designing robot locomotion controls." IEEE L Automatic Control AC-22, 6 (1977), 963-972.

Graham,D. "Effects of circum-oesophageal lesion on the behavior of the stick insect Carausius morosus, I. Cyclic behaviour patterns." Biol. Cybernetics 32 (1979), 139-145.

Graham,D. "Effects of circum-oesophageal lesion on the behavior of the stick insect Carausius morosus; II. Changes in walking co-ordination." Biol. Cybernetics 32 (1979), 147-152.

Greene, P.H. Problems of organization of motor systems. Quarterly report 29, U. of Chicago Inst. for Comp. Research, 1971.

Greene, P.R., McMahon, T.A. "Running in circles." The Physiologist 22, 6 (1979), S-35 \& S-36.

Greene,P.R., McMahon, T.A. "Reflex stiffness of man's anti-gravity muscles during kneebends while carrying extra weights." J. Biomechanics 12 (1979), 881-891.

Grillner,S. "The role of muscle stiffness in meeting the changing postural and locomotor requirements of force development by the ankle extensors." Acta physiol. scand. 86 (1972), 92-108.

Grillner,S. "Locomotion in vertebrates: central mechanisms and reflex interaction." Physiol. Rev. 55 (1975), 247-304.

Grillner,S. Some aspects on the descending control of the spinal circuits generating locomotor movements. In Neural control of locomotion, Plenum Press, New York, 1976. 
Grillner.S., Zangger, P. "On the central generation of locomotion in the low spinal cat." Experimental Brain Research 34 (1979), 241-261.

Grimes.D.L. An active multi-mode above knee prosthetic controller. Ph.D. Th., Massachusetts Institute of Technology, 1979.

Grundman,J.. Seireg,A. "Computer control of a multitask exoskeleton for paraplegics." On Theory and Practice of Robots and Manipulators -- Second CISM-IFToMM Symposium, Warsaw (1976), 241-248.

Gubina,F.. Stability and dynamic control of certain types of biped locomotion. IV Symp. on External Control of Human Extremities, Dubrov, 1972.

Gubina,F., Hemami,H., McGhee,R.B. "On the dynamic stability of biped locomotion." IEEE $t$ on Biomedical Engineering BME-21, 2 (1974), 102-108.

Gupta.S. Estimation of lower limb joint forces and moments using on-line measurements and computations. Master Th., Ohio State University, Columbus, Ohio, 1975.

Gurfinkel,E.V.. Physical foundation of the stabilography. Symposium International de Posturographic Smolenile, 1973.

Gurfinkel, V.S., Shik, M.L. The control of posture and locomotion. In Motor Control, Gydikou, A.A., Tankou, N.T., Kosarov, D.S., Ed.,Plenum, New York, 1973, pp. 217-234.

Harris,R.I., Beath,T. "Hypermobile flat-foot with short tendo achillis." J. Bone and Joint Surg. 30-a (1948), $116-140$.

Hartrum, T.C. Biped locomotion models. Tech. Rept. Technical Report 272, The Ohio State University, Columbus, Ohio, 1971.

Hartrum,T.C. Computer implementation of a parametric model for biped locomotion kinematics. Ph.D. Th., The Ohio State University, Columbus, Ohio, 1973.

Hatze,H. "A complete set of control equations for the human musculo-skeletal system." J. Biomechanics 10 (1977), 799-805.

Havill,J.R., Ratcliff,J.W. A twin-gyro attitude control system for space vehicles. NASA Technical Note TN D-2419, 1964.

Heglund, N.C.. Taylor, C.R., McMahon, T.A. "Scaling stride frequency and gait to animal size: Mice to horses." Science 186 (1974), 1112-1113.

Hemami,H.. Pole assignment by mode feedback. Proc. 9th Annual Allerton Conference on Circuit and System Theory, Evanston, Ill., 1971.

Hemami,H.. Reduced order models for biped locomotion. Proc. of 7th Pittsburgh Conf. on Modelling and Simulation, Pittsburgh, Pa., 1976.

Hemami,H. "A feedback on-off model of biped dynamics." IEEE $L$ on Systems, Man, and Cybernetics SMC-10, 7 (1980), 376-383. 
Hemami,H., Camana,P.C. "Nonlinear feedback in simple locomotion systems." IEEE t. on Automatic Control 21, 6 (1976).

Hemami,H., Cvetkovic,V.S.. Postural stability of two biped models via Lyapunov second method. Proc. of 1976 JACC, West Lafayette, Indiana, 1976.

Hemami,H., Farnsworth,R.L. "Postural and gait stability of a planar five link biped by simulation." IEEE t. on Automatic Control AC-22, 3 (1977), 452-458.

Hemami,H., Golliday,C.L.,Jr. "The inverted pendulum and biped stability." Mathematical Biosciences 34 (1977), 95-110.

Hemami,H., Jaswa,V.C. "On a three-link model of the dynamics of standing up and sitting down." IEEE t. Systems, Man, and Cybernetics SMC-8, 2 (1978), 115-120.

Hemami, H., Katbab, A. "Constrained inverted pendulum model for evaluating upright postural stability." J. of Dynamic Systems, Measurement, and Control 104 (1982), 343-349.

Hemami,H., Weimer,F.C. Further considerations of the inverted pendulum. , Proc. of the Fourth Iranian Conference on Electrical Engineering, Pahlavi University, Shiraz, Iran, 1974, pp. 697-708.

Hemami, H., Weimer, F.C. "Modeling of nonholonomic dynamic systems with applications." J. of Applied Mechanics, Paper \#81 - APM - 12 ASME (1981), 1-6.

Hemami,H., Wyman,B.F. "Modeling and control of constrained dynamic systems with application to biped locomotion in the frontal plane." IEEE L on Automatic Control AC-24, 4 (1979), 526-535.

Hemami,H., Wyman,B.F. "Indirect control of the forces of constraint in dynamic systems." Journal of Dynamic Systems, Measurement, and Control 101 (1979), 355-360.

Hemami, H., Yuan-Fang, Z. Initiation of walk and tiptoe of a planar nine-link biped. In , , 1982, Chap. Mathematical Biosciences, pp. 163-189.

Hemami, H., Zheng, Y. "Initiation of walk and tiptoe of a planar nine-link biped." Mathematical Biosciences $61,(1982), 163-189$.

Hemami,H., Weimer,F.C., Koozekanani,S.H. "Some aspects of the inverted pendulum problem for modelling of locomotion systems." IEEE $L$ on Automatic Control AC-18, 6 (1973), 658-661.

Hemami,H., Weimer,F.C., Robinson,C.S., Stockwell,C.W., Cvetkovic,V.S. "Biped Stability Considerations with Vestibular Models." IEEE Tran. on Automatic Control AC-23, 6 (1978), 1074-1079.

Hemami,H., Tomovic,R., Ceranowicz,A.Z. "Finite state control of planar bipeds with application to walking and sitting." J. Bioengineering 2 (1979), 477-494.

Hemami,H., Wall,C.,III, Black,F.O. "Single inverted pendulum biped experiments." J. of Interdisciplinary Modeling and Simulation 2(3) (1979), 211-227.

Hemami,H., Robinson,D.J., Ceranowicz,A.Z. "Stability of planar biped models by simultaneous pole assignment and dccoupling." Int. J. Sysiems Science 11, 1 (1980), 65-75. 
Hemami, H., Hines, M.J., Goddard. R.E., Friedman, B. "Biped sway in the frontal plane with locked knees." IEEE Transactions on Systems, Man, and Cybernetics SMC-12, 4 (1982), 577-582.

Hemami,H., Robinson,D.J., Ceranowicz,A.Z. "On stability, pole assignment and decoupling of some biped modeis." Submitted to IEEE $L$ on Automatic Control 0.

Hemami,H., Weimer,F.C., Robinson,C.S., Stockwell,C.W., Cvetkovic,V.S. "Analysis of some derived models of otoliths and semicircular canals." 1977 JACC 0.

Herman,B., Cook,T., Cozzens,B., Freeman,W. Control of postural reaction in man: the initiation of gait. In Control of Posture and Locomotion, R.B. Stein, K.G. Pearson, R.S. Smith, J.B. Redford, Eds., Plenum Press, New York, N.Y., 1973, pp. 353-388.

Hicks,J.H. The three weight bearing mechanisms of the foot. In in: Biomechanical Studies of the MusculoSkeleton System, F.G. Evans, C.C. Thomas, Eds., , Springfield, Ill., 1961.

Higdon.D.T., Cannon,R.H., Jr. On the control of unstable multiple-output mechanical systems. Proc. of Winter Annual Meeting, ASME Winter Annual Meeting, 1963.

Hildebrand, "Motion of the running cheetah and horse." J. Mammalogy 40, 4 (1959), 481-495.

Hildebrand,M. "How animals run." Scientific American (1960), 148-157.

Hildebrand, "Symmetrical gaits of horses." Science 150 (1965), 701-708.

Hildebrand,M. "Analysis of the symmetrical Gaits of Tetrapods." Folia Biotheoretica 4(1966), 9-22.

Hill,J.C. A model of the human postural control system. , 8th IEEE Symp. Adaptive Processes: Decision and Control, 1969.

Hirose,S., Umetani,Y.. The basic motion regulation system for a quadruped walking vehicle. ASME Conference on Mechanisms, Los Angeles, 1980.

Hoffer, J.A., O’Donovan, M.J., Pratt, C.A., Loeb, G.E. "Discharge patterns of hindlimb motoneurons during normal cat locomotion." Science 213 (1981), 466-468.

Horn, B.K.P. The fundamental EEL equations. Working Paper 116, MIT, AI:, 1975.

Howell,R.. Speed in animals. Univ. of Chicago Press, Chicago, 1944.

Hristic,D., Vukobratovic,M.. A new approach to the rehabilitation of paraplegic persons. , 1971.

Hughes,G.M. "The coordination of insect movements." J. Exp. Biol. 29 (1952), 267-285.

Jain,A.K. Measurement of gait in children. Tech. Rept. Tech. Note No.1, The Ohio State University, 1972. Communication and Control Systems Lab, Dept. of Electrical Engineering

Jain,A.K. A Study of regularly realizable gait matrices. Master Th., The Ohio State University, Columbus, Ohio, .

Jaswa,V.C. Dynamic stability and control of a three link model of a human being. Master Th., Ohio State

University, Columbus, Ohio, 1975. 
Jaswa,V.C. An experimental study of real-time computer control of a hexapod vehicle. Ph.D. Th., Ohio State University, Columbus, Ohio, 1978.

Jayes, A.S., Alexander, R.McN. "The gaits of chelonians: walking techniques for very low spceds." J. Zool. Lond. 191 (1980), 353-378.

Johnston.R.C., Smidt,G.L. "Measurement of hip-joint motion during walking." J. Bone \& Joint Surg. 51-A (1969), 1083-1094.

Jones,R.L. "The human foot -- An experimental study of its mechanics and the role of its muscle and ligaments in the support of the arch." Am. J. Anat. 68 (1941), 1-39.

Jones,F.W.. Structure and function as seen in the foot, Edition 2. Bailliere, Tindall and Cox, London, 1949.

Jones, G.M. "Observations on the Control of Stepping \& Hopping Movements in Man." J. Physiol 219 (1971), 709-727.

Juricic,D., Vukobratovic,M. "Mathematical modelling of bipedal walking system." ASME Publication $72-$ WA BHF-13(1972).

Kamon, E. "Electromyographic kinesiology of jumping." Archives of Physical Medicine \& Rehab. 52 (1971), 152-157.

Kanayama, Yutaka, Iijima, Jun'ichi, Ochiai, Hajime, Watarai, Hiroshi, Ohkaw, Kohichi. A self-contained robot 'Yamabiko'. , 3rd USA-Japan Computer Conference, 1978.

Kane,T.R., Levinson,D.A. "Formulation of equations of motion for complex spacecraft." J. of Guidance and Control 3, 2(1980), 99-112.

Katbab, A. Three-dimensional torso model with muscle actuators. OSU, EE Dept. DI-83-HH-093

Kato,I., Tsuiki,H. The hydraulically powered biped walking machine with a high carying capacity. In IV Symp. on External Control of Human Extremities, , Dubrovnik, Yugoslavia, 1972.

Kato,I., Matsushita,S., Kato,K. A model of human posture control system. In Advances in External Control of Human Extremities, M.M. Gavrilovic, A.B. Wilson Jr., Eds., , Belgrade, 1969, pp. 443-464.

Kato, T., Takanishi, A., Jishikawa, H., Kato, I. The realization of the quasi-dynamic walking by the biped walking machine. 4th Symposium on Theory and Practice of Robots and Manipulators, IFTMoM, 1981.

Keith,A. "The history of the human foot and its bearing on orthopedic practice." J. Bone and Joint Surg. II (1929), 10-32.

Kenny,J.D. Investigation for a walking device for high efficiency lunar locomotion. Proceedings of American Rocket Society Annual Meeting, Philadelphia, 1965.

Kettelkamp,D.B., Johnston,R.C., Smidt,G.L., Chao,E.Y.S., Walker,M. "An electrogoniometric study of knee motion in normal gait." J. Bone \& Joint Surg. 51-A (1970), 775-790.

Khadelwal,B.M., Frank,A.A. On the dynamics of an elastically coupled multi-body biped locomotion model. Procecdings of Joint Automatic Control Conference, Austin, Texas, 1974. 
Khosravi-Sichani, B. A preliminary study of a two segment artificial foot. Master Th., The Ohio State University, Columbus, Ohio, 1979.

Kinch,E.A.. Patent No. 1669906. Volume : Vehicle propelling device. , 1928.

Klein,C.A., Briggs,R.L. "Use of active compliance in the control of legged vehicles." IEEE t. on Systems, Man, and Cybernetics SMC-10, 7 (1980), 393-400.

Klien, C.. Limb motion for a 3 legged walking machine. , 1980.

Kljajic,M., Trnkoczy,A. "A study of adaptive control principle orthoses for lower extremitics." IEEE $L$ On Systems, Mans, and Cybernetics SMC-8, 4 (1978), 313-321.

Koozekanani,S.H., McGhee,R.B. "Occupancy problems with pairwise exclusion constraints -- an aspect of gait enumeration." Journal of Cybernetics 2, 4 (1973), 14-26.

Koozekanani,S.H., Stockwell,C.W., McGhee,R.B., Firoozmand,F. "On the role of dynamic models in quantitative posturography." IEEE L Biomedical Engineering (1980).

Kuee, B. "Movement of two legged system of pendulum type." Mekhanika Tverdogo Tela 2(1975), 58-61. In Russian

Kugushev,E.I., Jaroshevskij, V.S.. Problems of selecting a gait for an integrated locomotion robot. Institute of Applied Mathematics, USSR Academy of Sciences, Moscow, .

Larin,J.B. "Stabilization of biped walking apparatus." Izv. AN SSSR. Mekhanika Tverdogo Tela 11, 5 (1976), 4-13.

Larin, V.B. "Control of a jumping robot, I. Choice of programmed trajectory." 14, 6 (1979), 27-32.

Leimanis,E.. The general problem of the motion of coupled rigid bodies about a fixed point. springer-Verlag, 1965.

Liberson, W.T. "Biomechanics of gait: a method of study." Arch. Phys. Med. Rehabilitation (1965).

Lindholm,L.E., Oberg,K.E.T.. An optoelectronic instrument for remote on-line movement monitoring. Chalmers University of Technology, Goteborg, Sweden, 1979.

Lisin, V.V., Frankstein, S.I., Rechtmann, M.B. "The influence of locomotion on flexor reflex of the hind limb in cat and man." Experimental Neurology 38 (1973), 180-183.

Liston,R.A.. Walking machine. Annual Meeting of the American Society of Agricultural Engineers, University of Georgia, 1965.

Liston,R.A., Mosher,R.S. A versatile walking truck. Proc. Transportation Engineering Conference, ASME, Washington, D.C., 1968.

Loeb, G. E. "Somatosensory unit input to the spinal cord during normal walking." Canadian J. of Physiology and Pharmacology 59, 7 (1981), 627-635.

Lucas, E. "La machine a marcher." Recreations Mathematiques (Huitieme Recreation) 4(1894). In French 
Mackerle.J. "Walking riders." T-68 12 (1968), 776-777.

Manter,J. "Dynamics of quadrupedal walking." J. of Experimental Biology 15, 4 (1938), 522-539.

Margaria, R. Biomechanics and energetics of muscular exercise. In , Oxford Univ. Press, Walton St., Oxford, 1976.

Matsuoka, K. "Study on robot legs." Bulletin of ME Dept. 11 (1976), 65-69. In Japanese

Matsuoka,K. A model of repetitive hopping movements in man. Proc. of Fifth World Congress on Theory of Machines and Mechanisms, IFIP, 1979.

Matsuoka,K. "A mechanical model of repetitive hopping movements." Biomechanisms 5(1980), 251-258. In Japanese

McGhee,R.B.. Finite state control of quadriped locomotion. Proc. of the Int. Symp. on External Control of Human Extremities, Dubrovnik, Yugoslavia, 1967.

McGhee,R.B. "Finite state control of quadruped locomotion." Simulation (1967).

McGhee,R.B. "Some finite state aspects of legged locomotion." Math. Biosciences. 2 (1968), 67-84.

McGhee,R.B.. A mathematical theory for legged locomotion systems. Proc. 1970 Midwest Symposium on Circuit Theory, 1970.

McGhee,R.B. Robot locomotion with active terrain accommodation. , Proc. NSF Robotics Research Workshop, Univ. Rhode Island, 1980.

McGhee, R.B. Vehicular legged locomotion. To appear in Advances in Automation and Robotics

McGhee,R.B., Buckett,J.R. "Hexapod." Interface Age (1977).

McGhee,R.B., Frank,A.A. "On the stability properties of quadruped creeping gaits." Math Biosciences. 3 (1968), 331-351.

McGhee,R.B., Frank,A.A. "Some considerations relating to the design of autopilots for legged vehicles." J. of Terramechanics 6, 1 (1969).

McGhee,R.B., Iswandhi,G.I. "Adaptive locomotion of a multilegged robot over rough terrain." IEEE. $L$ on Systems, Man, and Cybernetics SMC-9, 4 (1979), 176-182.

McGhee,R.B., Jain,A.K. "Some properties of regularly realizable gait matricies." Mathematical Biosciences 13, 1/2 (1972), 179-193.

McGhee,R.B., Kuhner,M.B. On the dynamic stability of legged locomotion systems. Advances in External Control of Human Extremities, Belgrade, 1969, pp. 431-442.

McGhee,R.B., Orin,D.E.. An interactive computer control system for a quadruped robot. Proc. Symposium on Theory and Practice of Robots and Manipulators, Udine, Italy, 1973.

McGhee,R.B., Pai,A.L. "An approach to computer control for legged vehicles." J. Terramechanics II (1972), 9-27. 
McGhee,R.B., Sun,S.S.. On the problem of selecting a gait for a legged vehicle. Proc. of VI IFAC Symposium on Automatic Control in Space, Tsakhkadzor, Armenian SSR, U.S.S.R., 1974.

McGhee,R.B., Koozekanani,S.H., Weimer,F.C., Rahmani,S. Dynamic modelling of human locomotion. Proceedings of Joint Automatic Control Conference, 1979.

McKenney,J.D. Investigation for a walking device for high efficiency lunar locomotion. Tech. Rept. Paper 2016-61, American Rocket Society Space Flight Report to the Nation, New York, 1961.

McMahon, T.A. "Using body size to understand the structural design of animals: quadrupedal locomotion." Journal of Applied Physiology 39", 4 (1975), 619-627.

McMahon, T.A. Allometry. In 1977 Yearbook of Science and Technology, McGraw-Hill, 1976, pp. 49:57.

McMahon, T.A. "Gravitational scale effects." The Physiologist 22, 6 (1979), S-5 \& S-6.

McMahon, T.A. "Scaling Physiological Time." American Mathematical Society 13 (1980), 131-163.

McMahon,T.A., Greene,P.R. "Fast running tracks." Scientific American 239, 6 (1978), 148-163.

McMahon, T.A., Greene, P.R. "The influence of track compliance on running." J. Biomechanics 12 (1979), 893-904.

Melvill-Jones, G. and Watt, D.O.D. "Observations on the control of stepping and hopping movements in man." J. Physiol. 219 (1971a), 709-727.

Melvill-Jones, G. and Watt, D.O.D. "Muscular control of landing from unexpected falls in man." J. Physiol. 219 (1971b), 729-737.

Mihajlov,D., Chang,C.W., Bekey,G.A., Perry,J. "Computer graphics in the study of normal and pathological human gait." Medinfo 77 (1977), 561-564.

Miller,D.I., Nelson,R.C.. Biomechanics of sport. Lea \& Febiger, Philadelphia, Pennsylvania, 1973.

Miller, S., VanderBurg, J., VanderMeche, F.G.A. Locomotion in the cat: basic programmes of movement. In Brain Research, Elsevier Scientific Pub. Co., 1975, pp. 239-253.

Miller, S., VanderBurg, J., VanderMeche, F.G.A. Coordination of movements of the handlimbs and forelimbs in different forms of locomotion in normal and decerebrate cats. In Brain Research, Elsevier Scientific Pub. Co., Amsterdam, 1975, pp. 217-237.

Milner,M. et al. "Angle diagram in the assessment of locomotor function." S.A. Medical Journal 47 (1973), 951-957.

Milner,M., Brennan,P.K., Wilberforce,C.B.A. "Stroboscopic polaroid photography in clinical studies of human locomotion." S.A. Medical Journal 47 (1973), 948-950.

Miura, H., Shimoyama, I. "Computer control of an unstable mechanism." J. Fac. Eng. , 17 (1980), 12-13. In Japanese

Miura, H., Shimoyama, I. Dynamical Walk of Biped Locomotion. First International Symposium of Robotics Research, MIT Press, 1983. 
Miyazaki, Fumio. "A Control Theoretic Study and Dynamic Linked Locomotion." ? (1980).

Mocci,U., Petternella,M., Salinari,S. Experiments with six-legged walking machines with fixed gait. Tech. Rept. Report 2-12, Institute of Automation, University of Rome, 1972.

Mochon, S. "A mathematical model of human walking." Lectures on Math. in the Life Sciences 14 (1981), 193-213.

Mochon, S., McMahon, T.A. "Ballistic Walking: An improved model." Mathematical Biosciences 52 (1980), 241-260.

Morrison,R.A. Iron mule train. , Proc. Off-Road Mobility Research Symposium, Washington, D.C., 1968, pp. 381-400.

Morrison,J.B. "The mechanics of the knee joint in relation to normal walking." J. Biomechanics 3(1970), 51-61.

Morrison,J.B. "The mechanics of muscle function in locomotion." J. Biomechanics 3(1970), 431-461.

Morton,D.J.. The human foot. Columbia University Press, New York, 1935.

Morton,D.J.. Human Locomotion and Body Form. , University of Columbia, Baltimore, 1952.

Mosher,R.S. Design and fabrication of a full-scale, limited motion pedipulator. General Electric report, 1965.

Mosher,R.S. Test and evaluation of a versatile walking truck. Proc. Off-Road Mobility Research Symposium, International Society for Terrain Vehicle Systems, 1968, pp. 359-379.

Murphy, K., Raibert, M.H. Trotting and Bounding in a Planar Two-Legged Model. Conference on Theory and Practice of Robots and Manipulators, IFTMoM, 1984.

Murray,M.P. "Gait as a total pattern of movement." Am. J. Phys. Med. 46, 1 (1967), 290-333.

Murray,M.P., Drought,A.B., Kovry,R.C. "Walking patterns of normal men." J. Bone \& Joint Surg. 46-A, 2 (1964), 335-360.

Murray,M.P., Seirig,A., Scholz,R.C. "Center of gravity, center of pressure and supportive forces during human activities." J. of Applied Pysiology 23, 6 (1967), 831-838.

Murthy, S.S., Raibert, M.H. 3D Balance in Legged Locomotion: Modeling and Simulation for the OneLegged Case. Inter-Disciplinary Workshop on Motion: Representation and Perception, ACM, 1983.

Muybridge,E.. The human figure in motion. Dover Publications, New York, 1955. First published in 1901, Chapman and Hall, Ltd., London.,

Muybridge,E.. Animals in motion. Dover Publications, New York, 1957. First Published in 1899, Chapman and Hall, Ltd., London

Napier,J. "The antiquity of human walking." Scientific American 216, 4 (1967), 56-66. 
Narinyani,A.S., Pyatkin,V.P., Kim,P.A., Dementyev,V.N. "Walking robot: a non-deterministic model of control." 0 .

Narinyani,A.S., Patkin,V.P., Kim,P.A. Walking robot: a non-deterministic model of control. , Siberian Brnch, Novosibirs, .

Nashner, L.M. "A model describing vestibular detection of body sway motion." Acta Otolaryng. 72 (1971), 429-436.

Nashner, L.M. Vestibular and reflex control of normal standing. In Control of Posture and Locomotion, R.B. Stein, K.G. Pearson, R.S. Smith, J.B. Redford, Eds., Plenum Press, New York, N.Y., 1973, pp. 291-308.

Nashner, L.M. "Adapting reflexes controlling the human posture." Experimental Brain Research 26 (1976), 59-72.

Nashner, L.M. "Fixed patterns of rapid postural responses among leg muscles during stance." Experimental Brain Research 30 (1977), 13-24.

Nashner, L.M. "Visual contribution to rapid motor responses during postural control." Brain Research 150 (1978), 403-407.

Nashner, L.M. "Balance adjustments of humans perturbed while walking." J. Neurophysiology 44, 4 (1980), 650-664.

Nashner, L.M.. Woollacott, M., Tuma, G. "Organization of rapid responses to postural and locomotor-like perturbations of standing man." Experimental Brain Research 36 (1979), 463-476.

Nilson,F.A.. Supporting and Propelling Mechanism for Motor Vehicles. 1926.

Okhotsimskii,D.E., Platonov,A.K.. Control algorithm of the walker climbing over obstacles. Proc. UJCAI, Stanford, California, 1973.

Okhotsimskii,D.E., Platonov,A.K., et al.. Control algorithms of legged vehicle capable of mastering obstacles. Proc. of Sth IFAC Symp. on Automatic Control in Space, Geneva, 1973.

Okhotsimskii,D.E., Gurfinkel,V.S., Devyanin,E.A., Platonov,A.K. Integrated walking robot development. Conference on Cybernetic Models of the Human Neuromuscular System, 1977.

O'Leary, D.P., Ravasio, M.J. Simulation of vestibular semicircular canal afferent responses during righting movements of a freely falling cat.

Orin,D.E. A simulation study of a computer-assisted manual control system for legged vehicles. Master Th., The Ohio State University, Columbus, Ohio, 1972.

Orin,D.E. Interactive control of a six-legged vehicle with optimization of both stability and energy. Ph.D. Th., The Ohio State University, Columbus, Ohio, 1976.

Orin,D.E. "A three degree-of-freedom leg for a hexapod locomotion vehicle." (1980).

Orin,D.E. "CARM: General-purpose subroutine package for robot linkage systems simulation." (1980). 
Orin, D.E. Supervisory control of a multilegged robot. Tech. Rept. Tech Note \#23, EE, Ohio State Univ., Nov, 1981.

Orin,D.E., Oh,S.Y. "Determination of joint positions from limb segment constraints in robotic systems." Proc of Fifth World Congress on Theory of Machines and Mechanisms (1979), 860-863.

Orin,D.E., McGhee,R.B., Vukobratovic,M. "Kinematic and kinetic analysis of open-chain linkages utilizing Newton-Euler methods." Mathematical Biosciences 43(1979), 107-130.

Pai,A.L. Stability and control of legged locomotion systems. Ph.D. Th., The Ohio State University, Columbus, Ohio, 1971.

Pearson,K. "The control of walking." Scientific American (1976), 72-86.

Pedley, T.J.. Scale effects in animal locomotion. Academic Press, London, 1977.

Pedotti,A. "A study of motor coordination and neuromuscular activities in human locomotion." Biological Cybernetics 26 (1977), 53-62.

Pedotti,A., et al. "Optimization of muscle-force sequencing in human locomotion." Math Bioscience 28 (1978), 57-76.

Pennycuick, C.J. "On the running of the gnu (Connochaetes Taurinus) and other animals." J. Exp. Biol. 63 (1975), 775-799.

Perry,J. Foot floor contact guide. Rancho Los Amigos Hospital Kinesiology Service.

Petternella,M., Salinari,S. Six legged walking vehicles. Tech. Rept. Report No.74-31, University of Rome, 1974. Istituto di Automatica, Rome Italy

Plagenhoef, S.. Patterns of Human Locomotion: A Cinematographic Analysis. Prentice Hall, Englewood Cliffs, New Jersey, 1971.

Pringle, J.W.S. The reflex mechanism of the insect leg. In , , 1939, pp. 8-17.

Pugh, L.G.C.E. "The influence of wind resistance in running and walking and the mechanical efficiency of work against horizontal or vertical forces." J. Physiology 213(1971), 255-276.

Raibert, M.H. Dynamic stability and resonance in a one legged hopping machine. Conference on Theory and Practice of Robots and Manipulators, IFTMoM, 1981.

Raibert, M.H., Brown, H.B., Jr., Murthy, S.S. 3D Balance using 2D Algorithms? Proceedings of Advanced Study Institute on Robotics and Artificial Intelligence, NATO, 1983.

Raibert, M.H. "Hopping in legged systems -- Modelling and simulation for the 2D one-legged case." IEEE Tran. Systems, Man, and Cybernetics, 3 (1984), In Press.

Raibert, M.H., Brown, H.B.,Jr. "Experiments in balance with a 2D one-legged hopping machine." $A S M E$ J. Dynamic Systems, Measurement, and Control, In Press (1984).

Raibert, M.H., Sutherland, I.E. "Machines That Walk." Scientific American 248, 1 (1983), 44-53. 
Raibert, M.H., Wimberly, F.C. "Tabular control of balance in a dynamic legged system." IEEE Tran. Systems, Man, and Cybernetics, In Press (1984).

Raibert, M.H., Brown, H.B.,Jr., Chepponis, M., Hastings, E., Shreve, S.T., Wimberly, F.C. Dynamically Stable Legged Locomotion. Tech. Rept. CMU-RI-81-9, Robotics Institute, Carnegie-Mellon University, 1981.

Raibert, M.H., Brown, H.B.Jr., Chepponis, M., Hastings, E., Murthy, S.S., Wimberly, F.C. Dynamically Stable Legged Locomotion -- Second Report to DARPA. Tech. Rept. CMU-RI-TR-83-1, Robotics Institute, Carnegie-Mellon University, 1983.

Raibert. M.H., Brown, H.B.Jr., Chepponis, M., Hastings, E., Koechling, J., Murphy, K., Murthy, S.S., Stentz, A. Dynamically Stable Legged Locomotion -- Third Annual Report. Tech. Rept. CMU-RI-TR-83, Robotics Institute, Carnegie-Mellon University, 1983.

Raibert, M.H., Brown, H.B., Jr., Murthy, S.S. 3D Balance using 2D Algorithms? First International Symposium of Robotics Research, MIT Press, 1983.

Raibert, M.H., Brown, H.B.Jr., Chepponis, M. "Experiments in balance with a 3D one-legged hopping machine." International Journal of Robotics Research 3, 2 (1984).

Ralston,H.J. "Energy-spced relation and optimal speed during level walking." Int. Z. Angew. Physical Einsch Arbeits Physiol. Bd. 17 (1958), 277-283.

Ralston,H.J. Energetics of human walking. In In Neural Control of Locomotion, R.N. Herman, S.Grillner, P.S. Stein, D.G. Stuart, Eds., Plenum Press, New York, 1975, pp. 77-98.

Ramey, M.R. "Force relationship of the running long jump." Medicine and Science in Sports 2, 3 (Fall 1970), 146-151.

Roberts,W.M., Levine,W.S., Zajac,F.E.,III. "Propelling a torque controlled baton to a maximum height." IEEE L. Automatic Control AC-24, 5 (1979), 779-782.

Robinson,D.J., Stockwell,C.W., Koozekanani,S.. A method for evaluation of vestibular postural control in humans. Boston, Mass., 1976. 29th ACEMB

Russell,M., Jr. "Odex I: The First Functionoid." Robotics Age 5, 5 (1983), 12-18.

Rygg,L.A.. Mechanical horse, 1893.

Saltzman,E. "Levels of sensorimotor representation." J. Mathematical Psychology 20, 2 (1979).

Saund, E. "The physics of one-legged mobile robots - Part I - A general discussion." Robotics Age (Sep/Oct 1982), 38-45.

Saund, E. "The physics of one-legged mobile robots - Part II - The mathematical description." Robotics Age (Nov/Dec 1982), 35-37, 53.

Saunders,J.B., Imma,V.T., Eberhart,H.D. "The major determinants in normal and pathological gait." The Journal of Bone \& Joint Surg. 35-A, 3 (1953), 543-558. 
Schneider,A. Yu., Gurfinkel,E.V., Kanaev,E.M., Ostapchuk,V.G. A system for controlling the extremities of an artificial walking apparatus. In Russian Report No.5, Physio-Technical Institute, 1974. General and Molecular Physics Series, Moscow, USSR

Schwarts,R.P.. Heath,A.L., Morgan,D.W., Towns,R.C. "A quantitative analysis of recorded variables in the walking patterns of normal adults." J. Bone \& Joint Surg. 46-A (1964), 324-334.

Seifert,H.S. "The lunar pogo stick." J. Spacecraft and Rockets 4, 7 (1967).

Seifert,H.S. Small scale lunar surface personnel transporter employing the hopping mode. Tech. Rept. Report No.397, Stanford University Dept. Aeronautics and Astronautics, 1968, 1970.

Seireg,A., Arvikar,R.J. "A mathematical model for the evaluation of forces in lower extremities of the musculo-skeletal system." J. of Biomechanics 6 (1973), 313-326.

Severin,F.V., Orlovskii,G.N., Shik,M.L. "Work of the muscle receptors during controlled locomotion." Biophysics 12, 3 (1967), 575-586.

Shigley,R. Mechanics of walking vehicles. Tech. Rept. Report No. 7, OTAC, Detroit, Michigan, 1957. Land Locomotion Laboratory

Shik,M.L., Orlovskii,G.N. Coordination of the limbs during running of the dog. Institute of Biological Physics, 1965. Academy of Sciences, Moscow, U.S.S.R.

Shik, M.L., Orlovsky, G.N., Severin, F.V. "Locomotion of the mesencephalic cat elicited by stimulation of the pyramids." Biofizika 13 (1968), 127-135.

Simons, J., Van Brussel, H., De Schutter, J., Verhaert, J. "A self-learning automaton with variable resolution for high precision assembly by industrial robots." IEEE $L$ Automatic Control AC-27, 5 (1982), 1109-1112.

Sindall,J.N. "The wave mode of walking locomotion." Journal of Terramechanics 1, 4 (1964), 54-73.

Smoliar, Stephen W.. A lexical analysis of labanotation. University of Pittsburgh Tech. Report, July 1978.

Snell,E.. Reciprocating Load Carrier. , 1947.

Speckert, G. "A computerized look at cat locomotion or one way to scan a cat." , A.I. Memo 374 (1976).

Steindler, A.. Mechanics of normal and pathological locomotion. Charles C. Thomas, Springfield, IL, 1935.

Stepaneko,Y. Dynamics of complex mechanisms. Mathematical Institute, . Belgrade

Stuart,D.G., Withey,T.P., Wetzel,M.C., Goslow,G.E.,Jr. Time constraints for inter-limb coordination in the cat during unrestrained locomotion. In control of posture and locomotion, New York, 1973, pp. 537-560.

Sun,S.S. A theoretical study of gaits for legged locomotion systems. Ph.D. Th., , 1974.

Sutherland, I.E.. A Walking Robot. The Marcian Chronicles, Inc., P.O. Box 10209, Pittsburgh, PA. 15232, 1983.

Sutherland, I.E. "Footprints in the Aspalt" International Journal of Robotics Research 3, 2 (1984). 
Taylor, C.R., Rowntree, V.J. "Running on two or four legs: Which consumes more energy?" Science 179 (1973), 179-187.

Taylor, C.R., Shkolnik, A., Dmpel, R., Baharav, D., Borut, A. "Running in cheetahs, gazelles, and goats: energy cost and limb configuration." Amer. J. of Physiology 227, 4 (1974), 848-850.

Tayior, C.R., Heglund N.C., McMahon, T.A., Looney, T.R. "Energetic cost of generating muscular force during running." J.Exp.Biol. 86 (1980), 9-18.

Terhune Jr., C.H., Fowler, D.R. Proposal for development of unmanned remotely-controlled vehicles to assist in mine rescue and recovery operations. Document 5020-84, US Bureau of Mines, Dept of Int., Jun, 1978. JPL Proposal 70-959

Tomovic,R. "On the synthesis of self-moving automata." Automation and Remote Control 26 (19-5).

Tomovic,R. "A general theoretical model of creeping displacement." Cybernetics IV (1961), 98-107. English translation

Tomovic,R., Bellman. "Systems approach to muscle control." Math. Biosci. 8 (1970).

Tomovic, R., Karplus,W.R.. Land locomotion -- simulation and control. , Opatija, Yogoslavia, 1961. Proc. Third International Analogue Computation Meeting

Tomovic,R., McGhee,R.B. "A finite state approach to the synthesis of bioengineering control systems." IEEE $L$ on Human Factors in Electronics 7, 2 (1966), 65-69.

Townsend,M., Seireg,A. "Optimal trajectories and control for systems of coupled rigid bodies." ASME Journal of Engineering for Industry (1972).

Trnkoczy,A., Bajd,T., Malezic,M. "A dynamic model of the ankle joint under functional electrical stimulation in free movement and isometric conditions." J. of Biomechanics 9 (1976), 509-519.

Urschel,W.E.. Walking Tractor. , 1949.

vanLeeuwen, J.L., Jayes, A.S., Alexander, R. McN. "Estimates of mechanical stresses in tortoise leg muscles during walking." J. Zool, Lond. 195 (1981), 53-69.

Vukobratovic,M. "Contributions to the study of anthropomorphic systems." Kibernetika 2(1972).

Vukobratovic,M. Dynamics and control of anthropomorphic active mechanisms. First Symposium on Theory and Practice of Robots and Manipulator Systems, IFTMoM, Udine, Italy, 1973, pp. 313-332.

Vukobratovic,M.. Legged locomotion robots and anthropomorphic systems. Research monograph, Institute Mihialo Pupin, Belgrade, Yugoslavia, 1975.

Vukobratovic,M. Legged locomotion robots: mathematical models, control algorithms and realizations. , 1975. Unpublished report

Vukobratovic,M., Frank,A.A. Legged locomotion studies. In Advances in External Control of Human Extremities, M.M. Gavrilovic, A.B. Wilson Jr., Belgrade, Eds., , 1969, pp. 407-430. 
Vukobratovic,M., Juricic',D. "Contribution to the synthesis of biped gait." IEEE $L$ on Biomedical Engineering BME-16(1969).

Vukobratovic,M., Okhotsimskii,D.E. "Control of legged locomotion robots." Proc. International Federation of Automatic Control Planary Session (1975).

Vukobratovic,M., Stepaneko,Y. "On the stability of anthropomorphic systems." Mathematical Biosciences 14 (1972), 1-38.

Vukobratovic,M., Stepaneko,Y. "Mathematical models of general anthropomorphic systems." Mathematical Biosciences 17 (1973), 191-242.

Vukobratovic,M., Frank,A.A., Juricic',D. "On the stability of biped locomotion." IEEE $t$. on Biomedical Engineering BME-17(1970).

Vukobratovic,M., Juricic',D., Frank,A.A. "On the control and stability of one class of biped locomotion systems." t. ASME Journal of Basic Engineering (1970), 328-332.

Vukobratovic,M., Ciric,V., Hristic,D., Stepanenko,J. Contribution to the study of anthropomorphic robots. , Paper 18.1, Proc. IFAC V World Congress, Paris, 1972.

Vukobratovic,M., et al. "Development of active exoskeletons." Medical and Biological Engineering (1973).

Vukobratovic,M., Hristic,D., Stojoljkovic,Z. "Development of active anthropomorphic exoskeletons." Medical and Biological Engineering (1974), 66-80.

Vukobratovic,M., Hristic,D., Stokic',D., Gluhajic',N. "New method of artificial motion synthesis and application to locomotion robots and manipulators." 0 .

Wetzel, M.C., Stuart, D.G. "Ensemble characteristics of cat locomotion and its neural control." Progress in Neurobiology 7 (1976), 1-98.

Wetzel,M.C., Atwater,A.E., Stuart,D.G. Movements of the hindlimb during locomotion of the cat. In Neural control of locomotion, R.N. Herman, S.Grillner, P.S. Stein, D.G. Stuart, Eds., Plenum Press, New York, 1975.

Williams,M., Lissner,H.R.. Biomechanics of human motion. W.B. Saunders Company, Philadelphia, 1962.

Wilson,D.M. "Insect walking." Annual Review of Entomology 11 (1966), 103-121.

Wilson,D.M. "Stepping patterns in tarantula spiders." J. Exp. Biol. 47 (1967), 133-151.

Winter,D.A., Robertson, D.G.E. "Joint torque and energy patterns in normal gait." Biological Cybernetics 29 (1978).

Winter,D.A., Greenlaw,R.K., Hobson,D.A. "Television-computer analysis of kinematics of human gait." Computer and Biomedical Research 5 (1972).

Wirta,R.W., Herman,R.. Some observations of relations among gait variables, 1975. Krusen Center for Research and Engineering Temple University, Moss Rehabilitation Hospital, Philadelphia 
Witt,D.C. A feasibility study on automatically-controlled powered lower-limb prosthesis. University of Oxford, . Department of Engineering Science Report

Wittenburg,J.. Dynamics of systems of rigid bodies. B. G. Teubner, 1977.

Yang,P. A study of electronically controlled orthotic knee joint systems. Ph.D. Th., The Ohio State University, Columbus, Ohio, 1976.

Zheng, Y. The study of a nine link biped model with two feet. Ph.D. Th., OSU, 1980. Report \#TH-80HH-058 
